\title{
JAMRS
}

OVERVIEW REPORT



NOVEMBER 2004

20004

Youth Poll Report and Crosstabulations
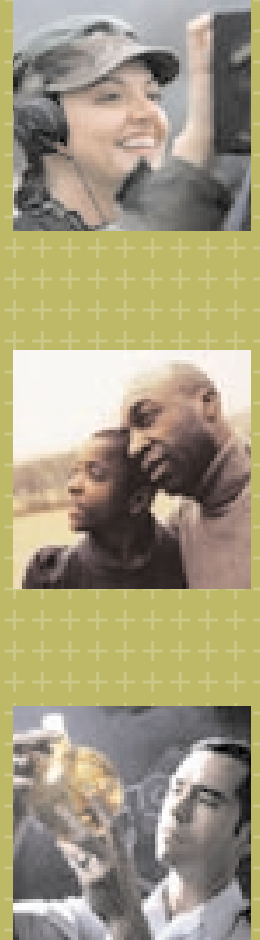


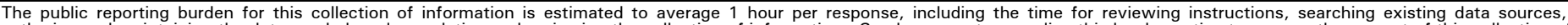

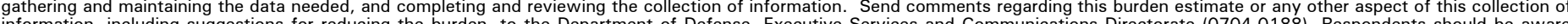

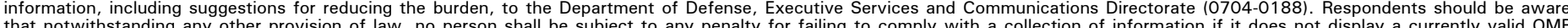

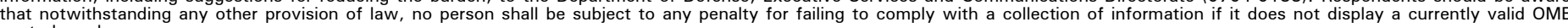
control number.

PLEASE DO NOT RETURN YOUR FORM TO THE ABOVE ORGANIZATION.
1. REPORT DATE (DD-MM- $Y Y Y Y)$ $\mathrm{XX}-07-2005$
2. REPORT TYPE
Final overview
3. DATES COVERED (From - To)

4. TITLE AND SUBTITLE

Youth Poll Report November 2004

DASW01-02-D-0002/0036

5b. GRANT NUMBER

5c. PROGRAM ELEMENT NUMBER

\section{6. $\operatorname{AUTHOR}(S)$}

Emanuel, S., Marsh, S. M., Marsh, K., Boehmer, M., Zucker, A.

\section{5d. PROJECT NUMBER}

5e. TASK NUMBER

5f. WORK UNIT NUMBER

\section{PERFORMING ORGANIZATION NAME(S) AND ADDRESS(ES)}

Joint Advertising, Research and Studies,

Defense Human Resources Activity,

4040 N. Fairfax Drive, Suite 200

Arlington, VA 22203-1613

9. SPONSORING/MONITORING AGENCY NAME(S) AND ADDRESS(ES)

Joint Advertising, Research and Studies,

Defense Human Resources Activity,

4040 N. Fairfax Drive, Suite 200

Arlington, VA 22203-1613

\section{DISTRIBUTION/AVAILABILITY STATEMENT}

Approved for public release; distribution is unlimited.

\section{SUPPLEMENTARY NOTES}

\section{ABSTRACT}

The Department of Defense (DoD) conducts Youth Polls on a regular basis to measure youth's perceptions of the military and propensity to enlist in the military. This report details the findings of the November 2004 Youth Poll.

The primary focus of the poll was to measure the likelihood of youth ages 16 - 21 to join the military and to identify the factors that influence their decision. In addition, this Youth Poll measured youth's favorability and knowledge of the military, their sources of impressions of the military, effective communications strategies, opinions of recruiters and perceptions of how supportive various people would be if they joined the military.

15. SUBJECT TERMS

Youth, Propensity

\begin{tabular}{|c|c|c|}
\hline \multicolumn{3}{|c|}{ 16. SECURITY CLASSIFICATION OF: } \\
\hline a. REPORT & b. ABSTRACT & c. THIS PAGE \\
U & U & U \\
\hline
\end{tabular}

17. LIMITATION OF
ABSTRACT
SAR

18. NUMBER

OF

PAGES

233
8. PERFORMING ORGANIZATION REPORT NUMBER

10. SPONSOR/MONITOR'S ACRONYMIS

11. SPONSOR/MONITOR'S REPORT

2005-001 


\section{PAGE LEFT BLANK INTENTIONALLY}




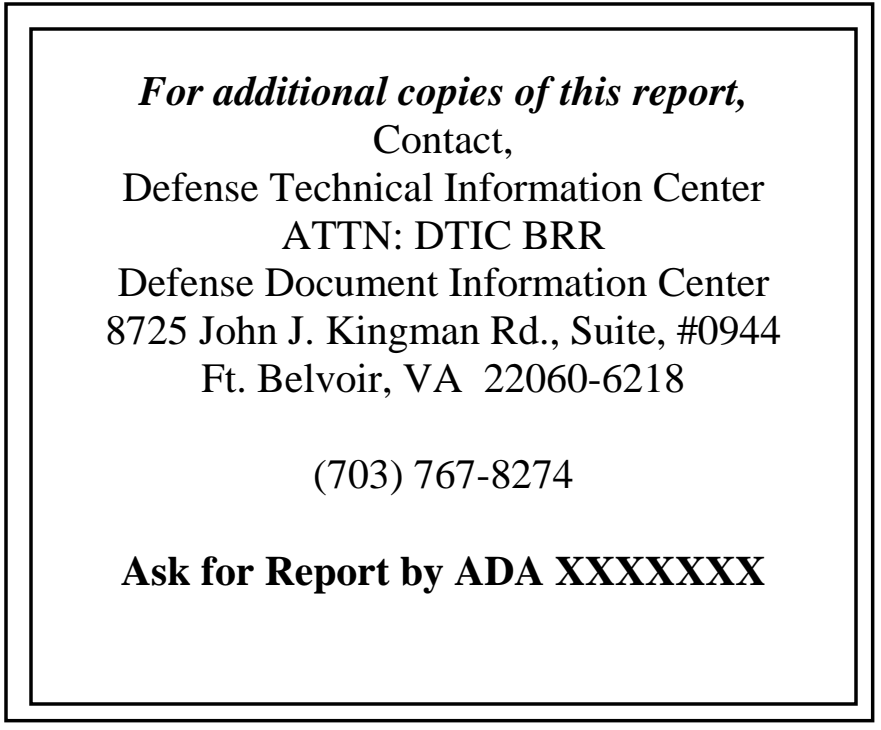




\section{PAGE LEFT BLANK INTENTIONALLY}




\section{DEPARTMENT OF DEFENSE YOUTH POLL WAVE 8 - NOVEMBER 2004}

\section{OVERVIEW REPORT}

Stacy Emanuel, Sean M. Marsh, Ph.D., and Kara Marsh, Ph.D.

Fors Marsh Group

Matt Boehmer and Andrea Zucker

Defense Human Resource Activity

Department of Defense

Defense Human Resources Activity

Joint Advertising, Market Research and Studies

4040 N. Fairfax Drive, Suite 200, Arlington, VA 22203-1613 


\section{ACKNOWLEDGEMENTS}

The work that was done to collect this information and produce this report was the result of a collaboration of efforts between DHRA, Fors Marsh Group, and Wirthlin Worldwide. The authors would like to thank the many youth who shared their time and opinions with us. 


\section{Executive Summary}

The Department of Defense (DoD) conducts Youth Polls twice a year to measure youth's perceptions of the military and propensity to enlist in the military. This report details the findings of the November 2004 Youth Poll.

The primary focus of the poll was to measure the likelihood of youth ages $16-21$ to join the military and to identify the sources of information that influence their decisions.

In addition, this Youth Poll measured youth's favorability toward and knowledge of the military, their sources of impressions of the military, effective communication strategies, opinions of recruiters, and perceptions of how supportive various people would be if they joined the military.

Overall, propensity has remained stable; minority propensity has dropped

Overall, youth's propensity to serve on active duty in each of the individual branches remained stable since last measured in May 2004. This is driven by White male propensity remaining stable. Black propensity, however, has dropped. This is most assuredly due to the declining view Blacks have of recent military actions. In addition to mounting attitudinal problems among Blacks, Hispanics are also starting to waver in their opinions of the military. This is already starting to affect propensity and should be monitored very closely.

Hispanic youth reported the highest level of propensity, but this number is down from the May 2004 poll. With the continual and dramatic decline in propensity among Blacks, Blacks now report the lowest propensity levels. Female propensity has also declined significantly since May 2004.
In addition, unemployed youth had higher propensity than youth who were employed.

Youth's consideration of the military has also dropped. Currently, 33\% of youth say they have never considered the possibility of joining the military. This number has significantly increased since May 2004.

Current events continue to take a toll on youth's attitudes toward the military, particularly for Black youth and females

In general, youth are not happy about where the country is heading, with $58 \%$ saying the country is heading in the wrong direction.

The majority of youth report that the War on Terrorism has negatively affected their likelihood of joining the military; Black and female youth reported being more negatively affected than any other group. In addition, Black youth were the least supportive of U.S. troops' presence in Iraq, the least likely to feel the war was justified, the most disapproving of the Bush Administration's handling of foreign affairs, and its use of U.S. Military forces.

Tracking evidence suggests these negative perceptions have been slowly emerging since the beginning of military operations in November 2001. Between November 2001 and November 2002 favorability toward the U.S. Military significantly dropped for Whites, Blacks, and Hispanics. Following the initial drop, White and Hispanic favorability stabilized at new, albeit lower levels. However, Black favorability continued to decline. To date, Black favorability has dropped almost twice as far since November 2001 as White or Hispanic favorability. 


\section{Executive Summary}

Youth economic outlook poor

Youth continue to report that they feel the labor market is relatively poor. The proportion of youth reporting that they expect the economy to be better in four years has been gradually declining since June 2003. Similarly, the proportion of youth reporting that it is impossible or very difficult for someone their age to find a fulltime job in their community has been steadily increasing since November 2001. Lastly, perceptions of military job pay relative to civilian pay have significantly increased since May 2004, despite the fact that the military has recently been providing larger monetary incentives for enlistment.

\section{Recruiters}

Only $59 \%$ of youth report they have spoken with a military recruiter, unchanged from two years ago. However, youth's trust in recruiters has changed. Youth today are less likely to believe people get the benefits promised to them by recruiters, less likely to believe that recruiters are providing trustworthy information, and less likely to believe that recruiters are painting a truthful picture of the military, from just 2 years ago.

Focusing efforts on direct mail activties may be a very effective use of recruiters dollars for non-propensed minority groups Focusing efforts on direct mail activities may be a more effective use of recruiting dollars for certain youth markets than recruiter phone calls. Minority youth, particularly non-propensed youth, report they are more likely to read mail from recruiters than to talk to them on the phone. These groups also report being more likely to discuss with parents information they receive in the mail than information they receive from a phone call. It is important to note however, that $44 \%$ of youth do say they are likely to speak with a recruiter when one calls. A two-tiered approach where recruiters attempt to get a "foot in the door" with a direct-mail piece and then follow up with a phone call may be extremely effective in maximizing recruiting resources in certain areas.

More conversations between youth and influencers about joining the military are necessary

Youth actively seek advice from their parents about their future, however, few of these conversations include the military. These conversations are just as likely to be positive as they are negative. Youth are also seeking advice from educators regarding their future, albeit at a low rate. When the conversation with educators does include the military, the response from educators is more likely to be positive than negative. Youth report that recruiting efforts help to promote these types of communications. Approximately $30 \%$ of youth report that if a recruiter called their home or if they received recruiting materials in the mail, they would talk to their parents about it. In addition, those who speak with influencers are more propensed for service, more favorable toward the military, and more knowledgeable.

The sources that youth are most likely to use are the sources they trust most When looking for information regarding the military, youth are most likely to go to the Internet. They report using the Internet in general as well as military Web sites. After the Internet, youth report tapping a number of different people including family, friends, educators, and military recruiters, as well as mail for information about the military. 


\section{Table of Contents}

Section 1. Introduction

Background and Purpose....................................................................................1-1

Approach .................................................................................................................1-2

Methodology ......................................................................................................1-4

Respondent Profile.....................................................................................................1-5

Section 2. Future Plans and Propensity

Future Plans and Propensity ..........................................................................2-1

Future Plans: Overview ...........................................................................2-2

Future Plans: Education .................................................................................2-3

Future Plans: Employment ..........................................................................2-5

Future Plans: Military ...................................................................................2-8

Summary .......................................................................................................... 2-10

Section 3. Attitudes Towards and Communication Strategy Regarding the

Military

Attitudes Towards and Communication Strategy Regarding

the Military...................................................................................................................3-1

Favorability ............................................................................................3-2

Knowledge ……................................................................................................................

Current Events.......................................................................................................3-4

Communication Strategy and Recruiters.........................................................3-5

Military Advice ........................................................................................................

Sources of Impressions ..................................................................................... 3-8

Summary .....................................................................................................................

Section 4. Service and Component Propensity

Unaided Propensity ................................................................................................4-1

Aided Propensity: Active Duty ............................................................................4-3

Aided Propensity: Reserve and National Guard ............................................4-5

U.S. Army Favorability and Propensity ..........................................................4-6

U.S. Navy Favorability and Propensity ............................................................4-7

U.S. Marine Corps Favorability and Propensity ..........................................4-8

U.S. Air Force Favorability and Propensity....................................................4-9

U.S. Coast Guard Favorability and Propensity ..............................................4-10

U.S. Reserves Favorability and Propensity ...................................................4-11

U.S. National Guard Favorability and Propensity .......................................4-12

Summary ...................................................................................................................4-13 


\section{Table of Contents}

(continued)

\section{Section 5. Summary and Conclusions}

Summary and Conclusions ......................................................................................5-1

\section{Appendices}

Appendix A: Detailed Crosstabs ........................................................................A-1

Appendix B: Technical Report........................................................................B-1

Appendix C: Final Questionnaire ............................................................. 
OVERVIEW REPORT

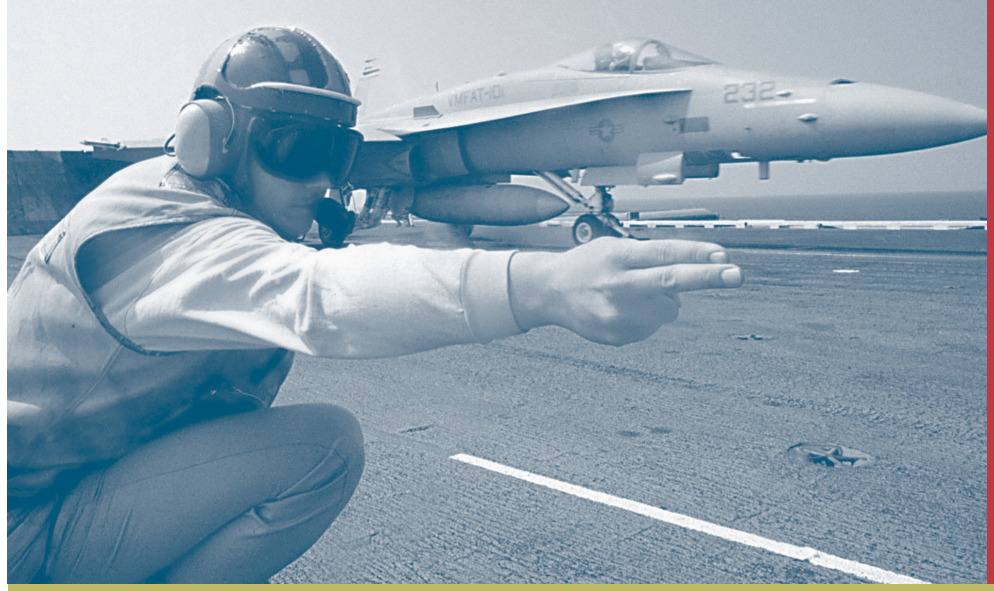

The primary goal of the youth poll is to provide regular tracking of propensity the likelihood that youth will join the military. Section 0 ne covers the approach and methodology used in the N ovember 2004 poll to track propensity.

\section{Section One}




\section{PAGE LEFT BLANK INTENTIONALLY}


Recruiting goals are determined in part by anticipated shortfalls in the specified size and skill distribution needed by the U.S. Armed Forces. The causes of these shortfalls include attrition during the first term of service, voluntary losses based on those who do not re-enlist, or an overall increase in the number of personnel required.

However, the Department of Defense (DoD) faces a multitude of challenges in recruiting the required number of qualified personnel. American youth's disposition for military service has been gradually declining since the 1980's. This decline, coupled with factors such as eligibility and increasingly attractive alternative options available to youth, has caused some concern among the military recruiting community regarding future recruiting goals.

The difficulty of recruitment threatens the vitality and quality of the U.S. Military if left unchecked. The Youth Poll aims to provide needed intelligence regarding attitudes toward the military, perceptions of the military, and propensity toward joining the military, to guide marketing, advertising, and recruiting efforts to maintain the quality and required size of the all-volunteer force.

The central concept measured by the Youth Polls is called propensity, which is the stated likelihood that a youth will enlist in the military or one of the military Services in the next few years. The concern about falling propensity and the desire to understand the phenomenon more fully were the impetus for the start of Youth Attitudinal Tracking Study (YATS) in 1975 and then the Youth Polls in 2001.
Each Youth Poll measures military propensity as well as youth's favorability toward the military, perceived knowledge of the military, perceptions of current economic conditions, and reactions to current events. In addition, each Youth Poll focuses on one of three special topics found to directly affect military recruiting. These topics include:

1. Factors that affect propensity, including youth's attitudes and views on the military, the influence adults have on youth's attitudes, youth's perceptions of current events, and their confidence in successfully performing military-related duties.

2. Youth's ability to meet the physical, medical, moral, and other standards for enlistment set by the U.S. Military.

3. The source of youth's military impressions and the influence that these sources have on propensity and consideration of military service.

The November 2004 Youth Poll focuses on the third topic detailed above: the sources of youth's military impressions and the influence these sources have on propensity.

\section{Predicting Future Plans}

The purpose of this report is to examine options available to youth following high school graduation, and to present information on the sources and influences of youth's military impressions. The focus of this report is not on answering the question of what has caused the military recruiting shortfalls of recent years, but rather on identifying factors likely to influence future recruiting effectiveness. 


\section{Approach}

Researchers have developed theories of behavioral prediction that can be applied in various situations, not only to predict whether or not people will engage in certain behaviors, but also to better understand what drives behavior and how those drivers can be manipulated or influenced.

The Theory of Reasoned Action is a leading explanatory model of behavior and is used by the Joint Advertising, Market Research and Studies (JAMRS) program to help guide some of its survey efforts. According to this model, behavior is primarily driven by intention (propensity in the case of military enlistment).

To predict propensity, one must identify certain attributes that differentiate youth who are propensed from those who are not. A growing body of evidence suggests that propensity can be explained, in large part, by knowing something about a person's attitudes, subjective norms, and selfefficacy.

Attitudes are a function of one's beliefs that performing a behavior will lead to certain outcomes and the importance of those outcomes. A subjective norm is the perception that the important others in one's life will think that one should or should not perform the behavior in question. Confidence in performing the behavior is based on years of research on self-efficacy by Albert Bandura and his colleagues.

The model at the bottom of this page shows that military enlistment, like any behavior, is most likely to occur if one has a strong intention to perform that behavior, if one has the necessary skills and abilities (i.e., meets military enlistment standards), and if there are no environmental constraints preventing the behavior (e.g., subjective norms).

Use of a model-based approach such as this provides several advantages. Principal among these is use of the findings to determine strategic direction. For example, very different interventions would be necessary if one has formed an intention but is unable to act, than if one has little or no intention to perform the behavior or if one is not engaging because of social pressure from important people in one's life. A model-based approach that integrates these multiple components aids decision-making by providing a more comprehensive and integrative platform of information from which to make decisions.

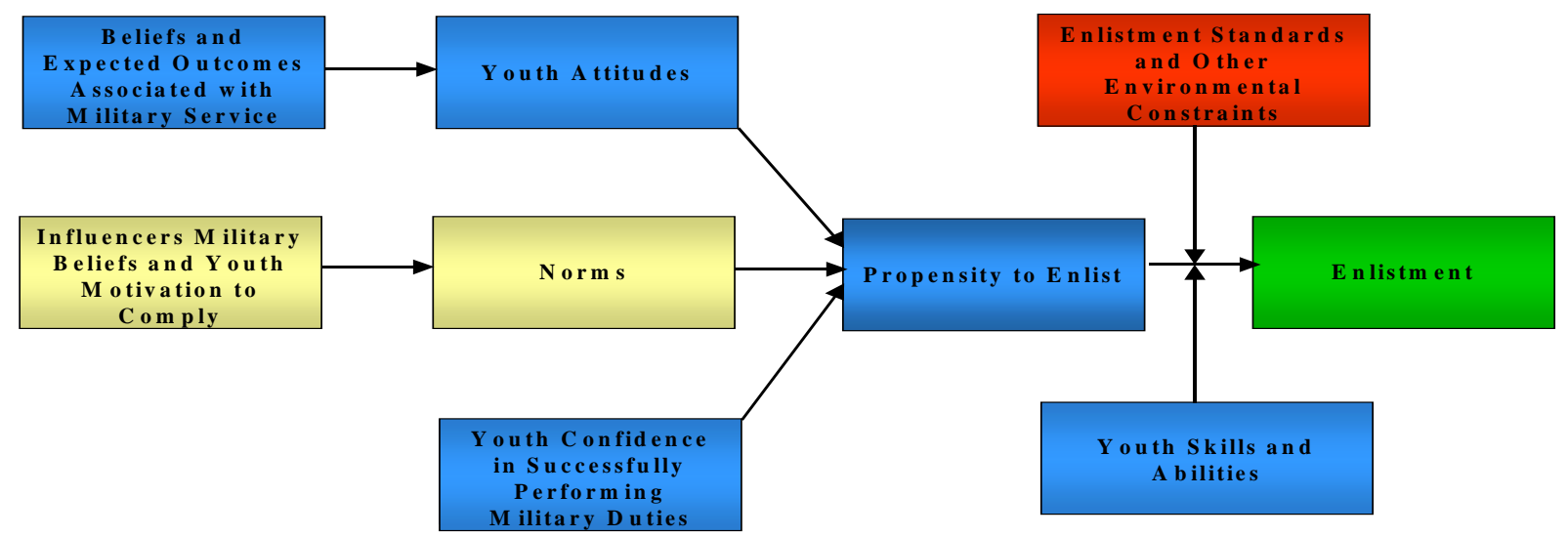




\section{Approach}

(continued)

Researches have found that where youth get the information they use to make decisions has a great influence on their attitudes and perceived importance of outcomes. In addition, understanding where youth typically get their impressions can aid the military in their own marketing and advertising efforts. Thus, the November 2004 Youth Poll examines where youth get their information and their perceived trust in this information.

This report is structured around the conceptual model previously mentioned, but specifically addresses youth's sources of military impressions and propensity to join the military. Following this introduction, the report begins in Section 2, which details findings regarding the U.S. Military including favorability toward the military, propensity of youth to join the military, knowledge about the military, and determinants of joining the military. This section also includes information on current events and economic indicators.
Section 3 provides information on youth's sources of impressions of the military and the trust they have in each source. Youth's opinions of recruiters as well as their preferred method of communication with recruiters are also examined. Finally, Section 4 details the same favorability, propensity, knowledge, and determinants of propensity information as Section 2, but provides the information for each of the individual Services and components.

Section 5 presents the conclusions and recommendations based upon analyses provided in each of the chapters.

\section{Naming Convention}

Throughout this report, we refer to three race/ethnic groups: Whites, Blacks, and Hispanics. These names correspond to the group names used by the Census Bureau. 


\section{Methodology}

The November 2004 Youth Poll used random digit dialing administered via computer-assisted telephone interviews. Data were collected between October 7 , 2004, and November 30, 2004.

American households were screened for the target audience: Americans between the ages of 16 and 21 who have never served in the U.S. Armed Services and are not enrolled in a postsecondary Reserve Officers' Training Corps program. In the case that more than one person in the household met these criteria, the respondent with the most recent birthday prior to the interview date was selected.

The sample size of the November 2004 Youth Poll was 3,345 completed interviews. In this design, telephone households were sampled with stratified random sampling within one of two stratums in the first stage. In the second stage, one eligible person was randomly sampled within the household. The two strata used in this design were defined as a "low density" stratum, which had a concentration of less than $30 \%$ Blacks in the calling prefix, and a "high density" stratum, with a concentration of more than $30 \%$ Blacks.

On average the survey took 20 minutes to complete. The data were weighted by gender, age, race/ethnicity, and education to reflect the general population based on Current Population Survey data from the U.S. Census.

To find confidence intervals and test hypotheses using this data, the variance for the estimated statistics that take into account the properties of the study design must be calculated. The authors conducted these analyses using Taylor-series linearization. Appendix B contains a detailed technical assessment and description of the research methodology and variance estimation procedures. 


\section{Respondent Profile}

The November 2004 Poll was conducted via telephone using random digit dialing. The following charts display the demographic segments of the 3,345 survey respondents:

Age

$>$ Gender

$>$ Race/ethnicity

$>$ Education/school (currently and completed)

$>$ Grades

$>$ Employment status

$>$ Hours worked per week

$>$ Family information

$>$ Geographic area

Age

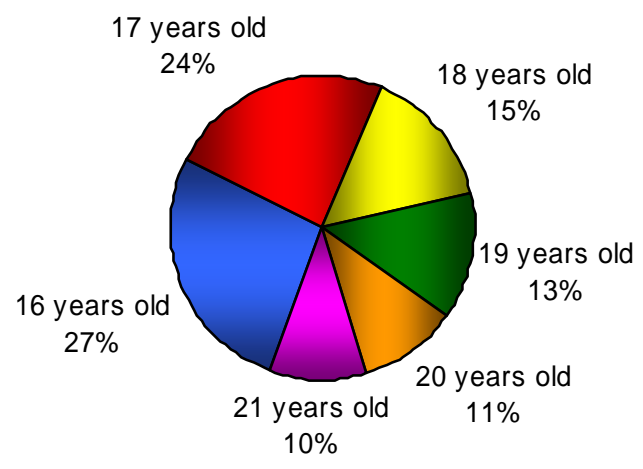

Gender

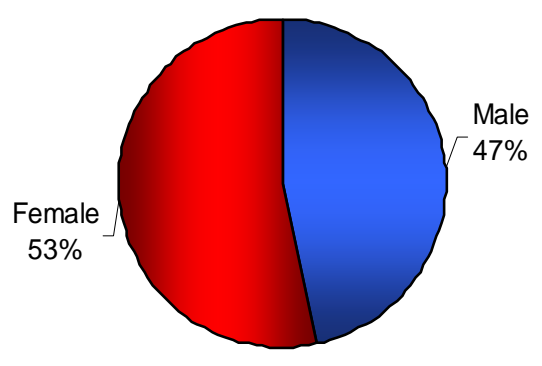

Which of the following best describes your race?



Page 1-5 Department of Defense November 2004 Youth Poll 


\section{Respondent Profile}

(continued)

Highlest Level of School Completed

Current Education Levels

(those currently enrolled)

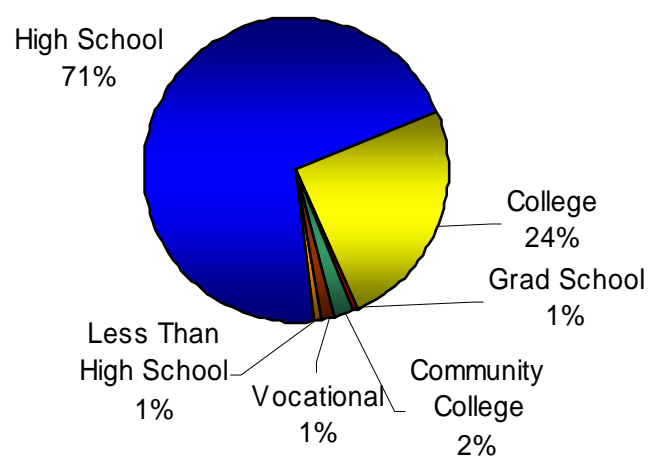

(those not currently enrolled)

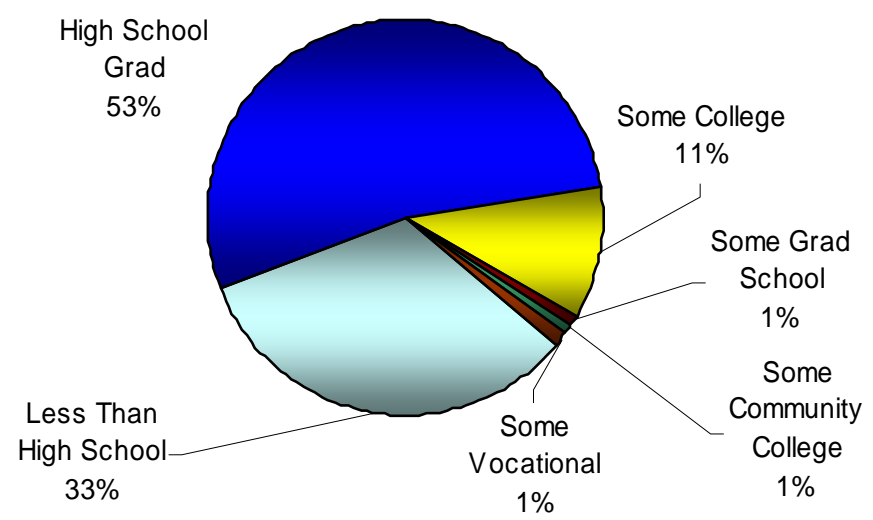

What grades do you or did you usually get in high

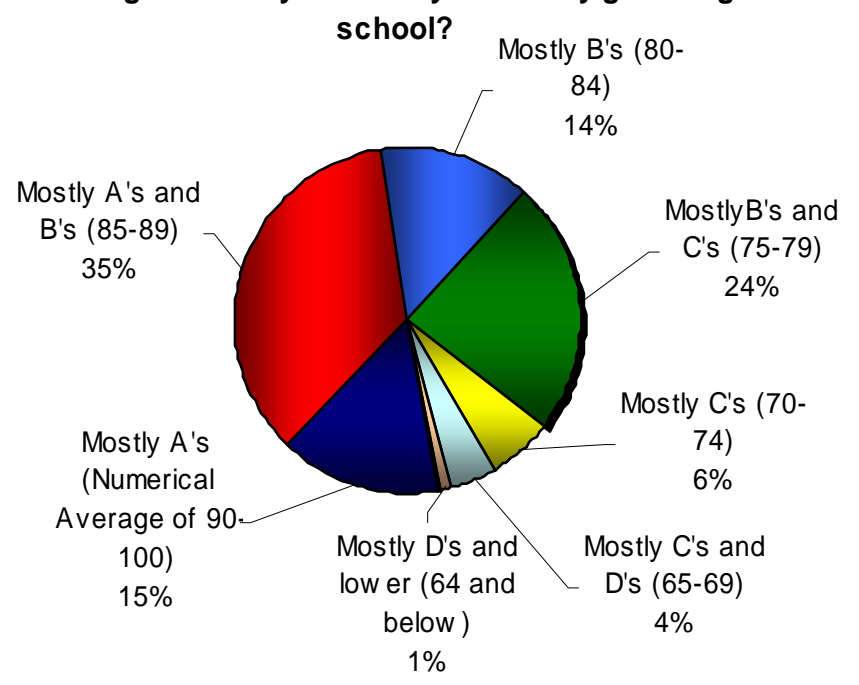




\section{Respondent Profile}

(continued)

Are you currently employed either full- or part- time?

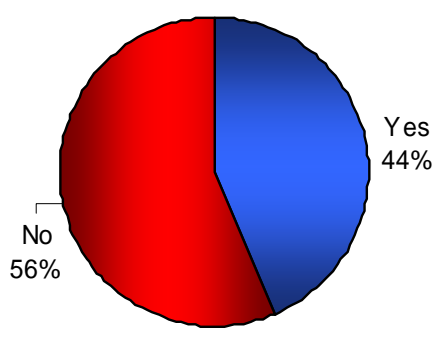

How many hours per week do you work at your job?



Has your [fam ily m e m ber] ever served in the U.S. m ilitary?


Page 1-7 Department of Defense November 2004 Youth Poll 


\section{PAGE LEFT BLANK INTENTIONALLY}


OVERVIEW REPORT

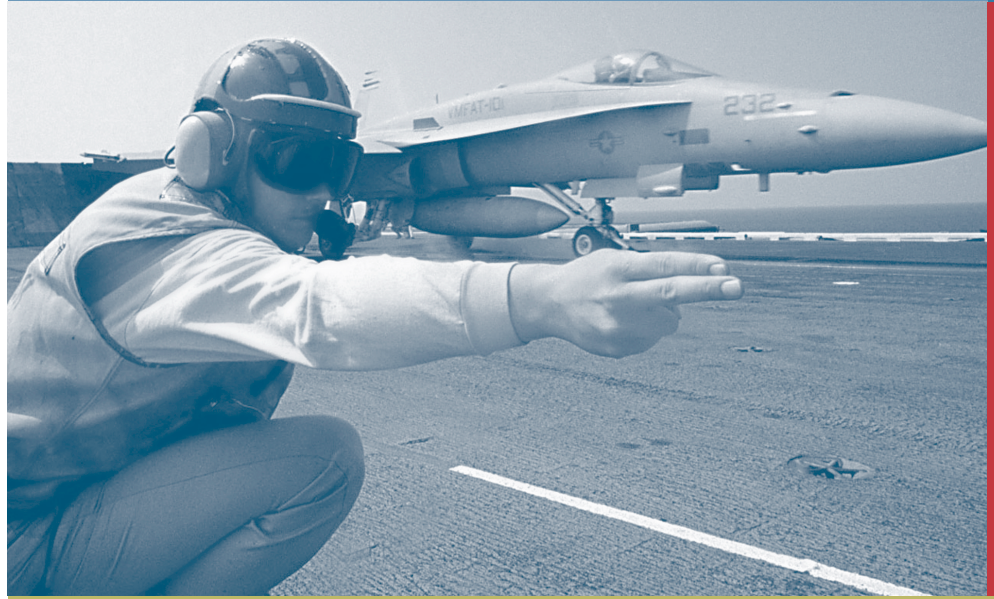

The focus of Section Two is on youth's plans for the future, including how the military may or may not fit into those plans.

Section Two 


\section{PAGE LEFT BLANK INTENTIONALLY}




\section{Future Plans and Propensity}

The previous section covered the background and purpose of the Youth Polls. This section explores young people's plans for the future and how the military may or may not fit into those plans.

Because the main goal of the Youth Poll is to answer the research question, "What is the propensity of American youth to enlist in the military?" this section will provide an overview of youth's plans for the future, based on findings from the November 2004 Youth Poll.

Two primary outcome measures are created from the Youth Poll propensity questions: Composite Active Duty Propensity (measuring youth's likelihood to enlist for active duty) and Composite Reserve Propensity (measuring the likelihood of youth to enlist in the National Guard or the
Reserves). In addition to the propensity questions referring to youth's specific plans to join the military, several questions were asked regarding their future plans for both education and work.

This section will address the questions asked to youth regarding their future plans and will provide a summary of the findings. Specific questions regarding individual Services can be found in Section 4 of this report. 


\section{Future Plans: Overview}

To determine youth's interest in military service, the Youth Poll asks several questions about youth's future plans, including their plans for education, work, and the military. It also specifically asks them about their intentions to join the military.

\section{Future Plans}

Youth were first asked what their plans were for the near future. Not surprisingly, education is the focus for the majority of youth.

Overall, $58 \%$ of youth indicated that they would go to school, with almost half of these youth (44\%) mentioning that they would attend school full time.
Fifty-one percent reported that they intend to work either full or part time. This percentage has dropped slightly, but the drop is not significant. It is important to note, however, that many of the youth who report working also report that they intend to go to school. For many of these youth, it may be reasonable to assume that working is not their primary goal, but a way to help subsidize schooling. However, a little more than a third of youth (34\%) do indicate that they will work full time.

Finally, $4 \%$ of youth reported that they intend to join the military. This proportion has remained relatively stable since the beginning of the Youth Polls in April 2001.

What do you think you might be doing "Once you finish high school"lonce you finish college"l"in the next few years"?

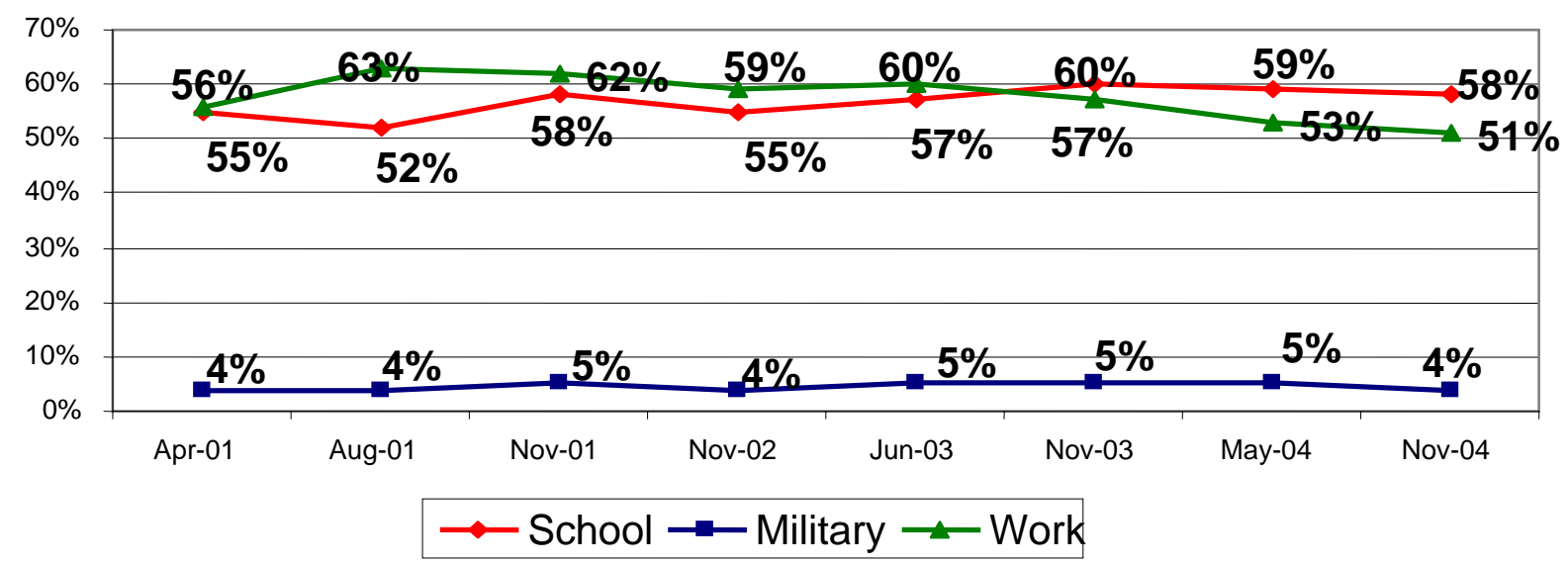




\section{Future Plans: Education}

In examining youth's responses to the openended question regarding their future plans, some very important demographic differences appeared. While $64 \%$ of females reported immediate plans for further education, only about half of males (52\%) mentioned further education. In fact, male youth were just as likely to mention going to work $(52 \%)$ as they were enrolling in school. Female youth, in contrast, were significantly more likely to mention school than work plans (49\%).

Interestingly, Whites were less likely to mention school $(55 \%)$ than were Blacks $(62 \%)$ or Hispanics (62\%). This overall statistic, however, may be somewhat misleading. Whites are equally likely at younger ages (16-18) to mention school. In fact, at 16 and 17 years old, approximately $80 \%$ of all youth report planning on attending school in the near future. However, after that point, Whites' likelihood of mentioning school drops at a faster rate than that of Blacks or Hispanics.

One possible explanation for this is the age at which Whites begin college. Although equal proportions of Whites and Blacks intend to pursue higher education, Whites in fact begin pursing that goal at an earlier age. When asked about future plans, more Whites may be thinking ahead to the jobs they will be working in once they finish college. In contrast, a larger proportion of Blacks and Hispanics may still be taking the steps necessary to begin their higher education.

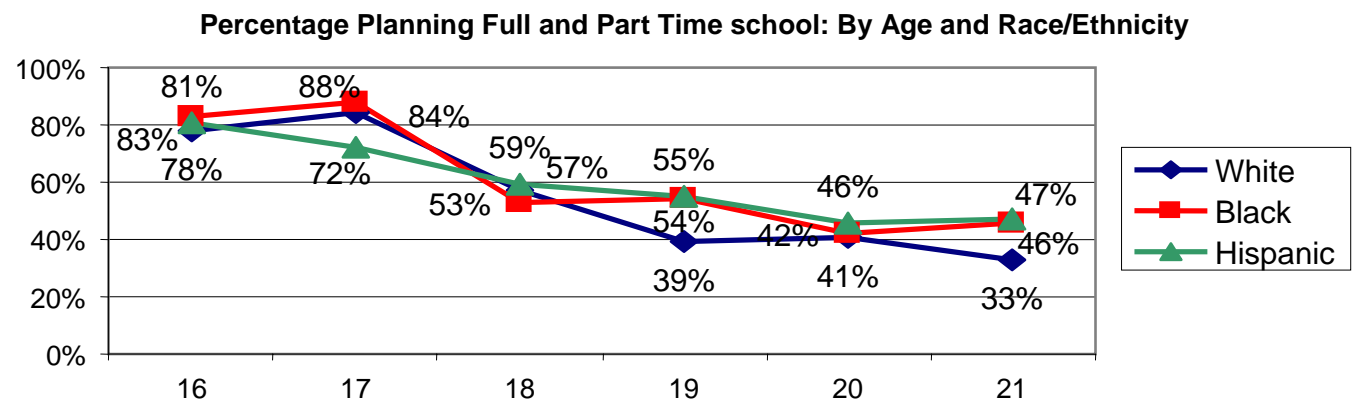

Percent with Some College: By Age and Race/Ethnicity






\section{Future Plans: Education}

(continued)

\section{Type of Higher Education}

In total, 58\% of youth reported plans to attend school (full time or part time). On follow-up, the majority of these youth (59\%) indicated specific interest in attending a 4-year college or university. However, Hispanics tended to be less interested in 4-year colleges and more interested in 2-year colleges, or vocational schools.

What kind of school you would like to attend? (subset)

\begin{tabular}{lc|c|c|c|c} 
& Total & White & Black & Hispanic \\
\cline { 1 - 2 } & $59 \%$ & $60 \%$ & $61 \%$ & $51 \%$ \\
2-year college or university & $18 \%$ & $16 \%$ & $20 \%$ & $23 \%$ \\
Vocational, business or trade school & $11 \%$ & $11 \%$ & $11 \%$ & $13 \%$ \\
Graduate or professional school & $10 \%$ & $11 \%$ & $6 \%$ & $11 \%$
\end{tabular}

These racial/ethnic differences represent the culmination of differences at various progression points in the education pipeline (U.S. Department of Education 2002). For example, students from different racial/ethnic backgrounds have different likelihoods of graduating from high school and attending college, with Whites having a higher rate of educational progress over their Black and Hispanic counterparts.

Looking at data from the National Education Longitudinal Study of 1988, there are three milestones that are traditional indicators of student progress. These are on-time attainment of a high school diploma, enrollment in a postsecondary institution within the year following high school graduation, and attainment of a postsecondary credential within the "scheduled" time frame. This study found that racial/ethnic differences emerged at the first milestone, the receipt of a regular ontime high school diploma.
White students were more likely than Black and Hispanic students to receive a regular on-time diploma, with $82 \%$ of White students doing so compared with $72 \%$ of Black students and $67 \%$ of Hispanic students. When these on-time high school graduates reached the next milestone, immediate entry to a postsecondary institution, similar patterns emerged. White students were more likely than their Black counterparts to enter postsecondary institutions, but no differences were detected in the rates of immediate enrollment between White and Hispanic students.

Finally at the third milestone, Black and Hispanic students who had graduated on time and immediately enrolled in a postsecondary institution were again found to have lower attainment rates than their White peers, indicating that racial/ethnic educational differences have been found by many researchers to start early on. 


\section{Future Plans: Employment}

In total, $51 \%$ of youth reported plans to enter the work force in the near future. Examined by race/ethnicity, Whites $(55 \%)$ were significantly more likely to report plans to work than were Blacks (42\%) or Hispanics (41\%).

Of the youth who were considering working, more than half (59\%) reported they planned to work at a job that could begin a long-term career. ${ }^{\mathrm{i}}$ The remainder reported they were interested in either temporary employment while they finished school or in any job that provides a living wage.

Career

Type of Work Interested In (subset)

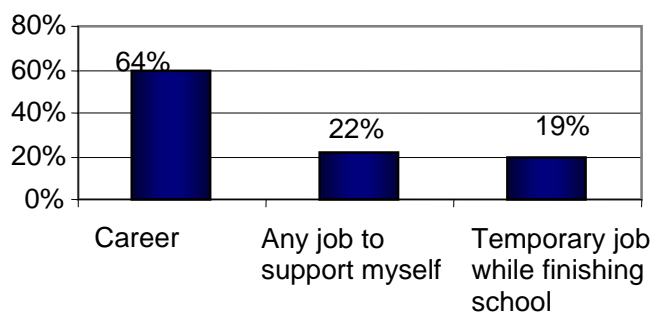

The size of the youth population interested in pursuing a career in the immediate future represents an important subpopulation for military recruiting. This is particularly true for those youth who have decided to enter the work force rather than pursue higher education. For these youth, enlisting in the military is an option that may be comparable on many dimensions while also offering unique benefits not provided by other career options.
Almost 9 in 10 young adults agreed

90\% "college is not for everyone," and more than 8 in 10 said many people do succeed without a college education.

Source: Johnson, Duffet \& Ott. (2005). Life After High School: Young People Talk About Their Hopes and Prospects. Public Agenda

Not surprisingly, older youth and youth already in college are most interested in pursuing a career in the near future.

There were also differences by race/ethnicity in regard to pursuing a career. Overall, Hispanics (24\%) were less likely to report they intended to pursue a career in the near future than were Whites (36\%). Blacks were between Whites and Hispanics at $26 \%$. As already noted, these findings may highlight nothing more than the speed at which the different race/ethnicities progress through school.

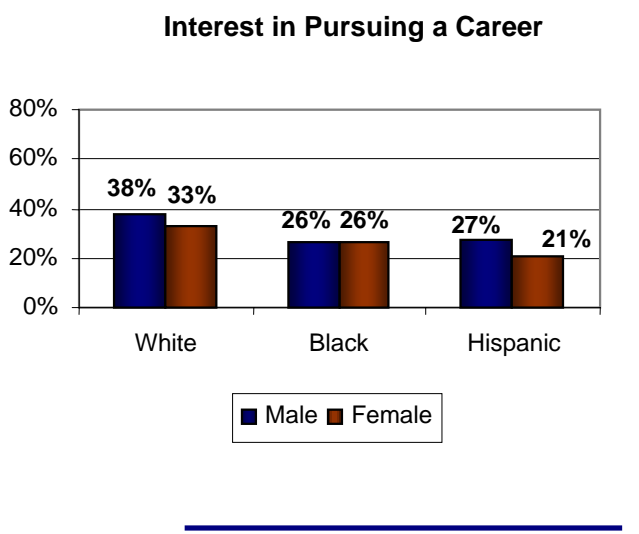

\section{Did You KNOW?}

In $2003,13 \%$ of youth ages $16-24$ were neither enrolled in school nor working, a decrease from 16\% in 1986.

(Source: Condition of Education 2003) 


\section{Future Plans: Employment}

(continued)

\section{Employment Difficulty}

Another factor that may influence employment intentions is perceived difficulty of finding a job. Overall, $32 \%$ of youth said it would be very difficult or almost impossible to obtain full time employment in their community.

Since November 2001, there has been a growing gap between perceptions of employment difficulty among Blacks and other racial groups. In the latest poll, that gap has grown to $13 \%$ as shown in the chart below. Although Blacks' attitudes toward the military appear to be worsening (as will be discussed later in this report), these perceptions may be one reason why recruitment propensity levels among Blacks are remaining somewhat stable.

\section{Employment Difficulty: By Race/Ethnicity}

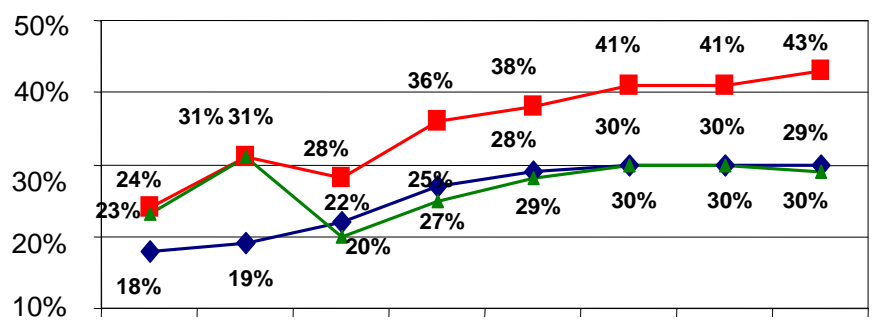

Apr-01 Aug-01 Nov-01 Nov-02 Jun-03 Nov-03 May-04 Nov-04

$\neg$ White $\_$Black $\multimap$ Hispanic

\section{Current Employment}

Employment disparity is prevalent in current employment status, as well. Whites (56\%) are more likely to be working than Hispanics $(43 \%)$ or Blacks $(41 \%)$. This difference holds across all ages, but the most substantial differences appear to be between youth ages $19-21$.

This same trend is found in youth as young

Employment Status: By Race/Ethnicity and Age

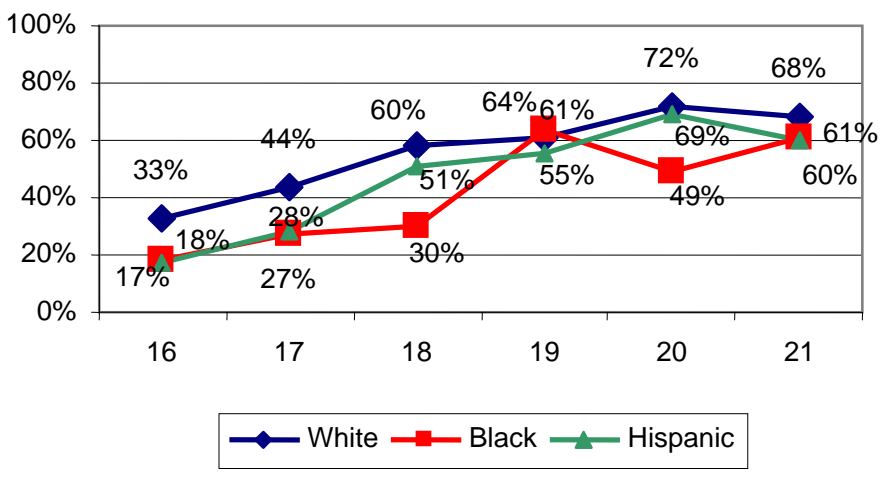

as ages 13 and 14. Entry into the labor market often begins early, with about half of youth ages 12 and 13 reporting that they work (Rothstein \& Herz, 2000). But as with older youth and adults, employment rates continue to be higher for Whites ages 13 and 14 than for Blacks or Hispanics ages 13 and 14 (National Longitudinal Transition Study 2). Moreover, when employed, Black youth are more likely to earn lower wages than are White youth.

\section{Did You KNOW?}

Unemployment among youth ages 16-19 is substantively higher for Blacks $(28.9 \%)$ than it is for either Whites $(15.3 \%)$ or Hispanics $(19.1 \%)$.

(Source: U.S. Bureau of Labor Statistics 2004) 


\section{Future Plans: Employment}

(continued)

\section{Job Pay}

The November 2004 Youth Poll asked youth if they were more likely to have a good paying job in the military or a civilian job. Fifty-nine percent felt that they would be just as likely to have a good paying job in the military as they would in the civilian sector. Twenty-percent felt that they would be more likely to have a good paying job in the military, and $20 \%$ felt that they would be more likely to have a good paying civilian job. Blacks and propensed youth are most likely to believe they have a better chance of having a good paying job in the military.

Military Pays Better

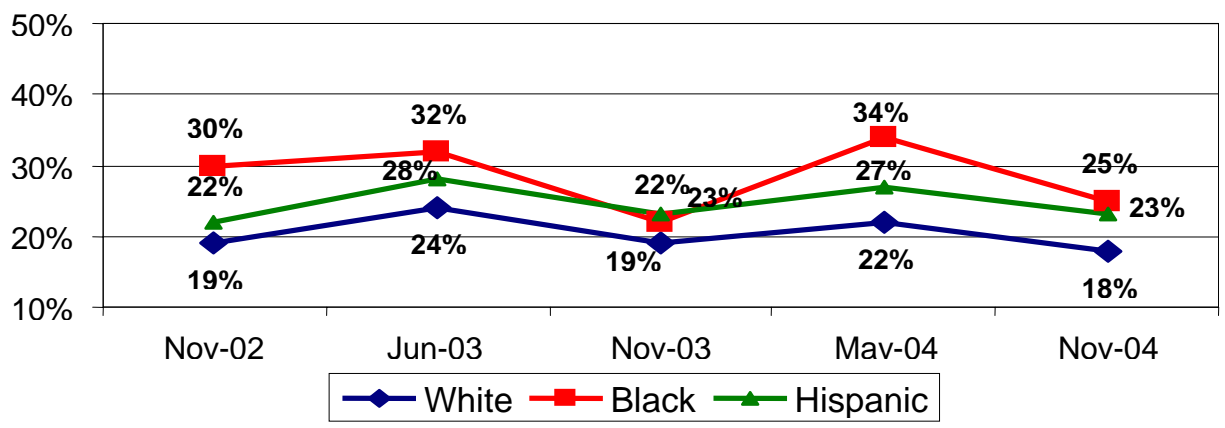

Economic Outlook

When asked about the future of the economy, youth were divided. Thirty-five percent of them felt that the economy will be better four years from now, compared with $38 \%$ in May 2004. Additionally, 35\% thought the economy will remain the same, while $29 \%$ thought the economy will only get worse. When observed across race/ethnicity, White and other youth were

\footnotetext{
According to the November

$58 \% \quad 2004$ Youth Poll, the majority of youth believe that things in this country have gotten pretty seriously off track.
}

the most optimistic. This could be the result of current unemployment trends, as only $15 \%$ of White youth are unemployed, while $29 \%$ of Black youth and $19 \%$ of Hispanic youth are unemployed. This trend has remained relatively stable since 1994, except for a small dip in 2000.






\section{Future Plans: Military}

\section{Consideration}

The Youth Polls ask whether or not youth have ever considered the possibility of military service. Military consideration represents a key aspect of the enlistment decision and a possible precursor to serious military intent.

The number of youth that have considered joining the military has decreased since the last Youth Poll. Currently, only 20\% of youth report giving the military serious consideration, while $47 \%$ give joining the military some consideration and $33 \%$ report having never considered the military as an option after high school.

What do you think you might be doing "once you finish high school"/once you finish college" $/$ "in the next few years"?

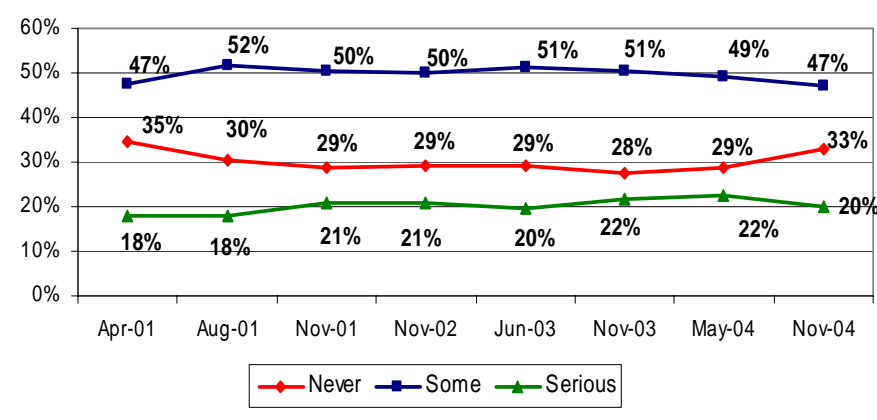

By race/ethnicity, Blacks (41\%) were significantly more likely to report having never considered military service than were Whites (30\%) or Hispanics (36\%); unfortunately, these percentages have all increased since the last Youth Poll.
Proportion of Hispanics who $36 \%$ report never having considered military service, up from $26 \%$ in May 2004.

After consideration, military propensity, or intention to join the military, is the second key indicator of joining the military. Propensity is measured by directly asking youth how likely (definitely, probably, probably not, definitely not) they are to join the military. Youth who indicated definitely or probably are considered propensed.

Overall, $22 \%$ (5\% definitely, $17 \%$ probably) of male respondents said it was likely they would serve. Female respondents were less propensed, with only $7 \%$ saying it was likely that they would serve (1\% definitely, $6 \%$ probably).

How likely is it that you will be serving in the military in the next few years?

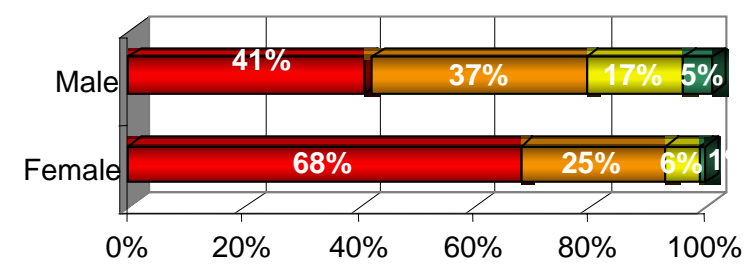

Definitely Not $\square$ Probably Not $\square$ Probably $\square$ Definitely 


\section{Future Plans: Military}

(continued)

Male propensity did spike immediately following the events of $9 / 11$, and although it has fluctuated somewhat, propensity is still slightly elevated above levels prior to 9/11.

Youth Military Propensity: By Gender

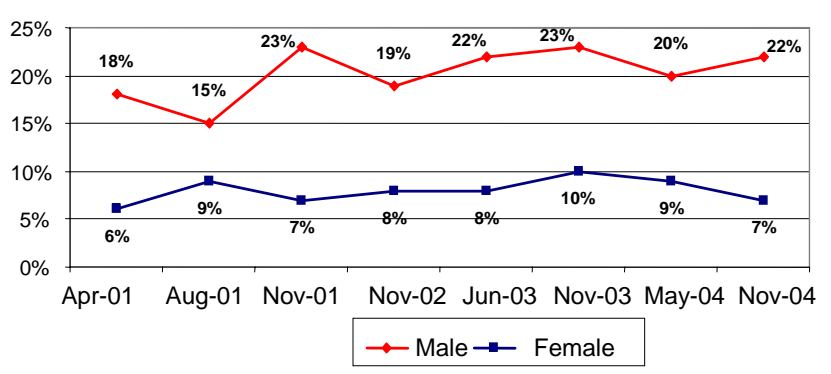

Broken out by race/ethnicity, Hispanic males reported the highest level of military propensity, with $28 \%$ responding that they would probably or definitely serve in the military in the next few years.

Although Hispanic males report the highest level of military propensity, this estimate is slightly down since the last Youth Poll in May 2004. Like their male counterparts, Hispanic females report the highest level of military propensity. Additionally, female Hispanics and Blacks are significantly more likely to be propensed than are female Whites.

Military Propensity: By Race/Ethnicity and Gender

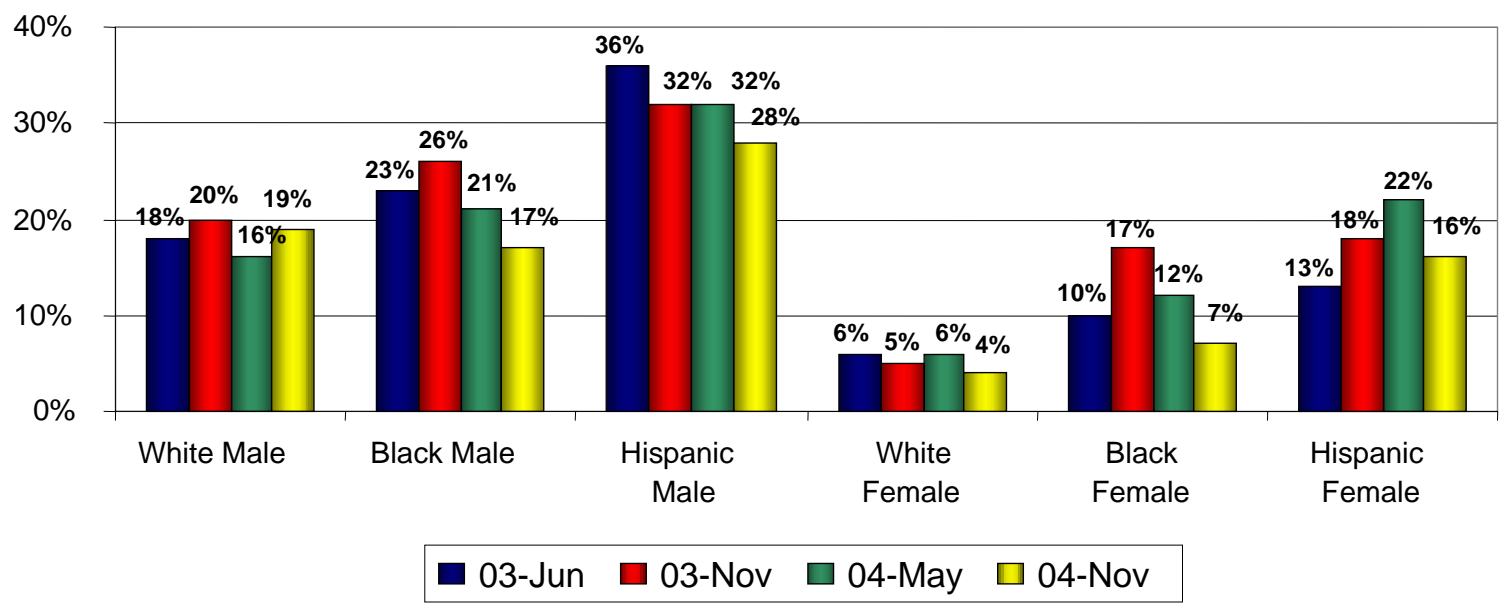




\section{Summary}

Section 2 focused on youth's future plans and propensity by examining their views of the military as well as their views on education and potential career options. Future career options were examined across race/ethnicity as well as gender.

\section{Future Plans}

Currently, American youth are focused on education and work, with military enlistment an afterthought, at best. The number of young people interested in post secondary education is on the rise, so continuing to focus on the educational benefits of the military (i.e. highlighting that one can serve in the Reserves and go to college at the same time) would seem very beneficial. In addition, most youth (64\%) who are looking for a job are still looking for one that could become a long-term career. Pointing out the long-term careers that can be established while serving in the military may provide recruiters with a strong point to entice youth.

\section{Racial/Ethnic Differences}

While Black youth were much more likely than Whites or Hispanics to report difficulty finding jobs in their communities, military propensity decreased for Black youth since the May 2004 Youth Poll. Additionally, Black youth were more likely to report having never considered military service. Lack of employment is typically associated with increased propensity, but the negative attitudes of Black youth toward the military, particularly in terms of current events, which will be discussed in Section 3, may be counterbalancing this trend.

\section{Gender Differences}

Females were much less propensed than males and had considerably more negative attitudes toward the military, similar to Black youth. When discussing current events as well as overall military favorability in Section 3, it will be apparent that female respondents are much less tolerant of the war in Iraq and of the Bush Administration. This may be one reason females are less propensed to join the military and more propensed than men to pursue further education.

While few youth think (without being probed) that they will serve in the military after school or in the next few years, the military has at least been considered as a possible option for a substantial proportion of youth. Before taking this poll, 47\% percent of youth had given the military some consideration, while $20 \%$ had given the military serious consideration. This is good news for recruiters and should be encouraging despite declining propensity numbers. 
OVERVIEW REPORT

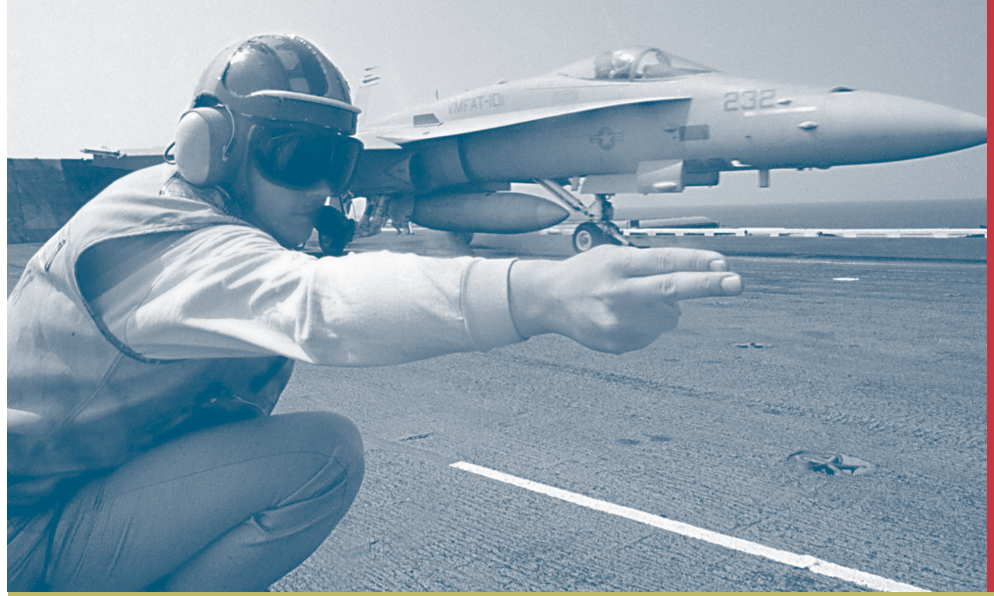

Section Three focuses on youth's attitudes toward the military, including perceived knowledge, favorability, and reactions to cument events. A discussion of youth's attitudes toward recruiters and other military outreach efforts is also provided.

\section{Section Three}




\section{PAGE LEFT BLANK INTENTIONALLY}




\section{Attitudes Toward and Communication Strategies Regarding the Military}

We know that the percentage of youth interested in the military is stable, at a low rate, but that alone does not fully explain the story. Currently, White propensity has slightly increased. It is this group that is responsible for the overall consistency in the propensity estimates. However, recent trends in propensity among minorities suggest troubling news for military recruiters as their propensity has slipped. This slip has been relatively dramatic for Black youth.

In the first part of Section 3, this report will examine youth's knowledge of and attitudes toward the U.S. Military in an effort to provide more detail and suggest possible reasons for the slip in minority propensity.

In addition, this section will incorporate youth's views on current events, as they are clearly playing a major role in youth's interest in and attitudes toward military service. Current events play a large role in shaping the perceptions of youth, but these perceptions appear to vary based on several key individual differences, including, but not limited to, race/ethnicity, gender, and age.
In addition, this section will report on the special topic covered in this Poll, communication strategies and sources of impressions and information. Where youth get their impressions of the military and where they go when seeking information about the military are two things that all professionals involved in military recruiting must understand. The source of youth's military impressions and the influence that these sources have on propensity and consideration of Military Services all affect youth's attitudes toward the military and ultimately the U.S. Military enlistment rate.

With regard to youth's attitudes and impressions of the military, the November 2004 Youth Poll asked youth about:

- favorability toward the military;

- knowledge of the military;

- military advice;

- recruiters;

- media sources;

- economic indicators; and

- perceptions of U.S. War on Terrorism.

This section provides descriptive results of questions on these and related topics. 


\section{Favorability}

To understand the general trend of youth attitudes toward the military, the Youth Polls includes a measure of youth favorability toward the military. This question asks youth to rate their overall military favorability on a 10-point scale with
10 being the highest rating. Overall, youth continue to report a generally positive view of the military, with a mean rating of 7.2. This mean rating, although positive, has dropped substantially from Youth Poll 3, conducted in November 2001.

\section{U.S. Military Favorability: By Gender}

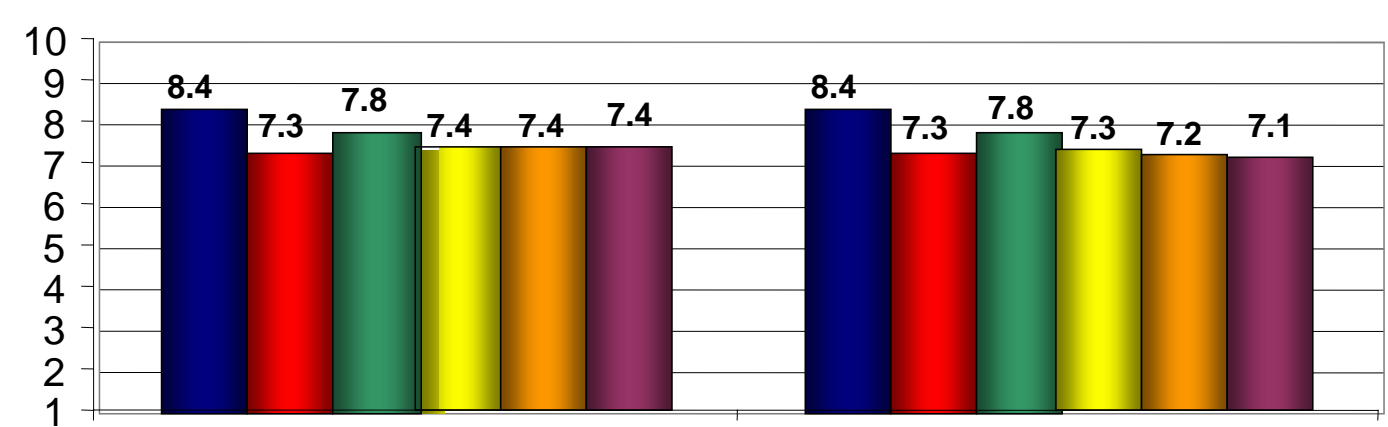

Male

Female

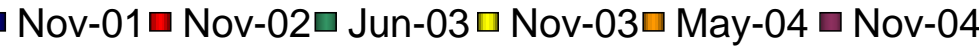

Black youth rated the military significantly less favorably than did either Whites or Hispanics. This pattern is consistent for both male and female Blacks.

Prior to the events of 9/11, the favorability ratings for Whites, Blacks, and Hispanics were similar. Since that time, Black favorability has been affected much more negatively. This indicator must continue to be watched, as it is general indicator of the problems the military is having with Black youth's military attitudes.

\section{U.S. Military Favorability: By Race/Ethnicity}

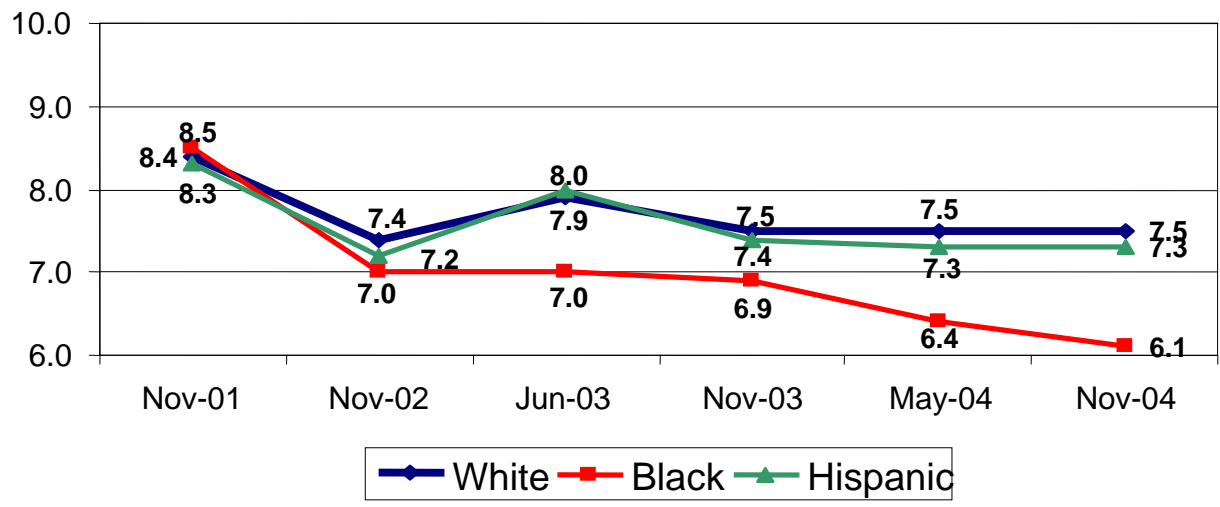

INDICATOR TO

WATCH

FAVORABILITY

AMONG BLACKS

CONTINUES TO

DECREASE 
To better understand the level of comfort and familiarity that youth have with the military, the Youth Poll also asked about self-perceived military knowledge. Youth rated their knowledge of the military on a 10-point scale, with anchors ranging from 1 -- not at all knowledgeable -- to 10 -extremely knowledgeable. Youth reported they are generally only moderately knowledgeable about the military, as evident in their mean score of 5.3. Both male and female youth have reported relatively low levels of military knowledge since this question was first asked on the November 2001 Youth Poll.

Across genders and the race/ethnicity groups, males reported feeling more knowledgeable than did females. Black males, however, felt they were less knowledgeable than both White and Hispanic males.

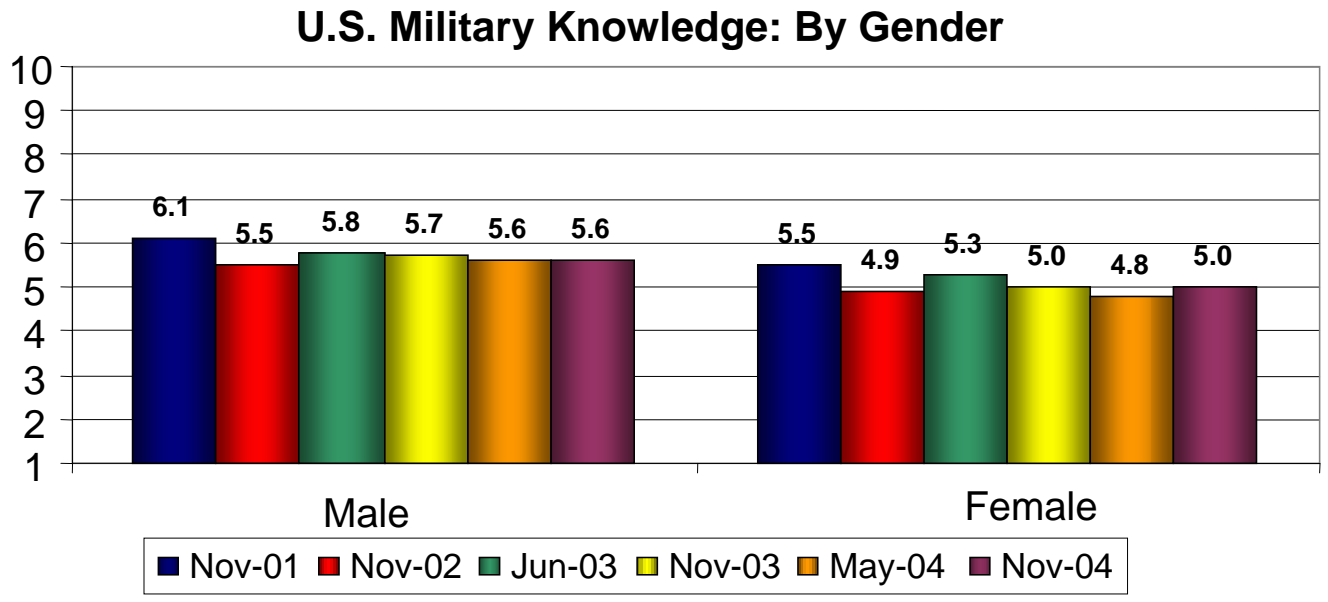

The fact that youth do not consider themselves knowledgeable about the military is somewhat concerning when considering how many of them have spoken to a military recruiter. When the November 2004 Youth Poll asked youth whether or not they had ever spoken to a military recruiter, over half $(59 \%)$ said that they had.

In contrast, in the May 2004 Youth Poll, youth were asked about different behaviors that precede future career choices. We found that while $58 \%$ of youth had either visited a company they were interested in working for or contacted a career consultant and $67 \%$ had visited a college campus, only $29 \%$ of youth said they had visited a military Web site.

In addition, of the $84 \%$ of youth who said that they had discussed their future with their parents in the last 12 months, only $34 \%$ said that the conversation included a discussion about the possibility of joining the U.S. Military.

Part of what we are seeing could be that, although youth have some knowledge of the military, they do not know that much about the military in relation to what they know about other career options. 


\section{Current Events}

The November 2004 Youth Poll asked a number of current events questions to determine the effects the War on Terrorism and the current administration's handling of international conflicts has had on youth.

\section{Troops in Iraq}

The majority of youth still support troops being in Iraq (57\%). Males were more likely to support troops in Iraq (63\%) than females $(50 \%)$. Blacks $(34 \%)$ were less likely to support troops than were Whites $(65 \%)$ or Hispanics (58\%). This is important to note as both Blacks and Hispanics have had a significant drop in their support of troops in Iraq, with Blacks having the largest drop.

$34 \%$ Proportion of Blacks who support troops being in Iraq - down from 44\% in the May 2004 Youth Poll.

\section{War on Terrorism}

The War on Terrorism continues to have a negative impact on the majority of youth. Sixty-six percent report the war has reduced their likelihood of joining the military, a significant increase since last measured in May 2004.

The War on Terrorism has had a much stronger negative effect on females and Black youth. Seventy-seven percent of females say it has made them less likely to join the military compared with only $54 \%$ of males; both of these numbers have significantly increased since last measured in May 2004.

The strength of Blacks' response to the War on Terrorism is a particular cause for concern. The large majority of Blacks (87\%, up from $80 \% 6$ months ago) report the war has reduced their likelihood of joining compared with 59\% of Whites and 69\% of Hispanics. It should be noted that the increase for both Blacks and Hispanics was significant.

$87 \%$

Proportion of Blacks who report the War on Terrorism has made them less likely to join the military

\section{Bush Administration}

Consistent with public opinion polls among adults, youth are becoming increasingly negative in their views of the Bush Administration's handling of foreign affairs and its use of military forces -- a third of youth reported strongly disapproving of each $(35 \%$ and $36 \%)$. This is a significant increase from the May 2004 findings.

Also, in line with the other current event questions asked in this poll, those who were most disapproving of Bush Administration's handling of foreign affairs and use of military forces were females and Blacks. Sixty percent of Blacks said they strongly disapproved of the handling of foreign affairs, and 58\% strongly disapproved of the way military forces are being used. In contrast, 38\% of Hispanics and 28\% of Whites reported strongly disapproving of the handling of foreign affairs, and 30\% of Whites and $40 \%$ of Hispanics strongly disapproved of how the military forces are being used.

When examined by gender, $38 \%$ of females and $31 \%$ of males strongly disapproved of how the Bush Administration is handling foreign affairs, and 53\% of females and 37\% of males strongly disapproved of how troops are being used. 


\section{Communication Strategy and Recruiters}

\section{Communication Strategy}

One factor that is critical in reaching out to inform youth about the benefits of military service is the relative efficacy of various communication strategies available. Youth Poll 8 asked youth their opinions regarding two commonly used communication strategies by recruiters: contact by phone and contact by mail.

When it comes to making important decisions, such as the decision to join the military, signaling theory is a framework that has been used to identify different communication strategies, or "signals," that are persuasive. According to signaling theory, there are costs (e.g., time, money) associated with creating any communication strategy. Different strategies can be evaluated to help identify the communication strategies that provide maximum return and minimal cost. The costs associated with phone versus mail vary greatly. A phone call allows for direct contact, but significant resources are required to reach a small number of youth. By comparison, mail is a more economical way to reach a large number of youth; however, contact is less personalized.

Youth's receptiveness was assessed by asking how likely they would be to speak with a recruiter who called their home, and how likely they would be to read military recruiter materials received via mail. These findings need to then be interpreted in light of what we know about relative costs associated with each method.

When receptiveness to phone versus mail was compared, youth reported being slightly more receptive to mail (48\%) than a phone call $(44 \%)$. This means $48 \%$ of youth would read military mail that was delivered to their home, while $44 \%$ would speak to a recruiter that called them on the phone.



Additionally, data on propensed versus nonpropensed youth and racial/ethnic differences were also prevalent. Black and Hispanic youth who were not propensed to join the military were more likely to read mail than to speak to a recruiter who called their home. Non-propensed, White youth showed no preference. In contrast, propensed youth of any race/ethnicity indicated no preference for mode of contact. It's important to keep in mind that although youth were more receptive to mail, overall, a phone call $(32 \%)$ is slightly more likely to motivate youth to have a discussion with their parents about the military than receiving a piece of mail (28\%). This may be because a phone call is more personal and creates a stronger bond between the recruiter and potential enlistee.

Given these findings, a mail campaign reaching non-propensed minority youth would likely be most effective. However, in predominately White or propensed areas, use of recruiter phone calls would be preferred because youth are just as likely to be receptive to them and they have a greater probability of being influential. 


\section{Communication Strategy and Recruiters}

Continued

\section{Recruiters}

Another factor that is critical in youth's decision to enlist in the military is youth's interaction with and perception of recruiters. According to signaling theory, potential recruits will interpret various recruitment experiences (recruiter competence, trust in recruiters, and recruitment delays) as symbolic of broader organizational characteristics. Potential recruits form impressions and images of the military quite early in the recruitment process, so it is important that the process go well from first contact. In addition, because recruiters are usually the first formal contact that youth have with the military, and because youth are usually poorly informed about the military in general, their impressions of the recruiter and their trust in the recruiter will affect how they feel about the military in general.

Because of the importance of youth's impressions of recruiters, youth were asked several questions regarding their interaction with recruiters. Questions focused on information about the recruiters themselves, as well as the recruitment techniques used by the recruiters. Overall, there is some trust in recruiters, but youth's trust in the information recruiters provide is down from when these questions were last asked in November 2002.

Only $59 \%$ percent of youth report having spoken with a military recruiter at some point in their lives. Of this $59 \%$, only $54 \%$ said that military recruiters usually present a truthful picture of military service. This means that almost half of the youth who have talked to a recruiter do not feel the recruiter presented a truthful picture of the military. Additionally, although $65 \%$ of the total youth population feel that recruiters provide information that is trustworthy, this number is down from what was found in 2002. Lastly in regard to trust in recruiters, $33 \%$ of youth feel that people entering the military often do not get the benefits promised by military recruiters. This number is up since 2002, providing even more evidence that youth's trust in recruiters has been decreasing since 2002 .

There are some positive attitudes toward recruiters that should also be pointed out. Currently, $68 \%$ of youth feel that military recruiters care about the well-being of people interested in military service. This number has increased since 2002. Although $45 \%$ of youth still feel that recruiters use high-pressure tactics to get people to join the military, this number has decreased since 2002.

Although there are some areas that recruiters seem to be improving in, there are some issues with youth's trust in recruiters. Despite the fact that the numbers for trust in recruiters are not overly low, they are down from levels of trust in 2002. Because recruiters are the frontline of military personnel and human resources, this is not good news. If the majority of youth do not trust recruiters, recruiting will become even more difficult than it is currently. 


\section{Military Advice}

\section{Conversations About the Future}

When youth were asked whether they had spoken to their parents or an educator (teachers, guidance counselors, coaches) about their plans for the future in the past 12 months, the majority said they had. Eighty four percent of youth had spoken to a parent about their future, and $56 \%$ of youth spoke to an educator.

Whites were significantly more likely to talk to their parents than were Blacks. When it came to talking to teachers, guidance counselors, and coaches, there were no significant race/ethnicity differences as to who had a conversation about their future. When asked who initiated the conversation, $79 \%$ of youth said they started the conversation with their parent, while only $64 \%$ said they initiated the conversation with an educator.

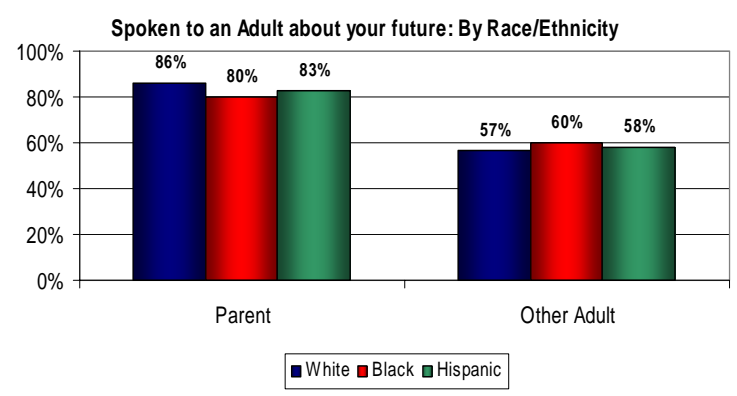

When talking to their parents about their future, the possibility of joining the military came up for only $34 \%$ of youth. In comparison, joining the military came up for only $26 \%$ of youth when talking with educators. As was reported in the DoD Educator Study (2004), educators feel pressured by administrators and parents to push college. This may be part of the reason conversations come up less frequently with educators than parents.
By race/ethnicity and gender, there are some significant differences to note. First, Blacks were significantly less likely than Whites to report conversing with their parents about the future in the last 12 months. Hispanics on the other hand, were not only likely to speak to their parents about their future, but were the most likely to have spoken to their parents about joining the military. Unfortunately, Hispanics were also more likely to report having had a negative conversation with their parents about the military than were Blacks or Whites.

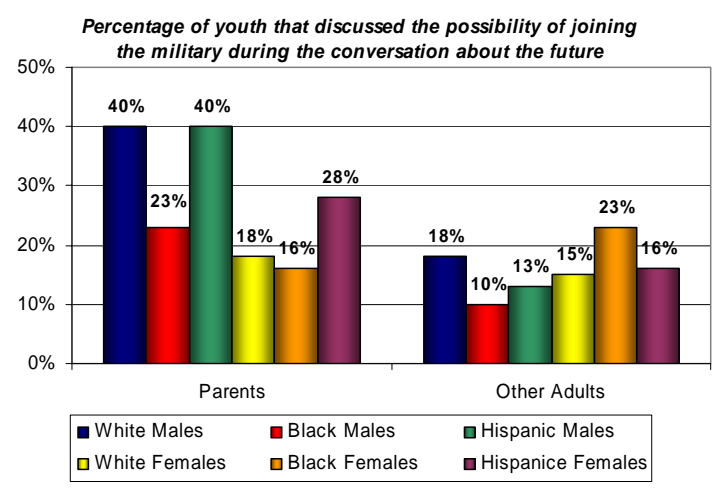

In general, when talking to parents or educators, the military was brought up as an option more often for Hispanic and White males than for Black males. This lack of military conversation between influencers and Black youth may partially explain the declines in Black male favorability toward and propensity to join the military. On the contrary, parents and educators may not talk to Black males about the possibility of a military career because they know Black youth are less interested in military service. Regardless, this is something that needs to be watched as past research has found a strong relationship between youth-influencer communications about the military and youth interest in military service. ${ }^{i}$ 


\section{Sources of Impressions}

In order to understand how youth attitudes are formed, the November 2004 Youth Poll hoped to determine what forms of information contribute to youth's impressions of the military. To achieve this, youth were asked to identify sources of their military impressions, based on a list provided that included friends, family, and media. In particular, youth were asked on a scale from 1 (not at all likely) to 5 (very likely) "How likely would you be to use

\section{Source of Impression \\ Percentage of youth that would use these source if they} were looking for information about the military

People (64\%)
Internet (63\%)

TV/Radio/Print (58\%)

Mail (43\%)

Entertainment (21\%) each source if you were looking for information regarding the military?".

Then youth were asked to assess the trust that they have in each of these sources. The results indicated that as a source, people are used as a resource most often followed by internet, media, and mail. These are also the sources that youth find most trustworthy.

\section{Trust in Source}

Percentage of youth that said they trust the information they would receive from these sources in regards to the military

\section{People $(78 \%)$}

Internet $(65 \%)$

TV/Radio/Print (60\%)

Mail (49\%)

Entertainment (16\%)

The majority of youth (64\%) report they would primarily go to people when they want information about the military. In particular, $48 \%$ of youth indicated they would go to military recruiters when looking for information about the military, while $46 \%$ indicated that they would go to a family member for military information. In addition, $42 \%$ of youth report they would go to friends for information, and $43 \%$ said they would get their information from teachers, guidance counselors, and coaches.

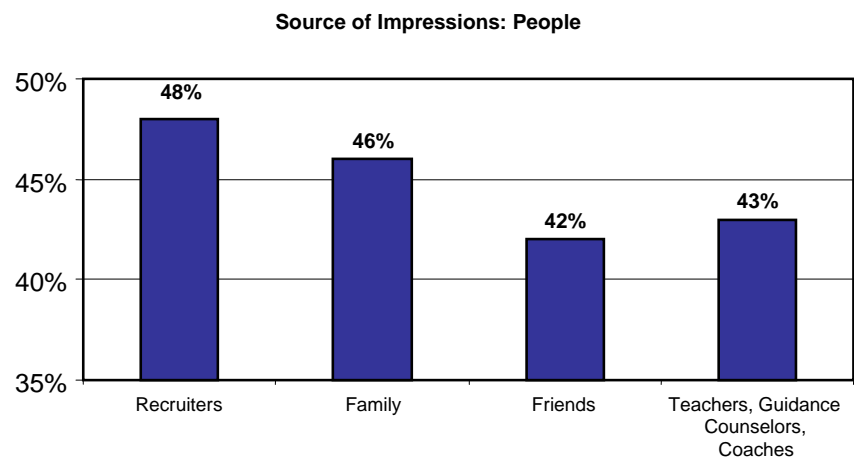




\section{Sources of Impressions}

Continued

\section{Internet}

Youth rated the Internet as the second most frequently used source when looking for information on the military. On the November 2004 Youth Poll, 61\% of youth reported using the Internet in general, and $55 \%$ reported using a military Web site for information on the military. This is good news because military personnel have worked hard to provide interesting and truthful information about the military to youth via their Web sites, and this effort seems to be paying off.

\section{$62 \%$ Proportion of youth who reported military Web sites}

\section{TV/Radio/Print}

Media outlets are another common source of youth's military impressions. Over half of youth $(58 \%)$ got the majority of their impressions of the military from the media. Overall, the top five media sources are military ads, TV news, TV commercials, newspaper articles, and magazine ads.

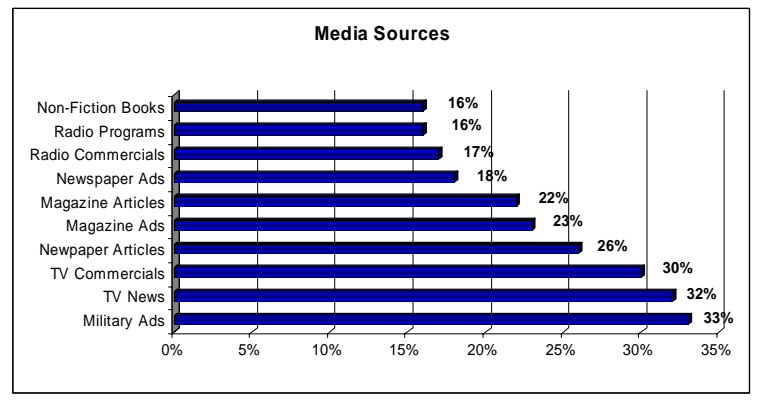

When comparing genders, the only difference is the females tend to use newspaper articles and TV news more than males. With race/ethnicity, the only group that stood out was Whites. They tend to use magazines, in general, less often than both Blacks and Hispanics.

\section{Mail}

Overall, $44 \%$ of youth said the mail they receive is a source they rely on for information about the military. Notably, there is a significant difference between males and females. Currently, $40 \%$ of females rely on mail as a source of information, while $48 \%$ of males say they rely on mail as a source of military information. Youth also find mail, like the Internet, relatively trustworthy.

\section{Entertainment}

The November 2004 Youth Poll also asked youth about information they obtain through entertainment outlets (movies, fiction, and TV entertainment programs). As has been found in the past, youth are still using sources other than entertainment to obtain most of their information about the military. Currently, only $11 \%$ use fiction books, $21 \%$ use movies, and $23 \%$ use entertainment programs. It is also important to point out that even when youth are using the sources, they do not have very much trust in the information they provide $(13 \%, 11 \%, 14 \%$, respectively).

\section{COMING SOON}

The May 2005 Youth Poll also investigates youth's sources of military impressions, but looks more deeply at where exactly youth get their information. In particular, the May 2005 Youth Poll will have specific information on which movies and TV shows youth are watching, as well as what types of impressions youth receive from different sources. 


\section{Summary}

Section 3 focused on youth's attitudes toward and impressions of the military by examining their views of the military and their attitudes toward current events. This section also looked at youth's sources of military impressions by asking where youth seek information about the military and how much trust they have in various sources.

\section{Favorability}

Currently, American youth still have favorable attitudes toward the military. Although the mean rating for favorability has gone down since first asked in November 2001, the majority of youth rate the military favorably (7.2 on a 10-point scale). By race/ethnicity, White and Hispanic favorability has remained relatively constant over poll fieldings. However, favorability for Blacks has substantially decreased. This general decline has been occurring for Blacks since June 2003 and has not yet stabilized.

\section{Knowledge}

Youth do not feel that they have a great deal of knowledge about the military, as evident in a mean rating of 5.3 on a 10-point scale. This average rating is low, but has remained relatively stable since it first dropped in November 2002. This lack of change is somewhat surprising given the level of informational coverage of military operations in the news in the last year.

\section{Current Events}

Although the majority of youth still support troops being in Iraq, the War on Terrorism is having an increasingly negative impact on youth propensity. Since the war first started, we have seen an increase in the number of youth who say the war has made them less likely to join the military. In fact, $87 \%$ of
Blacks now say the war has made them less likely to join the military, and $77 \%$ of females say the war has made them less likely to join the military.

In addition to the War on Terrorism, youth have an increasingly negative view of the Bush Administration's policies regarding foreign affairs and the use of military forces. Much like numbers from the general population, the number of youth who strongly disapprove of the Bush Administration has increased since the May 2004 Youth Poll.

\section{Communication Strategy}

When it comes to contacting youth, both mail and phone seem to be effective. Almost half of youth said that they would talk to a recruiter or read mail that came from the military. They also reported that they trust both sources of information. However, there were differences in the effectiveness of these outreach vehicles based on a youth's race/ethnicity and interest in military service. The composition of youth on these variables should be considered when planning outreach campaigns in geographical areas.

\section{Recruiters}

Although there are some areas that recruiters seem to be improving in, there are still some issues with youth's trust in recruiters. Despite the fact that the numbers for trust in recruiters are not overly low, the numbers are down from those found in 2002. Compared with two years ago, youth today are less likely to believe that people actually receive the benefits promised to them by recruiters, that recruiters provide trustworthy information, or that recruiters paint a truthful picture of the military. 


\section{Summary}

(continued)

Military Advice

The majority of youth talk to both parents and educators about their future. Unfortunately, when asked specifically about the military, less than half reported talking about the military during their discussion about the future. Coupled with past research that has shown the strong linkage between youth and parent communications and enlistment intention, this finding further highlights the advantages that could be reaped by investing in effective materials that communicate to parents and other influencers.

\section{Sources of Impressions}

Youth rely on a number of different sources when looking for information about the military. In particular, youth most often turn to people for information regarding the military. The next most frequently used source is the Internet, followed by $\mathrm{TV} /$ radio/print, and then mail. When asked about specific sources, youth said they would most likely go to recruiters for information about the military, followed by family, educators, and friends. Concerning the Internet, youth said they would go to the Internet in general as well as the military Web sites. These findings are not surprising, as youth also rated the Internet and people as their two most trusted sources.

\section{Gender Differences}

Females are much less propensed than males and have considerably more negative attitudes toward the military. Female respondents were less likely to support the American presence in Iraq, the War on Terrorism, or the Bush Administration's use of military forces and foreign policies. The War on Terrorism also has had a substantial negative effect on their likelihood of joining the military.

\section{Racial/Ethnic Differences}

Propensity has continued to decrease for Black youth since the May 2004 Youth Poll. As this is a growing trend, it could possibly interfere with the DoD's commitment to having a military service that is representative of the U.S. population along racial/ethnic lines. A key reason for this decrease is Black youth's (and influencers') negative attitudes toward current events. Black youth have demonstrated considerably lower favorability ratings of the military than Whites or Hispanics since 9/11. Black youth were less likely to support troops being in Iraq, the U.S. War on Terrorism, and the Bush Administration's use of military forces and foreign policies in general. Black youth also overwhelming reported that the War on Terrorism has made them less likely to join.

Several reasons, in addition to reactions to current events, may also help explain their recent drops in propensity. Black youth are less knowledgeable about the military and are less likely to have had a conversation about the military with their parents or an educator. Changing these factors may have a profound impact on attitudes of Black youth toward the military and may boost the military as a viable option in the face of fewer employment opportunities for Blacks outside the military. 


\section{Summary}

(continued)

In addition to these problems among Black youth, Hispanic youth are also starting to waver in their opinions of the military. This descent is something that needs to be watched closely in future polls and other tracking studies done among youth. General military propensity has gone down (although not significantly, yet) for Hispanics. In addition, although Hispanics are more likely to have had a conversation with their parents regarding the military, the conversation is least likely to have been positive. Further, recent negative shifts in reactions to current events have been most dramatic among Hispanic youth.

\section{Suggestions}

The November 2004 Youth Poll found that Blacks and females were less propensed and had more negative attitudes toward the military. However, these groups also had less knowledge of the military, fewer discussions about joining the military, and lower favorability toward the military.

Views of current events are negatively affecting youth's propensity to join the military. Because youth are using military Web sites as sources of information, explaining more about the current situation on these sites may help youth better understand the importance of current actions. In addition, positive public relations ("PR") may influence parents and educators, and may help better inform youth.
These combined findings point to a few general conclusions. First, not only youth, but also parents and influences, need more education and trustworthy information about the military. Educating these groups may lead more youth to seek information about the military, have more positive attitudes toward the military, higher favorability toward the military, and consequently increased overall propensity.

Further, youth report that family members, friends, and other people in their lives are the sources that they turn to first when looking for information. Providing youth with greater access to individuals that have served in the military and can provide real tangible examples of the benefits of military service is needed as fewer youth have immediate family members who have served.

Finally, continuing to develop an understanding of the most effective recruiting resource for a particular market is, in our opinion, needed. Maximizing the utility of recruiting investments by balancing the equation between the receptivity and relative influencing power of various outreach types for youth needs to always be the goal.

In the next section, we will continue our focus on military propensity, but will break it out by service branch and component. 
${ }^{\mathrm{i}}$ Griepentrog, B. (2005). Parental Influence on Youth Propensity: A Re-examination of the May 2004 Youth and Influencer Polls. JAMRS Executive Note, www.dmren.org 


\section{PAGE LEFT BLANK INTENTIONALLY}


OVERVIEW REPORT

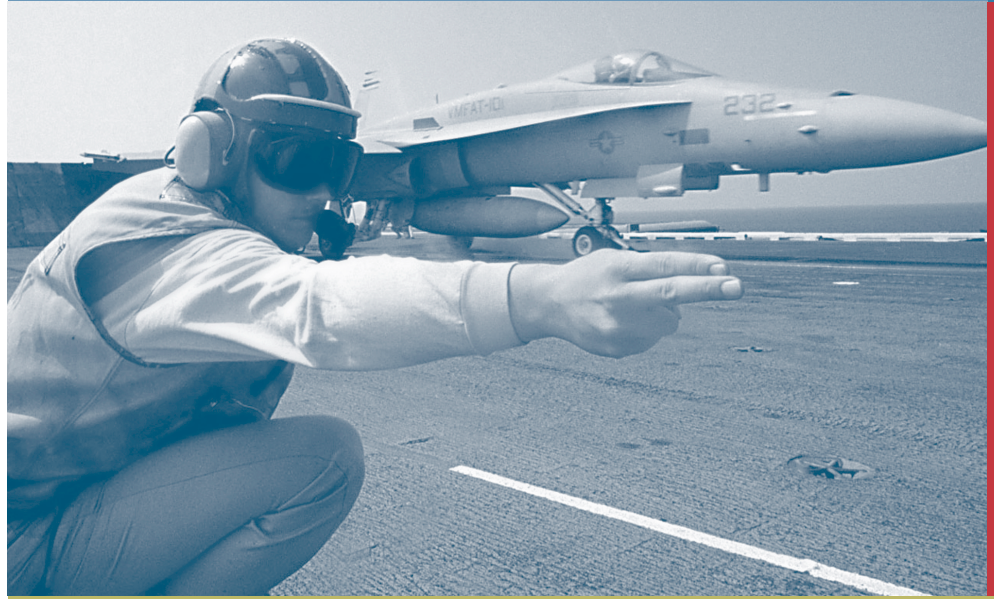

Section Four presents information on youth's favorability and propensity for each of the branches and their components. This section also presents historical trends and demographics for each Service.

\section{Section Four}




\section{PAGE LEFT BLANK INTENTIONALLY}


Since it took over for the Youth Attitude Tracking Study (YATS) in 2001, the Youth Poll has collected information from the nation's youth on their interest in serving in the U.S. Armed Forces. Sections 2 and 3 presented information on youth favorability toward, knowledge of, and propensity to enlist in the U.S. Military (overall). This section breaks down those findings further, presenting information on youth's propensity to enlist in each of the military branches and components. As in the previous sections, this section presents historical trends as well as detailed breakouts for key demographics such as gender, age, geography, and race/ethnicity.

\section{Unaided Propensity}

In Section 2, findings for the question, "What do you think you will be doing once you finish high school/once you finish college/or in the next few years?" were reported. Responses included going to school, working, and entering the military. Respondents were encouraged to indicate all the things they might be doing. Those who mentioned military service in general, or one of the services specifically, were counted as being propensed. Four percent of youth said they planned to join the military.
This is the "unaided propensity" estimate for military service that is tracked over time -"unaided" because the topic of military service is first mentioned by the respondent, not the interviewer. Of the $4 \%$ who indicated that they planned to join the military, 23\% planned on joining the Army, $18 \%$ the Navy, 21\% the Marine Corps, $29 \%$ the Air Force, and 5\% the Coast Guard. Although most of these numbers are noticeably different from the May 2004 Youth Poll, these differences are not significant due to the small sample size. From the chart below, one can see that most youth who are considered propensed tend to report being principally interested in either the Air Force, Army, or Marine Corps.

It is interesting to note that a seasonality pattern appears to be emerging for unaided Air Force propensity -- propensity has a tendency to be highest in the April through June timeframe and lowest in November. Consistent with past Youth Polls, the Coast Guard is consistently mentioned the least as the Service branch youth are interested in joining.

\section{Degree of Interest in Military Services}

\begin{tabular}{lc|c|c|c|c|c|c|c|c} 
& Apr-01 & Aug-01 & Nov-01 & Nov-02 & J & Non-03 & Nov-03 & May-04 & Nov-04 \\
\hline Army & $24 \%$ & $30 \%$ & $35 \%$ & $26 \%$ & $27 \%$ & $29 \%$ & $28 \%$ & $23 \%$ \\
\hline Navy & $21 \%$ & $18 \%$ & $12 \%$ & $15 \%$ & $13 \%$ & $17 \%$ & $12 \%$ & $18 \%$ \\
Marine Corps & $24 \%$ & $20 \%$ & $21 \%$ & $32 \%$ & $19 \%$ & $21 \%$ & $18 \%$ & $21 \%$ \\
Air Force & $31 \%$ & $24 \%$ & $26 \%$ & $26 \%$ & $35 \%$ & $25 \%$ & $35 \%$ & $29 \%$ \\
Coast Guard & $0 \%$ & $4 \%$ & $4 \%$ & $0 \%$ & $4 \%$ & $4 \%$ & $4 \%$ & $5 \%$ \\
\hline
\end{tabular}




\section{Unaided Propensity}

(continued)

Of the youth who planned to join the Army or Air Force, most (77\%) were considering Active Duty. Those not considering Active Duty, National Guard, and Reserves seemed to have a similar pattern. Both services had numbers that declined until November 2002 and then increased for a short time. Recently, it seems the Reserve propensity is down again. This may be due to the War on Terrorism.

As is true for youth propensed for the Army/Air Force, the majority of youth $(81 \%)$ planning to join the Coast Guard, Marine Corps, and Navy were considering Active Duty. This has been a consistent trend since the first Youth Poll, conducted in April 2001.

You said you would be joining the military. What type of Service would that be (ArmylAir Force)?



You said you would be joining the military. What type of Service would that be (Coast Guard/Marine Corps/Navy)?

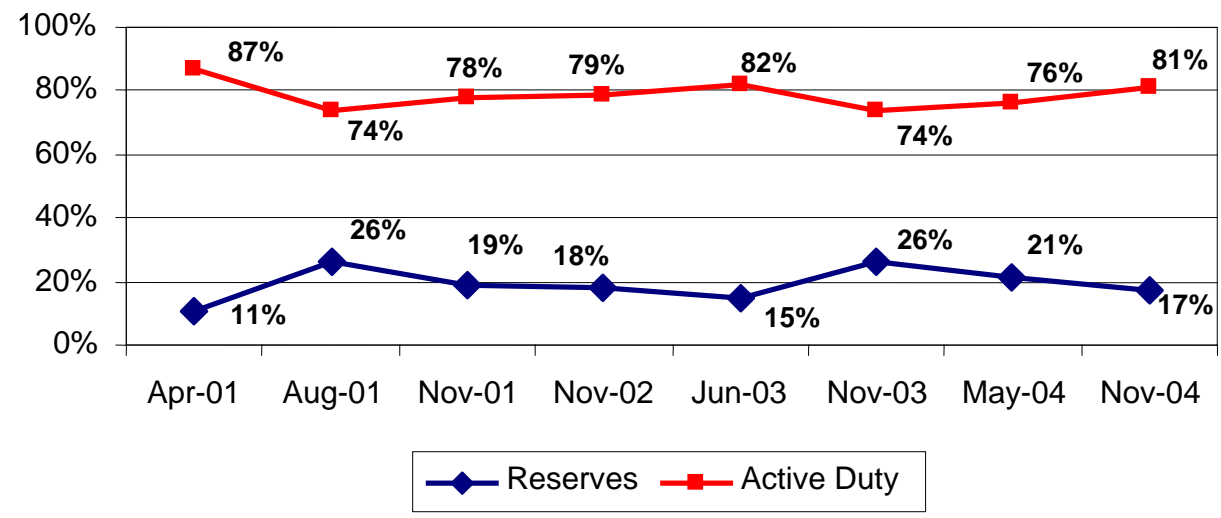




\section{Aided Propensity: Active Duty}

In addition to unaided propensity, an aided propensity measure is also tracked by the Department of Defense. Youth are asked, "How likely is it that you will be serving on Active Duty in the Army/Navy/Marine Corps/Air Force/Coast Guard?" Youth who responded that they would definitely or probably serve in a particular Service were categorized as propensed for that Service. Those who said they would probably not or definitely not serve, together with those respondents who said they don't know or who refused to answer the question, were counted as not being propensed.

Ten percent of youth reported being propensed for the Army, 8\% the Navy, 8\% the Marine Corps, $10 \%$ the Air Force, and $6 \%$ the Coast Guard. The chart below shows the poll-to-poll changes with respect to each of the active-duty services. As can be seen, most propensity numbers have remained relatively stable since the last Youth Poll done in 2004.

\section{How likely is it that you will be serving on Active Duty in the Army/Navy/Marine Corps/Air Force/Coast Guard?}

\begin{tabular}{|lc|c|c|c|c||c||c||c|c|} 
& Apr-01 & Aug-01 & Nov-01 & Nov-02 & J un-03 & Nov-03 & May-04 & Nov-04 \\
\hline Army & $9 \%$ & $7 \%$ & $10 \%$ & $9 \%$ & $10 \%$ & $11 \%$ & $10 \%$ & $10 \%$ \\
\hline Navy & $7 \%$ & $7 \%$ & $9 \%$ & $7 \%$ & $9 \%$ & $9 \%$ & $8 \%$ & $8 \%$ \\
\hline Marine Corps & $6 \%$ & $6 \%$ & $8 \%$ & $8 \%$ & $8 \%$ & $10 \%$ & $7 \%$ & $8 \%$ \\
\hline Air Force & $9 \%$ & $7 \%$ & $9 \%$ & $10 \%$ & $10 \%$ & $11 \%$ & $11 \%$ & $10 \%$ \\
Coast Guard & $4 \%$ & $5 \%$ & $6 \%$ & $6 \%$ & $6 \%$ & $6 \%$ & $6 \%$ & $6 \%$ \\
\hline
\end{tabular}




\section{Aided Propensity: Active Duty}

Breaking the numbers down by gender and military branch, there have been no significant differences for males within each branch since the May 2004 Youth Poll; however, there has been a significant decrease in female propensity for the Army since the May 2004 Youth Poll. Overall, however, male propensity continues to be significantly higher than female propensity. Both males and females continue to be most interested in either the Air Force or Army Active Duty.

\section{Propensity by Branch: Males}

\begin{tabular}{|c|c|c|c|c|c|c|c|c|}
\hline & Apr-01 & Aug-01 & Nov-01 & Nov-02 & J un-03 & Nov-03 & May-04 & Nov-04 \\
\hline Army & $10 \%$ & $10 \%$ & $16 \%$ & $13 \%$ & $14 \%$ & $15 \%$ & $13 \%$ & $14 \%$ \\
\hline Navy & $9 \%$ & $9 \%$ & $12 \%$ & $9 \%$ & $11 \%$ & $11 \%$ & $11 \%$ & $11 \%$ \\
\hline Marine Corps & $9 \%$ & $8 \%$ & $12 \%$ & $13 \%$ & $12 \%$ & $14 \%$ & $11 \%$ & $12 \%$ \\
\hline Air Force & $13 \%$ & $8 \%$ & $13 \%$ & $13 \%$ & $14 \%$ & $13 \%$ & $15 \%$ & $14 \%$ \\
\hline Coast Guard & $6 \%$ & $7 \%$ & $7 \%$ & $7 \%$ & $7 \%$ & $8 \%$ & $8 \%$ & $7 \%$ \\
\hline
\end{tabular}

Propensity by Branch: Females

\begin{tabular}{|c|c|c|c|c|c|c|c|c|}
\hline & Apr-01 & Aug-01 & Nov-01 & Nov-02 & J un-03 & Nov-03 & May-04 & Nov-04 \\
\hline Army & $4 \%$ & $5 \%$ & $5 \%$ & $5 \%$ & $6 \%$ & $7 \%$ & $7 \%$ & $5 \%$ \\
\hline Navy & $4 \%$ & $5 \%$ & $5 \%$ & $5 \%$ & $6 \%$ & $6 \%$ & $6 \%$ & $5 \%$ \\
\hline Marine Corps & $3 \%$ & $3 \%$ & $5 \%$ & $4 \%$ & $5 \%$ & $5 \%$ & $4 \%$ & $3 \%$ \\
\hline Air Force & $5 \%$ & $7 \%$ & $6 \%$ & $7 \%$ & $6 \%$ & $9 \%$ & $8 \%$ & $7 \%$ \\
\hline Coast Guard & $2 \%$ & $4 \%$ & $4 \%$ & $5 \%$ & $4 \%$ & $4 \%$ & $4 \%$ & $4 \%$ \\
\hline
\end{tabular}




\section{Aided Propensity: Reserve and National Guard}

The Youth Poll asked the same aided propensity questions for the Reserve and National Guard. The question asked was, "How likely is it that you will be serving in the Reserves/National Guard?" Fifteen percent of men reported being propensed for the Reserves, while $10 \%$ of men reported being propensed for the National Guard. Five percent of women reported being propensed for the National Guard, while $6 \%$ reported being propensed for the Reserves. There have been no significant changes in propensity for the Reserves or National Guard since last measured in May 2004.

Propensity for the National Guard and Reserves by Gender

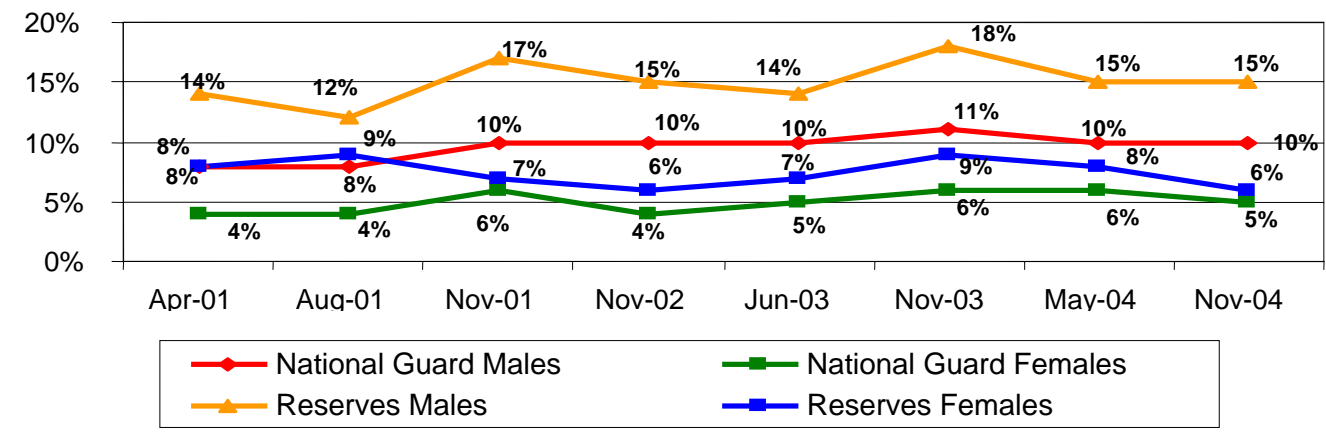

Youth who reported being propensed for the Reserves or National Guard were then asked what specific component they were considering. For those who reported being propensed for Reserves, most reported they were interested in either the Army Reserve $(32 \%)$ or the Air Force Reserve (26\%). Fewer youth reported being interested in the Marine Corps Reserve (18\%), Naval Reserve (17\%), or Coast Guard Reserve $(6 \%)$. The relative distribution across the Reserve components has not shifted significantly since last measured in May 2004.
The relative distribution across the National Guard components also has not shifted significantly since last measured. Most youth propensed for the National Guard reported being primarily interested in the Army National Guard (64\%). Only 35\% reported being interested in the Air National Guard.

When looking at gender, it is interesting to note that men overwhelmingly reported interest in the Army National Guard, while women reported almost equal interest in the Air National Guard and the Army Guard.

The remaining pages in this section present more detailed information on Army, Navy, Marine Corps, Air Force, Coast Guard, Reserves, and National Guard propensity and favorability. Propensity is broken out by gender, race/ethnicity, and geography. Overall findings are also discussed. Detailed tables can also be found in Appendix A of this report. 


\section{U.S. Army Favorability and Propensity}

Youth had a moderately positive view of the Army, as they gave it a mean rating of 7.1 on a 10-point scale. This number has remained relatively stable since the last Youth Poll in May 2004 (7.0). White males (7.3) and Hispanic and White females (7.3) rated the Army most favorably. Blacks rated the Army least favorably with a mean rating for males of 6.1 and females of 6.0 .



Army propensity is significantly higher among Hispanic males than White or Black males. Hispanics males are approximately twice as likely to be propensed as White males. Hispanic females are six times as likely to be propensed as White females. When looking at Army propensity over time, it is interesting to note that the propensity for White and Hispanic males and females has significantly increased since the first Youth Poll conducted in April 2001. In contrast, Black propensity has not significantly changed since April 2001.

Male Active Duty Propensity: Army

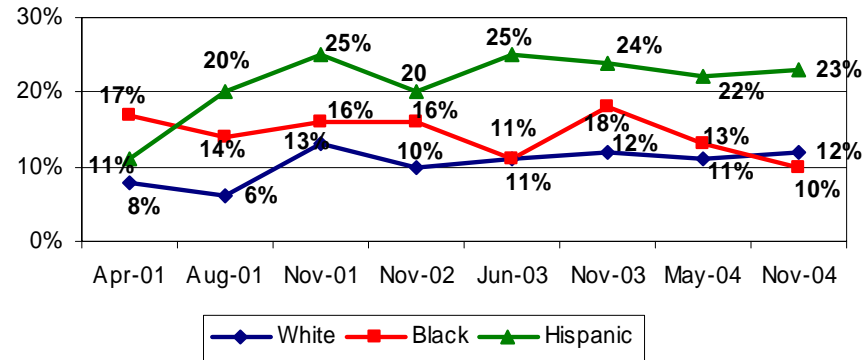

Female Active Duty Propensity: Army



For males, propensity is highest among 16-, 18-, and 20year-olds. This is inconsistent with the past Youth Polls that found male propensity highest among 16- and 17year olds. For females, propensity is highest among 16and 21-year-olds. This may be partially due to the survey population used for this study.

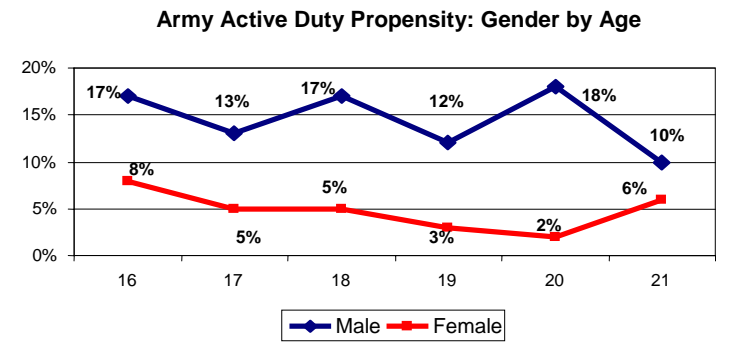

Lastly, Army propensity levels were similar in all U.S. regions except the West South Central one. This region has a very high percentage of propensed youth, which may be of interest to recruiters. The East North Central region showed the lowest level of propensed individuals for the Army at $6 \%$.

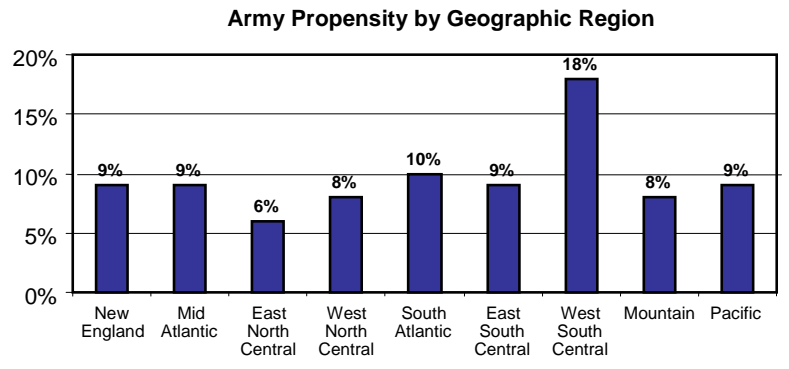




\section{U.S. Navy Favorability and Propensity}

Youth had a moderately positive view of the Navy, as they gave it a mean rating of 7.1 on a 10-point scale. This has remained statistically unchanged since the last Youth Poll in May 2004. White males (7.2), White females (7.3), and Hispanic females (7.2) rated the Navy the most favorably. Black males (6.2) and Black females (6.4)


rated the Navy least favorably. Black male favorability has significantly declined since May 2004. Also note that favorability toward the Navy for all groups has significantly decreased since this question was first asked in November 2001.

Navy propensity is significantly higher among Hispanic and Black males and females than White males and females. Additionally, when looking at the general trends, there has been a significant drop in Black female propensity since May 2004.

Male Active Duty Propensity: Navy

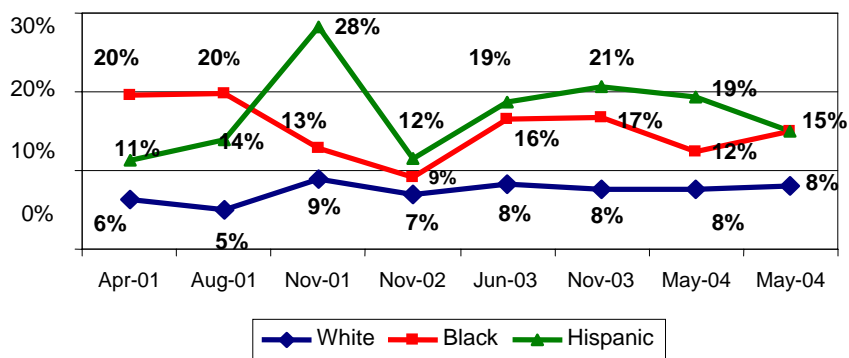

When examined by age, Navy propensity is highest among 16 and 17 year-olds. For males, propensity tends to drop off at 18 until the age of 21 . For females, propensity drops at 18 and then stabilizes.

Lastly, Navy propensity levels were similar in most regions. Propensity was lowest in the East North Central region (4\%). Propensity was higher in the Mountain (9\%) and Pacific (11\%) regions. This is consistent with findings from the May 2004 Youth Poll.
Female Active Duty Propensity: Navy

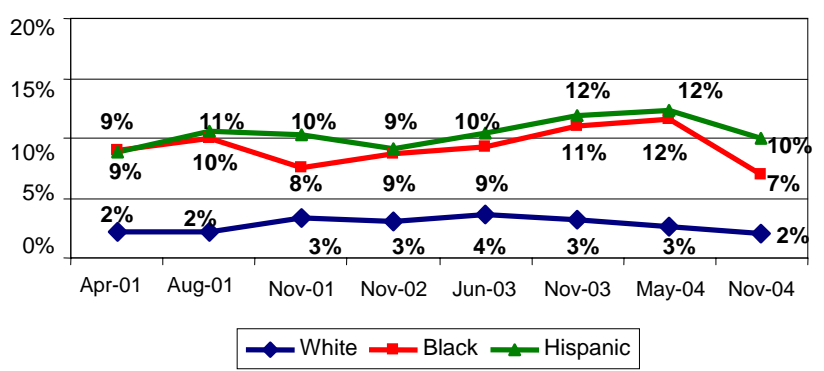

Navy Active Duty Propensity: Gender by Age



Navy Propensity by Geographic Region






\section{U.S. Marine Corps Favorability and Propensity}

Youth had a moderately positive view of the Marine Corps, as they gave it a mean rating of 7.2 on a 10 -point scale. This has remained unchanged since last measured in May 2004. Blacks rated the Marine Corps the least favorably with a mean rating of 6.1 for males and 6.0 for females. Favorability for Whites and Hispanics significantly increased in June 2003; however, this increase has now been completely lost. Favorability


toward the Marine Corps is now substantially lower than that measured in November 2001 for Whites, Blacks, and Hispanics.

Marine Corps propensity is significantly higher among Hispanics than Whites or Blacks for both males and females. In fact, Hispanic males' propensity has significantly increased since April 2001. White male propensity, although back to where it started in April 2001, is up slightly from the last Youth Poll of May 2004.
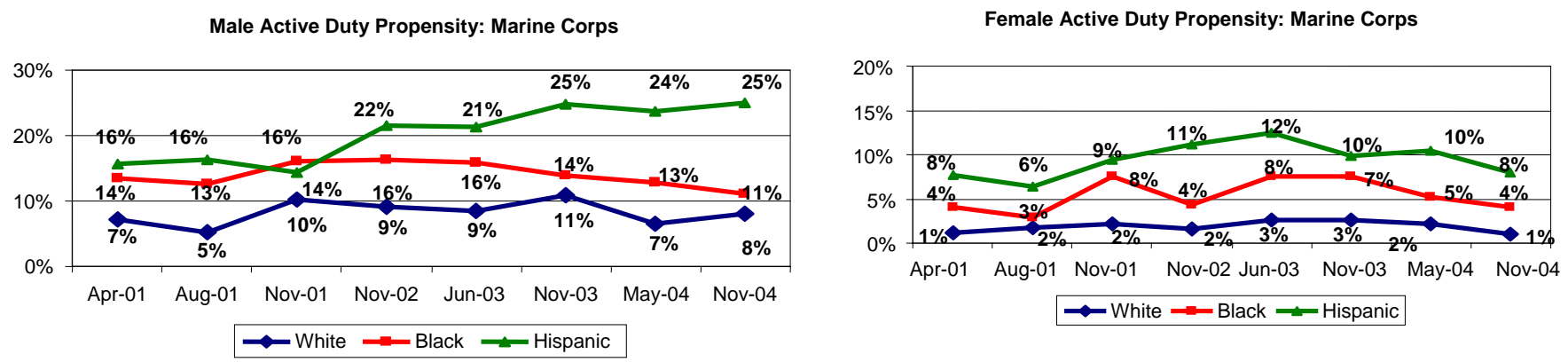

For both males and females there is a linear relationship between age and propensity, with youth who are older being less propensed. Male propensity remains relatively high until it drops off at age 21 .

As with the Army and Navy, Marine Corps propensity was lowest in the East North Central region. In addition, Marine Corps propensity was highest in the Mountain and Pacific regions.



\section{U.S. Air Force Favorability and Propensity}

Of all the Active Duty services, youth had the most positive view of the Air Force, giving it a mean rating of 7.4 on a 10-point scale. This has remained stable since November 2003. Blacks, males (6.7) and females (6.6), rate the Air Force significantly less favorably than Whites and Hispanics. As is the case for the

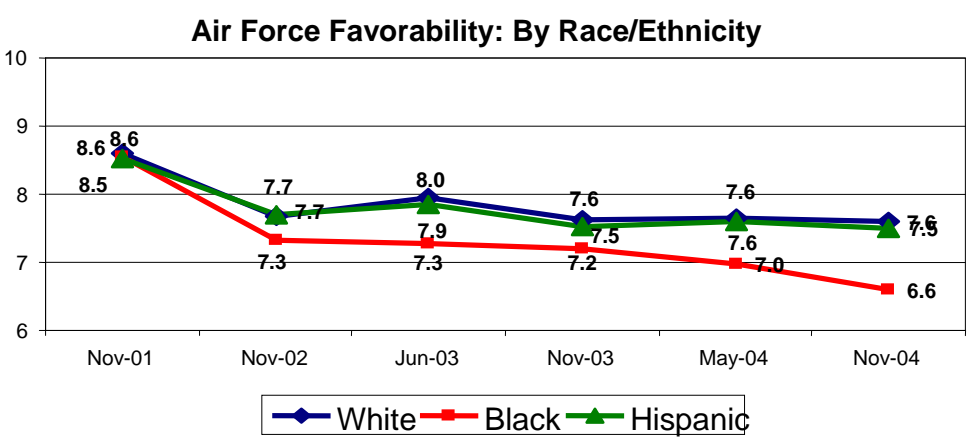
other Services, favorability toward the Air Force has significantly decreased since the Youth Poll first posed this question in November 2001.

Air Force propensity is significantly higher among male Hispanics than male Whites. Among females, Hispanics and Blacks are significantly more propensed for the Air Force than are Whites. In addition, propensity for White females has significantly dropped since May 2004.

Male Active Duty Propensity: Air Force

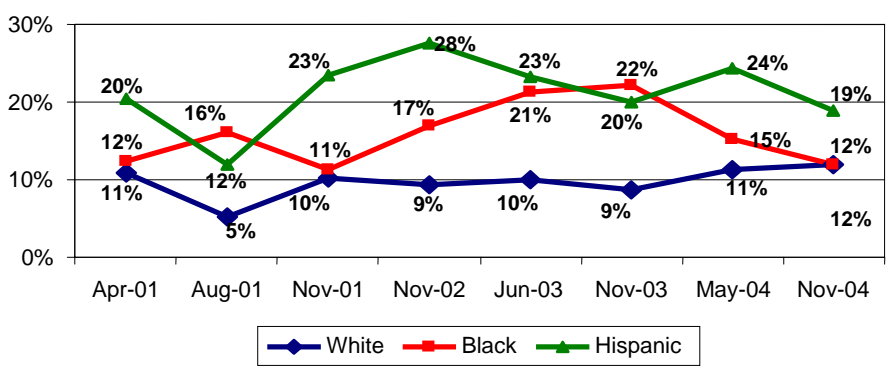

As is the case in other Active Duty branches, age is negatively related to Air Force propensity for both men and women. However, in the case of the Air Force, the drop off as youth age is less severe than it is for the other Services.

Air Force propensity is lowest in the New England region. Similar to other services, the Air Force has a strong contingent of propensed youth in the West South Central, Mountain, Pacific, and South Atlantic regions.
Female Active Duty Propensity: Air Force

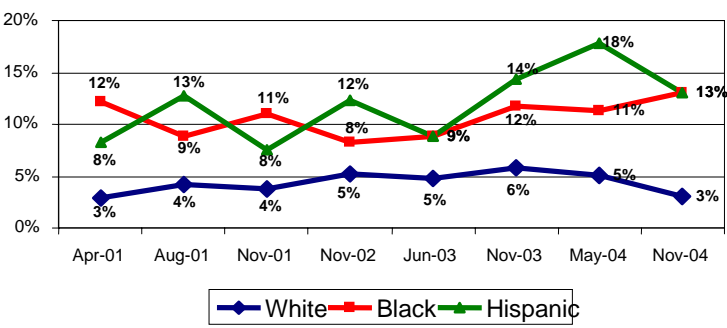

Air Force Active Duty Propensity: Gender by Age

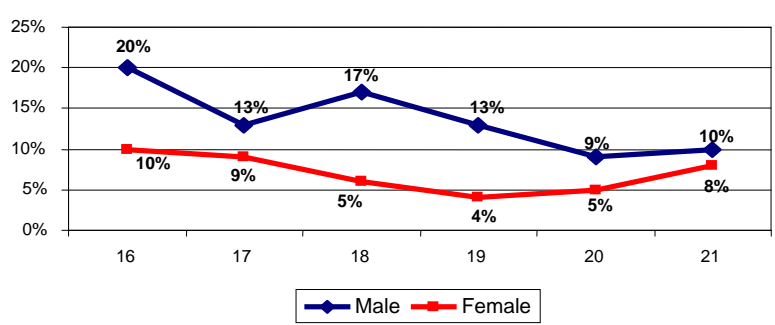

Air Force Propensity by Geographic Region

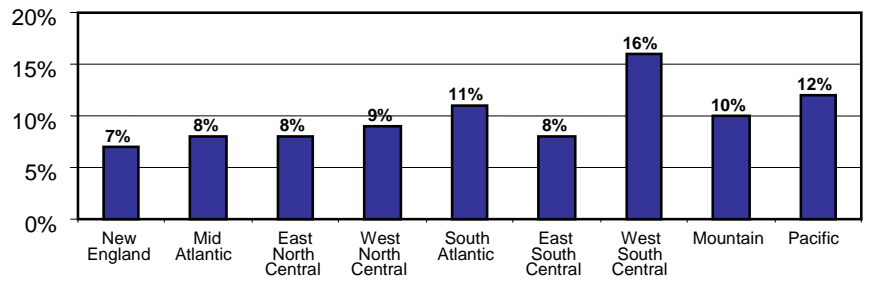




\section{U.S. Coast Guard Favorability and Propensity}

Favorability trends were lowest for the Coast Guard, with a mean favorability rating of 6.7 on a 10-point scale. This number remains consistent with the November 2003 and May 2004 Youth Polls. As with the other Active Duty services, favorability toward the Coast Guard is significantly down from November 2001. White males (7.0) and White females (7.1) rate the Coast Guard most favorably. Black males (5.7) and

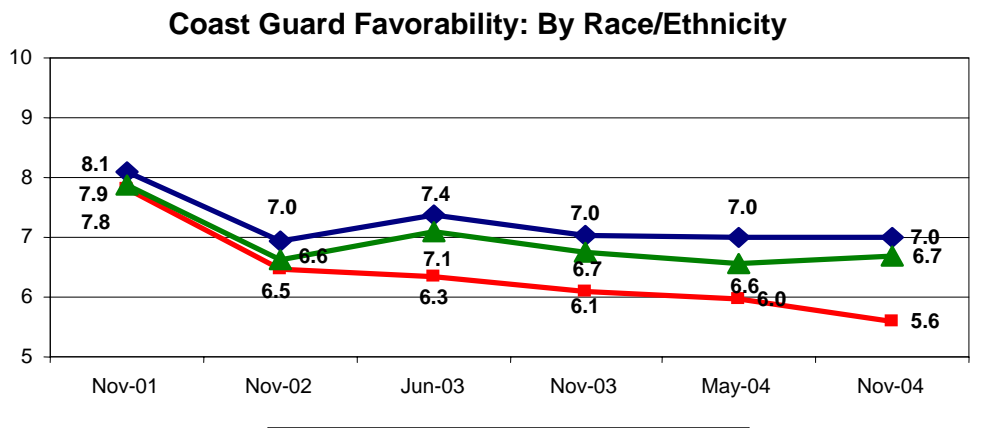
Black females (5.6) rate the Coast Guard least favorably.

Regarding the Coast Guard, propensity has not significantly changed since May 2004. As seen below, Hispanics are still more propensed for the Coast Guard than are both Blacks and Whites.

Male Active Duty Propensity: Coast Guard

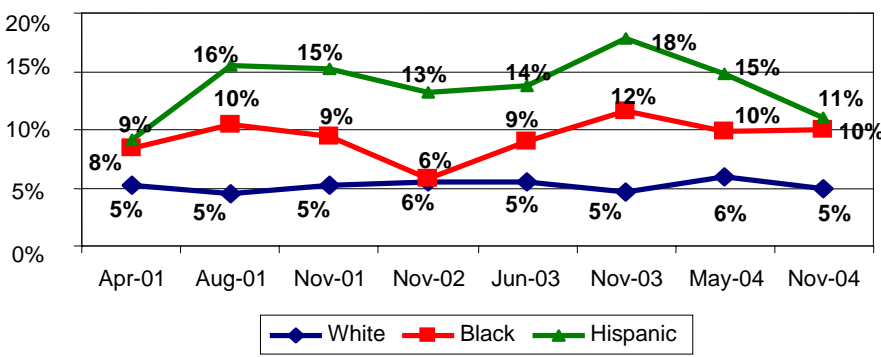

Male youth ages 16-, 17-, and 19- are the most propensed for the Coast Guard. For females, propensity is highest for 16 and 21-year-olds. This pattern of propensity is relatively unique in comparison with the other Active Duty services.

Lastly, propensity is highest in the West South Central region. Propensity is lowest in the West North Central region, with only $2 \%$ of youth propensed.

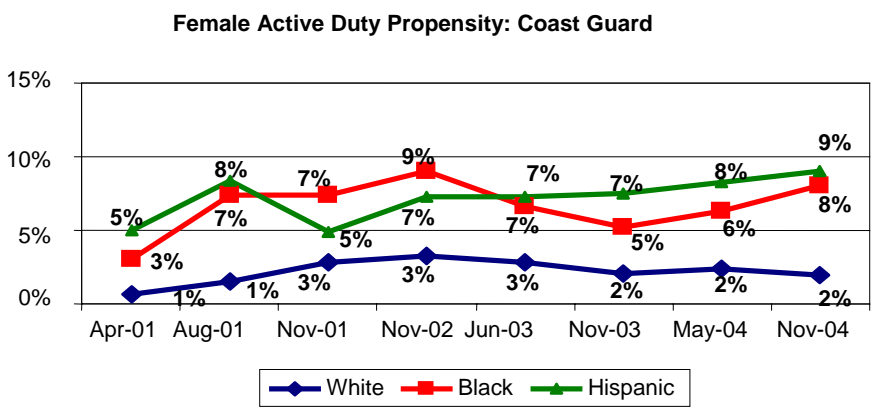

Coast Guard Active Duty Propensity: Gender by Age



Coast Guard Propensity by Geographic Region

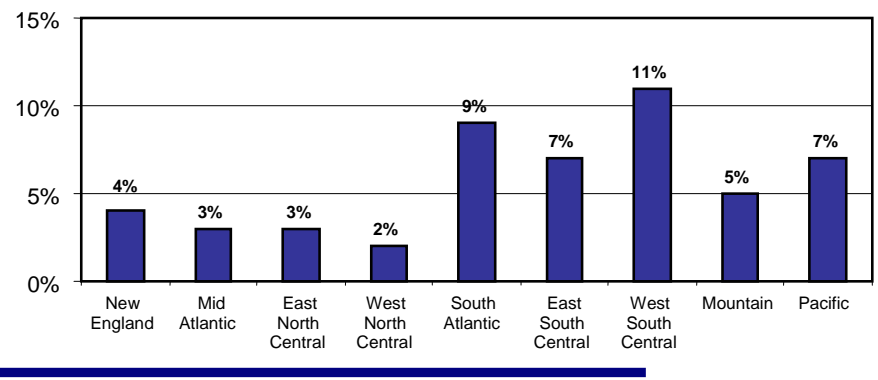




\section{U.S. Reserves Favorability and Propensity}

Overall, youth had a moderately positive view of the Reserves, as they gave it a mean rating of 6.9 on a 10-point scale. This number is relatively consistent with that measured in May 2004. Black males (5.9) and Black females (6.0) rated the Reserves the least favorably.

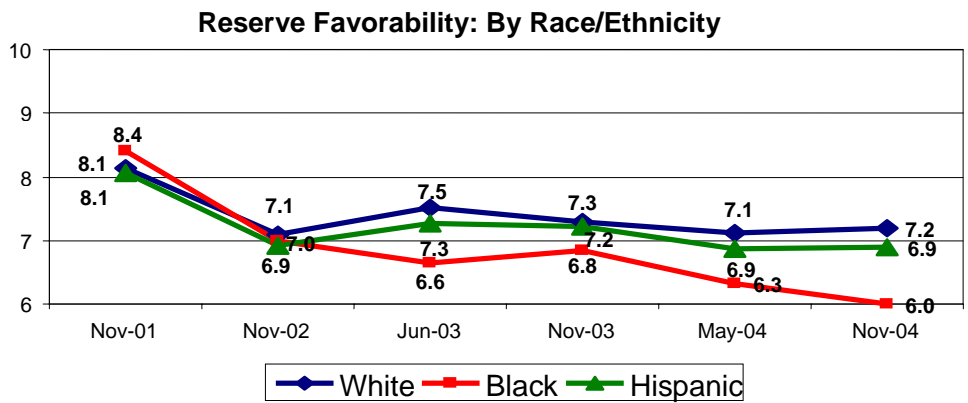

Male and female Reserve propensity is significantly higher for Hispanics than for Whites or Blacks. In fact, Hispanic female Reserve propensity is more than four times that of White females. Although White male Reserve propensity has significantly increased, both Hispanic male Reserve propensity and White female propensity have significantly decreased since last measured in May 2004.


For males, Reserve propensity was negatively related to age. The decrease was relatively gradual, with service in the Reserves remaining an option fewer youth continue to consider at the ages of 20 and 21.



Reserve Propensity by Geographic Region

Reserve propensity was consistent across most regions. New England had the lowest percentage of youth propensed for the Reserves at $7 \%$.






\section{U.S. National Guard Favorability and Propensity}

Youth had a moderately positive view of the National Guard, as they gave it a mean rating of 6.9 on a 10 -point scale. This number is relatively consistent with that measured in May 2004 (7.0). White females rated the National Guard the most favorably (7.4). Black males (6.0) and Black females (5.9) rated the National Guard the least favorably, and, overall, Black favorability is significantly down from

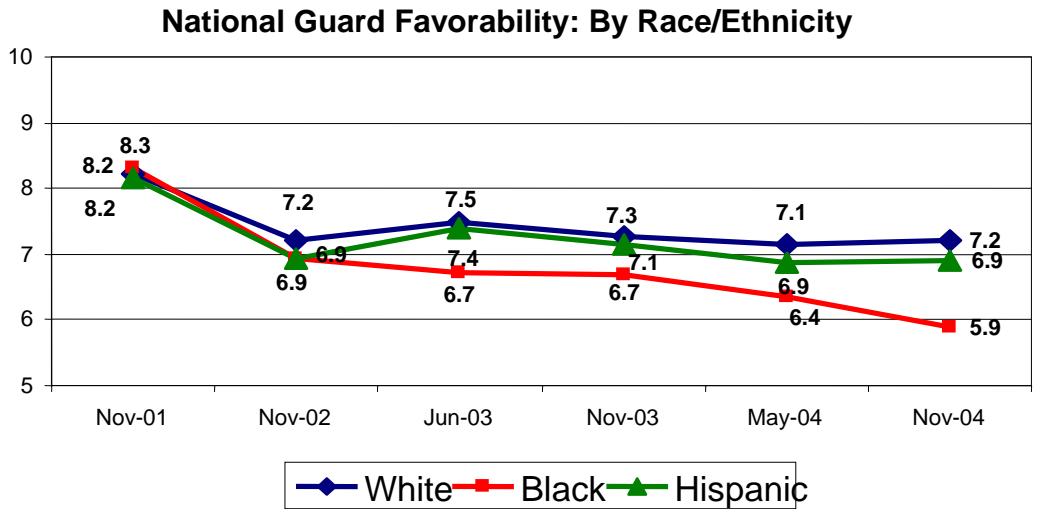
the May 2004 Youth Poll.

National Guard propensity was highest among Hispanics and lowest among Whites. Moreover, propensity for both Black (7.6) and White (1.8) females is significantly lower than that of Hispanic (12.0) females.



Although there was an overall negative relationship between age and propensity, National Guard propensity spiked at age 20 for males.

Propensity for the National Guard, similar to the Reserves, was fairly consistent across the regions. Although the West South Central region is the highest in propensity, no one region stands out.



National Guard Propensity: Gender by Age
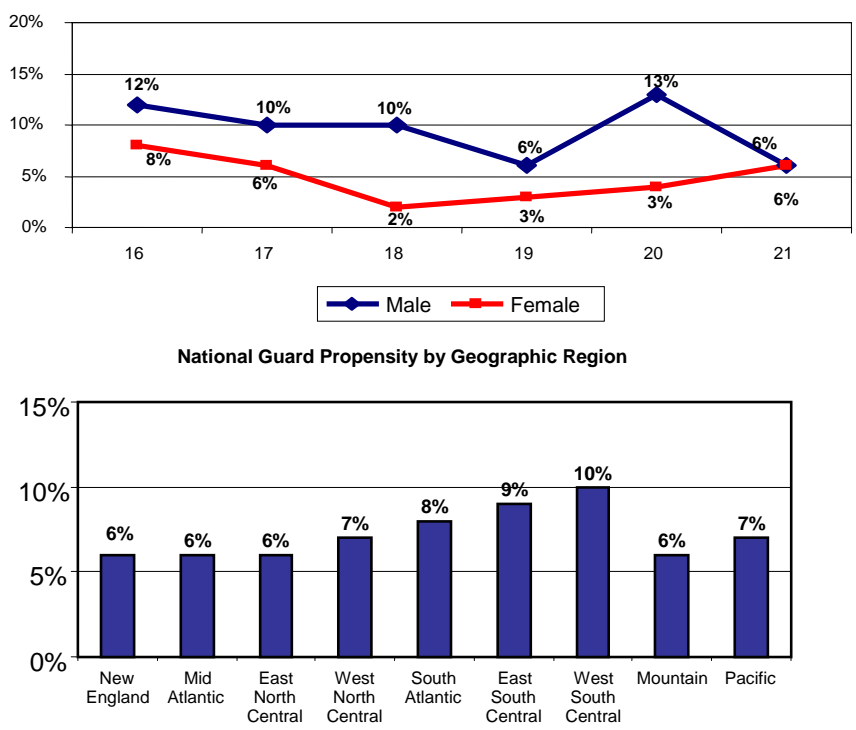


\section{Summary}

Unaided propensity for the U.S. Military has remained relatively stable at $4 \%$. Results of the November 2004 Youth Poll indicated that $10 \%$ of youth are propensed for the Army, $9 \%$ for the Navy, $8 \%$ for the Marine Corps, $10 \%$ for the Air Force, and 6\% for the Coast Guard. Overall, this does not represent a significant change from the May 2004 Youth Poll. By gender, there has been a significant decrease in female propensity for the Army.

Both Reserve and National Guard propensity have stayed relatively stable since the last Youth Poll in May 2004. Overall, $11 \%$ of youth reported being propensed for the Reserves, while 7\% reported being propensed for the National Guard. White males Reserve propensity significantly increased to $13 \%$ from $10 \%$ in May 2004. However, male Hispanic Reserve propensity and White female Reserve propensity both significantly decreased. For the National Guard, White female propensity dropped from $3 \%$ to $2 \%$, and Black female National Guard propensity dropped from $12 \%$ to $8 \%$ - both drops are significant. Moreover, Black male propensity dropped significantly from $13 \%$ to $8 \%$.

Section 4 also presented detailed findings on each of the Services' metrics, broken out by key demographic characteristics. Overall, these findings indicated a few general trends:

Favorability: Favorability toward the U.S. Military, the individual Services, the Reserves, and the National Guard has remained stable since the May 2004 Youth Poll. There has, however, been a general drop in favorability among Black youth. Although not significant across all branches, favorability among Black youth has decreased for every Service branch as well as for the military overall.

Gender: As one might expect, males tended to be more propensed than females.

Race/Ethnicity: Hispanics tended to be more propensed than either Whites or Blacks. This held for both males and females.

Age: For all branches and components, younger youth tended to be more propensed. However, there was usually an increase in propensity around age 20 or 21 for a number of the branches.

Geographic Region: For every branch and component, youth who lived in the West North Central, East North Central, or New England region were among the least likely to be propensed. It is unclear why, but the consistently lower propensity for youth who live in these regions is something worth investigating in the future. In contrast, proportionally more youth in the Mountain, Pacific, and West South Central regions were propensed.

Differences also exist in distribution of propensity across the nine regions for the specific services and components. Better understanding of these differences that could benefit recruiting. 


\section{PAGE LEFT BLANK INTENTIONALLY}


OVERVIEW REPORT

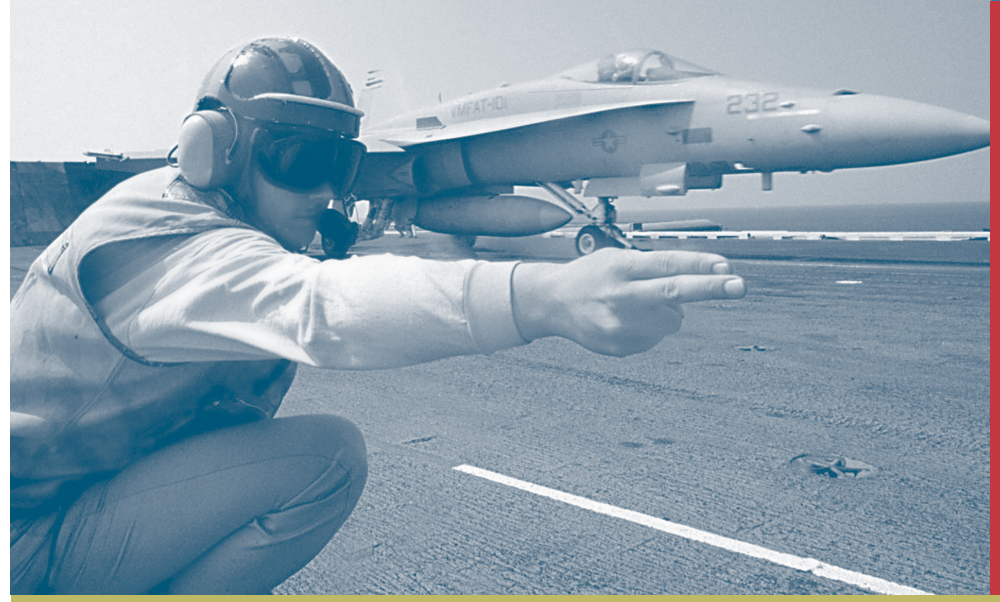

Section Five presents an overview of the findings from the November 2004 Youth Poll. Information in this section includes a summary of chapters 1-4 as well as some final recommendations.

\section{Section Five}




\section{PAGE LEFT BLANK INTENTIONALLY}




\section{Summary and Conclusions}

The November 2004 Youth Poll marked the eighth wave of the DoD's Youth Polling effort. The primary focus of the poll was to measure military enlistment intentions of youth ages 16 to 21 and to identify the sources of youth's military impressions and the influence these sources have on propensity for and consideration of military service. It is hoped that the information can be leveraged to enhance the quantity and quality of propensed American youth, thereby helping the Services meet their recruiting goals.

Each Youth Poll also tracks youth's favorability toward the military, perceived knowledge of the military, perceptions of current economic conditions, and reactions to current events.

\section{The Youth Population and Propensity}

The most dramatic social force affecting military enlistment is the interest in college attendance. Youth are focused on education and work, with the military as an after thought. Overall, only $22 \%$ of men and $7 \%$ of women said it was likely that they would serve in the military.

In regard to race/ethnicity, overall, Hispanics were the most propensed (22\%), although this percentage has dropped from the May 2004 Poll (27\%). This drop is not significant; however, it is something that should be monitored. Black propensity did drop significantly falling from $16 \%$ on the May 2004 Poll to $11 \%$ on this current Youth Poll.
Youth propensity to serve on Active Duty in each of the individual branches is similar to that found in the May 2004 Youth Poll. Ten percent of youth reported being likely to serve in the Army, $8 \%$ in the Navy, $8 \%$ in the Marine Corps, $10 \%$ in the Air Force, and $6 \%$ in the Coast Guard.

Reserve and National Guard propensity have remained relatively stable since the May 2004 Youth Poll as well. Overall, 12\% of youth were propensed for the Reserves, while $8 \%$ were propensed for the National Guard. Broken out by race and gender, however, there are some significant changes. Black propensity for the Reserves, as well as female propensity for the Reserves, has significantly decreased. This is part of the continuing downward trend that has been observed for both Blacks' and females' interest in military service.

Propensity also meaningfully varied by other demographic segments:

- Gender: Propensity for men was higher than for women for all Services.

- Age: The younger the person the greater their propensity, with a spike around age 20.

- Race: Hispanics were most propensed.

- Geographic Region: Youth in the Mountain, Pacific, and West South Central region had the highest propensity of all U.S. regions.

- Employment status: Unemployed youth had higher propensity than did youth who were employed. 


\section{Summary and Conclusions}

(continued)

Youth's Attitudes Toward the Military

Youth reported an overall positive view of the military, although they admitted that they are not very knowledgeable about it. The mean favorability rating was 7.2 , while the mean knowledge rating was 5.3 on scales from 1 (low) to 10 (high). With regard to youth favorability toward the Services and components, the Air Force received the highest mean rating (7.4).

Not surprisingly, the War on Terrorism has had an effect on youth's likelihood of joining the military. The majority of youth now report feeling that the country is heading in the wrong direction. Further, minorities and women appear to have an increased level of discontentment about both the war and the Bush Administration.

When asked about the War on Terrorism, $66 \%$ of youth reported that they were less likely to join the military as a result. This is up from 59\% on the May 2004 Youth Poll. Notably, the groups most negatively affected were women and Blacks. Seventy-seven percent of women said it has made them less likely to join the military, and a large majority of Blacks (87\%) reported the war has resulted in them being less likely to join.

In addition, $69 \%$ of Hispanics say the War on Terrorism has made them less likely to join the military, up from 57\% in May 2004. This represents the most sizeable increase from May 2004. That increase, coupled with marginally significant drops in propensity for Hispanics on this poll, indicates, in our view, increased problems of recruiting Hispanics on the horizon. We believe increased attention needs to be paid to this group immediately before attitudes and propensity drops are seen that are similar to those recently observed for African Americans. If nothing is done to address these groups' core attitude shifts, we expect that recruiting problems will worsen, regardless of the monetary incentives offered.

\section{Communication Strategy}

Youth receptiveness to phone versus mail was compared on the November 2004 Youth Poll. Results indicate that effectiveness of outreach campaigns depends on race/ethnicity variables, as well as propensity. Overall, $48 \%$ of youth are likely to read recruiting materials that come in the mail, and $44 \%$ are likely to speak with a recruiter who calls on the phone. Mail was particularly effective for reaching nonpropensed minority youth. However, direct recruiter contact, as expected, was reported by youth to have a stronger effect on their behavior.

\section{Recruiters}

Youth generally have a higher level of mistrust about what recruiters say and promise than they did 2 years ago. Youth think that the picture that recruiters are painting is less trustworthy and that recruiters provide less truthful information compared to youth perceptions in 2002. Additionally, more youth feel that people are not getting the benefits promised to them by recruiters. Dwindling trust of recruiters is a major concern as they represent the frontline of military recruiting. 


\section{Summary and Conclusions}

(continued)

\section{Youth's Sources of Impressions}

Over half of youth reported that people and the Internet were the major sources of their impressions of the military. These sources are followed in influence by TV/radio/print and mail.

Specifically, youth reported that recruiters, followed by family, educators, and friends, were the means they currently use when they need information about the military. It is refreshing to see that youth turn to recruiters first when looking for information regarding the military. This does, however, reinforce the importance of ensuring that the information recruiters provide continues to be seen as valued and trustworthy.

Overall, youth reported that family is also a major source of military impressions for them. However, as expected, family is not as supportive of the military. When asked about the conversations they had with their parents about the military, only $38 \%$ of youth said they received positive responses from their parents regarding the military. In contrast, when discussing the military as an option with educators, $58 \%$ of youth said that educators were positive about the discussion.

In terms of delivering specific information to youth about the military in the future, people and the media appear to be strong conduits. Over half of youth reported that media sources such as the Internet or television are channels they use for specific information about the military.
Factors that Influence Youth Propensity Youth attitudes toward and knowledge of the military were factors that influenced youth propensity for the military. Youth who rated the military more favorably were more propensed. Also, youth who rated themselves as more knowledgeable about the military were more likely to join it.

\section{Moving Forward}

Examining the demographics and goals of youth helps recruiters identify some potential ways to increase the effectiveness of recruitment efforts. This report, in addition to looking at propensity, provides insight into youth attitudes, the sources of military impressions, the influence of people with whom youth associate personally, and the support they believe they would receive if they were to join the military. These insights can be examined and used in future communications campaigns directed toward youth.

In addition, recruiters can take this information and hopefully influence those that have the greatest effect on youth's decision-making. Building the support of these influencers will create more direct communication channels that the U.S. Military can use to enhance military favorability and knowledge, and to ultimately increase military propensity among American youth. 


\section{PAGE LEFT BLANK INTENTIONALLY}


OVERVIEW REPORT

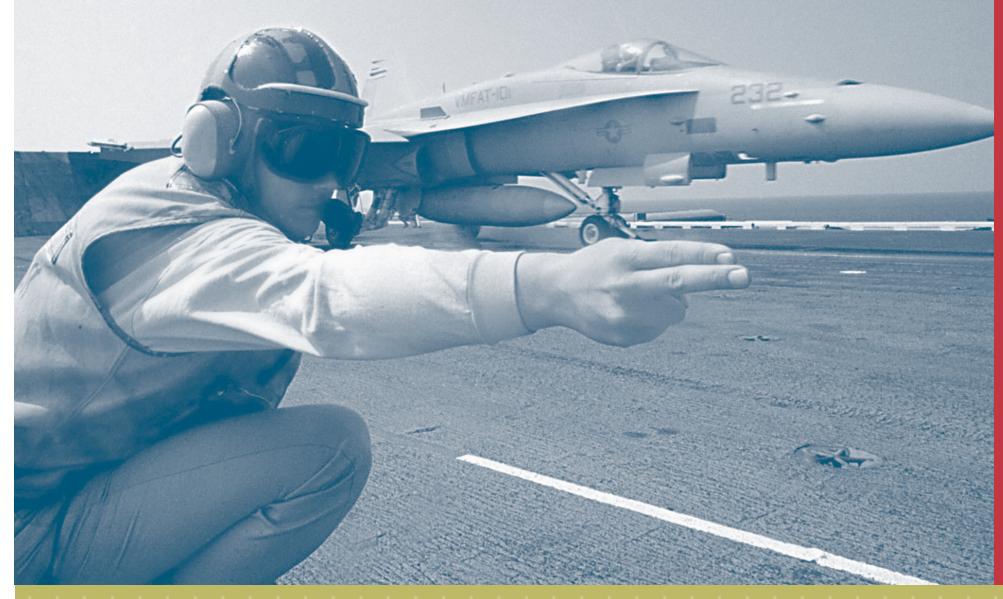

Appendix A 


\section{PAGE LEFT BLANK INTENTIONALLY}




\section{General Military Propensity}

TABLE 1-1. Youth military service propensity: $2001-2004^{1}$

Male and Female (age 16-21)

Percent (\%)

\begin{tabular}{lcccc}
\cline { 2 - 4 } Year & Definitely & Probably & Probably Not & Definitely Not \\
\hline Youth Poll 1 (Apr 01) & 2.4 & 9.5 & 26.5 & 61.5 \\
Youth Poll 2 (Aug 01) & 2.3 & 9.7 & 29.1 & 58.1 \\
Youth Poll 3 (Nov 01) & 2.8 & 11.8 & 33.3 & 52.1 \\
Youth Poll 4 (Nov 02) & 2.5 & 10.7 & 30.6 & 56.2 \\
Youth Poll 5 (June 03) & 3.5 & 11.8 & 30.5 & 54.0 \\
Youth Poll 6 (Nov 03) & 3.3 & 13.2 & 30.8 & 52.6 \\
Youth Poll 7 (May 04) & 3.2 & 11.6 & 32.4 & 52.6 \\
Youth Poll 8 (Nov 04) & 3.0 & 11.2 & 31.2 & 54.4 \\
\hline
\end{tabular}

Male (age 16-21)

Percent (\%)

\begin{tabular}{lcccc}
\cline { 2 - 4 } Year & Definitely & Probably & Probably Not & Definitely Not \\
\hline Youth Poll 1 (Apr 01) & 3.6 & 14.2 & 34.4 & 47.6 \\
Youth Poll 2 (Aug 01) & 3.4 & 12.0 & 37.1 & 47.5 \\
Youth Poll 3 (Nov 01) & 4.5 & 18.5 & 40.8 & 36.3 \\
Youth Poll 4 (Nov 02) & 3.8 & 14.9 & 35.2 & 46.0 \\
Youth Poll 5 (June 03) & 5.3 & 17.0 & 35.7 & 41.6 \\
Youth Poll 6 (Nov 03) & 5.4 & 17.9 & 37.9 & 38.6 \\
Youth Poll 7 (May 04) & 4.6 & 15.5 & 38.8 & 40.7 \\
Youth Poll 8 (Nov 04) & 5.0 & 16.6 & 37.3 & 41.0 \\
\hline
\end{tabular}

Female (age 16-21)

Percent (\%)

Year

Definitely Probably $\quad$ Probably Not $\quad$ Definitely Not

Youth Poll 1 (Apr 01)

1.3

5.1

19.1

74.3

Youth Poll 2 (Aug 01)

1.4

7.6

21.5

69.3

Youth Poll 3 (Nov 01)

1.2

5.6

26.2

67.0

Youth Poll 4 (Nov 02)

1.3

6.6

26.1

66.0

Youth Poll 5 (June 03)

1.6

6.4

25.2

66.6

Youth Poll 6 (Nov 03)

1.2

8.5

23.6

66.7

Youth Poll 7 (May 04)

1.7

7.7

25.8

64.8

Youth Poll 8 (Nov 04)

1.0

5.7

24.9

68.2

\footnotetext{
${ }^{1}$ ‡Reporting standard not met (too few cases).

Note: Changes in sample size and stratification were implemented following November 2002 Youth Poll.

Source: Department of Defense Polls, JAMRS, 2001-2004 (Question: FPP9).
} 


\section{General Military Propensity}

TABLE 1-2. Youth military service propensity, by racelethnicity: $2001-2004^{2}$

\begin{tabular}{|c|c|c|c|c|}
\hline \multirow{3}{*}{$\begin{array}{l}\text { Male and Female (age 16-21) } \\
\text { (definitely \& probably) } \\
\text { Year }\end{array}$} & \multicolumn{4}{|c|}{ Percent (\%) } \\
\hline & \multicolumn{4}{|c|}{ Race/ethnicity } \\
\hline & Total & White & Black & Hispanic \\
\hline Youth Poll 1 (Apr 01) & 11.9 & 10.4 & 16.1 & 14.3 \\
\hline Youth Poll 2 (Aug 01) & 12.1 & 8.6 & 16.6 & 19.9 \\
\hline Youth Poll 3 (Nov 01) & 14.6 & 11.9 & 14.1 & 26.9 \\
\hline Youth Poll 4 (Nov 02) & 13.2 & 9.7 & 14.8 & 23.5 \\
\hline Youth Poll 5 (June 03) & 15.3 & 12.5 & 16.0 & 24.7 \\
\hline Youth Poll 6 (Nov 03) & 16.5 & 13.0 & 20.7 & 24.8 \\
\hline Youth Poll 7 (May 04) & 14.8 & 11.3 & 16.0 & 27.0 \\
\hline Youth Poll 8 (Nov 04) & 14.2 & 12.0 & 10.9 & 22.4 \\
\hline Male (age 16-21) & \multicolumn{4}{|c|}{ Percent (\%) } \\
\hline (definitely \& probably) & \multicolumn{4}{|c|}{ Race/ethnicity } \\
\hline Year & Total & White & Black & Hispanic \\
\hline Youth Poll 1 (Apr 01) & 17.8 & 17.1 & 20.9 & 18.0 \\
\hline Youth Poll 2 (Aug 01) & 15.3 & 11.4 & 22.0 & 23.3 \\
\hline Youth Poll 3 (Nov 01) & 23.0 & 19.9 & 21.1 & 37.2 \\
\hline Youth Poll 4 (Nov 02) & 18.7 & 15.1 & 17.6 & 33.1 \\
\hline Youth Poll 5 (June 03) & 22.3 & 18.4 & 23.4 & 35.8 \\
\hline Youth Poll 6 (Nov 03) & 23.2 & 20.1 & 25.6 & 31.6 \\
\hline Youth Poll 7 (May 04) & 20.1 & 16.4 & 20.5 & 32.3 \\
\hline Youth Poll 8 (Nov 04) & 21.6 & 19.4 & 16.8 & 28.2 \\
\hline Female (age 16-21) & \multicolumn{4}{|c|}{ Percent (\%) } \\
\hline (definitely \& probably) & \multicolumn{4}{|c|}{ Race/ethnicity } \\
\hline Year & Total & White & Black & Hispanic \\
\hline Youth Poll 1 (Apr 01) & 6.4 & 3.8 & 12.8 & 11.1 \\
\hline Youth Poll 2 (Aug 01) & 9.0 & 5.8 & 13.2 & 16.3 \\
\hline Youth Poll 3 (Nov 01) & 6.8 & 3.8 & 10.1 & 15.9 \\
\hline Youth Poll 4 (Nov 02) & 7.9 & 4.4 & 12.0 & 16.1 \\
\hline Youth Poll 5 (June 03) & 8.0 & 6.2 & 9.9 & 13.1 \\
\hline Youth Poll 6 (Nov 03) & 9.7 & 5.4 & 16.6 & 18.2 \\
\hline Youth Poll 7 (May 04) & 9.4 & 5.7 & 12.0 & 21.6 \\
\hline Youth Poll 8 (Nov 04) & 6.7 & 3.8 & 6.7 & 16.4 \\
\hline
\end{tabular}

\footnotetext{
${ }^{2}$ Due to relatively small sample sizes, American Indian, Alaska Native, Asian, and Pacific Islander are included in the total but are not shown separately. Black includes African American, and Hispanic includes Latino. Race categories exclude Hispanic origin unless specified otherwise.

‡Reporting standard not met (too few cases).

Note: Changes in sample size and stratification were implemented following November 2002 Youth Poll. Source: Department of Defense Polls, JAMRS, 2001-2004 (Question: FPP9).
} 


\section{General Military Propensity}

TABLE 1-3. Youth military service propensity, by age: $2001-2004^{3}$

\begin{tabular}{lccccccc} 
Male and Female (age 16-21) & \multicolumn{7}{c}{ Percent (\%) } \\
\cline { 2 - 8 } $\begin{array}{l}\text { (definitely \& probably) } \\
\text { Year }\end{array}$ & $\mathbf{1 6}$ & $\mathbf{1 7}$ & $\mathbf{1 8}$ & $\mathbf{1 9}$ & $\mathbf{2 0}$ & $\mathbf{2 1}$ \\
\hline & 19.8 & 17.0 & 11.9 & 8.2 & 6.7 & 7.7 \\
\hline Youth Poll 1 (Apr 01) & 21.2 & 15.4 & 13.2 & 6.8 & 11.5 & 3.8 \\
Youth Poll 2 (Aug 01) & 20.9 & 21.7 & 12.3 & 9.4 & 10.3 & 12.9 \\
Youth Poll 3 (Nov 01) & 19.9 & 16.8 & 10.8 & 12.2 & 12.7 & 6.0 \\
Youth Poll 4 (Nov 02) & 23.3 & 17.7 & 16.6 & 14.2 & 10.8 & 8.3 \\
Youth Poll 5 (June 03) & 26.3 & 20.8 & 15.4 & 12.1 & 13.0 & 9.4 \\
Youth Poll 6 (Nov 03) & 22.0 & 19.7 & 14.3 & 11.9 & 9.1 & 10.4 \\
Youth Poll 7 (May 04) & 20.4 & 17.7 & 13.8 & 13.0 & 10.9 & 8.5 \\
Youth Poll 8 (Nov 04) & & & & & & \\
\hline
\end{tabular}

\section{Male (age 16-21)}

(definitely \& probably)

\section{Year}

Youth Poll 1 (Apr 01)

Youth Poll 2 (Aug 01)

Youth Poll 3 (Nov 01)

Youth Poll 4 (Nov 02)

Youth Poll 5 (June 03)

Youth Poll 6 (Nov 03)

Youth Poll 7 (May 04)

Youth Poll 8 (Nov 04)

\begin{tabular}{cccccc}
\multicolumn{7}{c}{ Percent (\%) } \\
\hline \multicolumn{7}{c}{ Age } \\
\hline $\mathbf{1 6}$ & $\mathbf{1 7}$ & $\mathbf{1 8}$ & $\mathbf{1 9}$ & $\mathbf{2 0}$ & $\mathbf{2 1}$ \\
\hline 25.3 & 23.0 & 18.4 & 15.0 & 9.2 & 14.2 \\
25.4 & 20.9 & 14.2 & 10.9 & 15.3 & 1.0 \\
31.5 & 29.0 & 19.8 & 17.7 & 13.1 & 25.3 \\
23.6 & 26.5 & 15.9 & 19.0 & 19.1 & 5.5 \\
33.1 & 22.1 & 23.7 & 22.8 & 17.8 & 13.7 \\
33.0 & 26.9 & 23.6 & 18.5 & 21.4 & 13.9 \\
29.3 & 26.9 & 20.4 & 14.6 & 14.4 & 13.1 \\
28.7 & 26.5 & 21.7 & 20.2 & 18.9 & 11.7 \\
\hline
\end{tabular}

\section{Female (age 16-21)}

(definitely \& probably)

Year

Youth Poll 1 (Apr 01)

Youth Poll 2 (Aug 01)

Youth Poll 3 (Nov 01)

Youth Poll 4 (Nov 02)

Youth Poll 5 (June 03)

Youth Poll 6 (Nov 03)

Youth Poll 7 (May 04)

Youth Poll 8 (Nov 04)

\begin{tabular}{cccccc}
\multicolumn{7}{c}{ Percent (\%) } \\
\hline $\mathbf{7}$ & Age \\
\hline 16 & $\mathbf{1 7}$ & $\mathbf{1 8}$ & $\mathbf{1 9}$ & $\mathbf{2 0}$ & $\mathbf{2 1}$ \\
14.5 & 10.0 & 6.1 & 1.1 & 4.5 & 3.2 \\
16.0 & 9.6 & 12.3 & 3.2 & 8.3 & 5.9 \\
10.8 & 13.6 & 4.8 & 2.7 & 8.0 & 1.1 \\
15.8 & 7.7 & 5.5 & 6.1 & 5.8 & 6.4 \\
13.3 & 13.0 & 9.3 & 5.6 & 3.1 & 3.2 \\
19.5 & 14.1 & 7.4 & 5.6 & 5.1 & 4.9 \\
14.5 & 12.8 & 7.3 & 9.1 & 3.6 & 7.8 \\
11.5 & 8.7 & 5.8 & 5.7 & 2.7 & 5.1 \\
\hline
\end{tabular}

\footnotetext{
${ }^{3}$ ‡Reporting standard not met (too few cases).

Note: Changes in sample size and stratification were implemented following November 2002 Youth Poll.

Source: Department of Defense Polls, JAMRS, 2001-2004 (Question: FPP9).
} 


\section{General Military Propensity}

TABLE 1-4. Youth military service propensity, by geographic region: $2001-2004^{4}$

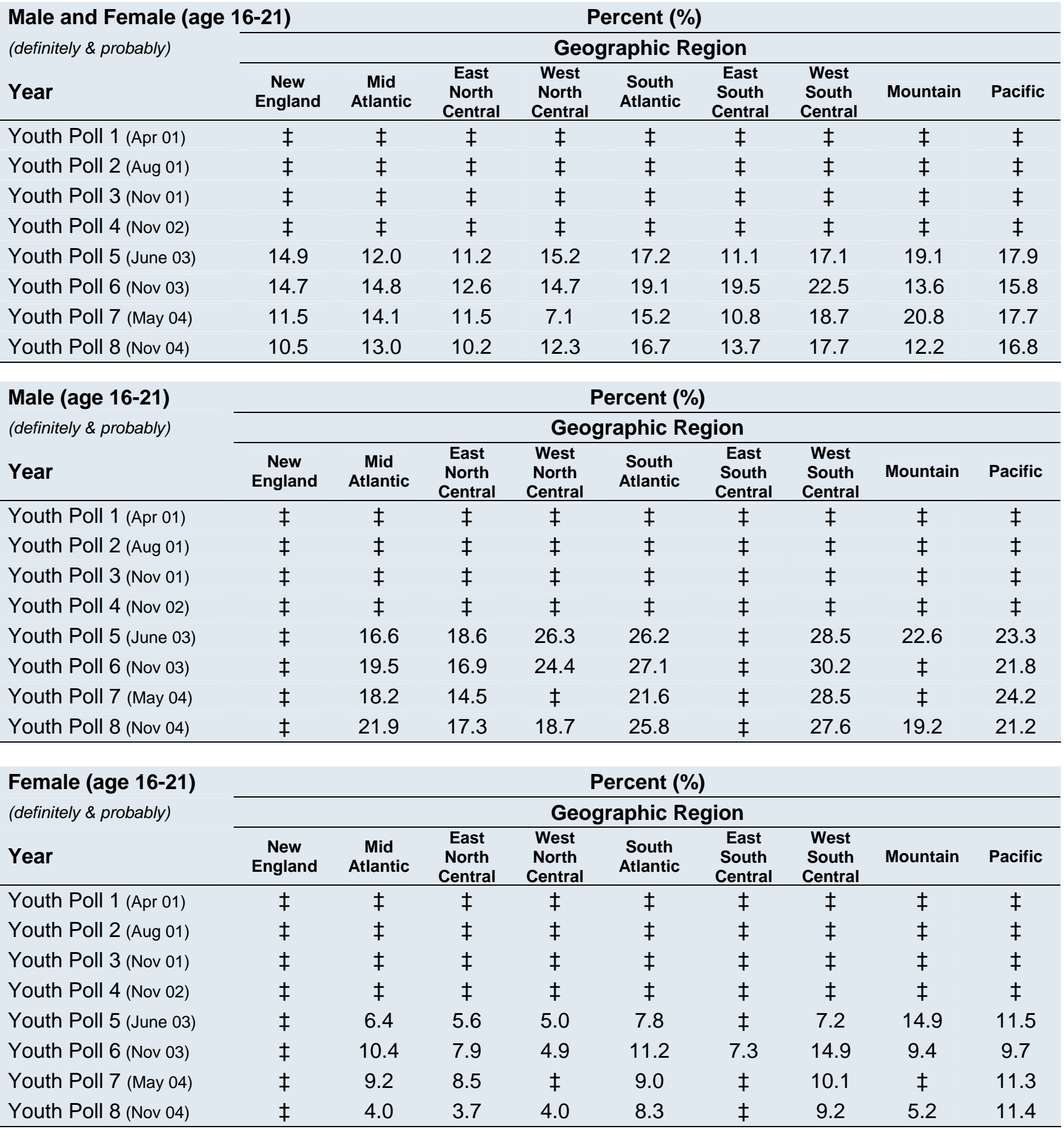

\footnotetext{
${ }^{4}$ ‡Reporting standard not met (too few cases).

Note: Changes in sample size and stratification were implemented following November 2002 Youth Poll.

Source: Department of Defense Polls, JAMRS, 2001-2004 (Question: FPP9).
} 


\section{General Military Propensity}

TABLE 1-5. Youth military service propensity, by high school grades: $2001-2004^{5}$

\begin{tabular}{|c|c|c|c|c|c|c|c|}
\hline \multirow{3}{*}{$\begin{array}{l}\text { Male and Female (age } \\
\text { (definitely \& probably) }\end{array}$} & \multicolumn{7}{|c|}{ Percent (\%) } \\
\hline & \multicolumn{7}{|c|}{ High School Grades } \\
\hline & $\begin{array}{l}\text { Mostly } \\
\text { A's }\end{array}$ & $\begin{array}{l}\text { Mostly A's } \\
\text { \& B's }\end{array}$ & $\begin{array}{l}\text { Mostly } \\
\text { B's }\end{array}$ & $\begin{array}{l}\text { Mostly B's } \\
\text { \& C's }\end{array}$ & $\begin{array}{l}\text { Mostly } \\
\text { C's }\end{array}$ & $\begin{array}{l}\text { Mostly C's } \\
\text { \& D's }\end{array}$ & $\begin{array}{l}\text { Mostly D's } \\
\text { and Lower }\end{array}$ \\
\hline Youth Poll 1 (Apr 01) & 5.2 & 9.2 & 10.2 & 15.5 & $\ddagger$ & 22.1 & $\ddagger$ \\
\hline Youth Poll 2 (Aug 01) & 6.9 & 10.5 & 8.7 & 14.7 & $\ddagger$ & 21.2 & $\ddagger$ \\
\hline Youth Poll 3 (Nov 01) & 4.1 & 10.9 & 16.2 & 21.1 & $\ddagger$ & $\ddagger$ & $\ddagger$ \\
\hline Youth Poll 4 (Nov 02) & 6.2 & 8.2 & 10.7 & 20.5 & 23.1 & $\ddagger$ & $\ddagger$ \\
\hline Youth Poll 5 (June 03) & 7.0 & 12.1 & 12.6 & 22.1 & 20.4 & 31.4 & $\ddagger$ \\
\hline Youth Poll 6 (Nov 03) & 11.4 & 14.3 & 13.1 & 21.0 & 13.8 & 35.5 & $\ddagger$ \\
\hline Youth Poll 7 (May 04) & 6.7 & 12.7 & 15.3 & 18.8 & 22.8 & 22.8 & $\ddagger$ \\
\hline Youth Poll 8 (Nov 04) & 7.5 & 12.1 & 12.2 & 17.8 & 21.1 & 32.6 & $\ddagger$ \\
\hline Male (age 16-21) & \multicolumn{7}{|c|}{ Percent (\%) } \\
\hline (definitely \& probably) & \multicolumn{7}{|c|}{ High School Grades } \\
\hline Year & $\begin{array}{l}\text { Mostly } \\
\text { A's }\end{array}$ & $\begin{array}{l}\text { Mostly A's } \\
\text { \& B's }\end{array}$ & $\begin{array}{l}\text { Mostly } \\
\text { B's }\end{array}$ & $\begin{array}{l}\text { Mostly B's } \\
\text { \& C's }\end{array}$ & $\begin{array}{l}\text { Mostly } \\
\text { C's }\end{array}$ & $\begin{array}{l}\text { Mostly C's } \\
\text { \& D's }\end{array}$ & $\begin{array}{l}\text { Mostly D's } \\
\text { and Lower }\end{array}$ \\
\hline Youth Poll 1 (Apr 01) & $\ddagger$ & 12.5 & $\ddagger$ & 20.3 & $\ddagger$ & $\ddagger$ & $\ddagger$ \\
\hline Youth Poll 2 (Aug 01) & $\ddagger$ & 10.8 & 10.9 & 19.6 & $\ddagger$ & $\ddagger$ & $\ddagger$ \\
\hline Youth Poll 3 (Nov 01) & 5.5 & 17.2 & 24.6 & 31.1 & $\ddagger$ & $\ddagger$ & $\ddagger$ \\
\hline Youth Poll 4 (Nov 02) & $\ddagger$ & 9.4 & 13.3 & 24.1 & $\ddagger$ & $\ddagger$ & $\ddagger$ \\
\hline Youth Poll 5 (June 03) & 11.7 & 18.0 & 18.9 & 29.9 & 21.2 & 37.4 & $\ddagger$ \\
\hline Youth Poll 6 (Nov 03) & 16.0 & 21.8 & 14.2 & 30.0 & 16.3 & 42.9 & $\ddagger$ \\
\hline Youth Poll 7 (May 04) & 10.1 & 18.1 & 20.4 & 23.6 & $\ddagger$ & 28.2 & $\ddagger$ \\
\hline Youth Poll 8 (Nov 04) & 11.5 & 19.4 & 17.5 & 25.7 & 25.5 & $\ddagger$ & $\ddagger$ \\
\hline Females (age 16-21) & \multicolumn{7}{|c|}{ Percent (\%) } \\
\hline (definitely \& probably) & \multicolumn{7}{|c|}{ High School Grades } \\
\hline Year & $\begin{array}{l}\text { Mostly } \\
\text { A's }\end{array}$ & $\begin{array}{l}\text { Mostly A's } \\
\text { \& B's }\end{array}$ & $\begin{array}{l}\text { Mostly } \\
\text { B's }\end{array}$ & $\begin{array}{l}\text { Mostly B's } \\
\text { \& C's }\end{array}$ & $\begin{array}{l}\text { Mostly } \\
\text { C's }\end{array}$ & $\begin{array}{l}\text { Mostly C's } \\
\text { \& D's }\end{array}$ & $\begin{array}{l}\text { Mostly D's } \\
\text { and Lower }\end{array}$ \\
\hline Youth Poll 1 (Apr 01) & 1.7 & 7.0 & $\ddagger$ & 9.9 & $\ddagger$ & $\ddagger$ & $\ddagger$ \\
\hline Youth Poll 2 (Aug 01) & 5.3 & 10.2 & 6.8 & 9.5 & $\ddagger$ & $\ddagger$ & $\ddagger$ \\
\hline Youth Poll 3 (Nov 01) & 3.2 & 6.4 & 7.4 & 9.8 & $\ddagger$ & $\ddagger$ & $\ddagger$ \\
\hline Youth Poll 4 (Nov 02) & 2.1 & 7.3 & 8.4 & 15.3 & $\ddagger$ & $\ddagger$ & $\ddagger$ \\
\hline Youth Poll 5 (June 03) & 3.8 & 7.8 & 6.8 & 10.1 & $\ddagger$ & $\ddagger$ & $\ddagger$ \\
\hline Youth Poll 6 (Nov 03) & 8.1 & 8.9 & 12.0 & 10.7 & $\ddagger$ & $\ddagger$ & $\ddagger$ \\
\hline Youth Poll 7 (May 04) & 4.1 & 9.0 & 9.5 & 12.8 & $\ddagger$ & $\ddagger$ & $\ddagger$ \\
\hline Youth Poll 8 (Nov 04) & 5.0 & 5.8 & 6.8 & 7.1 & $\ddagger$ & $\ddagger$ & $\ddagger$ \\
\hline
\end{tabular}

${ }^{5}$ ‡Reporting standard not met (too few cases).

Note: Changes in sample size and stratification were implemented following November 2002 Youth Poll.

Source: Department of Defense Polls, JAMRS, 2001-2004 (Question: FPP9). 


\section{Composite Active Duty Propensity}

TABLE 2-1. Youth composite active duty propensity: $2001-2004^{6}$

Male and Female (age 16-21)

Percent (\%)

\begin{tabular}{lcccc}
\cline { 2 - 4 } Year & Definitely & Probably & Probably Not & Definitely Not \\
\hline Youth Poll 1 (Apr 01) & 4.0 & 13.8 & 24.6 & 57.7 \\
Youth Poll 2 (Aug 01) & 4.2 & 13.1 & 29.4 & 53.3 \\
Youth Poll 3 (Nov 01) & 5.0 & 16.9 & 30.3 & 47.8 \\
Youth Poll 4 (Nov 02) & 4.4 & 15.4 & 27.5 & 52.7 \\
Youth Poll 5 (June 03) & 5.8 & 15.6 & 28.6 & 49.9 \\
Youth Poll 6 (Nov 03) & 5.5 & 18.7 & 26.4 & 49.5 \\
Youth Poll 7 (May 04) & 5.7 & 17.0 & 29.3 & 48.0 \\
Youth Poll 8 (Nov 04) & 5.5 & 15.8 & 28.3 & 50.4 \\
\hline
\end{tabular}

Male (age 16-21)

Percent (\%)

\begin{tabular}{lcccc}
\cline { 2 - 4 } Year & Definitely & Probably & Probably Not & Definitely Not \\
\hline Youth Poll 1 (Apr 01) & 6.5 & 18.9 & 30.6 & 44.1 \\
Youth Poll 2 (Aug 01) & 5.5 & 15.7 & 36.1 & 42.6 \\
Youth Poll 3 (Nov 01) & 8.3 & 24.0 & 34.7 & 33.0 \\
Youth Poll 4 (Nov 02) & 6.0 & 21.0 & 30.2 & 42.8 \\
Youth Poll 5 (June 03) & 8.4 & 20.9 & 33.4 & 37.2 \\
Youth Poll 6 (Nov 03) & 8.6 & 23.7 & 31.6 & 36.0 \\
Youth Poll 7 (May 04) & 8.7 & 21.3 & 34.1 & 35.9 \\
Youth Poll 8 (Nov 04) & 8.3 & 22.4 & 31.6 & 37.6 \\
\hline
\end{tabular}

Female (age 16-21)

Percent (\%)

\begin{tabular}{lcccc}
\cline { 2 - 4 } Year & Definitely & Probably & Probably Not & Definitely Not \\
\hline Youth Poll 1 (Apr 01) & 1.7 & 9.1 & 19.0 & 70.2 \\
Youth Poll 2 (Aug 01) & 3.0 & 10.6 & 23.0 & 63.4 \\
Youth Poll 3 (Nov 01) & 2.0 & 10.1 & 26.1 & 61.7 \\
Youth Poll 4 (Nov 02) & 3.0 & 9.9 & 24.9 & 62.2 \\
Youth Poll 5 (June 03) & 3.0 & 10.2 & 23.8 & 63.0 \\
Youth Poll 6 (Nov 03) & 2.3 & 13.6 & 21.1 & 63.0 \\
Youth Poll 7 (May 04) & 2.6 & 12.5 & 24.3 & 60.5 \\
Youth Poll 8 (Nov 04) & 2.6 & 9.0 & 24.9 & 63.4 \\
\hline
\end{tabular}

${ }^{6}$ ‡Reporting standard not met (too few cases).

Note: Changes in sample size and stratification were implemented following November 2002 Youth Poll.

Source: Department of Defense Polls, JAMRS, 2001-2004 (Question: Composite of FPP10B, FPP10C, FPP10D, FPP10E). 


\section{Composite Active Duty Propensity}

TABLE 2-2. Youth composite active duty propensity, by race/ethnicity: $2001-2004^{7}$

\begin{tabular}{|c|c|c|c|c|}
\hline \multirow{3}{*}{$\begin{array}{l}\text { Male and Female (age 16-21) } \\
\text { (definitely \& probably) } \\
\text { Year }\end{array}$} & \multicolumn{4}{|c|}{ Percent (\%) } \\
\hline & \multicolumn{4}{|c|}{ Race/ethnicity } \\
\hline & Total & White & Black & Hispanic \\
\hline Youth Poll 1 (Apr 01) & 17.8 & 13.9 & 27.8 & 24.3 \\
\hline Youth Poll 2 (Aug 01) & 17.3 & 11.5 & 28.2 & 27.2 \\
\hline Youth Poll 3 (Nov 01) & 21.9 & 18.6 & 22.7 & 35.1 \\
\hline Youth Poll 4 (Nov 02) & 19.8 & 15.3 & 25.8 & 29.9 \\
\hline Youth Poll 5 (June 03) & 21.4 & 16.9 & 26.2 & 34.3 \\
\hline Youth Poll 6 (Nov 03) & 24.1 & 18.6 & 32.3 & 35.1 \\
\hline Youth Poll 7 (May 04) & 22.6 & 17.4 & 27.7 & 38.5 \\
\hline Youth Poll 8 (Nov 04) & 21.3 & 17.2 & 21.2 & 33.4 \\
\hline Male (age 16-21) & \multicolumn{4}{|c|}{ Percent (\%) } \\
\hline (definitely \& probably) & \multicolumn{4}{|c|}{ Race/ethnicity } \\
\hline Year & Total & White & Black & Hispanic \\
\hline Youth Poll 1 (Apr 01) & 25.3 & 21.4 & 36.0 & 33.5 \\
\hline Youth Poll 2 (Aug 01) & 21.2 & 15.2 & 37.4 & 31.4 \\
\hline Youth Poll 3 (Nov 01) & 32.3 & 28.5 & 33.5 & 46.7 \\
\hline Youth Poll 4 (Nov 02) & 27.0 & 22.0 & 32.0 & 41.0 \\
\hline Youth Poll 5 (June 03) & 29.3 & 24.0 & 34.4 & 45.7 \\
\hline Youth Poll 6 (Nov 03) & 32.3 & 26.7 & 39.1 & 45.2 \\
\hline Youth Poll 7 (May 04) & 30.0 & 24.1 & 36.2 & 46.2 \\
\hline Youth Poll 8 (Nov 04) & 30.7 & 27.0 & 26.3 & 43.3 \\
\hline Female (age 16-21) & \multicolumn{4}{|c|}{ Percent (\%) } \\
\hline (definitely \& probably) & \multicolumn{4}{|c|}{ Race/ethnicity } \\
\hline Year & Total & White & Black & Hispanic \\
\hline Youth Poll 1 (Apr 01) & 10.8 & 6.5 & 22.2 & 16.4 \\
\hline Youth Poll 2 (Aug 01) & 13.6 & 7.7 & 22.5 & 22.7 \\
\hline Youth Poll 3 (Nov 01) & 12.2 & 8.8 & 16.4 & 22.7 \\
\hline Youth Poll 4 (Nov 02) & 12.9 & 8.6 & 19.7 & 21.2 \\
\hline Youth Poll 5 (June 03) & 13.2 & 9.3 & 19.5 & 22.4 \\
\hline Youth Poll 6 (Nov 03) & 15.9 & 10.0 & 26.6 & 25.3 \\
\hline Youth Poll 7 (May 04) & 15.1 & 10.1 & 20.3 & 30.7 \\
\hline Youth Poll 8 (Nov 04) & 11.6 & 6.2 & 17.5 & 23.3 \\
\hline
\end{tabular}

\footnotetext{
${ }^{7}$ Due to relatively small sample sizes, American Indian, Alaska Native, Asian, and Pacific Islander are included in the total but are not shown separately. Black includes African American, and Hispanic includes Latino. Race categories exclude Hispanic origin unless specified otherwise.

‡Reporting standard not met (too few cases).

Note: Changes in sample size and stratification were implemented following November 2002 Youth Poll.

Source: Department of Defense Polls, JAMRS, 2001-2004 (Question: Composite of FPP10B, FPP10C, FPP10D, FPP10E).
} 


\section{Composite Active Duty Propensity}

TABLE 2-3. Youth composite active duty propensity, by age: $2001-2004^{8}$

\begin{tabular}{lcccccc} 
Male and Female (age 16-21) & \multicolumn{7}{c}{ Percent (\%) } \\
\cline { 2 - 8 } (definitely \& probably) & \multicolumn{7}{c}{ Age } \\
\cline { 2 - 7 } Year & $\mathbf{1 6}$ & $\mathbf{1 7}$ & $\mathbf{1 8}$ & $\mathbf{1 9}$ & $\mathbf{2 0}$ & $\mathbf{2 1}$ \\
\hline Youth Poll 1 (Apr 01) & 28.2 & 21.6 & 16.8 & 15.3 & 12.1 & 12.3 \\
Youth Poll 2 (Aug 01) & 29.3 & 19.8 & 20.7 & 11.7 & 14.1 & 7.6 \\
Youth Poll 3 (Nov 01) & 30.5 & 26.6 & 20.8 & 16.5 & 20.6 & 16.2 \\
Youth Poll 4 (Nov 02) & 28.3 & 24.4 & 19.2 & 17.1 & 18.5 & 10.7 \\
Youth Poll 5 (June 03) & 31.7 & 25.0 & 23.2 & 18.4 & 16.1 & 12.6 \\
Youth Poll 6 (Nov 03) & 36.9 & 27.0 & 24.4 & 16.6 & 19.3 & 18.5 \\
Youth Poll 7 (May 04) & 31.8 & 28.8 & 21.6 & 19.7 & 16.4 & 15.8 \\
Youth Poll 8 (Nov 04) & 31.0 & 23.2 & 21.3 & 16.8 & 18.0 & 15.9 \\
\hline
\end{tabular}

\begin{tabular}{lcccccc} 
Male (age 16-21) & \multicolumn{7}{c}{ Percent (\%) } \\
\cline { 2 - 7 } $\begin{array}{l}\text { (definitely \& probably) } \\
\text { Year }\end{array}$ & $\mathbf{1 6}$ & $\mathbf{1 7}$ & $\mathbf{1 8}$ & $\mathbf{1 9}$ & $\mathbf{2 0}$ & $\mathbf{2 1}$ \\
\hline & 35.2 & 29.1 & 25.6 & 23.8 & 13.8 & 22.7 \\
\hline Youth Poll 1 (Apr 01) & 32.7 & 25.4 & 22.8 & 16.1 & 21.0 & 4.8 \\
Youth Poll 2 (Aug 01) & 41.8 & 37.8 & 29.1 & 25.7 & 29.5 & 28.8 \\
Youth Poll 3 (Nov 01) & 31.3 & 35.7 & 28.3 & 24.7 & 27.0 & 12.1 \\
Youth Poll 4 (Nov 02) & 39.3 & 31.0 & 32.7 & 29.3 & 23.8 & 18.5 \\
Youth Poll 5 (June 03) & 46.0 & 34.8 & 32.2 & 24.6 & 28.6 & 25.2 \\
Youth Poll 6 (Nov 03) & 41.5 & 36.6 & 28.3 & 24.7 & 25.8 & 21.2 \\
Youth Poll 7 (May 04) & 42.3 & 30.5 & 32.8 & 27.1 & 28.0 & 22.0 \\
Youth Poll 8 (Nov 04) & & & & & & \\
\hline
\end{tabular}

\begin{tabular}{lcccccc} 
Female (age 16-21) & \multicolumn{7}{c}{ Percent (\%) } \\
\cline { 2 - 7 } (definitely \& probably) & $\mathbf{7 9}$ & $\mathbf{1 7}$ & $\mathbf{1 9}$ & $\mathbf{2 0}$ & $\mathbf{2 1}$ \\
\cline { 2 - 7 } Year & 21.4 & 12.8 & 8.9 & 6.5 & 10.7 & 5.3 \\
\hline Youth Poll 1 (Apr 01) & 25.0 & 13.8 & 18.8 & 7.7 & 8.4 & 9.7 \\
Youth Poll 2 (Aug 01) & 19.7 & 14.3 & 12.3 & 9.1 & 13.4 & 4.2 \\
Youth Poll 3 (Nov 01) & 24.9 & 13.7 & 9.8 & 10.4 & 9.5 & 9.6 \\
Youth Poll 4 (Nov 02) & 23.9 & 18.6 & 13.4 & 7.5 & 7.7 & 7.1 \\
Youth Poll 5 (June 03) & 27.8 & 18.4 & 16.8 & 8.5 & 10.5 & 11.9 \\
Youth Poll 6 (Nov 03) & 21.9 & 21.4 & 14.0 & 14.5 & 6.7 & 10.4 \\
Youth Poll 7 (May 04) & 19.1 & 15.7 & 9.5 & 6.5 & 7.8 & 9.8 \\
Youth Poll 8 (Nov 04) & & & & & 7.5 \\
\hline
\end{tabular}

\footnotetext{
${ }^{8}$ ‡Reporting standard not met (too few cases). Note: Changes in sample size and stratification were implemented following November 2002 Youth Poll. Source: Department of Defense Polls, JAMRS, 2001-2004 (Question: Composite of FPP10B, FPP10C, FPP10D, FPP10E).
} 


\section{Composite Active Duty Propensity}

TABLE 2-4. Youth composite active duty propensity, by geographic region: $2001-2004^{9}$

\begin{tabular}{|c|c|c|c|c|c|c|c|c|c|}
\hline \multirow{3}{*}{$\begin{array}{l}\text { Male and Female (age } \\
\text { (definitely \& probably) } \\
\text { Year }\end{array}$} & \multicolumn{9}{|c|}{ Percent (\%) } \\
\hline & \multicolumn{9}{|c|}{ Geographic Region } \\
\hline & $\begin{array}{c}\text { New } \\
\text { England }\end{array}$ & $\begin{array}{c}\text { Mid } \\
\text { Atlantic }\end{array}$ & $\begin{array}{c}\text { East } \\
\text { North } \\
\text { Central }\end{array}$ & $\begin{array}{c}\text { West } \\
\text { North } \\
\text { Central }\end{array}$ & $\begin{array}{c}\text { South } \\
\text { Atlantic }\end{array}$ & $\begin{array}{c}\text { East } \\
\text { South } \\
\text { Central }\end{array}$ & $\begin{array}{c}\text { West } \\
\text { South } \\
\text { Central }\end{array}$ & Mountain & Pacific \\
\hline Youth Poll 1 (Apr 01) & $\ddagger$ & $\ddagger$ & $\ddagger$ & $\ddagger$ & $\ddagger$ & $\ddagger$ & $\ddagger$ & $\ddagger$ & $\ddagger$ \\
\hline Youth Poll 2 (Aug 01) & $\ddagger$ & $\ddagger$ & $\ddagger$ & $\ddagger$ & $\ddagger$ & $\ddagger$ & $\ddagger$ & $\ddagger$ & $\ddagger$ \\
\hline Youth Poll 3 (Nov 01) & $\ddagger$ & $\ddagger$ & $\ddagger$ & $\ddagger$ & $\ddagger$ & $\ddagger$ & $\ddagger$ & $\ddagger$ & $\ddagger$ \\
\hline Youth Poll 4 (Nov 02) & $\ddagger$ & $\ddagger$ & $\ddagger$ & $\ddagger$ & $\ddagger$ & $\ddagger$ & $\ddagger$ & $\ddagger$ & $\ddagger$ \\
\hline Youth Poll 5 (June 03) & 20.8 & 16.2 & 15.9 & 20.3 & 23.9 & 20.0 & 23.8 & 23.6 & 25.9 \\
\hline Youth Poll 6 (Nov 03) & 25.1 & 20.9 & 19.0 & 18.9 & 27.0 & 28.9 & 32.3 & 20.4 & 24.8 \\
\hline Youth Poll 7 (May 04) & 20.3 & 20.2 & 19.8 & 11.7 & 20.5 & 16.1 & 27.6 & 30.7 & 29.1 \\
\hline Youth Poll 8 (Nov 04) & 17.4 & 19.4 & 15.8 & 17.5 & 24.5 & 19.3 & 28.1 & 18.5 & 24.3 \\
\hline Male (age 16-21) & \multicolumn{9}{|c|}{ Percent (\%) } \\
\hline (definitely \& probably) & \multicolumn{9}{|c|}{ Geographic Region } \\
\hline Year & $\begin{array}{c}\text { New } \\
\text { England }\end{array}$ & $\begin{array}{c}\text { Mid } \\
\text { Atlantic } \\
\end{array}$ & $\begin{array}{c}\text { East } \\
\text { North } \\
\text { Central }\end{array}$ & $\begin{array}{c}\text { West } \\
\text { North } \\
\text { Central } \\
\end{array}$ & $\begin{array}{c}\text { South } \\
\text { Atlantic }\end{array}$ & $\begin{array}{c}\text { East } \\
\text { South } \\
\text { Central }\end{array}$ & $\begin{array}{c}\text { West } \\
\text { South } \\
\text { Central }\end{array}$ & Mountain & Pacific \\
\hline Youth Poll 1 (Apr 01) & $\ddagger$ & $\ddagger$ & $\ddagger$ & $\ddagger$ & $\ddagger$ & $\ddagger$ & $\ddagger$ & $\ddagger$ & $\ddagger$ \\
\hline Youth Poll 2 (Aug 01) & $\ddagger$ & $\ddagger$ & $\ddagger$ & $\ddagger$ & $\ddagger$ & $\ddagger$ & $\ddagger$ & $\ddagger$ & $\ddagger$ \\
\hline Youth Poll 3 (Nov 01) & $\ddagger$ & $\ddagger$ & $\ddagger$ & $\ddagger$ & $\ddagger$ & $\ddagger$ & $\ddagger$ & $\ddagger$ & $\ddagger$ \\
\hline Youth Poll 4 (Nov 02) & $\ddagger$ & $\ddagger$ & $\ddagger$ & $\ddagger$ & $\ddagger$ & $\ddagger$ & $\ddagger$ & $\ddagger$ & $\ddagger$ \\
\hline Youth Poll 5 (June 03) & $\ddagger$ & 19.5 & 24.4 & 33.1 & 35.3 & $\ddagger$ & 34.3 & 28.7 & 32.8 \\
\hline Youth Poll 6 (Nov 03) & $\ddagger$ & 25.4 & 24.8 & 30.2 & 35.7 & $\ddagger$ & 42.1 & $\ddagger$ & 33.1 \\
\hline Youth Poll 7 (May 04) & $\ddagger$ & 26.4 & 24.9 & $\ddagger$ & 29.2 & $\ddagger$ & 40.2 & $\ddagger$ & 39.1 \\
\hline Youth Poll 8 (Nov 04) & $\ddagger$ & 31.5 & 23.8 & 26.5 & 35.2 & $\ddagger$ & 38.8 & 26.0 & 32.6 \\
\hline Female (age 16-21) & \multicolumn{9}{|c|}{ Percent (\%) } \\
\hline (definitely \& probably) & \multicolumn{9}{|c|}{ Geographic Region } \\
\hline Year & $\begin{array}{c}\text { New } \\
\text { England }\end{array}$ & $\begin{array}{c}\text { Mid } \\
\text { Atlantic } \\
\end{array}$ & $\begin{array}{c}\text { East } \\
\text { North } \\
\text { Central } \\
\end{array}$ & $\begin{array}{c}\text { West } \\
\text { North } \\
\text { Central } \\
\end{array}$ & $\begin{array}{c}\text { South } \\
\text { Atlantic } \\
\end{array}$ & $\begin{array}{c}\text { East } \\
\text { South } \\
\text { Central }\end{array}$ & $\begin{array}{c}\text { West } \\
\text { South } \\
\text { Central }\end{array}$ & Mountain & Pacific \\
\hline Youth Poll 1 (Apr 01) & $\ddagger$ & $\ddagger$ & $\ddagger$ & $\ddagger$ & $\ddagger$ & $\ddagger$ & $\ddagger$ & $\ddagger$ & $\ddagger$ \\
\hline Youth Poll 2 (Aug 01) & $\ddagger$ & $\ddagger$ & $\ddagger$ & $\ddagger$ & $\ddagger$ & $\ddagger$ & $\ddagger$ & $\ddagger$ & $\ddagger$ \\
\hline Youth Poll 3 (Nov 01) & $\ddagger$ & $\ddagger$ & $\ddagger$ & $\ddagger$ & $\ddagger$ & $\ddagger$ & $\ddagger$ & $\ddagger$ & $\ddagger$ \\
\hline Youth Poll 4 (Nov 02) & $\ddagger$ & $\ddagger$ & $\ddagger$ & $\ddagger$ & $\ddagger$ & $\ddagger$ & $\ddagger$ & $\ddagger$ & $\ddagger$ \\
\hline Youth Poll 5 (June 03) & $\ddagger$ & 12.0 & 9.4 & 8.5 & 12.1 & $\ddagger$ & 14.6 & 17.4 & 17.7 \\
\hline Youth Poll 6 (Nov 03) & $\ddagger$ & 16.7 & 12.6 & 7.4 & 18.5 & 14.4 & 22.7 & 15.6 & 16.2 \\
\hline Youth Poll 7 (May 04) & $\ddagger$ & 12.6 & 14.7 & $\ddagger$ & 12.1 & $\ddagger$ & 16.6 & $\ddagger$ & 19.4 \\
\hline Youth Poll 8 (Nov 04) & $\ddagger$ & 7.1 & 8.6 & 5.9 & 14.5 & $\ddagger$ & 18.8 & 11.0 & 14.4 \\
\hline
\end{tabular}

${ }^{9}$ ‡Reporting standard not met (too few cases).

Note: Changes in sample size and stratification were implemented following November 2002 Youth Poll.

Source: Department of Defense Polls, JAMRS, 2001-2004 (Question: Composite of FPP10B, FPP10C, FPP10D, FPP10E).

Page A-9 DoD May 2004 Youth Poll 


\section{Composite Active Duty Propensity}

TABLE 2-5. Youth composite active duty propensity, by high school grades: $2001-2004^{10}$

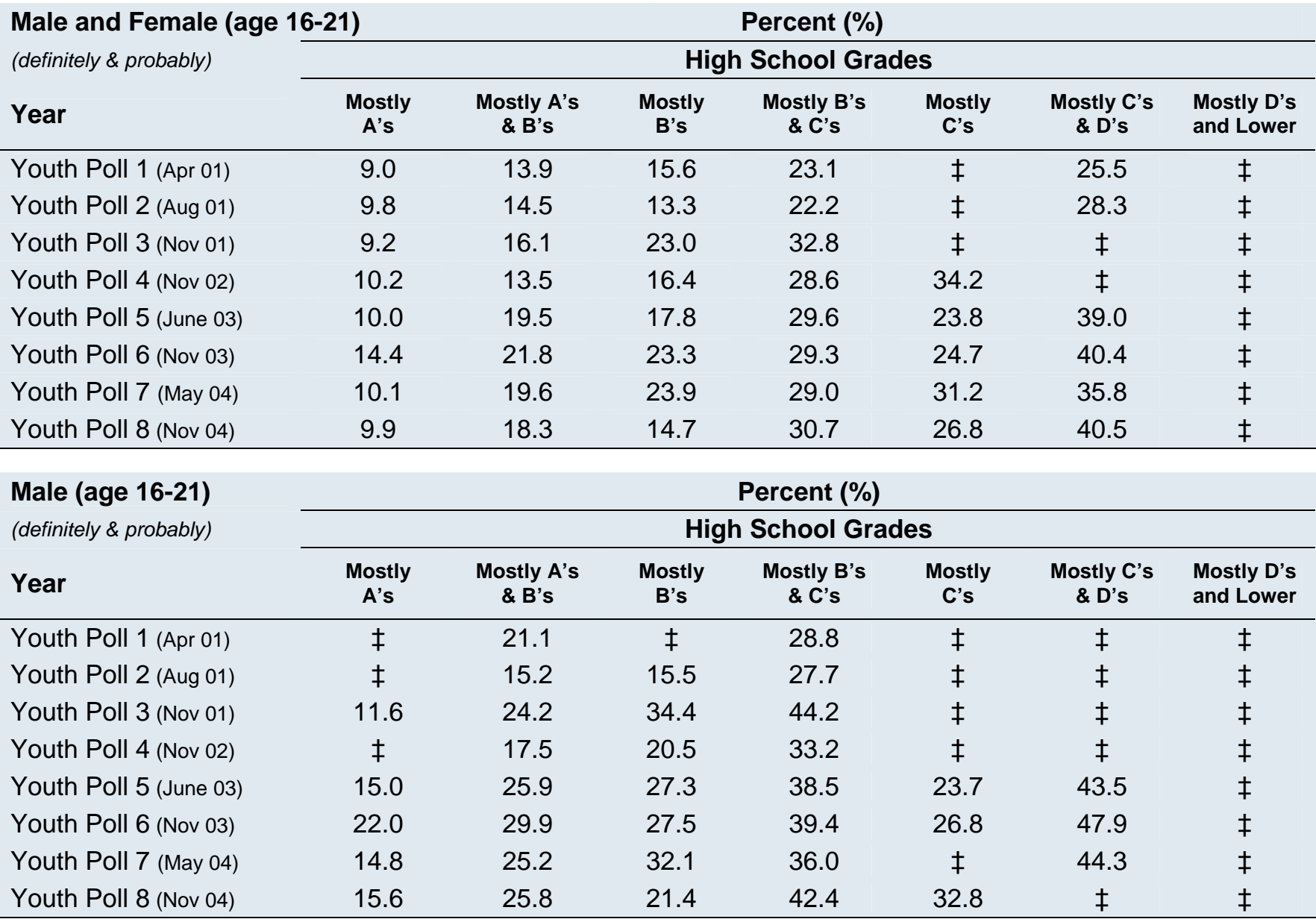

Females (age 16-21)

(definitely \& probably)

Year

Youth Poll 1 (Apr 01)

Youth Poll 2 (Aug 01)

Youth Poll 3 (Nov 01)

Youth Poll 4 (Nov 02)

Youth Poll 5 (June 03)

Youth Poll 6 (Nov 03)

Youth Poll 7 (May 04)

Youth Poll 8 (Nov 04)

Percent (\%)

High School Grades

\begin{tabular}{ccccccc}
$\begin{array}{c}\text { Mostly } \\
\text { A's }\end{array}$ & $\begin{array}{c}\text { Mostly A's } \\
\text { \& B's }\end{array}$ & $\begin{array}{c}\text { Mostly } \\
\text { B's }\end{array}$ & $\begin{array}{c}\text { Mostly B's } \\
\text { \& C's }\end{array}$ & $\begin{array}{c}\text { Mostly } \\
\text { C's }\end{array}$ & $\begin{array}{c}\text { Mostly C's } \\
\text { \& D's }\end{array}$ & $\begin{array}{c}\text { Mostly D's } \\
\text { and Lower }\end{array}$ \\
\hline 6.5 & 9.2 & $\ddagger$ & 16.4 & $\ddagger$ & $\ddagger$ & $\ddagger$ \\
8.9 & 13.9 & 11.4 & 16.3 & $\ddagger$ & $\ddagger$ & $\ddagger$ \\
7.7 & 10.3 & 11.4 & 19.9 & $\ddagger$ & $\ddagger$ & $\ddagger$ \\
3.8 & 10.7 & 12.9 & 21.7 & $\ddagger$ & $\ddagger$ & $\ddagger$ \\
6.6 & 14.6 & 9.1 & 15.9 & $\ddagger$ & $\ddagger$ & $\ddagger$ \\
8.9 & 15.9 & 19.1 & 17.7 & $\ddagger$ & $\ddagger$ & $\ddagger$ \\
6.6 & 15.9 & 14.3 & 20.0 & $\ddagger$ & $\ddagger$ & $\ddagger$ \\
6.5 & 11.9 & 8.1 & 14.8 & $\ddagger$ & $\ddagger$ & $\ddagger$
\end{tabular}

${ }^{10} \ddagger$ Reporting standard not met (too few cases).

Note: Changes in sample size and stratification were implemented following November 2002 Youth Poll.

Source: Department of Defense Polls, JAMRS, 2001-2004 (Question: Composite of FPP10B, FPP10C, FPP10D, FPP10E). 


\section{Army Active Duty Propensity}

TABLE 3-1. Youth Army active duty propensity: $2001-2004^{11}$

Male and Female (age 16-21)

Percent (\%)

\begin{tabular}{lcccc}
\cline { 2 - 4 } Year & Definitely & Probably & Probably Not & Definitely Not \\
\hline Youth Poll 1 (Apr 01) & .9 & 5.6 & 23.3 & 70.0 \\
Youth Poll 2 (Aug 01) & 1.1 & 6.3 & 25.9 & 66.6 \\
Youth Poll 3 (Nov 01) & 2.2 & 8.0 & 30.0 & 59.6 \\
Youth Poll 4 (Nov 02) & 1.0 & 7.9 & 27.1 & 63.8 \\
Youth Poll 5 (June 03) & 1.9 & 7.8 & 29.0 & 61.2 \\
Youth Poll 6 (Nov 03) & 1.8 & 9.2 & 26.5 & 62.6 \\
Youth Poll 7 (May 04) & 1.8 & 8.3 & 29.7 & 60.1 \\
Youth Poll 8 (Nov 04) & 1.3 & 8.3 & 28.0 & 62.4 \\
\hline
\end{tabular}

Male (age 16-21)

Percent (\%)

\begin{tabular}{lcccc}
\cline { 2 - 4 } Year & Definitely & Probably & Probably Not & Definitely Not \\
\hline Youth Poll 1 (Apr 01) & 1.6 & 8.2 & 30.0 & 60.2 \\
Youth Poll 2 (Aug 01) & 1.5 & 8.0 & 32.9 & 57.6 \\
Youth Poll 3 (Nov 01) & 3.7 & 12.2 & 36.7 & 47.1 \\
Youth Poll 4 (Nov 02) & 1.5 & 11.5 & 32.3 & 54.5 \\
Youth Poll 5 (June 03) & 2.8 & 11.1 & 35.4 & 50.5 \\
Youth Poll 6 (Nov 03) & 2.8 & 12.5 & 33.4 & 51.3 \\
Youth Poll 7 (May 04) & 2.8 & 10.4 & 36.2 & 50.5 \\
Youth Poll 8 (Nov 04) & 1.9 & 12.5 & 34.6 & 50.9 \\
\hline
\end{tabular}

Female (age 16-21)

Percent (\%)

\begin{tabular}{lcccc}
\cline { 2 - 4 } Year & Definitely & Probably & Probably Not & Definitely Not \\
\hline Youth Poll 1 (Apr 01) & .3 & 3.2 & 17.1 & 79.1 \\
Youth Poll 2 (Aug 01) & .7 & 4.7 & 19.4 & 75.0 \\
Youth Poll 3 (Nov 01) & .7 & 4.1 & 23.8 & 71.3 \\
Youth Poll 4 (Nov 02) & .6 & 4.4 & 22.1 & 72.8 \\
Youth Poll 5 (June 03) & 1.1 & 4.5 & 22.4 & 72.1 \\
Youth Poll 6 (Nov 03) & .7 & 5.8 & 19.5 & 74.0 \\
Youth Poll 7 (May 04) & .8 & 6.2 & 23.0 & 69.9 \\
Youth Poll 8 (Nov 04) & .7 & 4.0 & 21.2 & 74.1 \\
\hline
\end{tabular}

${ }^{11} \ddagger$ Reporting standard not met (too few cases).

Note: Changes in sample size and stratification were implemented following November 2002 Youth Poll.

Source: Department of Defense Polls, JAMRS, 2001-2004 (Question: FPP10B). 


\section{Army Active Duty Propensity}

TABLE 3-2. Youth Army active duty propensity, by race/ethnicity: $2001-2004^{12}$

\begin{tabular}{|c|c|c|c|c|}
\hline \multirow{3}{*}{$\begin{array}{l}\text { Male and Female (age 16-21) } \\
\text { (definitely \& probably) } \\
\text { Year }\end{array}$} & \multicolumn{4}{|c|}{ Percent (\%) } \\
\hline & \multicolumn{4}{|c|}{ Race/ethnicity } \\
\hline & Total & White & Black & Hispanic \\
\hline Youth Poll 1 (Apr 01) & 6.5 & 5.1 & 10.5 & 9.2 \\
\hline Youth Poll 2 (Aug 01) & 7.4 & 4.4 & 11.0 & 15.3 \\
\hline Youth Poll 3 (Nov 01) & 10.2 & 7.8 & 10.6 & 19.3 \\
\hline Youth Poll 4 (Nov 02) & 9.0 & 5.8 & 12.7 & 15.8 \\
\hline Youth Poll 5 (June 03) & 9.8 & 7.8 & 9.5 & 17.3 \\
\hline Youth Poll 6 (Nov 03) & 10.9 & 7.9 & 13.8 & 18.6 \\
\hline Youth Poll 7 (May 04) & 10.1 & 8.0 & 10.7 & 18.2 \\
\hline Youth Poll 8 (Nov 04) & 9.6 & 7.6 & 7.5 & 17.7 \\
\hline Male (age 16-21) & \multicolumn{4}{|c|}{ Percent (\%) } \\
\hline (definitely \& probably) & \multicolumn{4}{|c|}{ Race/ethnicity } \\
\hline Year & Total & White & Black & Hispanic \\
\hline Youth Poll 1 (Apr 01) & 9.8 & 8.2 & 17.0 & 11.1 \\
\hline Youth Poll 2 (Aug 01) & 9.5 & 5.7 & 14.3 & 20.3 \\
\hline Youth Poll 3 (Nov 01) & 15.9 & 13.3 & 16.5 & 24.8 \\
\hline Youth Poll 4 (Nov 02) & 13.0 & 10.0 & 15.6 & 20.0 \\
\hline Youth Poll 5 (June 03) & 13.9 & 11.6 & 10.9 & 24.6 \\
\hline Youth Poll 6 (Nov 03) & 15.3 & 12.3 & 18.2 & 24.2 \\
\hline Youth Poll 7 (May 04) & 13.1 & 11.1 & 13.2 & 21.5 \\
\hline Youth Poll 8 (Nov 04) & 14.4 & 12.4 & 9.9 & 22.6 \\
\hline Female (age $16-21$ ) & \multicolumn{4}{|c|}{ Percent (\%) } \\
\hline (definitely \& probably) & \multicolumn{4}{|c|}{ Race/ethnicity } \\
\hline Year & Total & White & Black & Hispanic \\
\hline Youth Poll 1 (Apr 01) & 3.6 & 2.0 & 6.0 & 7.5 \\
\hline Youth Poll 2 (Aug 01) & 5.4 & 3.1 & 8.9 & 10.1 \\
\hline Youth Poll 3 (Nov 01) & 4.9 & 2.3 & 7.1 & 13.4 \\
\hline Youth Poll 4 (Nov 02) & 5.1 & 1.7 & 9.9 & 12.5 \\
\hline Youth Poll 5 (June 03) & 5.5 & 3.8 & 8.4 & 9.6 \\
\hline Youth Poll 6 (Nov 03) & 6.5 & 3.3 & 10.2 & 13.2 \\
\hline Youth Poll 7 (May 04) & 7.0 & 4.6 & 8.4 & 14.8 \\
\hline Youth Poll 8 (Nov 04) & 4.7 & 2.2 & 5.9 & 12.7 \\
\hline
\end{tabular}

\footnotetext{
${ }^{12}$ Due to relatively small sample sizes, American Indian, Alaska Native, Asian, and Pacific Islander are included in the total but are not shown separately. Black includes African American, and Hispanic includes Latino. Race categories exclude Hispanic origin unless specified otherwise.

‡Reporting standard not met (too few cases).

Note: Changes in sample size and stratification were implemented following November 2002 Youth Poll. Source: Department of Defense Polls, JAMRS, 2001-2004 (Question: FPP10B).
} 


\section{Army Active Duty Propensity}

JAMRS

TABLE 3-3. Youth Army active duty propensity, by age: $2001-2004^{13}$

\begin{tabular}{lcccccc} 
Male and Female (age 16-21) & \multicolumn{7}{c}{ Percent (\%) } \\
\cline { 2 - 8 } (definitely \& probably) & \multicolumn{7}{c}{ Age } \\
\cline { 2 - 7 } Year & $\mathbf{1 6}$ & $\mathbf{1 7}$ & $\mathbf{1 8}$ & $\mathbf{1 9}$ & $\mathbf{2 0}$ & $\mathbf{2 1}$ \\
\hline Youth Poll 1 (Apr 01) & 9.5 & 7.7 & 5.5 & 7.1 & 5.5 & 3.8 \\
Youth Poll 2 (Aug 01) & 12.4 & 10.5 & 9.3 & 3.1 & 7.2 & 1.6 \\
Youth Poll 3 (Nov 01) & 13.7 & 12.1 & 9.2 & 7.7 & 8.8 & 9.9 \\
Youth Poll 4 (Nov 02) & 13.9 & 10.7 & 7.9 & 8.1 & 9.5 & 3.1 \\
Youth Poll 5 (June 03) & 14.3 & 11.5 & 10.4 & 8.0 & 7.7 & 6.1 \\
Youth Poll 6 (Nov 03) & 17.3 & 12.8 & 11.1 & 6.3 & 9.0 & 8.0 \\
Youth Poll 7 (May 04) & 15.4 & 14.6 & 7.2 & 7.6 & 7.6 & 7.0 \\
Youth Poll 8 (Nov 04) & 12.7 & 8.6 & 11.1 & 7.4 & 9.8 & 7.6 \\
\hline
\end{tabular}

\section{Male (age 16-21)}

(definitely \& probably)

Year

Youth Poll 1 (Apr 01)

Youth Poll 2 (Aug 01)

Youth Poll 3 (Nov 01)

Youth Poll 4 (Nov 02)

Youth Poll 5 (June 03)

Youth Poll 6 (Nov 03)

Youth Poll 7 (May 04)

Youth Poll 8 (Nov 04)

\begin{tabular}{cccccc}
\multicolumn{7}{c}{ Percent (\%) } \\
\hline \multicolumn{7}{c}{ Age } \\
\hline 16 & $\mathbf{1 7}$ & $\mathbf{1 8}$ & $\mathbf{1 9}$ & $\mathbf{2 0}$ & $\mathbf{2 1}$ \\
\hline 10.8 & 9.4 & 9.6 & 11.0 & 8.1 & 9.5 \\
15.7 & 12.5 & 11.9 & 4.0 & 10.7 & 0.0 \\
19.7 & 17.8 & 16.0 & 11.7 & 10.5 & 19.1 \\
16.4 & 16.1 & 14.0 & 11.6 & 12.9 & 5.5 \\
20.9 & 14.7 & 14.5 & 11.8 & 11.0 & 9.7 \\
21.9 & 17.4 & 17.5 & 8.5 & 14.3 & 10.7 \\
20.6 & 17.8 & 9.4 & 10.1 & 10.7 & 9.3 \\
17.4 & 12.6 & 17.0 & 11.7 & 17.6 & 9.6 \\
\hline
\end{tabular}

Female (age 16-21)

(definitely \& probably)

Year

Youth Poll 1 (Apr 01)

Youth Poll 2 (Aug 01)

Youth Poll 3 (Nov 01)

Youth Poll 4 (Nov 02)

Youth Poll 5 (June 03)

Youth Poll 6 (Nov 03)

Youth Poll 7 (May 04)

Youth Poll 8 (Nov 04)

\begin{tabular}{cccccc}
\multicolumn{7}{c}{ Percent (\%) } \\
\hline $\mathbf{7}$ & Age \\
\hline $\mathbf{1 6}$ & $\mathbf{1 7}$ & $\mathbf{1 8}$ & $\mathbf{1 9}$ & $\mathbf{2 0}$ & $\mathbf{2 1}$ \\
\hline 8.1 & 5.8 & 1.8 & 2.9 & 3.2 & 0.0 \\
8.4 & 8.3 & 7.0 & 2.2 & 4.2 & 2.9 \\
8.0 & 5.8 & 2.3 & 4.4 & 7.4 & 1.1 \\
11.3 & 5.5 & 1.7 & 5.1 & 5.8 & 1.2 \\
7.5 & 8.0 & 6.1 & 4.2 & 4.1 & 2.8 \\
12.6 & 7.7 & 4.8 & 4.0 & 4.0 & 5.2 \\
10.1 & 11.6 & 4.8 & 5.2 & 4.4 & 4.7 \\
7.7 & 4.6 & 5.1 & 3.1 & 1.9 & 5.6 \\
\hline
\end{tabular}

${ }^{13}$ ‡Reporting standard not met (too few cases).

Note: Changes in sample size and stratification were implemented following November 2002 Youth Poll.

Source: Department of Defense Polls, JAMRS, 2001-2004 (Question: FPP10B). 


\section{Army Active Duty Propensity}

JAMRS

TABLE 3-4. Youth Army active duty propensity, by geographic region: $2001-2004^{14}$

\begin{tabular}{|c|c|c|c|c|c|c|c|c|c|}
\hline Male and Female (age & $6-21)$ & & & & rcent ( $\%$ & & & & \\
\hline (definitely \& probably) & & & & Geo & raphic $\mathrm{F}$ & gion & & & \\
\hline Year & $\begin{array}{c}\text { New } \\
\text { England }\end{array}$ & $\begin{array}{c}\text { Mid } \\
\text { Atlantic }\end{array}$ & $\begin{array}{c}\text { East } \\
\text { North } \\
\text { Central }\end{array}$ & $\begin{array}{c}\text { West } \\
\text { North } \\
\text { Central }\end{array}$ & $\begin{array}{c}\text { South } \\
\text { Atlantic }\end{array}$ & $\begin{array}{c}\text { East } \\
\text { South } \\
\text { Central }\end{array}$ & $\begin{array}{c}\text { West } \\
\text { South } \\
\text { Central }\end{array}$ & Mountain & Pacific \\
\hline Youth Poll 1 (Apr 01) & $\ddagger$ & $\ddagger$ & $\ddagger$ & $\ddagger$ & $\ddagger$ & $\ddagger$ & $\ddagger$ & $\ddagger$ & $\ddagger$ \\
\hline Youth Poll 2 (Aug 01) & $\ddagger$ & $\ddagger$ & $\ddagger$ & $\ddagger$ & $\ddagger$ & $\ddagger$ & $\ddagger$ & $\ddagger$ & $\ddagger$ \\
\hline Youth Poll 3 (Nov 01) & $\ddagger$ & $\ddagger$ & $\ddagger$ & $\ddagger$ & $\ddagger$ & $\ddagger$ & $\ddagger$ & $\ddagger$ & $\ddagger$ \\
\hline Youth Poll 4 (Nov 02) & $\ddagger$ & $\ddagger$ & $\ddagger$ & $\ddagger$ & $\ddagger$ & $\ddagger$ & $\ddagger$ & $\ddagger$ & $\ddagger$ \\
\hline Youth Poll 5 (June 03) & 10.1 & 9.2 & 7.2 & 7.9 & 9.8 & 10.2 & 9.7 & 11.6 & 12.2 \\
\hline Youth Poll 6 (Nov 03) & 9.9 & 9.7 & 9.4 & 6.4 & 11.6 & 20.9 & 14.0 & 8.4 & 10.6 \\
\hline Youth Poll 7 (May 04) & 8.8 & 10.1 & 11.9 & 5.4 & 9.9 & 4.4 & 10.3 & 11.5 & 11.8 \\
\hline Youth Poll 8 (Nov 04) & 8.7 & 8.7 & 5.6 & 8.0 & 10.1 & 9.0 & 17.7 & 8.2 & 9.2 \\
\hline Male (age 16-21) & & & & & ercent $(9$ & & & & \\
\hline (definitely \& probably) & & & & Geo & raphic $\mathbf{R}$ & gion & & & \\
\hline Year & $\begin{array}{l}\text { New } \\
\text { England }\end{array}$ & $\begin{array}{c}\text { Mid } \\
\text { Atlantic }\end{array}$ & $\begin{array}{c}\text { East } \\
\text { North } \\
\text { Central } \\
\end{array}$ & $\begin{array}{c}\text { West } \\
\text { North } \\
\text { Central } \\
\end{array}$ & $\begin{array}{c}\text { South } \\
\text { Atlantic }\end{array}$ & $\begin{array}{c}\text { East } \\
\text { South } \\
\text { Central } \\
\end{array}$ & $\begin{array}{c}\text { West } \\
\text { South } \\
\text { Central }\end{array}$ & Mountain & Pacific \\
\hline Youth Poll 1 (Apr 01) & $\ddagger$ & $\neq$ & $\ddagger$ & $\neq$ & $\ddagger$ & $\mp$ & $\ddagger$ & $\neq$ & $\ddagger$ \\
\hline Youth Poll 2 (Aug 01) & $\ddagger$ & $\ddagger$ & $\ddagger$ & $\ddagger$ & $\ddagger$ & $\ddagger$ & $\ddagger$ & $\ddagger$ & $\ddagger$ \\
\hline Youth Poll 3 (Nov 01) & $\ddagger$ & $\ddagger$ & $\ddagger$ & $\ddagger$ & $\ddagger$ & $\ddagger$ & $\ddagger$ & $\ddagger$ & $\ddagger$ \\
\hline Youth Poll 4 (Nov 02) & $\ddagger$ & $\ddagger$ & $\ddagger$ & $\ddagger$ & $\ddagger$ & $\ddagger$ & $\ddagger$ & $\ddagger$ & $\ddagger$ \\
\hline Youth Poll 5 (June 03) & $\ddagger$ & 9.8 & 11.4 & 15.3 & 13.0 & $\ddagger$ & 14.0 & 16.2 & 17.6 \\
\hline Youth Poll 6 (Nov 03) & $\ddagger$ & 12.2 & 13.4 & 10.6 & 16.3 & $\ddagger$ & 18.7 & $\ddagger$ & 13.9 \\
\hline Youth Poll 7 (May 04) & $\ddagger$ & 12.4 & 13.8 & $\ddagger$ & 14.7 & $\ddagger$ & 15.9 & $\ddagger$ & 14.8 \\
\hline Youth Poll 8 (Nov 04) & $\ddagger$ & 13.7 & 7.7 & 12.4 & 17.1 & $\ddagger$ & 25.1 & 13.7 & 12.8 \\
\hline Female (age 16-21) & & & & & ercent $(9$ & & & & \\
\hline (definitely \& probably) & & & & Geo & raphic $R$ & gion & & & \\
\hline Year & $\begin{array}{c}\text { New } \\
\text { England }\end{array}$ & $\begin{array}{c}\text { Mid } \\
\text { Atlantic } \\
\end{array}$ & $\begin{array}{c}\text { East } \\
\text { North } \\
\text { Central } \\
\end{array}$ & $\begin{array}{c}\text { West } \\
\text { North } \\
\text { Central } \\
\end{array}$ & $\begin{array}{c}\text { South } \\
\text { Atlantic } \\
\end{array}$ & $\begin{array}{c}\text { East } \\
\text { South } \\
\text { Central }\end{array}$ & $\begin{array}{c}\text { West } \\
\text { South } \\
\text { Central }\end{array}$ & Mountain & Pacific \\
\hline Youth Poll 1 (Apr 01) & $\ddagger$ & 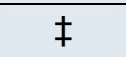 & $\ddagger$ & $\ddagger$ & $\ddagger$ & $\neq$ & $\ddagger$ & $\mp$ & $\ddagger$ \\
\hline Youth Poll 2 (Aug 01) & $\ddagger$ & $\ddagger$ & $\ddagger$ & $\ddagger$ & $\ddagger$ & $\ddagger$ & $\ddagger$ & $\ddagger$ & $\ddagger$ \\
\hline Youth Poll 3 (Nov 01) & $\ddagger$ & $\ddagger$ & $\ddagger$ & $\ddagger$ & $\ddagger$ & $\ddagger$ & $\ddagger$ & $\ddagger$ & $\ddagger$ \\
\hline Youth Poll 4 (Nov 02) & $\ddagger$ & $\ddagger$ & $\ddagger$ & $\ddagger$ & $\ddagger$ & $\ddagger$ & $\ddagger$ & $\ddagger$ & $\ddagger$ \\
\hline Youth Poll 5 (June 03) & $\ddagger$ & 8.5 & 4.1 & 1.0 & 6.4 & $\ddagger$ & 5.8 & 6.0 & 5.9 \\
\hline Youth Poll 6 (Nov 03) & $\ddagger$ & 7.4 & 4.9 & 2.0 & 6.9 & 9.2 & 9.4 & 6.5 & 7.2 \\
\hline Youth Poll 7 (May 04) & $\ddagger$ & 7.3 & 10.0 & $\ddagger$ & 5.4 & $\ddagger$ & 5.4 & $\ddagger$ & 8.9 \\
\hline Youth Poll 8 (Nov 04) & $\ddagger$ & 3.7 & 3.7 & 2.1 & 3.6 & $\ddagger$ & 11.4 & 2.7 & 4.8 \\
\hline
\end{tabular}

${ }^{14}$ ‡Reporting standard not met (too few cases).

Note: Changes in sample size and stratification were implemented following November 2002 Youth Poll.

Source: Department of Defense Polls, JAMRS, 2001-2004 (Question: FPP10B). 


\section{Army Active Duty Propensity}

JAMRS

TABLE 3-5. Youth Army active duty propensity, by high school grades: $2001-2004^{15}$

\begin{tabular}{|c|c|c|c|c|c|c|c|}
\hline \multicolumn{4}{|c|}{ Male and Female (age $16-21)$} & \multicolumn{4}{|c|}{ Percent (\%) } \\
\hline \multirow{2}{*}{$\begin{array}{l}\text { (definitely \& probably) } \\
\text { Year }\end{array}$} & \multicolumn{7}{|c|}{ High School Grades } \\
\hline & $\begin{array}{l}\text { Mostly } \\
\text { A's }\end{array}$ & $\begin{array}{l}\text { Mostly A's } \\
\text { \& B's }\end{array}$ & $\begin{array}{l}\text { Mostly } \\
\text { B's }\end{array}$ & $\begin{array}{l}\text { Mostly B's } \\
\text { \& C's }\end{array}$ & $\begin{array}{l}\text { Mostly } \\
\text { C's }\end{array}$ & $\begin{array}{l}\text { Mostly C's } \\
\text { \& D's }\end{array}$ & $\begin{array}{l}\text { Mostly D's } \\
\text { and Lower }\end{array}$ \\
\hline Youth Poll 1 (Apr 01) & 1.5 & 4.3 & 6.1 & 9.2 & $\ddagger$ & 10.3 & $\ddagger$ \\
\hline Youth Poll 2 (Aug 01) & 4.6 & 5.3 & 4.5 & 8.9 & $\ddagger$ & 17.9 & $\ddagger$ \\
\hline Youth Poll 3 (Nov 01) & 3.3 & 6.8 & 10.7 & 15.1 & $\ddagger$ & $\ddagger$ & $\ddagger$ \\
\hline Youth Poll 4 (Nov 02) & 4.6 & 4.2 & 6.7 & 15.0 & 18.8 & $\ddagger$ & $\ddagger$ \\
\hline Youth Poll 5 (June 03) & 3.8 & 8.4 & 7.4 & 14.0 & 11.0 & 21.8 & $\ddagger$ \\
\hline Youth Poll 6 (Nov 03) & 5.3 & 9.9 & 9.0 & 15.0 & 7.6 & 24.0 & $\ddagger$ \\
\hline Youth Poll 7 (May 04) & 2.0 & 8.5 & 8.7 & 13.8 & 17.0 & 18.7 & $\ddagger$ \\
\hline Youth Poll 8 (Nov 04) & 3.7 & 8.6 & 5.4 & 14.0 & 12.4 & 18.3 & $\ddagger$ \\
\hline Male (age 16-21) & \multicolumn{7}{|c|}{ Percent (\%) } \\
\hline (definitely \& probably) & \multicolumn{7}{|c|}{ High School Grades } \\
\hline Year & $\begin{array}{c}\text { Mostly } \\
\text { A's }\end{array}$ & $\begin{array}{l}\text { Mostly A's } \\
\text { \& B's }\end{array}$ & $\begin{array}{l}\text { Mostly } \\
\text { B's }\end{array}$ & $\begin{array}{l}\text { Mostly B's } \\
\text { \& C's }\end{array}$ & $\begin{array}{l}\text { Mostly } \\
\text { C's }\end{array}$ & $\begin{array}{l}\text { Mostly C's } \\
\text { \& D's }\end{array}$ & $\begin{array}{l}\text { Mostly D's } \\
\text { and Lower }\end{array}$ \\
\hline Youth Poll 1 (Apr 01) & $\ddagger$ & 6.8 & $\ddagger$ & 10.3 & $\ddagger$ & $\ddagger$ & $\ddagger$ \\
\hline Youth Poll 2 (Aug 01) & $\ddagger$ & 6.2 & 5.1 & 11.0 & $\ddagger$ & $\ddagger$ & $\ddagger$ \\
\hline Youth Poll 3 (Nov 01) & 3.3 & 11.1 & 16.1 & 21.8 & $\ddagger$ & $\ddagger$ & $\ddagger$ \\
\hline Youth Poll 4 (Nov 02) & $\ddagger$ & 4.9 & 8.8 & 18.4 & $\ddagger$ & $\ddagger$ & $\ddagger$ \\
\hline Youth Poll 5 (June 03) & 7.5 & 11.0 & 11.9 & 18.3 & 11.1 & 25.2 & $\ddagger$ \\
\hline Youth Poll 6 (Nov 03) & 7.6 & 12.8 & 12.3 & 22.4 & 7.9 & 29.2 & $\ddagger$ \\
\hline Youth Poll 7 (May 04) & 1.8 & 11.5 & 11.1 & 15.8 & $\ddagger$ & 22.0 & $\ddagger$ \\
\hline Youth Poll 8 (Nov 04) & 6.1 & 14.2 & 7.7 & 19.2 & 14.2 & $\ddagger$ & $\ddagger$ \\
\hline Females (age 16-21) & \multicolumn{7}{|c|}{ Percent (\%) } \\
\hline (definitely \& probably) & \multicolumn{7}{|c|}{ High School Grades } \\
\hline Year & $\begin{array}{c}\text { Mostly } \\
\text { A's }\end{array}$ & $\begin{array}{l}\text { Mostly A's } \\
\text { \& B's }\end{array}$ & $\begin{array}{l}\text { Mostly } \\
\text { B's }\end{array}$ & $\begin{array}{l}\text { Mostly B's } \\
\text { \& C's }\end{array}$ & $\begin{array}{l}\text { Mostly } \\
\text { C's }\end{array}$ & $\begin{array}{l}\text { Mostly C's } \\
\text { \& D's }\end{array}$ & $\begin{array}{l}\text { Mostly D's } \\
\text { and Lower }\end{array}$ \\
\hline Youth Poll 1 (Apr 01) & .9 & 2.6 & $\ddagger$ & 8.0 & $\ddagger$ & $\ddagger$ & $\ddagger$ \\
\hline Youth Poll 2 (Aug 01) & 3.4 & 4.6 & 4.0 & 6.7 & $\ddagger$ & $\ddagger$ & $\ddagger$ \\
\hline Youth Poll 3 (Nov 01) & 3.3 & 3.8 & 5.2 & 7.4 & $\ddagger$ & $\ddagger$ & $\ddagger$ \\
\hline Youth Poll 4 (Nov 02) & 2.6 & 3.7 & 4.9 & 10.0 & $\ddagger$ & $\ddagger$ & $\ddagger$ \\
\hline Youth Poll 5 (June 03) & 1.3 & 6.5 & 3.3 & 7.3 & $\ddagger$ & $\ddagger$ & $\ddagger$ \\
\hline Youth Poll 6 (Nov 03) & 3.7 & 7.8 & 5.7 & 6.3 & $\ddagger$ & $\ddagger$ & $\ddagger$ \\
\hline Youth Poll 7 (May 04) & 2.2 & 6.4 & 5.9 & 11.2 & $\ddagger$ & $\ddagger$ & $\ddagger$ \\
\hline Youth Poll 8 (Nov 04) & 2.2 & 3.9 & 3.1 & 7.0 & $\ddagger$ & $\ddagger$ & $\ddagger$ \\
\hline
\end{tabular}

${ }^{15} \ddagger$ Reporting standard not met (too few cases).

Note: Changes in sample size and stratification were implemented following November 2002 Youth Poll.

Source: Department of Defense Polls, JAMRS, 2001-2004 (Question: FPP10B). 


\section{Navy Active Duty Propensity}

TABLE 4-1. Youth Navy active duty propensity: $2001-2004^{16}$

Male and Female (age 16-21)

Percent (\%)

\begin{tabular}{lcccc}
\cline { 2 - 4 } Year & Definitely & Probably & Probably Not & Definitely Not \\
\hline Youth Poll 1 (Apr 01) & 1.0 & 5.6 & 23.0 & 70.3 \\
Youth Poll 2 (Aug 01) & 1.2 & 5.8 & 24.9 & 68.0 \\
Youth Poll 3 (Nov 01) & 1.3 & 7.3 & 29.9 & 61.3 \\
Youth Poll 4 (Nov 02) & 1.0 & 5.9 & 27.5 & 65.6 \\
Youth Poll 5 (June 03) & 1.4 & 7.1 & 27.7 & 63.7 \\
Youth Poll 6 (Nov 03) & 1.4 & 7.3 & 27.0 & 64.3 \\
Youth Poll 7 (May 04) & 1.3 & 7.0 & 29.7 & 61.9 \\
Youth Poll 8 (Nov 04) & 1.3 & 6.4 & 28.0 & 64.3 \\
\hline
\end{tabular}

Male (age 16-21)

Percent (\%)

\begin{tabular}{lcccc}
\cline { 2 - 4 } Year & Definitely & Probably & Probably Not & Definitely Not \\
\hline Youth Poll 1 (Apr 01) & 1.7 & 7.2 & 30.3 & 60.7 \\
Youth Poll 2 (Aug 01) & 1.6 & 7.4 & 31.2 & 59.8 \\
Youth Poll 3 (Nov 01) & 2.2 & 10.1 & 36.7 & 51.0 \\
Youth Poll 4 (Nov 02) & .9 & 7.7 & 32.2 & 59.2 \\
Youth Poll 5 (June 03) & 1.9 & 9.4 & 33.6 & 55.0 \\
Youth Poll 6 (Nov 03) & 1.8 & 9.4 & 34.0 & 54.7 \\
Youth Poll 7 (May 04) & 1.6 & 9.1 & 36.0 & 53.2 \\
Youth Poll 8 (Nov 04) & 2.0 & 8.6 & 34.6 & 54.8 \\
\hline
\end{tabular}

Female (age 16-21)

Percent (\%)

\begin{tabular}{lcccc}
\cline { 2 - 4 } Year & Definitely & Probably & Probably Not & Definitely Not \\
\hline Youth Poll 1 (Apr 01) & .3 & 4.1 & 16.2 & 79.3 \\
Youth Poll 2 (Aug 01) & .9 & 4.2 & 18.9 & 75.8 \\
Youth Poll 3 (Nov 01) & .5 & 4.7 & 23.6 & 71.0 \\
Youth Poll 4 (Nov 02) & 1.1 & 4.1 & 23.0 & 71.7 \\
Youth Poll 5 (June 03) & .9 & 4.8 & 21.6 & 72.6 \\
Youth Poll 6 (Nov 03) & 1.0 & 5.2 & 19.8 & 74.0 \\
Youth Poll 7 (May 04) & .9 & 4.8 & 23.3 & 70.9 \\
Youth Poll 8 (Nov 04) & .5 & 4.1 & 21.3 & 74.0 \\
\hline
\end{tabular}

${ }^{16}$ ‡Reporting standard not met (too few cases).

Note: Changes in sample size and stratification were implemented following November 2002 Youth Poll.

Source: Department of Defense Polls, JAMRS, 2001-2004 (Question: FPP10E). 


\section{Navy Active Duty Propensity}

TABLE 4-2. Youth Navy active duty propensity, by race/ethnicity: $2001-2004^{17}$

\begin{tabular}{|c|c|c|c|c|}
\hline \multirow{3}{*}{$\begin{array}{l}\text { Male and Female (age 16-21) } \\
\text { (definitely \& probably) } \\
\text { Year }\end{array}$} & \multicolumn{4}{|c|}{ Percent (\%) } \\
\hline & \multicolumn{4}{|c|}{ Race/ethnicity } \\
\hline & Total & White & Black & Hispanic \\
\hline Youth Poll 1 (Apr 01) & 6.6 & 4.3 & 13.2 & 10.0 \\
\hline Youth Poll 2 (Aug 01) & 7.0 & 3.6 & 13.8 & 12.3 \\
\hline Youth Poll 3 (Nov 01) & 8.6 & 6.1 & 9.5 & 19.5 \\
\hline Youth Poll 4 (Nov 02) & 6.9 & 5.0 & 8.9 & 10.2 \\
\hline Youth Poll 5 (June 03) & 8.5 & 6.0 & 12.5 & 14.6 \\
\hline Youth Poll 6 (Nov 03) & 8.7 & 5.5 & 13.7 & 16.1 \\
\hline Youth Poll 7 (May 04) & 8.2 & 5.2 & 12.0 & 15.9 \\
\hline Youth Poll 8 (Nov 04) & 7.6 & 5.4 & 9.9 & 12.1 \\
\hline Male (age 16-21) & \multicolumn{4}{|c|}{ Percent (\%) } \\
\hline (definitely \& probably) & \multicolumn{4}{|c|}{ Race/ethnicity } \\
\hline Year & Total & White & Black & Hispanic \\
\hline Youth Poll 1 (Apr 01) & 8.9 & 6.3 & 19.5 & 11.4 \\
\hline Youth Poll 2 (Aug 01) & 9.0 & 5.0 & 19.8 & 13.9 \\
\hline Youth Poll 3 (Nov 01) & 12.3 & 8.8 & 12.7 & 28.2 \\
\hline Youth Poll 4 (Nov 02) & 8.6 & 7.0 & 9.1 & 11.6 \\
\hline Youth Poll 5 (June 03) & 11.3 & 8.3 & 16.4 & 18.7 \\
\hline Youth Poll 6 (Nov 03) & 11.3 & 7.6 & 16.8 & 20.6 \\
\hline Youth Poll 7 (May 04) & 10.7 & 7.6 & 12.4 & 19.4 \\
\hline Youth Poll 8 (Nov 04) & 10.6 & 8.2 & 14.8 & 14.7 \\
\hline Female (age 16-21) & \multicolumn{4}{|c|}{ Percent (\%) } \\
\hline (definitely \& probably) & \multicolumn{4}{|c|}{ Race/ethnicity } \\
\hline Year & Total & White & Black & Hispanic \\
\hline Youth Poll 1 (Apr 01) & 4.4 & 2.2 & 9.0 & 8.9 \\
\hline Youth Poll 2 (Aug 01) & 5.1 & 2.2 & 10.0 & 10.6 \\
\hline Youth Poll 3 (Nov 01) & 5.2 & 3.3 & 7.6 & 10.3 \\
\hline Youth Poll 4 (Nov 02) & 5.2 & 3.0 & 8.6 & 9.2 \\
\hline Youth Poll 5 (June 03) & 5.7 & 3.7 & 9.3 & 10.4 \\
\hline Youth Poll 6 (Nov 03) & 6.2 & 3.2 & 11.1 & 11.9 \\
\hline Youth Poll 7 (May 04) & 5.7 & 2.6 & 11.6 & 12.3 \\
\hline Youth Poll 8 (Nov 04) & 4.6 & 2.3 & 6.6 & 9.5 \\
\hline
\end{tabular}

\footnotetext{
${ }^{17}$ Due to relatively small sample sizes, American Indian, Alaska Native, Asian, and Pacific Islander are included in the total but are not shown separately. Black includes African American, and Hispanic includes Latino. Race categories exclude Hispanic origin unless specified otherwise.

‡Reporting standard not met (too few cases).

Note: Changes in sample size and stratification were implemented following November 2002 Youth Poll. Source: Department of Defense Polls, JAMRS, 2001-2004 (Question: FPP10E).
} 


\section{Navy Active Duty Propensity}

TABLE 4-3. Youth Navy active duty propensity, by age: $2001-2004^{18}$

\begin{tabular}{lccccccc} 
Male and Female (age 16-21) & \multicolumn{7}{c}{ Percent (\%) } \\
\cline { 2 - 7 } $\begin{array}{l}\text { (definitely \& probably) } \\
\text { Year }\end{array}$ & $\mathbf{1 6}$ & $\mathbf{1 7}$ & $\mathbf{1 8}$ & $\mathbf{1 9}$ & $\mathbf{2 0}$ & $\mathbf{2 1}$ \\
\hline & 8.6 & 7.4 & 3.9 & 7.7 & 7.2 & 4.5 \\
\hline Youth Poll 1 (Apr 01) & 13.3 & 8.0 & 7.5 & 5.5 & 5.3 & 1.9 \\
Youth Poll 2 (Aug 01) & 11.2 & 12.3 & 8.5 & 5.9 & 9.8 & 3.9 \\
Youth Poll 3 (Nov 01) & 10.7 & 7.5 & 4.8 & 6.5 & 7.8 & 3.8 \\
Youth Poll 4 (Nov 02) & 13.3 & 9.5 & 8.5 & 7.0 & 6.3 & 6.0 \\
Youth Poll 5 (June 03) & 14.0 & 10.9 & 7.8 & 7.2 & 5.9 & 5.6 \\
Youth Poll 6 (Nov 03) & 11.3 & 10.5 & 7.6 & 7.8 & 5.7 & 6.0 \\
Youth Poll 7 (May 04) & 12.1 & 9.5 & 6.9 & 6.0 & 4.4 & 6.0 \\
Youth Poll 8 (Nov 04) & & & & & & \\
\hline
\end{tabular}

\section{Male (age 16-21)}

(definitely \& probably)

\section{Year}

Youth Poll 1 (Apr 01)

Youth Poll 2 (Aug 01)

Youth Poll 3 (Nov 01)

Youth Poll 4 (Nov 02)

Youth Poll 5 (June 03)

Youth Poll 6 (Nov 03)

Youth Poll 7 (May 04)

Youth Poll 8 (Nov 04)

\begin{tabular}{cccccc}
\multicolumn{7}{c}{ Percent (\%) } \\
\hline $\mathbf{7}$ & Age & $\mathbf{2 1}$ \\
\hline 16 & $\mathbf{1 7}$ & $\mathbf{1 8}$ & $\mathbf{1 9}$ & $\mathbf{2 0}$ & $\mathbf{2 1}$ \\
10.5 & 7.8 & 6.6 & 12.5 & 8.1 & 7.4 \\
13.8 & 10.4 & 8.8 & 8.3 & 8.4 & 2.1 \\
15.4 & 15.5 & 13.9 & 8.8 & 14.8 & 4.7 \\
11.0 & 10.4 & 6.9 & 8.4 & 8.9 & 5.5 \\
16.3 & 11.5 & 12.4 & 10.3 & 9.2 & 7.4 \\
16.6 & 12.3 & 11.1 & 11.9 & 8.5 & 6.4 \\
14.4 & 13.5 & 9.4 & 7.9 & 9.5 & 8.9 \\
15.3 & 12.8 & 10.7 & 9.4 & 5.5 & 8.7 \\
\hline
\end{tabular}

\section{Female (age 16-21)}

(definitely \& probably)

\section{Year}

Youth Poll 1 (Apr 01)

Youth Poll 2 (Aug 01)

Youth Poll 3 (Nov 01)

Youth Poll 4 (Nov 02)

Youth Poll 5 (June 03)

Youth Poll 6 (Nov 03)

Youth Poll 7 (May 04)

Youth Poll 8 (Nov 04)

\begin{tabular}{cccccc}
\multicolumn{7}{c}{ Percent (\%) } \\
\hline $\mathbf{7}$ & Age \\
\hline 16 & $\mathbf{1 7}$ & $\mathbf{1 8}$ & $\mathbf{1 9}$ & $\mathbf{2 0}$ & $\mathbf{2 1}$ \\
\hline 6.8 & 6.9 & 1.4 & 2.7 & 6.4 & 2.5 \\
12.6 & 5.4 & 6.4 & 3.0 & 2.8 & 1.8 \\
7.2 & 8.7 & 2.9 & 3.5 & 5.7 & 3.2 \\
10.3 & 4.8 & 2.6 & 4.8 & 6.6 & 2.5 \\
10.1 & 7.3 & 4.5 & 3.8 & 3.2 & 4.7 \\
11.5 & 9.3 & 4.7 & 2.4 & 3.5 & 4.8 \\
8.1 & 7.6 & 5.6 & 7.7 & 1.7 & 3.2 \\
8.6 & 6.1 & 3.0 & 2.7 & 3.2 & 3.3 \\
\hline
\end{tabular}

${ }^{18}$ ‡Reporting standard not met (too few cases).

Note: Changes in sample size and stratification were implemented following November 2002 Youth Poll.

Source: Department of Defense Polls, JAMRS, 2001-2004 (Question: FPP10E). 


\section{Navy Active Duty Propensity}

JAMRS

TABLE 4-4. Youth Navy active duty propensity, by geographic region: $2001-2004^{19}$

\begin{tabular}{|c|c|c|c|c|c|c|c|c|c|}
\hline \multirow{3}{*}{$\begin{array}{l}\text { Male and Female (age } \\
\text { (definitely \& probably) } \\
\text { Year }\end{array}$} & \multicolumn{9}{|c|}{ Percent (\%) } \\
\hline & \multicolumn{9}{|c|}{ Geographic Region } \\
\hline & $\begin{array}{c}\text { New } \\
\text { England }\end{array}$ & $\begin{array}{c}\text { Mid } \\
\text { Atlantic }\end{array}$ & $\begin{array}{c}\text { East } \\
\text { North } \\
\text { Central }\end{array}$ & $\begin{array}{c}\text { West } \\
\text { North } \\
\text { Central }\end{array}$ & $\begin{array}{c}\text { South } \\
\text { Atlantic }\end{array}$ & $\begin{array}{c}\text { East } \\
\text { South } \\
\text { Central }\end{array}$ & $\begin{array}{c}\text { West } \\
\text { South } \\
\text { Central }\end{array}$ & Mountain & Pacific \\
\hline Youth Poll 1 (Apr 01) & $\ddagger$ & $\ddagger$ & $\ddagger$ & $\ddagger$ & $\ddagger$ & $\ddagger$ & $\ddagger$ & $\ddagger$ & $\ddagger$ \\
\hline Youth Poll 2 (Aug 01) & $\ddagger$ & $\ddagger$ & $\ddagger$ & $\ddagger$ & $\ddagger$ & $\ddagger$ & $\ddagger$ & $\ddagger$ & $\ddagger$ \\
\hline Youth Poll 3 (Nov 01) & $\ddagger$ & $\ddagger$ & $\ddagger$ & $\ddagger$ & $\ddagger$ & $\ddagger$ & $\ddagger$ & $\ddagger$ & $\ddagger$ \\
\hline Youth Poll 4 (Nov 02) & $\ddagger$ & $\ddagger$ & $\ddagger$ & $\ddagger$ & $\ddagger$ & $\ddagger$ & $\ddagger$ & $\ddagger$ & $\ddagger$ \\
\hline Youth Poll 5 (June 03) & 11.1 & 6.7 & 6.8 & 7.5 & 8.8 & 8.0 & 9.8 & 8.3 & 10.3 \\
\hline Youth Poll 6 (Nov 03) & 3.7 & 6.8 & 6.2 & 7.5 & 10.0 & 8.1 & 12.7 & 7.0 & 11.2 \\
\hline Youth Poll 7 (May 04) & 9.1 & 7.1 & 6.1 & 3.7 & 7.0 & 7.0 & 8.4 & 11.0 & 12.6 \\
\hline Youth Poll 8 (Nov 04) & 6.8 & 8.2 & 4.0 & 5.0 & 8.1 & 7.6 & 7.3 & 9.2 & 11.3 \\
\hline Male (age 16-21) & \multicolumn{9}{|c|}{ Percent (\%) } \\
\hline (definitely \& probably) & \multicolumn{9}{|c|}{ Geographic Region } \\
\hline Year & $\begin{array}{c}\text { New } \\
\text { England }\end{array}$ & $\begin{array}{c}\text { Mid } \\
\text { Atlantic } \\
\end{array}$ & $\begin{array}{c}\text { East } \\
\text { North } \\
\text { Central }\end{array}$ & $\begin{array}{c}\text { West } \\
\text { North } \\
\text { Central }\end{array}$ & $\begin{array}{c}\text { South } \\
\text { Atlantic }\end{array}$ & $\begin{array}{c}\text { East } \\
\text { South } \\
\text { Central }\end{array}$ & $\begin{array}{c}\text { West } \\
\text { South } \\
\text { Central }\end{array}$ & Mountain & Pacific \\
\hline Youth Poll 1 (Apr 01) & $\ddagger$ & $\ddagger$ & $\ddagger$ & $\ddagger$ & $\ddagger$ & $\ddagger$ & $\ddagger$ & $\ddagger$ & $\ddagger$ \\
\hline Youth Poll 2 (Aug 01) & $\ddagger$ & $\ddagger$ & $\ddagger$ & $\ddagger$ & $\ddagger$ & $\ddagger$ & $\ddagger$ & $\ddagger$ & $\ddagger$ \\
\hline Youth Poll 3 (Nov 01) & $\ddagger$ & $\ddagger$ & $\ddagger$ & $\ddagger$ & $\ddagger$ & $\ddagger$ & $\ddagger$ & $\ddagger$ & $\ddagger$ \\
\hline Youth Poll 4 (Nov 02) & $\ddagger$ & $\ddagger$ & $\ddagger$ & $\ddagger$ & $\ddagger$ & $\ddagger$ & $\ddagger$ & $\ddagger$ & $\ddagger$ \\
\hline Youth Poll 5 (June 03) & $\ddagger$ & 9.0 & 9.4 & 12.1 & 13.3 & $\ddagger$ & 13.9 & 10.5 & 12.2 \\
\hline Youth Poll 6 (Nov 03) & $\ddagger$ & 7.5 & 7.7 & 12.5 & 11.1 & $\ddagger$ & 17.8 & $\ddagger$ & 14.8 \\
\hline Youth Poll 7 (May 04) & $\ddagger$ & 10.2 & 8.0 & $\ddagger$ & 8.1 & $\ddagger$ & 11.5 & $\ddagger$ & 18.1 \\
\hline Youth Poll 8 (Nov 04) & $\ddagger$ & 13.6 & 4.5 & 7.3 & 11.6 & $\ddagger$ & 9.4 & 13.2 & 14.0 \\
\hline Female (age 16-21) & \multicolumn{9}{|c|}{ Percent (\%) } \\
\hline (definitely \& probably) & \multicolumn{9}{|c|}{ Geographic Region } \\
\hline Year & $\begin{array}{c}\text { New } \\
\text { England }\end{array}$ & $\begin{array}{c}\text { Mid } \\
\text { Atlantic } \\
\end{array}$ & $\begin{array}{c}\text { East } \\
\text { North } \\
\text { Central }\end{array}$ & $\begin{array}{c}\text { West } \\
\text { North } \\
\text { Central } \\
\end{array}$ & $\begin{array}{c}\text { South } \\
\text { Atlantic }\end{array}$ & $\begin{array}{c}\text { East } \\
\text { South } \\
\text { Central }\end{array}$ & $\begin{array}{c}\text { West } \\
\text { South } \\
\text { Central }\end{array}$ & Mountain & Pacific \\
\hline Youth Poll 1 (Apr 01) & $\ddagger$ & $\ddagger$ & $\ddagger$ & $\ddagger$ & $\ddagger$ & $\ddagger$ & $\ddagger$ & $\ddagger$ & $\ddagger$ \\
\hline Youth Poll 2 (Aug 01) & $\ddagger$ & $\ddagger$ & $\ddagger$ & $\ddagger$ & $\ddagger$ & $\ddagger$ & $\ddagger$ & $\ddagger$ & $\ddagger$ \\
\hline Youth Poll 3 (Nov 01) & $\ddagger$ & $\ddagger$ & $\ddagger$ & $\ddagger$ & $\ddagger$ & $\ddagger$ & $\ddagger$ & $\ddagger$ & $\ddagger$ \\
\hline Youth Poll 4 (Nov 02) & $\ddagger$ & $\ddagger$ & $\ddagger$ & $\ddagger$ & $\ddagger$ & $\ddagger$ & $\ddagger$ & $\ddagger$ & $\ddagger$ \\
\hline Youth Poll 5 (June 03) & $\ddagger$ & 3.8 & 4.9 & 3.3 & 4.1 & $\ddagger$ & 6.2 & 5.6 & 8.1 \\
\hline Youth Poll 6 (Nov 03) & $\ddagger$ & 6.1 & 4.5 & 2.4 & 8.8 & 6.0 & 7.8 & 4.2 & 7.4 \\
\hline Youth Poll 7 (May 04) & $\ddagger$ & 3.4 & 4.3 & $\ddagger$ & 6.0 & $\ddagger$ & 5.7 & $\ddagger$ & 7.3 \\
\hline Youth Poll 8 (Nov 04) & $\ddagger$ & 2.7 & 3.5 & 1.9 & 4.8 & $\ddagger$ & 5.5 & 5.2 & 8.0 \\
\hline
\end{tabular}

${ }^{19}$ ‡Reporting standard not met (too few cases).

Note: Changes in sample size and stratification were implemented following November 2002 Youth Poll.

Source: Department of Defense Polls, JAMRS, 2001-2004 (Question: FPP10E). 


\section{Navy Active Duty Propensity}

JAMRS

TABLE 4-5. Youth Navy active duty propensity, by high school grades: $2001-2004^{20}$

\begin{tabular}{|c|c|c|c|c|c|c|c|}
\hline \multicolumn{4}{|c|}{ Male and Female (age $16-21)$} & \multicolumn{4}{|c|}{ Percent (\%) } \\
\hline \multirow{2}{*}{$\begin{array}{l}\text { (definitely \& probably) } \\
\text { Year }\end{array}$} & \multicolumn{7}{|c|}{ High School Grades } \\
\hline & $\begin{array}{c}\text { Mostly } \\
\text { A's }\end{array}$ & $\begin{array}{l}\text { Mostly A's } \\
\text { \& B's }\end{array}$ & $\begin{array}{l}\text { Mostly } \\
\text { B's }\end{array}$ & $\begin{array}{c}\text { Mostly B's } \\
\text { \& C's }\end{array}$ & $\begin{array}{l}\text { Mostly } \\
\text { C's }\end{array}$ & $\begin{array}{l}\text { Mostly C's } \\
\text { \& D's }\end{array}$ & $\begin{array}{l}\text { Mostly D's } \\
\text { and Lower }\end{array}$ \\
\hline Youth Poll 1 (Apr 01) & 2.6 & 4.9 & 5.9 & 9.2 & $\ddagger$ & 11.6 & $\ddagger$ \\
\hline Youth Poll 2 (Aug 01) & 3.7 & 6.3 & 4.3 & 9.2 & $\ddagger$ & 10.4 & $\ddagger$ \\
\hline Youth Poll 3 (Nov 01) & 5.7 & 6.7 & 9.4 & 11.6 & $\ddagger$ & $\ddagger$ & $\ddagger$ \\
\hline Youth Poll 4 (Nov 02) & 3.9 & 4.0 & 5.7 & 10.1 & 12.8 & $\ddagger$ & $\ddagger$ \\
\hline Youth Poll 5 (June 03) & 3.5 & 8.7 & 6.9 & 10.9 & 11.0 & 13.9 & $\ddagger$ \\
\hline Youth Poll 6 (Nov 03) & 4.8 & 9.0 & 7.9 & 10.0 & 8.9 & 13.5 & $\ddagger$ \\
\hline Youth Poll 7 (May 04) & 3.4 & 7.4 & 9.9 & 9.6 & 8.3 & 14.8 & $\ddagger$ \\
\hline Youth Poll 8 (Nov 04) & 4.8 & 6.3 & 6.1 & 11.3 & 7.2 & 13.3 & $\ddagger$ \\
\hline Male (age 16-21) & \multicolumn{7}{|c|}{ Percent (\%) } \\
\hline (definitely \& probably) & \multicolumn{7}{|c|}{ High School Grades } \\
\hline Year & $\begin{array}{c}\text { Mostly } \\
\text { A's }\end{array}$ & $\begin{array}{l}\text { Mostly A's } \\
\text { \& B's }\end{array}$ & $\begin{array}{l}\text { Mostly } \\
\text { B's }\end{array}$ & $\begin{array}{l}\text { Mostly B's } \\
\text { \& C's }\end{array}$ & $\begin{array}{l}\text { Mostly } \\
\text { C's }\end{array}$ & $\begin{array}{l}\text { Mostly C's } \\
\text { \& D's }\end{array}$ & $\begin{array}{l}\text { Mostly D's } \\
\text { and Lower }\end{array}$ \\
\hline Youth Poll 1 (Apr 01) & $\ddagger$ & 8.1 & $\ddagger$ & 11.6 & $\ddagger$ & $\ddagger$ & $\ddagger$ \\
\hline Youth Poll 2 (Aug 01) & $\ddagger$ & 6.9 & 6.0 & 12.0 & $\ddagger$ & $\ddagger$ & $\ddagger$ \\
\hline Youth Poll 3 (Nov 01) & 3.8 & 9.5 & 15.5 & 16.2 & $\ddagger$ & $\ddagger$ & $\ddagger$ \\
\hline Youth Poll 4 (Nov 02) & $\ddagger$ & 5.6 & 5.6 & 9.2 & $\ddagger$ & $\ddagger$ & $\ddagger$ \\
\hline Youth Poll 5 (June 03) & 3.8 & 11.7 & 11.3 & 13.9 & 10.5 & 15.4 & $\ddagger$ \\
\hline Youth Poll 6 (Nov 03) & 7.1 & 12.0 & 10.4 & 11.9 & 11.2 & 14.5 & $\ddagger$ \\
\hline Youth Poll 7 (May 04) & 4.2 & 9.7 & 13.6 & 11.2 & $\ddagger$ & 18.2 & $\ddagger$ \\
\hline Youth Poll 8 (Nov 04) & 8.0 & 7.6 & 9.3 & 14.8 & 9.5 & $\ddagger$ & $\ddagger$ \\
\hline Females (age 16-21) & \multicolumn{7}{|c|}{ Percent (\%) } \\
\hline (definitely \& probably) & \multicolumn{7}{|c|}{ High School Grades } \\
\hline Year & $\begin{array}{c}\text { Mostly } \\
\text { A's }\end{array}$ & $\begin{array}{l}\text { Mostly A's } \\
\text { \& B's }\end{array}$ & $\begin{array}{l}\text { Mostly } \\
\text { B's }\end{array}$ & $\begin{array}{l}\text { Mostly B's } \\
\text { \& C's }\end{array}$ & $\begin{array}{l}\text { Mostly } \\
\text { C's }\end{array}$ & $\begin{array}{l}\text { Mostly C's } \\
\text { \& D's }\end{array}$ & $\begin{array}{l}\text { Mostly D's } \\
\text { and Lower }\end{array}$ \\
\hline Youth Poll 1 (Apr 01) & 3.7 & 2.7 & $\ddagger$ & 6.3 & $\ddagger$ & $\ddagger$ & $\ddagger$ \\
\hline Youth Poll 2 (Aug 01) & 2.4 & 5.7 & 2.9 & 6.2 & $\ddagger$ & $\ddagger$ & $\ddagger$ \\
\hline Youth Poll 3 (Nov 01) & 7.0 & 4.8 & 3.1 & 6.3 & $\ddagger$ & $\ddagger$ & $\ddagger$ \\
\hline Youth Poll 4 (Nov 02) & 1.3 & 2.9 & 5.8 & 11.4 & $\ddagger$ & $\ddagger$ & $\ddagger$ \\
\hline Youth Poll 5 (June 03) & 3.4 & 6.5 & 2.8 & 6.3 & $\ddagger$ & $\ddagger$ & $\ddagger$ \\
\hline Youth Poll 6 (Nov 03) & 3.1 & 6.7 & 5.4 & 7.9 & $\ddagger$ & $\ddagger$ & $\ddagger$ \\
\hline Youth Poll 7 (May 04) & 2.8 & 5.9 & 5.5 & 7.6 & $\ddagger$ & $\ddagger$ & $\ddagger$ \\
\hline Youth Poll 8 (Nov 04) & 2.8 & 5.1 & 2.8 & 6.5 & $\ddagger$ & $\ddagger$ & $\ddagger$ \\
\hline
\end{tabular}

${ }^{20} \ddagger$ Reporting standard not met (too few cases).

Note: Changes in sample size and stratification were implemented following November 2002 Youth Poll.

Source: Department of Defense Polls, JAMRS, 2001-2004 (Question: FPP10E). 


\section{Marine Corps Active Duty Propensity}

TABLE 5-1. Youth Marine Corps active duty propensity: $2001-2004^{21}$

Male and Female (age 16-21)

Percent (\%)

\begin{tabular}{lcccc}
\cline { 2 - 4 } Year & Definitely & Probably & Probably Not & Definitely Not \\
\hline Youth Poll 1 (Apr 01) & 1.3 & 4.7 & 22.7 & 71.2 \\
Youth Poll 2 (Aug 01) & .9 & 4.6 & 24.8 & 69.5 \\
Youth Poll 3 (Nov 01) & 1.2 & 6.9 & 28.0 & 63.9 \\
Youth Poll 4 (Nov 02) & 1.1 & 7.2 & 25.2 & 66.5 \\
Youth Poll 5 (June 03) & 1.8 & 6.7 & 28.0 & 63.4 \\
Youth Poll 6 (Nov 03) & 1.8 & 7.7 & 25.8 & 64.7 \\
Youth Poll 7 (May 04) & 1.8 & 5.6 & 29.3 & 63.2 \\
Youth Poll 8 (Nov 04) & 1.6 & 6.1 & 27.8 & 64.5 \\
\hline
\end{tabular}

Male (age 16-21)

Percent (\%)

\begin{tabular}{lcccc}
\cline { 2 - 4 } Year & Definitely & Probably & Probably Not & Definitely Not \\
\hline Youth Poll 1 (Apr 01) & 2.3 & 7.1 & 29.8 & 60.8 \\
Youth Poll 2 (Aug 01) & 1.5 & 6.8 & 30.8 & 60.9 \\
Youth Poll 3 (Nov 01) & 1.7 & 10.2 & 35.5 & 52.6 \\
Youth Poll 4 (Nov 02) & 2.0 & 10.6 & 31.5 & 55.9 \\
Youth Poll 5 (June 03) & 2.6 & 9.0 & 35.0 & 53.1 \\
Youth Poll 6 (Nov 03) & 3.3 & 10.7 & 32.3 & 53.7 \\
Youth Poll 7 (May 04) & 3.0 & 7.6 & 36.3 & 53.0 \\
Youth Poll 8 (Nov 04) & 2.6 & 9.5 & 34.6 & 53.3 \\
\hline
\end{tabular}

Female (age 16-21)

Percent (\%)

\begin{tabular}{lcccc}
\cline { 2 - 4 } Year & Definitely & Probably & Probably Not & Definitely Not \\
\hline Youth Poll 1 (Apr 01) & .3 & 2.5 & 16.3 & 80.7 \\
Youth Poll 2 (Aug 01) & .4 & 2.6 & 19.2 & 77.6 \\
Youth Poll 3 (Nov 01) & .7 & 3.8 & 21.0 & 74.5 \\
Youth Poll 4 (Nov 02) & .2 & 3.9 & 19.1 & 76.8 \\
Youth Poll 5 (June 03) & 1.0 & 4.2 & 20.8 & 74.0 \\
Youth Poll 6 (Nov 03) & .3 & 4.7 & 19.2 & 75.8 \\
Youth Poll 7 (May 04) & .7 & 3.4 & 22.1 & 73.7 \\
Youth Poll 8 (Nov 04) & .6 & 2.6 & 20.9 & 75.9 \\
\hline
\end{tabular}

${ }^{21} \ddagger$ Reporting standard not met (too few cases).

Note: Changes in sample size and stratification were implemented following November 2002 Youth Poll.

Source: Department of Defense Polls, JAMRS, 2001-2004 (Question: FPP10D). 


\section{Marine Corps Active Duty Propensity}

TABLE 5-2. Youth Marine Corps active duty propensity, by race/ethnicity: $2001-2004^{22}$

\begin{tabular}{|c|c|c|c|c|}
\hline \multirow{3}{*}{$\begin{array}{l}\text { Male and Female (age 16-21) } \\
\text { (definitely \& probably) } \\
\text { Year }\end{array}$} & \multicolumn{4}{|c|}{ Percent (\%) } \\
\hline & \multicolumn{4}{|c|}{ Race/ethnicity } \\
\hline & Total & White & Black & Hispanic \\
\hline Youth Poll 1 (Apr 01) & 6.0 & 4.2 & 7.9 & 11.4 \\
\hline Youth Poll 2 (Aug 01) & 5.5 & 3.4 & 6.6 & 11.5 \\
\hline Youth Poll 3 (Nov 01) & 8.1 & 6.2 & 10.7 & 12.0 \\
\hline Youth Poll 4 (Nov 02) & 8.2 & 5.3 & 10.3 & 15.7 \\
\hline Youth Poll 5 (June 03) & 8.5 & 5.6 & 11.2 & 16.9 \\
\hline Youth Poll 6 (Nov 03) & 9.5 & 6.9 & 10.4 & 17.2 \\
\hline Youth Poll 7 (May 04) & 7.4 & 4.5 & 8.7 & 17.1 \\
\hline Youth Poll 8 (Nov 04) & 7.7 & 5.0 & 7.0 & 16.4 \\
\hline Male (age 16-21) & \multicolumn{4}{|c|}{ Percent (\%) } \\
\hline (definitely \& probably) & \multicolumn{4}{|c|}{ Race/ethnicity } \\
\hline Year & Total & White & Black & Hispanic \\
\hline Youth Poll 1 (Apr 01) & 9.4 & 7.1 & 13.6 & 15.7 \\
\hline Youth Poll 2 (Aug 01) & 8.2 & 5.1 & 12.6 & 16.3 \\
\hline Youth Poll 3 (Nov 01) & 11.9 & 10.1 & 16.2 & 14.4 \\
\hline Youth Poll 4 (Nov 02) & 12.6 & 9.1 & 16.3 & 21.6 \\
\hline Youth Poll 5 (June 03) & 11.7 & 8.5 & 15.7 & 21.2 \\
\hline Youth Poll 6 (Nov 03) & 14.0 & 11.0 & 13.9 & 24.9 \\
\hline Youth Poll 7 (May 04) & 10.6 & 6.5 & 12.7 & 23.7 \\
\hline Youth Poll 8 (Nov 04) & 12.0 & 8.3 & 11.3 & 24.9 \\
\hline Female (age 16-21) & \multicolumn{4}{|c|}{ Percent (\%) } \\
\hline (definitely \& probably) & \multicolumn{4}{|c|}{ Race/ethnicity } \\
\hline Year & Total & White & Black & Hispanic \\
\hline Youth Poll 1 (Apr 01) & 2.8 & 1.2 & 4.1 & 7.7 \\
\hline Youth Poll 2 (Aug 01) & 3.0 & 1.7 & 2.9 & 6.3 \\
\hline Youth Poll 3 (Nov 01) & 4.5 & 2.2 & 7.6 & 9.4 \\
\hline Youth Poll 4 (Nov 02) & 4.1 & 1.6 & 4.3 & 11.1 \\
\hline Youth Poll 5 (June 03) & 5.2 & 2.6 & 7.6 & 12.4 \\
\hline Youth Poll 6 (Nov 03) & 5.0 & 2.5 & 7.5 & 9.9 \\
\hline Youth Poll 7 (May 04) & 4.1 & 2.2 & 5.2 & 10.4 \\
\hline Youth Poll 8 (Nov 04) & 3.2 & 1.3 & 4.1 & 7.6 \\
\hline
\end{tabular}

\footnotetext{
${ }^{22}$ Due to relatively small sample sizes, American Indian, Alaska Native, Asian, and Pacific Islander are included in the total but are not shown separately. Black includes African American, and Hispanic includes Latino. Race categories exclude Hispanic origin unless specified otherwise.

‡Reporting standard not met (too few cases).

Note: Changes in sample size and stratification were implemented following November 2002 Youth Poll.

Source: Department of Defense Polls, JAMRS, 2001-2004 (Question: FPP10D).
} 


\section{Marine Corps Active Duty Propensity}

JAMRS

TABLE 5-3. Youth Marine Corps active duty propensity, by age: $2001-2004^{23}$

\begin{tabular}{lccccccc} 
Male and Female (age 16-21) & \multicolumn{7}{c}{ Percent (\%) } \\
\cline { 2 - 7 } $\begin{array}{l}\text { (definitely \& probably) } \\
\text { Year }\end{array}$ & $\mathbf{1 6}$ & $\mathbf{1 7}$ & $\mathbf{1 8}$ & $\mathbf{1 9}$ & $\mathbf{2 0}$ & $\mathbf{2 1}$ \\
\hline & 12.0 & 8.3 & 6.1 & 3.9 & 1.8 & 3.7 \\
\hline Youth Poll 1 (Apr 01) & 12.1 & 5.7 & 6.6 & 3.2 & 3.7 & 1.6 \\
Youth Poll 2 (Aug 01) & 9.3 & 9.7 & 6.8 & 6.7 & 8.7 & 7.3 \\
Youth Poll 3 (Nov 01) & 11.3 & 10.3 & 6.5 & 7.0 & 9.7 & 4.3 \\
Youth Poll 4 (Nov 02) & 13.4 & 8.6 & 10.3 & 6.9 & 5.6 & 5.4 \\
Youth Poll 5 (June 03) & 15.8 & 11.1 & 10.7 & 4.4 & 5.3 & 8.8 \\
Youth Poll 6 (Nov 03) & 11.2 & 9.0 & 5.8 & 5.8 & 4.6 & 7.3 \\
Youth Poll 7 (May 04) & 11.6 & 9.7 & 7.0 & 6.2 & 7.8 & 2.9 \\
Youth Poll 8 (Nov 04) & & & & & & \\
\hline
\end{tabular}

Male (age 16-21)

(definitely \& probably)

\section{Year}

Youth Poll 1 (Apr 01)

Youth Poll 2 (Aug 01)

Youth Poll 3 (Nov 01)

Youth Poll 4 (Nov 02)

Youth Poll 5 (June 03)

Youth Poll 6 (Nov 03)

Youth Poll 7 (May 04)

Youth Poll 8 (Nov 04)

\begin{tabular}{cccccc}
\multicolumn{7}{c}{ Percent (\%) } \\
\hline $\mathbf{7}$ & Age \\
\hline 16 & $\mathbf{1 7}$ & $\mathbf{1 8}$ & $\mathbf{1 9}$ & $\mathbf{2 0}$ & $\mathbf{2 1}$ \\
\hline 15.6 & 12.2 & 11.6 & 5.0 & 3.9 & 7.2 \\
16.6 & 7.0 & 11.1 & 3.6 & 6.1 & 2.7 \\
13.2 & 13.1 & 9.2 & 10.5 & 12.7 & 12.8 \\
13.5 & 17.0 & 11.5 & 9.6 & 14.8 & 8.2 \\
17.5 & 11.8 & 13.6 & 10.2 & 7.8 & 8.5 \\
21.2 & 15.7 & 16.4 & 7.7 & 8.3 & 13.2 \\
14.6 & 13.2 & 9.1 & 7.2 & 6.8 & 11.9 \\
16.5 & 14.2 & 11.0 & 10.7 & 13.8 & 5.2 \\
\hline
\end{tabular}

\section{Female (age 16-21)}

(definitely \& probably)

Year

Youth Poll 1 (Apr 01)

Youth Poll 2 (Aug 01)

Youth Poll 3 (Nov 01)

Youth Poll 4 (Nov 02)

Youth Poll 5 (June 03)

Youth Poll 6 (Nov 03)

Youth Poll 7 (May 04)

Youth Poll 8 (Nov 04)

\begin{tabular}{cccccc}
\multicolumn{7}{c}{ Percent (\%) } \\
\hline $\mathbf{7}$ & Age \\
\hline 16 & $\mathbf{1 7}$ & $\mathbf{1 8}$ & $\mathbf{1 9}$ & $\mathbf{2 0}$ & $\mathbf{2 1}$ \\
\hline 8.5 & 3.7 & 1.2 & 2.7 & 0.0 & 1.4 \\
6.4 & 4.2 & 2.6 & 2.9 & 1.7 & .8 \\
5.7 & 5.9 & 4.3 & 3.5 & 5.4 & 2.1 \\
8.8 & 4.1 & 1.4 & 4.7 & 4.3 & 1.2 \\
9.3 & 5.2 & 6.9 & 3.5 & 3.2 & 2.5 \\
10.4 & 6.0 & 5.1 & 1.0 & 2.5 & 4.5 \\
7.7 & 4.8 & 2.1 & 4.4 & 2.2 & 2.7 \\
6.4 & 5.2 & 2.8 & 1.6 & 1.7 & .6 \\
\hline
\end{tabular}

${ }^{23} \neq$ Reporting standard not met (too few cases).

Note: Changes in sample size and stratification were implemented following November 2002 Youth Poll.

Source: Department of Defense Polls, JAMRS, 2001-2004 (Question: FPP10D).

Page A-23 DoD May 2004 Youth Poll 


\section{Marine Corps Active Duty Propensity}

TABLE 5-4. Youth Marine Corps active duty propensity, by geographic region: $2001-2004^{24}$

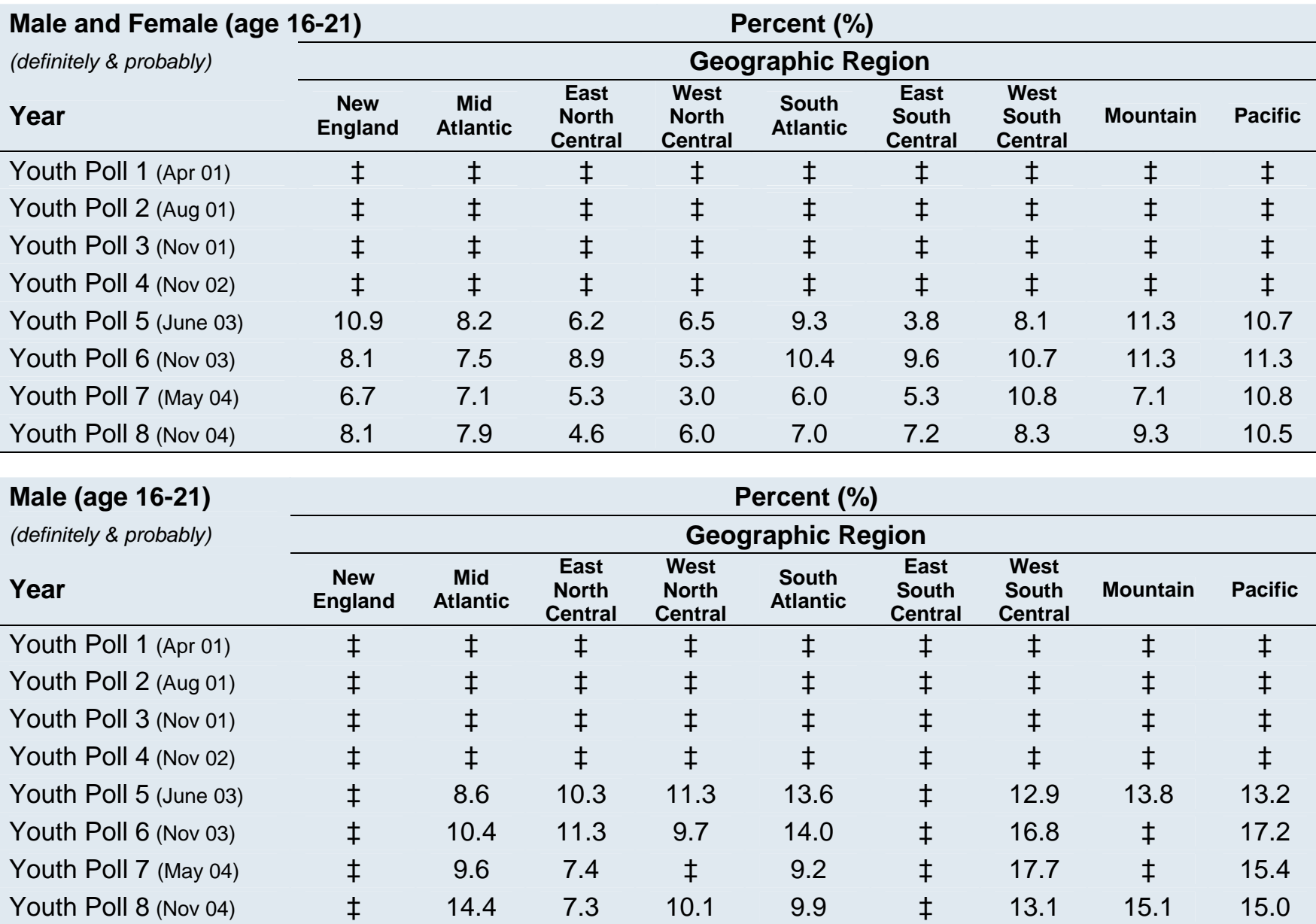

\begin{tabular}{|c|c|c|c|c|c|c|c|c|c|}
\hline \multirow{3}{*}{$\begin{array}{l}\text { Female (age 16-21) } \\
\text { (definitely \& probably) } \\
\text { Year }\end{array}$} & \multicolumn{9}{|c|}{ Percent (\%) } \\
\hline & \multicolumn{9}{|c|}{ Geographic Region } \\
\hline & $\begin{array}{l}\text { New } \\
\text { England }\end{array}$ & $\begin{array}{c}\text { Mid } \\
\text { Atlantic }\end{array}$ & $\begin{array}{c}\text { East } \\
\text { North } \\
\text { Central }\end{array}$ & $\begin{array}{c}\text { West } \\
\text { North } \\
\text { Central }\end{array}$ & $\begin{array}{c}\text { South } \\
\text { Atlantic }\end{array}$ & $\begin{array}{c}\text { East } \\
\text { South } \\
\text { Central }\end{array}$ & $\begin{array}{l}\text { West } \\
\text { South } \\
\text { Central }\end{array}$ & Mountain & Pacific \\
\hline Youth Poll 1 (Apr 01) & $\ddagger$ & $\ddagger$ & $\ddagger$ & $\ddagger$ & $\ddagger$ & $\ddagger$ & $\ddagger$ & $\ddagger$ & $\ddagger$ \\
\hline Youth Poll 2 (Aug 01) & $\ddagger$ & $\ddagger$ & $\ddagger$ & $\ddagger$ & $\ddagger$ & $\ddagger$ & $\ddagger$ & $\ddagger$ & $\ddagger$ \\
\hline Youth Poll 3 (Nov 01) & $\ddagger$ & $\ddagger$ & $\ddagger$ & $\ddagger$ & $\ddagger$ & $\ddagger$ & $\ddagger$ & $\ddagger$ & $\ddagger$ \\
\hline Youth Poll 4 (Nov 02) & $\ddagger$ & $\ddagger$ & $\ddagger$ & $\ddagger$ & $\ddagger$ & $\ddagger$ & $\ddagger$ & $\ddagger$ & $\ddagger$ \\
\hline Youth Poll 5 (June 03) & $\ddagger$ & 7.7 & 3.1 & 2.0 & 4.8 & $\ddagger$ & 4.0 & 8.3 & 7.9 \\
\hline Youth Poll 6 (Nov 03) & $\ddagger$ & 4.7 & 6.3 & .9 & 6.7 & 2.8 & 4.8 & 7.8 & 5.4 \\
\hline Youth Poll 7 (May 04) & $\ddagger$ & 4.0 & 3.2 & $\ddagger$ & 3.0 & $\ddagger$ & 4.6 & $\ddagger$ & 6.3 \\
\hline Youth Poll 8 (Nov 04) & $\ddagger$ & 1.3 & 2.2 & 6 & 4.3 & $\ddagger$ & 4.3 & 3.6 & 5.1 \\
\hline
\end{tabular}

${ }^{24} \ddagger$ Reporting standard not met (too few cases).

Note: Changes in sample size and stratification were implemented following November 2002 Youth Poll.

Source: Department of Defense Polls, JAMRS, 2001-2004 (Question: FPP10D).

Page A-24 DoD May 2004 Youth Poll 


\section{Marine Corps Active Duty Propensity}

JAMRS

TABLE 5-5. Youth Marine Corps active duty propensity, by high school grades: $2001-2004^{25}$

\begin{tabular}{|c|c|c|c|c|c|c|c|}
\hline \multicolumn{4}{|c|}{ Male and Female (age 16-21) } & \multicolumn{4}{|c|}{ Percent (\%) } \\
\hline \multirow{2}{*}{$\begin{array}{l}\text { (definitely \& probably) } \\
\text { Year }\end{array}$} & \multicolumn{7}{|c|}{ High School Grades } \\
\hline & $\begin{array}{l}\text { Mostly } \\
\text { A's }\end{array}$ & $\begin{array}{l}\text { Mostly A's } \\
\& \text { B's }\end{array}$ & $\begin{array}{l}\text { Mostly } \\
\text { B's }\end{array}$ & $\begin{array}{l}\text { Mostly B's } \\
\& \text { C's }\end{array}$ & $\begin{array}{l}\text { Mostly } \\
\text { C's }\end{array}$ & $\begin{array}{c}\text { Mostly C's } \\
\text { \& D's }\end{array}$ & $\begin{array}{l}\text { Mostly D's } \\
\text { and Lower }\end{array}$ \\
\hline Youth Poll 1 (Apr 01) & 1.1 & 4.7 & 5.7 & 7.5 & $\ddagger$ & 9.0 & $\ddagger$ \\
\hline Youth Poll 2 (Aug 01) & 4.1 & 3.2 & 3.9 & 8.6 & $\ddagger$ & 7.8 & $\ddagger$ \\
\hline Youth Poll 3 (Nov 01) & 2.6 & 5.6 & 9.5 & 12.4 & $\ddagger$ & $\ddagger$ & $\ddagger$ \\
\hline Youth Poll 4 (Nov 02) & 3.7 & 4.5 & 5.0 & 14.4 & 13.4 & $\ddagger$ & $\ddagger$ \\
\hline Youth Poll 5 (June 03) & 3.3 & 7.1 & 5.8 & 11.6 & 12.7 & 20.2 & $\ddagger$ \\
\hline Youth Poll 6 (Nov 03) & 4.6 & 7.8 & 6.9 & 13.8 & 6.8 & 20.4 & $\ddagger$ \\
\hline Youth Poll 7 (May 04) & 3.2 & 5.3 & 5.9 & 9.8 & 13.4 & 14.8 & $\ddagger$ \\
\hline Youth Poll 8 (Nov 04) & 3.9 & 5.5 & 5.8 & 11.7 & 13.8 & 15.3 & $\ddagger$ \\
\hline Male (age $16-21$ ) & \multicolumn{7}{|c|}{ Percent (\%) } \\
\hline (definitely \& probably) & \multicolumn{7}{|c|}{ High School Grades } \\
\hline Year & $\begin{array}{l}\text { Mostly } \\
\text { A's }\end{array}$ & $\begin{array}{l}\text { Mostly A's } \\
\text { \& B's }\end{array}$ & $\begin{array}{l}\text { Mostly } \\
\text { B's }\end{array}$ & $\begin{array}{l}\text { Mostly B's } \\
\text { \& C's }\end{array}$ & $\begin{array}{l}\text { Mostly } \\
\text { C's }\end{array}$ & $\begin{array}{l}\text { Mostly C's } \\
\text { \& D's }\end{array}$ & $\begin{array}{l}\text { Mostly D's } \\
\text { and Lower }\end{array}$ \\
\hline Youth Poll 1 (Apr 01) & $\ddagger$ & 7.0 & $\ddagger$ & 9.9 & $\ddagger$ & $\ddagger$ & $\ddagger$ \\
\hline Youth Poll 2 (Aug 01) & $\ddagger$ & 4.2 & 5.6 & 14.0 & $\ddagger$ & $\ddagger$ & $\ddagger$ \\
\hline Youth Poll 3 (Nov 01) & 2.0 & 7.4 & 15.6 & 17.3 & $\ddagger$ & $\ddagger$ & $\ddagger$ \\
\hline Youth Poll 4 (Nov 02) & $\ddagger$ & 7.0 & 6.6 & 18.5 & $\ddagger$ & $\ddagger$ & $\ddagger$ \\
\hline Youth Poll 5 (June 03) & 4.9 & 10.1 & 7.9 & 15.3 & 13.7 & 20.3 & $\ddagger$ \\
\hline Youth Poll 6 (Nov 03) & 6.3 & 12.1 & 8.5 & 19.7 & 8.9 & 26.1 & $\ddagger$ \\
\hline Youth Poll 7 (May 04) & 4.2 & 8.6 & 6.8 & 12.9 & $\ddagger$ & 21.1 & $\ddagger$ \\
\hline Youth Poll 8 (Nov 04) & 7.4 & 8.1 & 9.0 & 17.2 & 19.7 & $\ddagger$ & $\ddagger$ \\
\hline
\end{tabular}

Females (age 16-21)

(definitely \& probably)

\section{Year}

Youth Poll 1 (Apr 01)

Youth Poll 2 (Aug 01)

Youth Poll 3 (Nov 01)

Youth Poll 4 (Nov 02)

Youth Poll 5 (June 03)

Youth Poll 6 (Nov 03)

Youth Poll 7 (May 04)

Youth Poll 8 (Nov 04) 


\section{Air Force Active Duty Propensity}

TABLE 6-1. Youth Air Force active duty propensity: $2001-2004^{26}$

Male and Female (age 16-21)

Percent (\%)

\begin{tabular}{lcccc}
\cline { 2 - 4 } Year & Definitely & Probably & Probably Not & Definitely Not \\
\hline Youth Poll 1 (Apr 01) & 1.5 & 7.4 & 23.3 & 67.6 \\
Youth Poll 2 (Aug 01) & 1.5 & 5.9 & 26.3 & 66.2 \\
Youth Poll 3 (Nov 01) & 1.8 & 7.4 & 30.9 & 59.9 \\
Youth Poll 4 (Nov 02) & 1.9 & 8.3 & 26.8 & 63.0 \\
Youth Poll 5 (June 03) & 2.1 & 7.9 & 28.5 & 61.3 \\
Youth Poll 6 (Nov 03) & 1.7 & 9.3 & 27.6 & 61.5 \\
Youth Poll 7 (May 04) & 2.2 & 9.3 & 29.3 & 59.1 \\
Youth Poll 8 (Nov 04) & 2.3 & 8.0 & 28.8 & 60.9 \\
\hline
\end{tabular}

Male (age 16-21)

Percent (\%)

\begin{tabular}{lcccc}
\cline { 2 - 4 } Year & Definitely & Probably & Probably Not & Definitely Not \\
\hline Youth Poll 1 (Apr 01) & 2.1 & 10.6 & 29.5 & 57.6 \\
Youth Poll 2 (Aug 01) & 1.6 & 6.5 & 33.8 & 58.0 \\
Youth Poll 3 (Nov 01) & 2.9 & 10.0 & 38.8 & 48.3 \\
Youth Poll 4 (Nov 02) & 2.7 & 10.6 & 31.2 & 55.5 \\
Youth Poll 5 (June 03) & 3.0 & 10.9 & 34.2 & 51.6 \\
Youth Poll 6 (Nov 03) & 2.5 & 10.8 & 35.2 & 51.4 \\
Youth Poll 7 (May 04) & 3.1 & 11.4 & 35.1 & 50.2 \\
Youth Poll 8 (Nov 04) & 3.2 & 10.4 & 35.2 & 51.0 \\
\hline
\end{tabular}

Female (age 16-21)

Percent (\%)

Year

Definitely Probably $\quad$ Probably Not Definitely Not

Youth Poll 1 (Apr 01)

.9

4.5

17.6

76.9

Youth Poll 2 (Aug 01)

1.4

5.4

19.2

74.0

Youth Poll 3 (Nov 01)

.7

5.0

23.5

70.7

Youth Poll 4 (Nov 02)

1.1

6.0

22.6

70.3

Youth Poll 5 (June 03)

1.3

4.8

22.7

71.2

Youth Poll 6 (Nov 03)

.8

7.7

19.9

71.6

Youth Poll 7 (May 04)

1.2

7.1

23.4

68.3

Youth Poll 8 (Nov 04)

1.3

5.6

22.1

70.9

${ }^{26} \ddagger$ Reporting standard not met (too few cases).

Note: Changes in sample size and stratification were implemented following November 2002 Youth Poll.

Source: Department of Defense Polls, JAMRS, 2001-2004 (Question: FPP10C). 


\section{Air Force Active Duty Propensity}

TABLE 6-2. Youth Air Force active duty propensity, by race/ethnicity: $2001-2004^{27}$

\begin{tabular}{|c|c|c|c|c|}
\hline \multirow{3}{*}{$\begin{array}{l}\text { Male and Female (age 16-21) } \\
\text { (definitely \& probably) } \\
\text { Year }\end{array}$} & \multicolumn{4}{|c|}{ Percent (\%) } \\
\hline & \multicolumn{4}{|c|}{ Race/ethnicity } \\
\hline & Total & White & Black & Hispanic \\
\hline Youth Poll 1 (Apr 01) & 8.9 & 6.9 & 12.2 & 13.9 \\
\hline Youth Poll 2 (Aug 01) & 7.4 & 4.7 & 11.6 & 12.3 \\
\hline Youth Poll 3 (Nov 01) & 9.2 & 7.0 & 11.2 & 15.8 \\
\hline Youth Poll 4 (Nov 02) & 10.1 & 7.3 & 12.5 & 19.0 \\
\hline Youth Poll 5 (June 03) & 10.1 & 7.5 & 14.4 & 16.1 \\
\hline Youth Poll 6 (Nov 03) & 10.9 & 7.3 & 16.6 & 17.1 \\
\hline Youth Poll 7 (May 04) & 11.4 & 8.3 & 13.1 & 21.1 \\
\hline Youth Poll 8 (Nov 04) & 10.3 & 7.6 & 12.6 & 16.1 \\
\hline Male (age 16-21) & \multicolumn{4}{|c|}{ Percent (\%) } \\
\hline (definitely \& probably) & \multicolumn{4}{|c|}{ Race/ethnicity } \\
\hline Year & Total & White & Black & Hispanic \\
\hline Youth Poll 1 (Apr 01) & 12.7 & 10.9 & 12.3 & 20.4 \\
\hline Youth Poll 2 (Aug 01) & 8.1 & 5.2 & 16.1 & 11.9 \\
\hline Youth Poll 3 (Nov 01) & 12.9 & 10.3 & 11.3 & 23.5 \\
\hline Youth Poll 4 (Nov 02) & 13.2 & 9.4 & 16.9 & 27.6 \\
\hline Youth Poll 5 (June 03) & 13.9 & 10.1 & 21.2 & 23.0 \\
\hline Youth Poll 6 (Nov 03) & 13.3 & 8.8 & 22.2 & 20.0 \\
\hline Youth Poll 7 (May 04) & 14.5 & 11.2 & 15.2 & 24.4 \\
\hline Youth Poll 8 (Nov 04) & 13.6 & 11.6 & 12.1 & 18.6 \\
\hline Female (age 16-21) & \multicolumn{4}{|c|}{ Percent (\%) } \\
\hline (definitely \& probably) & \multicolumn{4}{|c|}{ Race/ethnicity } \\
\hline Year & Total & White & Black & Hispanic \\
\hline Youth Poll 1 (Apr 01) & 5.4 & 2.9 & 12.2 & 8.3 \\
\hline Youth Poll 2 (Aug 01) & 6.8 & 4.2 & 8.8 & 12.7 \\
\hline Youth Poll 3 (Nov 01) & 5.7 & 3.7 & 11.1 & 7.6 \\
\hline Youth Poll 4 (Nov 02) & 7.1 & 5.3 & 8.2 & 12.3 \\
\hline Youth Poll 5 (June 03) & 6.1 & 4.7 & 8.9 & 8.9 \\
\hline Youth Poll 6 (Nov 03) & 8.5 & 5.7 & 11.8 & 14.3 \\
\hline Youth Poll 7 (May 04) & 8.2 & 5.1 & 11.3 & 17.8 \\
\hline Youth Poll 8 (Nov 04) & 6.9 & 3.2 & 13.0 & 13.4 \\
\hline
\end{tabular}

${ }^{27}$ Due to relatively small sample sizes, American Indian, Alaska Native, Asian, and Pacific Islander are included in the total but are not shown separately. Black includes African American, and Hispanic includes Latino. Race categories exclude Hispanic origin unless specified otherwise.

‡Reporting standard not met (too few cases).

Note: Changes in sample size and stratification were implemented following November 2002 Youth Poll.

Source: Department of Defense Polls, JAMRS, 2001-2004 (Question: FPP10C). 


\section{Air Force Active Duty Propensity}

JAMRS

TABLE 6-3. Youth Air Force active duty propensity, by age: $2001-2004^{28}$

\begin{tabular}{lcccccc} 
Male and Female (age 16-21) & \multicolumn{7}{c}{ Percent (\%) } \\
\cline { 2 - 7 } (definitely \& probably) & $\mathbf{7 9}$ & $\mathbf{1 7}$ & $\mathbf{1 8}$ & $\mathbf{1 9}$ & $\mathbf{2 0}$ & $\mathbf{2 1}$ \\
\cline { 2 - 7 } Year & 13.8 & 11.9 & 7.9 & 7.6 & 5.1 & 7.2 \\
\hline Youth Poll 1 (Apr 01) & 10.9 & 7.7 & 9.3 & 6.4 & 6.3 & 3.9 \\
Youth Poll 2 (Aug 01) & 13.8 & 12.1 & 9.8 & 5.0 & 10.5 & 4.0 \\
Youth Poll 3 (Nov 01) & 12.8 & 11.3 & 11.0 & 8.9 & 10.5 & 6.2 \\
Youth Poll 4 (Nov 02) & 14.4 & 12.4 & 10.0 & 9.8 & 8.4 & 4.9 \\
Youth Poll 5 (June 03) & 16.2 & 12.5 & 12.3 & 5.0 & 11.0 & 7.4 \\
Youth Poll 6 (Nov 03) & 15.7 & 13.5 & 10.7 & 10.9 & 7.1 & 9.9 \\
Youth Poll 7 (May 04) & 15.0 & 11.2 & 11.1 & 8.1 & 7.0 & 8.7 \\
Youth Poll 8 (Nov 04) & & & & & & \\
\hline
\end{tabular}

\begin{tabular}{lcccccc} 
Male (age 16-21) & \multicolumn{7}{c}{ Percent (\%) } \\
\cline { 2 - 7 } (definitely \& probably) & \multicolumn{7}{c}{ Age } \\
\cline { 2 - 7 } Year & 16 & $\mathbf{1 7}$ & $\mathbf{1 8}$ & $\mathbf{1 9}$ & $\mathbf{2 0}$ & $\mathbf{2 1}$ \\
\hline Youth Poll 1 (Apr 01) & 16.4 & 16.6 & 9.5 & 14.0 & 6.6 & 12.1 \\
Youth Poll 2 (Aug 01) & 10.3 & 10.3 & 9.1 & 7.5 & 9.8 & 0.0 \\
Youth Poll 3 (Nov 01) & 18.5 & 15.1 & 11.7 & 9.1 & 15.8 & 7.1 \\
Youth Poll 4 (Nov 02) & 13.7 & 15.2 & 15.5 & 13.4 & 12.8 & 8.2 \\
Youth Poll 5 (June 03) & 17.8 & 14.5 & 13.5 & 16.8 & 12.8 & 7.8 \\
Youth Poll 6 (Nov 03) & 18.7 & 15.2 & 14.0 & 5.7 & 15.9 & 9.0 \\
Youth Poll 7 (May 04) & 20.1 & 16.1 & 14.2 & 13.4 & 10.8 & 11.7 \\
Youth Poll 8 (Nov 04) & 20.0 & 13.2 & 16.6 & 12.7 & 8.8 & 9.6 \\
\hline
\end{tabular}

Female (age 16-21)

(definitely \& probably)

Year

Youth Poll 1 (Apr 01)

Youth Poll 2 (Aug 01)

Youth Poll 3 (Nov 01)

Youth Poll 4 (Nov 02)

Youth Poll 5 (June 03)

Youth Poll 6 (Nov 03)

Youth Poll 7 (May 04)

Youth Poll 8 (Nov 04)

\begin{tabular}{cccccc}
\multicolumn{7}{c}{ Percent (\%) } \\
\hline $\mathbf{7}$ & Age \\
\hline 16 & $\mathbf{1 7}$ & $\mathbf{1 8}$ & $\mathbf{1 9}$ & $\mathbf{2 0}$ & $\mathbf{2 1}$ \\
\hline 11.4 & 6.3 & 6.4 & .9 & 3.9 & 3.9 \\
11.6 & 5.0 & 9.4 & 5.4 & 3.4 & 6.8 \\
9.2 & 8.8 & 8.0 & 1.7 & 6.2 & 1.1 \\
11.9 & 7.6 & 6.3 & 4.9 & 7.9 & 4.6 \\
11.0 & 10.2 & 6.2 & 2.7 & 3.6 & 2.2 \\
13.7 & 9.4 & 10.6 & 4.4 & 6.4 & 5.8 \\
11.3 & 11.0 & 6.8 & 8.4 & 3.2 & 8.0 \\
9.6 & 9.2 & 5.5 & 3.5 & 5.3 & 7.8 \\
\hline
\end{tabular}

${ }^{28} \neq$ Reporting standard not met (too few cases).

Note: Changes in sample size and stratification were implemented following November 2002 Youth Poll.

Source: Department of Defense Polls, JAMRS, 2001-2004 (Question: FPP10C).

Page A-28 DoD May 2004 Youth Poll 


\section{Air Force Active Duty Propensity}

JAMRS

TABLE 6-4. Youth Air Force active duty propensity, by geographic region: $2001-2004^{29}$

\begin{tabular}{|c|c|c|c|c|c|c|c|c|c|}
\hline \multirow{3}{*}{ (definitely \& probably) } & \multicolumn{9}{|c|}{ Percent (\%) } \\
\hline & \multicolumn{9}{|c|}{ Geographic Region } \\
\hline & $\begin{array}{c}\text { New } \\
\text { England }\end{array}$ & $\begin{array}{c}\text { Mid } \\
\text { Atlantic }\end{array}$ & $\begin{array}{c}\text { East } \\
\text { North } \\
\text { Central }\end{array}$ & $\begin{array}{c}\text { West } \\
\text { North } \\
\text { Central }\end{array}$ & $\begin{array}{c}\text { South } \\
\text { Atlantic }\end{array}$ & $\begin{array}{l}\text { East } \\
\text { South } \\
\text { Central }\end{array}$ & $\begin{array}{l}\text { West } \\
\text { South } \\
\text { Central }\end{array}$ & Mountain & Pacific \\
\hline Youth Poll 1 (Apr 01) & $\ddagger$ & $\ddagger$ & $\ddagger$ & $\ddagger$ & $\ddagger$ & $\ddagger$ & $\ddagger$ & $\ddagger$ & $\ddagger$ \\
\hline Youth Poll 2 (Aug 01) & $\ddagger$ & $\ddagger$ & $\ddagger$ & $\ddagger$ & $\ddagger$ & $\ddagger$ & $\ddagger$ & $\ddagger$ & $\neq$ \\
\hline Youth Poll 3 (Nov 01) & $\ddagger$ & $\ddagger$ & $\ddagger$ & $\ddagger$ & $\ddagger$ & $\ddagger$ & $\ddagger$ & $\ddagger$ & $\ddagger$ \\
\hline Youth Poll 4 (Nov 02) & $\ddagger$ & $\ddagger$ & $\ddagger$ & $\ddagger$ & $\ddagger$ & $\ddagger$ & $\ddagger$ & $\ddagger$ & $\ddagger$ \\
\hline Youth Poll 5 (June 03) & 9.7 & 7.4 & 7.5 & 7.7 & 13.2 & 9.0 & 12.4 & 11.8 & 10.6 \\
\hline Youth Poll 6 (Nov 03) & 15.2 & 9.9 & 6.9 & 7.5 & 13.1 & 11.5 & 14.2 & 10.0 & 11.8 \\
\hline Youth Poll 7 (May 04) & 6.6 & 11.3 & 8.5 & 3.0 & 9.0 & 10.5 & 16.1 & 18.4 & 15.0 \\
\hline Youth Poll 8 (Nov 04) & 7.3 & 7.6 & 8.2 & 9.2 & 11.1 & 8.0 & 15.6 & 10.0 & 11.7 \\
\hline \multirow{3}{*}{$\begin{array}{l}\text { Male (age 16-21) } \\
\text { (definitely \& probably) } \\
\text { Year }\end{array}$} & \multicolumn{9}{|c|}{ Percent (\%) } \\
\hline & \multicolumn{9}{|c|}{ Geographic Region } \\
\hline & $\begin{array}{c}\text { New } \\
\text { England }\end{array}$ & $\begin{array}{c}\text { Mid } \\
\text { Atlantic }\end{array}$ & $\begin{array}{c}\text { East } \\
\text { North } \\
\text { Central } \\
\end{array}$ & $\begin{array}{c}\text { West } \\
\text { North } \\
\text { Central } \\
\end{array}$ & $\begin{array}{c}\text { South } \\
\text { Atlantic }\end{array}$ & $\begin{array}{c}\text { East } \\
\text { South } \\
\text { Central } \\
\end{array}$ & $\begin{array}{c}\text { West } \\
\text { South } \\
\text { Central }\end{array}$ & Mountain & Pacific \\
\hline Youth Poll 1 (Apr 01) & $\mp$ & $\ddagger$ & $\ddagger$ & $\ddagger$ & $\ddagger$ & $\ddagger$ & $\ddagger$ & $\ddagger$ & $\ddagger$ \\
\hline Youth Poll 2 (Aug 01) & $\ddagger$ & $\ddagger$ & $\ddagger$ & $\ddagger$ & $\ddagger$ & $\ddagger$ & $\ddagger$ & $\ddagger$ & $\ddagger$ \\
\hline Youth Poll 3 (Nov 01) & $\ddagger$ & $\ddagger$ & $\ddagger$ & $\ddagger$ & $\ddagger$ & $\ddagger$ & $\ddagger$ & $\ddagger$ & $\ddagger$ \\
\hline Youth Poll 4 (Nov 02) & $\ddagger$ & $\ddagger$ & $\ddagger$ & $\ddagger$ & $\ddagger$ & $\ddagger$ & $\ddagger$ & $\ddagger$ & $\ddagger$ \\
\hline Youth Poll 5 (June 03) & $\ddagger$ & 7.8 & 11.1 & 10.3 & 20.9 & $\ddagger$ & 19.7 & 12.0 & 14.1 \\
\hline Youth Poll 6 (Nov 03) & $\ddagger$ & 11.8 & 7.8 & 9.9 & 17.1 & $\ddagger$ & 15.4 & $\ddagger$ & 14.6 \\
\hline Youth Poll 7 (May 04) & $\ddagger$ & 15.5 & 11.8 & $\ddagger$ & 11.0 & $\ddagger$ & 23.8 & $\ddagger$ & 17.5 \\
\hline Youth Poll 8 (Nov 04) & $\ddagger$ & 11.0 & 12.7 & 13.5 & 12.8 & $\ddagger$ & 17.8 & 15.8 & 14.9 \\
\hline
\end{tabular}

Female (age 16-21)

(definitely \& probably)

Year

Youth Poll 1 (Apr 01)

Youth Poll 2 (Aug 01)

Youth Poll 3 (Nov 01)

Youth Poll 4 (Nov 02)

Youth Poll 5 (June 03)

Youth Poll 6 (Nov 03)

Youth Poll 7 (May 04)

Youth Poll 8 (Nov 04)
Percent (\%)

\section{Geographic Region}

North South South South Mountain Pacific

Central Atlantic Central Central

$\begin{array}{ll}\ddagger & \ddagger \\ \ddagger & \ddagger \\ \ddagger & \ddagger \\ \ddagger & \ddagger\end{array}$

$\ddagger \quad \ddagger$

$\ddagger \quad \ddagger$

$\ddagger \quad \ddagger$

$\ddagger \quad \ddagger$

$\ddagger \quad \ddagger$

$\ddagger \quad \ddagger$

$\ddagger$

6.1

11.5

6.5

$\ddagger \quad 6.9$

4.7

5.2

5.1

$\ddagger$

13.0

8.7

9.0

6.3

5.2

$\ddagger$

7.0

9.3

$\ddagger$

12.5

9.6

$\ddagger$

13.7

4.2

7.9

\footnotetext{
${ }^{29} \ddagger$ Reporting standard not met (too few cases).

Note: Changes in sample size and stratification were implemented following November 2002 Youth Poll.

Source: Department of Defense Polls, JAMRS, 2001-2004 (Question: FPP10C).
} 


\section{Air Force Active Duty Propensity}

JAMRS

TABLE 6-5. Youth Air Force active duty propensity, by high school grades: $2001-2004^{30}$

\begin{tabular}{|c|c|c|c|c|c|c|c|}
\hline \multicolumn{4}{|c|}{ Male and Female (age 16-21) } & \multicolumn{4}{|c|}{ Percent (\%) } \\
\hline \multirow{2}{*}{$\begin{array}{l}\text { (definitely \& probably) } \\
\text { Year }\end{array}$} & \multicolumn{7}{|c|}{ High School Grades } \\
\hline & $\begin{array}{l}\text { Mostly } \\
\text { A's }\end{array}$ & $\begin{array}{l}\text { Mostly A's } \\
\text { \& B's }\end{array}$ & $\begin{array}{l}\text { Mostly } \\
\text { B's }\end{array}$ & $\begin{array}{l}\text { Mostly B's } \\
\text { \& C's }\end{array}$ & $\begin{array}{l}\text { Mostly } \\
\text { C's }\end{array}$ & $\begin{array}{l}\text { Mostly C's } \\
\text { \& D's }\end{array}$ & $\begin{array}{l}\text { Mostly D's } \\
\text { and Lower }\end{array}$ \\
\hline Youth Poll 1 (Apr 01) & 7.6 & 5.7 & 8.4 & 11.9 & $\ddagger$ & 7.9 & $\ddagger$ \\
\hline Youth Poll 2 (Aug 01) & 4.9 & 7.3 & 5.1 & 9.7 & $\ddagger$ & 6.3 & $\ddagger$ \\
\hline Youth Poll 3 (Nov 01) & 4.1 & 6.8 & 11.0 & 14.3 & $\ddagger$ & $\ddagger$ & $\ddagger$ \\
\hline Youth Poll 4 (Nov 02) & 6.1 & 7.3 & 9.8 & 12.9 & 20.1 & $\ddagger$ & $\ddagger$ \\
\hline Youth Poll 5 (June 03) & 5.0 & 9.7 & 7.8 & 14.4 & 9.6 & 15.4 & $\ddagger$ \\
\hline Youth Poll 6 (Nov 03) & 6.3 & 10.4 & 13.9 & 13.3 & 10.5 & 12.3 & $\ddagger$ \\
\hline Youth Poll 7 (May 04) & 6.5 & 11.1 & 13.9 & 13.6 & 10.5 & 15.5 & $\ddagger$ \\
\hline Youth Poll 8 (Nov 04) & 4.2 & 9.0 & 7.2 & 14.5 & 13.1 & 18.2 & $\ddagger$ \\
\hline \multirow{3}{*}{$\begin{array}{l}\text { Male (age 16-21) } \\
\text { (definitely \& probably) } \\
\text { Year }\end{array}$} & \multicolumn{7}{|c|}{ Percent (\%) } \\
\hline & \multicolumn{7}{|c|}{ High School Grades } \\
\hline & $\begin{array}{l}\text { Mostly } \\
\text { A's }\end{array}$ & $\begin{array}{l}\text { Mostly A's } \\
\text { \& B's }\end{array}$ & $\begin{array}{l}\text { Mostly } \\
\text { B's }\end{array}$ & $\begin{array}{l}\text { Mostly B's } \\
\text { \& C's }\end{array}$ & $\begin{array}{l}\text { Mostly } \\
\text { C's }\end{array}$ & $\begin{array}{l}\text { Mostly C's } \\
\text { \& D's }\end{array}$ & $\begin{array}{l}\text { Mostly D's } \\
\text { and Lower }\end{array}$ \\
\hline Youth Poll 1 (Apr 01) & $\ddagger$ & 6.9 & $\ddagger$ & 16.6 & $\ddagger$ & $\ddagger$ & $\ddagger$ \\
\hline Youth Poll 2 (Aug 01) & $\ddagger$ & 6.4 & 4.3 & 11.9 & $\ddagger$ & $\ddagger$ & $\ddagger$ \\
\hline Youth Poll 3 (Nov 01) & 5.7 & 10.8 & 15.7 & 17.1 & $\ddagger$ & $\ddagger$ & $\ddagger$ \\
\hline Youth Poll 4 (Nov 02) & $\ddagger$ & 8.8 & 10.9 & 14.9 & $\ddagger$ & $\ddagger$ & $\ddagger$ \\
\hline Youth Poll 5 (June 03) & 7.5 & 13.4 & 11.7 & 19.3 & 9.4 & 17.3 & $\ddagger$ \\
\hline Youth Poll 6 (Nov 03) & 8.7 & 14.2 & 16.1 & 16.5 & 7.4 & 13.7 & $\ddagger$ \\
\hline Youth Poll 7 (May 04) & 9.8 & 12.5 & 18.1 & 17.4 & $\ddagger$ & 20.2 & $\ddagger$ \\
\hline Youth Poll 8 (Nov 04) & 6.6 & 10.8 & 9.7 & 19.2 & 15.8 & $\ddagger$ & $\ddagger$ \\
\hline
\end{tabular}

Females (age 16-21)

(definitely \& probably)

\section{Year}

Youth Poll 1 (Apr 01)

Youth Poll 2 (Aug 01)

Youth Poll 3 (Nov 01)

Youth Poll 4 (Nov 02)

Youth Poll 5 (June 03)

Youth Poll 6 (Nov 03)

Youth Poll 7 (May 04)

Youth Poll 8 (Nov 04)
Mostly

\& B's

Percent (\%) 


\section{Coast Guard Active Duty Propensity}

TABLE 7-1. Youth Coast Guard active duty propensity: $2001-2004^{31}$

Male and Female (age 16-21)

Percent (\%)

\begin{tabular}{lcccc}
\cline { 2 - 4 } Year & Definitely & Probably & Probably Not & Definitely Not \\
\hline Youth Poll 1 (Apr 01) & .2 & 3.6 & 23.2 & 72.7 \\
Youth Poll 2 (Aug 01) & .7 & 4.7 & 25.7 & 68.8 \\
Youth Poll 3 (Nov 01) & .8 & 4.8 & 31.4 & 62.8 \\
Youth Poll 4 (Nov 02) & .7 & 5.0 & 26.8 & 67.2 \\
Youth Poll 5 (June 03) & .6 & 5.2 & 29.2 & 64.8 \\
Youth Poll 6 (Nov 03) & .4 & 5.5 & 29.8 & 64.2 \\
Youth Poll 7 (May 04) & .5 & 5.8 & 31.3 & 62.3 \\
Youth Poll 8 (Nov 04) & .9 & 4.9 & 30.0 & 64.0 \\
\hline
\end{tabular}

Male (age 16-21)

Percent (\%)

\begin{tabular}{lcccc}
\cline { 2 - 4 } Year & Definitely & Probably & Probably Not & Definitely Not \\
\hline Youth Poll 1 (Apr 01) & .3 & 5.8 & 29.2 & 64.7 \\
Youth Poll 2 (Aug 01) & 1.2 & 6.1 & 31.1 & 61.6 \\
Youth Poll 3 (Nov 01) & .8 & 6.4 & 39.2 & 53.5 \\
Youth Poll 4 (Nov 02) & .9 & 5.8 & 31.6 & 61.4 \\
Youth Poll 5 (June 03) & 1.0 & 6.4 & 35.0 & 57.4 \\
Youth Poll 6 (Nov 03) & .7 & 7.7 & 36.4 & 55.2 \\
Youth Poll 7 (May 04) & .8 & 7.7 & 38.4 & 53.0 \\
Youth Poll 8 (Nov 04) & 1.2 & 6.1 & 36.9 & 55.6 \\
\hline
\end{tabular}

Female (age 16-21)

Percent (\%)

\begin{tabular}{lcccc}
\cline { 2 - 4 } Year & Definitely & Probably & Probably Not & Definitely Not \\
\hline Youth Poll 1 (Apr 01) & .2 & 1.6 & 17.7 & 80.2 \\
Youth Poll 2 (Aug 01) & .2 & 3.4 & 20.6 & 75.5 \\
Youth Poll 3 (Nov 01) & .7 & 3.4 & 24.1 & 71.6 \\
Youth Poll 4 (Nov 02) & .6 & 4.2 & 22.2 & 72.8 \\
Youth Poll 5 (June 03) & .3 & 4.0 & 23.2 & 72.4 \\
Youth Poll 6 (Nov 03) & .1 & 3.3 & 23.2 & 73.3 \\
Youth Poll 7 (May 04) & .3 & 3.8 & 24.0 & 71.8 \\
Youth Poll 8 (Nov 04) & .6 & 3.8 & 22.9 & 72.6
\end{tabular}

${ }^{31} \neq$ Reporting standard not met (too few cases).

Note: Changes in sample size and stratification were implemented following November 2002 Youth Poll.

Source: Department of Defense Polls, JAMRS, 2001-2004 (Question: FPP10A). 


\section{Coast Guard Active Duty Propensity}

TABLE 7-2. Youth Coast Guard active duty propensity, by race/ethnicity: $2001-2004^{32}$

\begin{tabular}{|c|c|c|c|c|}
\hline \multirow{3}{*}{$\begin{array}{l}\text { Male and Female (age 16-21) } \\
\text { (definitely \& probably) } \\
\text { Year }\end{array}$} & \multicolumn{4}{|c|}{ Percent (\%) } \\
\hline & \multicolumn{4}{|c|}{ Race/ethnicity } \\
\hline & Total & White & Black & Hispanic \\
\hline Youth Poll 1 (Apr 01) & 3.9 & 2.9 & 5.2 & 6.9 \\
\hline Youth Poll 2 (Aug 01) & 5.4 & 3.0 & 8.6 & 12.1 \\
\hline Youth Poll 3 (Nov 01) & 5.6 & 4.1 & 8.2 & 10.2 \\
\hline Youth Poll 4 (Nov 02) & 5.7 & 4.4 & 7.4 & 9.9 \\
\hline Youth Poll 5 (June 03) & 5.9 & 4.2 & 7.7 & 10.6 \\
\hline Youth Poll 6 (Nov 03) & 5.9 & 3.4 & 8.1 & 12.5 \\
\hline Youth Poll 7 (May 04) & 6.3 & 4.3 & 7.9 & 11.6 \\
\hline Youth Poll 8 (Nov 04) & 5.9 & 3.6 & 8.6 & 10.4 \\
\hline Male (age 16-21) & \multicolumn{4}{|c|}{ Percent (\%) } \\
\hline (definitely \& probably) & \multicolumn{4}{|c|}{ Race/ethnicity } \\
\hline Year & Total & White & Black & Hispanic \\
\hline Youth Poll 1 (Apr 01) & 6.1 & 5.1 & 8.4 & 9.1 \\
\hline Youth Poll 2 (Aug 01) & 7.2 & 4.5 & 10.4 & 15.5 \\
\hline Youth Poll 3 (Nov 01) & 7.2 & 5.2 & 9.5 & 15.2 \\
\hline Youth Poll 4 (Nov 02) & 6.7 & 5.5 & 5.7 & 13.2 \\
\hline Youth Poll 5 (June 03) & 7.4 & 5.5 & 9.0 & 13.7 \\
\hline Youth Poll 6 (Nov 03) & 8.4 & 4.6 & 11.6 & 17.8 \\
\hline Youth Poll 7 (May 04) & 8.5 & 6.0 & 9.8 & 14.8 \\
\hline Youth Poll 8 (Nov 04) & 7.3 & 5.3 & 10.0 & 11.3 \\
\hline Female (age 16-21) & \multicolumn{4}{|c|}{ Percent (\%) } \\
\hline (definitely \& probably) & \multicolumn{4}{|c|}{ Race/ethnicity } \\
\hline Year & Total & White & Black & Hispanic \\
\hline Youth Poll 1 (Apr 01) & 1.8 & .7 & 3.0 & 5.1 \\
\hline Youth Poll 2 (Aug 01) & 3.7 & 1.5 & 7.4 & 8.3 \\
\hline Youth Poll 3 (Nov 01) & 4.1 & 2.9 & 7.4 & 4.9 \\
\hline Youth Poll 4 (Nov 02) & 4.8 & 3.2 & 9.1 & 7.3 \\
\hline Youth Poll 5 (June 03) & 4.3 & 2.9 & 6.6 & 7.3 \\
\hline Youth Poll 6 (Nov 03) & 3.5 & 2.1 & 5.2 & 7.4 \\
\hline Youth Poll 7 (May 04) & 4.1 & 2.3 & 6.3 & 8.3 \\
\hline Youth Poll 8 (Nov 04) & 4.4 & 1.8 & 7.6 & 9.4 \\
\hline
\end{tabular}

\footnotetext{
${ }^{32}$ Due to relatively small sample sizes, American Indian, Alaska Native, Asian, and Pacific Islander are included in the total but are not shown separately. Black includes African American, and Hispanic includes Latino. Race categories exclude Hispanic origin unless specified otherwise.

‡Reporting standard not met (too few cases).

Note: Changes in sample size and stratification were implemented following November 2002 Youth Poll.

Source: Department of Defense Polls, JAMRS, 2001-2004 (Question: FPP10A).
} 


\section{Coast Guard Active Duty Propensity}

JAMRS

TABLE 7-3. Youth Coast Guard active duty propensity, by age: $2001-2004^{33}$

\begin{tabular}{lcccccc} 
Male and Female (age 16-21) & \multicolumn{7}{c}{ Percent (\%) } \\
\cline { 2 - 7 } $\begin{array}{l}\text { (definitely \& probably) } \\
\text { Year }\end{array}$ & $\mathbf{1 6}$ & $\mathbf{1 7}$ & $\mathbf{1 8}$ & $\mathbf{1 9}$ & $\mathbf{2 0}$ & $\mathbf{2 1}$ \\
\hline & 3.3 & 5.0 & 4.3 & 3.8 & 4.3 & 2.4 \\
\hline Youth Poll 1 (Apr 01) & 8.2 & 4.9 & 8.7 & 4.2 & 4.7 & 1.5 \\
Youth Poll 2 (Aug 01) & 6.0 & 6.2 & 6.3 & 3.3 & 5.8 & 6.0 \\
Youth Poll 3 (Nov 01) & 8.5 & 5.5 & 4.9 & 5.3 & 6.1 & 3.8 \\
Youth Poll 4 (Nov 02) & 7.9 & 8.5 & 5.1 & 5.4 & 4.4 & 3.6 \\
Youth Poll 5 (June 03) & 8.9 & 6.6 & 6.7 & 3.1 & 4.4 & 5.3 \\
Youth Poll 6 (Nov 03) & 8.7 & 6.4 & 7.1 & 6.6 & 1.8 & 7.0 \\
Youth Poll 7 (May 04) & 7.5 & 5.6 & 4.6 & 6.0 & 4.9 & 6.4 \\
Youth Poll 8 (Nov 04) & & & & & & \\
\hline
\end{tabular}

Male (age 16-21)

(definitely \& probably)

\section{Year}

Youth Poll 1 (Apr 01)

Youth Poll 2 (Aug 01)

Youth Poll 3 (Nov 01)

Youth Poll 4 (Nov 02)

Youth Poll 5 (June 03)

Youth Poll 6 (Nov 03)

Youth Poll 7 (May 04)

Youth Poll 8 (Nov 04)

\begin{tabular}{cccccc}
\multicolumn{7}{c}{ Percent (\%) } \\
\hline \multicolumn{7}{c}{ Age } \\
\hline $\mathbf{1 6}$ & $\mathbf{1 7}$ & $\mathbf{1 8}$ & $\mathbf{1 9}$ & $\mathbf{2 0}$ & $\mathbf{2 1}$ \\
\hline 4.2 & 7.5 & 6.3 & 6.0 & 6.6 & 6.0 \\
8.5 & 7.7 & 8.2 & 7.0 & 9.3 & 2.1 \\
9.2 & 6.9 & 8.2 & 2.1 & 9.6 & 7.3 \\
11.8 & 7.3 & 6.8 & 3.4 & 5.2 & 5.3 \\
8.3 & 10.6 & 6.7 & 6.6 & 7.5 & 4.2 \\
10.9 & 8.2 & 9.5 & 6.0 & 8.1 & 7.1 \\
11.9 & 9.8 & 7.2 & 9.1 & 2.6 & 10.1 \\
7.8 & 8.0 & 6.8 & 8.5 & 6.7 & 5.8 \\
\hline
\end{tabular}

\section{Female (age 16-21)}

(definitely \& probably)

Year

Youth Poll 1 (Apr 01)

Youth Poll 2 (Aug 01)

Youth Poll 3 (Nov 01)

Youth Poll 4 (Nov 02)

Youth Poll 5 (June 03)

Youth Poll 6 (Nov 03)

Youth Poll 7 (May 04)

Youth Poll 8 (Nov 04)

\begin{tabular}{cccccc}
\multicolumn{7}{c}{ Percent (\%) } \\
\hline $\mathbf{7}$ & \multicolumn{7}{c}{ Age } \\
\hline 2.5 & 17 & $\mathbf{1 8}$ & $\mathbf{1 9}$ & $\mathbf{2 0}$ & $\mathbf{2 1}$ \\
7.8 & 1.9 & 2.5 & 1.5 & 2.3 & 0.0 \\
2.9 & 2.1 & 9.1 & 1.8 & 1.0 & 1.0 \\
5.0 & 3.4 & 4.4 & 4.4 & 2.8 & 4.9 \\
7.6 & 3.8 & 3.1 & 6.9 & 6.9 & 2.7 \\
7.0 & 4.2 & 3.3 & 4.2 & 1.0 & 3.0 \\
5.4 & 3.1 & 4.0 & .1 & .9 & 3.5 \\
7.2 & 3.0 & 2.3 & 4.2 & .9 & 4.0 \\
& & & 3.4 & 3.0 & 7.1 \\
\hline
\end{tabular}

${ }^{33} \neq$ Reporting standard not met (too few cases).

Note: Changes in sample size and stratification were implemented following November 2002 Youth Poll.

Source: Department of Defense Polls, JAMRS, 2001-2004 (Question: FPP10A). 


\section{Coast Guard Active Duty Propensity}

TABLE 7-4. Youth Coast Guard active duty propensity, by geographic region: $2001-2004^{34}$

\begin{tabular}{|c|c|c|c|c|c|c|c|c|c|}
\hline Male and Female (age & $6-21)$ & & & & rcent ( $\%$ & & & & \\
\hline (definitely \& probably) & & & & Geo & raphic $R$ & gion & & & \\
\hline  & $\begin{array}{l}\text { New } \\
\text { England }\end{array}$ & $\begin{array}{c}\text { Mid } \\
\text { Atlantic }\end{array}$ & $\begin{array}{c}\text { East } \\
\text { North } \\
\text { Central } \\
\end{array}$ & $\begin{array}{c}\text { West } \\
\text { North } \\
\text { Central }\end{array}$ & $\begin{array}{c}\text { South } \\
\text { Atlantic }\end{array}$ & $\begin{array}{c}\text { East } \\
\text { South } \\
\text { Central }\end{array}$ & $\begin{array}{c}\text { West } \\
\text { South } \\
\text { Central }\end{array}$ & Mountain & Pacific \\
\hline Youth Poll 1 (Apr 01) & $\ddagger$ & $\ddagger$ & $\ddagger$ & $\neq$ & $\ddagger$ & $\ddagger$ & $\ddagger$ & $\ddagger$ & $\ddagger$ \\
\hline Youth Poll 2 (Aug 01) & $\ddagger$ & $\ddagger$ & $\ddagger$ & $\ddagger$ & $\ddagger$ & $\ddagger$ & $\ddagger$ & $\ddagger$ & $\ddagger$ \\
\hline Youth Poll 3 (Nov 01) & $\ddagger$ & $\ddagger$ & $\ddagger$ & $\ddagger$ & $\ddagger$ & $\ddagger$ & $\ddagger$ & $\ddagger$ & $\ddagger$ \\
\hline Youth Poll 4 (Nov 02) & $\ddagger$ & $\ddagger$ & $\ddagger$ & $\ddagger$ & $\ddagger$ & $\ddagger$ & $\ddagger$ & $\ddagger$ & $\ddagger$ \\
\hline Youth Poll 5 (June 03) & 6.8 & 7.8 & 3.7 & 1.5 & 7.6 & 5.2 & 5.3 & 4.3 & 8.1 \\
\hline Youth Poll 6 (Nov 03) & 3.5 & 5.7 & 3.6 & 4.2 & 6.9 & 6.1 & 7.9 & 3.4 & 8.4 \\
\hline Youth Poll 7 (May 04) & 3.1 & 5.6 & 6.0 & 1.4 & 6.9 & 3.7 & 6.7 & 11.8 & 7.7 \\
\hline Youth Poll 8 (Nov 04) & 3.7 & 3.0 & 2.7 & 2.0 & 8.8 & 6.6 & 10.7 & 4.9 & 7.1 \\
\hline Male (age 16-21) & & & & & ercent $(9$ & & & & \\
\hline (definitely \& probably) & & & & Geo & aphic $\mathrm{R}$ & gion & & & \\
\hline Year & $\begin{array}{c}\text { New } \\
\text { England }\end{array}$ & $\begin{array}{c}\text { Mid } \\
\text { Atlantic } \\
\end{array}$ & $\begin{array}{c}\text { East } \\
\text { North } \\
\text { Central } \\
\end{array}$ & $\begin{array}{c}\text { West } \\
\text { North } \\
\text { Central } \\
\end{array}$ & $\begin{array}{c}\text { South } \\
\text { Atlantic }\end{array}$ & $\begin{array}{c}\text { East } \\
\text { South } \\
\text { Central } \\
\end{array}$ & $\begin{array}{c}\text { West } \\
\text { South } \\
\text { Central } \\
\end{array}$ & Mountain & Pacific \\
\hline Youth Poll 1 (Apr 01) & $\ddagger$ & $\ddagger$ & $\ddagger$ & $\ddagger$ & $\ddagger$ & $\ddagger$ & $\ddagger$ & $\ddagger$ & $\ddagger$ \\
\hline Youth Poll 2 (Aug 01) & $\ddagger$ & $\ddagger$ & $\ddagger$ & $\ddagger$ & $\ddagger$ & $\ddagger$ & $\ddagger$ & $\ddagger$ & $\ddagger$ \\
\hline Youth Poll 3 (Nov 01) & $\ddagger$ & $\ddagger$ & $\ddagger$ & $\ddagger$ & $\ddagger$ & $\ddagger$ & $\ddagger$ & $\ddagger$ & $\ddagger$ \\
\hline Youth Poll 4 (Nov 02) & $\ddagger$ & $\ddagger$ & $\ddagger$ & $\ddagger$ & $\ddagger$ & $\ddagger$ & $\ddagger$ & $\ddagger$ & $\ddagger$ \\
\hline Youth Poll 5 (June 03) & $\ddagger$ & 7.5 & 5.3 & 3.2 & 10.5 & $\ddagger$ & 7.1 & 2.7 & 10.4 \\
\hline Youth Poll 6 (Nov 03) & $\ddagger$ & 7.3 & 4.0 & 8.3 & 9.6 & $\ddagger$ & 11.1 & $\ddagger$ & 12.1 \\
\hline Youth Poll 7 (May 04) & $\ddagger$ & 6.6 & 7.9 & $\ddagger$ & 8.3 & $\ddagger$ & 10.6 & $\ddagger$ & 11.6 \\
\hline Youth Poll 8 (Nov 04) & $\ddagger$ & 3.9 & 2.3 & 3.0 & 13.3 & $\ddagger$ & 11.6 & 5.0 & 9.0 \\
\hline Female (age 16-21) & & & & & ercent $(9$ & & & & \\
\hline (definitely \& probably) & & & & Geo & raphic $\mathbf{R}$ & gion & & & \\
\hline Year & $\begin{array}{c}\text { New } \\
\text { England }\end{array}$ & $\begin{array}{c}\text { Mid } \\
\text { Atlantic } \\
\end{array}$ & $\begin{array}{c}\text { East } \\
\text { North } \\
\text { Central } \\
\end{array}$ & $\begin{array}{c}\text { West } \\
\text { North } \\
\text { Central } \\
\end{array}$ & $\begin{array}{c}\text { South } \\
\text { Atlantic }\end{array}$ & $\begin{array}{c}\text { East } \\
\text { South } \\
\text { Central } \\
\end{array}$ & $\begin{array}{c}\text { West } \\
\text { South } \\
\text { Central } \\
\end{array}$ & Mountain & Pacific \\
\hline Youth Poll 1 (Apr 01) & $\ddagger$ & $\ddagger$ & $\ddagger$ & $\mp$ & $\mp$ & $\ddagger$ & $\ddagger$ & $\ddagger$ & $\ddagger$ \\
\hline Youth Poll 2 (Aug 01) & $\ddagger$ & $\ddagger$ & $\ddagger$ & $\ddagger$ & $\ddagger$ & $\ddagger$ & $\ddagger$ & $\ddagger$ & $\ddagger$ \\
\hline Youth Poll 3 (Nov 01) & $\ddagger$ & $\ddagger$ & $\ddagger$ & $\ddagger$ & $\ddagger$ & $\ddagger$ & $\ddagger$ & $\ddagger$ & $\ddagger$ \\
\hline Youth Poll 4 (Nov 02) & $\ddagger$ & $\ddagger$ & $\ddagger$ & $\ddagger$ & $\ddagger$ & $\ddagger$ & $\ddagger$ & $\ddagger$ & $\ddagger$ \\
\hline Youth Poll 5 (June 03) & $\ddagger$ & 8.1 & 2.5 & 0.0 & 4.6 & $\ddagger$ & 3.7 & 6.3 & 5.3 \\
\hline Youth Poll 6 (Nov 03) & $\ddagger$ & 4.0 & 3.0 & 0.0 & 4.3 & 3.2 & 4.9 & 1.8 & 4.6 \\
\hline Youth Poll 7 (May 04) & $\ddagger$ & 4.3 & 4.2 & $\ddagger$ & 5.7 & $\ddagger$ & 3.2 & $\ddagger$ & 3.8 \\
\hline Youth Poll 8 (Nov 04) & $\ddagger$ & 2.1 & 3.1 & .8 & 4.8 & $\ddagger$ & 10.0 & 4.8 & 4.7 \\
\hline
\end{tabular}

${ }^{34}$ ‡Reporting standard not met (too few cases).

Note: Changes in sample size and stratification were implemented following November 2002 Youth Poll.

Source: Department of Defense Polls, JAMRS, 2001-2004 (Question: FPP10A).

Page A-34 DoD May 2004 Youth Poll 


\section{Coast Guard Active Duty Propensity}

TABLE 7-5. Youth Coast Guard active duty propensity, by high school grades: $2001-2004^{35}$



Females (age 16-21)

(definitely \& probably)

Year

Youth Poll 1 (Apr 01)

Youth Poll 2 (Aug 01)

Youth Poll 3 (Nov 01)

Youth Poll 4 (Nov 02)

Youth Poll 5 (June 03)

Youth Poll 6 (Nov 03)

Youth Poll 7 (May 04)

Youth Poll 8 (Nov 04)
Percent (\%)

\section{High School Grades}

\begin{tabular}{ccccccc}
$\begin{array}{c}\text { Mostly } \\
\text { A's }\end{array}$ & $\begin{array}{c}\text { Mostly A's } \\
\text { \& B's }\end{array}$ & $\begin{array}{c}\text { Mostly } \\
\text { B's }\end{array}$ & $\begin{array}{c}\text { Mostly B's } \\
\text { \& C's }\end{array}$ & $\begin{array}{c}\text { Mostly } \\
\text { C's }\end{array}$ & $\begin{array}{c}\text { Mostly C's } \\
\text { \& D's }\end{array}$ & $\begin{array}{c}\text { Mostly D's } \\
\text { and Lower }\end{array}$ \\
\hline 0.0 & 1.8 & $\ddagger$ & 2.8 & $\ddagger$ & $\ddagger$ & $\ddagger$ \\
2.7 & 2.3 & 3.2 & 5.3 & $\ddagger$ & $\ddagger$ & $\ddagger$ \\
1.7 & 3.9 & 1.6 & 7.9 & $\ddagger$ & $\ddagger$ & $\ddagger$ \\
1.3 & 4.1 & 6.3 & 8.4 & $\ddagger$ & $\ddagger$ & $\ddagger$ \\
2.4 & 4.8 & 3.8 & 4.7 & $\ddagger$ & $\ddagger$ & $\ddagger$ \\
.7 & 3.9 & 4.7 & 5.0 & $\ddagger$ & $\ddagger$ & $\ddagger$ \\
1.2 & 4.6 & 2.9 & 6.5 & $\ddagger$ & $\ddagger$ & $\ddagger$ \\
2.2 & 4.7 & 2.2 & 6.7 & $\ddagger$ & $\ddagger$ & $\ddagger$ \\
\hline
\end{tabular}

${ }^{35}$ ‡Reporting standard not met (too few cases).

Note: Changes in sample size and stratification were implemented following November 2002 Youth Poll.

Source: Department of Defense Polls, JAMRS, 2001-2004 (Question: FPP10A). 


\section{Composite Reserve Propensity}

TABLE 8-1. Youth composite Reserve propensity: $2001-2004^{36}$

Male and Female (age 16-21)

Percent (\%)

\begin{tabular}{lcccc}
\cline { 2 - 4 } Year & Definitely & Probably & Probably Not & Definitely Not \\
\hline Youth Poll 1 (Apr 01) & 1.5 & 11.3 & 29.0 & 58.2 \\
Youth Poll 2 (Aug 01) & 1.7 & 10.9 & 31.7 & 55.6 \\
Youth Poll 3 (Nov 01) & 1.5 & 14.0 & 35.8 & 48.7 \\
Youth Poll 4 (Nov 02) & 1.0 & 12.8 & 32.2 & 53.9 \\
Youth Poll 5 (June 03) & 1.8 & 11.9 & 34.0 & 52.2 \\
Youth Poll 6 (Nov 03) & 1.7 & 14.8 & 31.3 & 52.2 \\
Youth Poll 7 (May 04) & 1.7 & 13.1 & 33.3 & 51.8 \\
Youth Poll 8 (Nov 04) & 1.5 & 12.9 & 32.2 & 53.4 \\
\hline
\end{tabular}

Male (age 16-21)

Percent (\%)

\begin{tabular}{lcccc}
\cline { 2 - 4 } Year & Definitely & Probably & Probably Not & Definitely Not \\
\hline Youth Poll 1 (Apr 01) & 1.7 & 15.3 & 36.0 & 47.0 \\
Youth Poll 2 (Aug 01) & 1.9 & 12.7 & 38.5 & 46.9 \\
Youth Poll 3 (Nov 01) & 1.6 & 19.5 & 42.1 & 36.8 \\
Youth Poll 4 (Nov 02) & 1.2 & 18.4 & 34.9 & 45.5 \\
Youth Poll 5 (June 03) & 2.3 & 16.1 & 39.6 & 41.8 \\
Youth Poll 6 (Nov 03) & 2.1 & 19.5 & 37.1 & 41.3 \\
Youth Poll 7 (May 04) & 2.1 & 16.7 & 40.7 & 40.5 \\
Youth Poll 8 (Nov 04) & 2.2 & 18.1 & 37.7 & 42.0 \\
\hline
\end{tabular}

Female (age 16-21)

Percent (\%)

Year

Definitely Probably $\quad$ Probably Not Definitely Not

Youth Poll 1 (Apr 01)

1.4

7.6

22.4

68.6

Youth Poll 2 (Aug 01)

1.5

9.3

25.4

63.8

Youth Poll 3 (Nov 01)

1.5

8.9

29.9

59.8

Youth Poll 4 (Nov 02)

.8

7.5

29.7

62.0

Youth Poll 5 (June 03)

1.2

7.6

28.4

62.8

Youth Poll 6 (Nov 03)

1.2

10.2

25.4

63.2

Youth Poll 7 (May 04)

1.3

9.4

25.7

63.5

Youth Poll 8 (Nov 04)

.8

7.5

26.7

65.0

\footnotetext{
${ }^{36}$ ‡Reporting standard not met (too few cases).

Note: Changes in sample size and stratification were implemented following November 2002 Youth Poll.

Source: Department of Defense Polls, JAMRS, 2001-2004 (Question: Composite of FPP11 and FPP12).
} 


\section{Composite Reserve Propensity}

JAMRS

TABLE 8-2. Youth composite Reserve propensity, by racelethnicity: $2001-2004^{37}$

\begin{tabular}{|c|c|c|c|c|}
\hline \multirow{3}{*}{$\begin{array}{l}\text { Male and Female (age 16-21) } \\
\text { (definitely \& probably) } \\
\text { Year }\end{array}$} & \multicolumn{4}{|c|}{ Percent (\%) } \\
\hline & \multicolumn{4}{|c|}{ Race/ethnicity } \\
\hline & Total & White & Black & Hispanic \\
\hline Youth Poll 1 (Apr 01) & 12.8 & 9.4 & 18.0 & 21.8 \\
\hline Youth Poll 2 (Aug 01) & 12.7 & 7.1 & 23.5 & 22.9 \\
\hline Youth Poll 3 (Nov 01) & 15.5 & 11.6 & 19.6 & 27.1 \\
\hline Youth Poll 4 (Nov 02) & 13.8 & 9.8 & 19.6 & 21.9 \\
\hline Youth Poll 5 (June 03) & 13.7 & 10.2 & 17.3 & 24.0 \\
\hline Youth Poll 6 (Nov 03) & 16.5 & 11.8 & 25.2 & 25.7 \\
\hline Youth Poll 7 (May 04) & 14.8 & 10.2 & 20.7 & 26.8 \\
\hline Youth Poll 8 (Nov 04) & 14.3 & 11.3 & 15.8 & 22.0 \\
\hline Male (age 16-21) & \multicolumn{4}{|c|}{ Percent (\%) } \\
\hline (definitely \& probably) & \multicolumn{4}{|c|}{ Race/ethnicity } \\
\hline Year & Total & White & Black & Hispanic \\
\hline Youth Poll 1 (Apr 01) & 17.0 & 14.6 & 21.1 & 23.7 \\
\hline Youth Poll 2 (Aug 01) & 14.7 & 9.4 & 27.6 & 24.4 \\
\hline Youth Poll 3 (Nov 01) & 21.1 & 16.8 & 20.2 & 37.3 \\
\hline Youth Poll 4 (Nov 02) & 19.6 & 15.0 & 28.3 & 31.3 \\
\hline Youth Poll 5 (June 03) & 18.5 & 14.3 & 23.8 & 30.4 \\
\hline Youth Poll 6 (Nov 03) & 21.6 & 17.4 & 32.1 & 31.3 \\
\hline Youth Poll 7 (May 04) & 18.8 & 13.2 & 24.3 & 34.0 \\
\hline Youth Poll 8 (Nov 04) & 20.2 & 18.1 & 18.9 & 26.2 \\
\hline Female (age 16-21) & \multicolumn{4}{|c|}{ Percent (\%) } \\
\hline (definitely \& probably) & \multicolumn{4}{|c|}{ Race/ethnicity } \\
\hline Year & Total & White & Black & Hispanic \\
\hline Youth Poll 1 (Apr 01) & 9.0 & 4.3 & 15.9 & 20.2 \\
\hline Youth Poll 2 (Aug 01) & 10.8 & 4.8 & 20.9 & 21.2 \\
\hline Youth Poll 3 (Nov 01) & 10.3 & 6.4 & 19.3 & 16.3 \\
\hline Youth Poll 4 (Nov 02) & 8.3 & 4.7 & 11.1 & 14.6 \\
\hline Youth Poll 5 (June 03) & 8.8 & 5.9 & 12.0 & 17.2 \\
\hline Youth Poll 6 (Nov 03) & 11.4 & 5.9 & 19.4 & 20.3 \\
\hline Youth Poll 7 (May 04) & 10.7 & 6.9 & 17.6 & 19.5 \\
\hline Youth Poll 8 (Nov 04) & 8.3 & 3.9 & 13.7 & 17.7 \\
\hline
\end{tabular}

\footnotetext{
${ }^{37}$ Due to relatively small sample sizes, American Indian, Alaska Native, Asian, and Pacific Islander are included in the total but are not shown separately. Black includes African American, and Hispanic includes Latino. Race categories exclude Hispanic origin unless specified otherwise.

‡Reporting standard not met (too few cases).

Note: Changes in sample size and stratification were implemented following November 2002 Youth Poll.

Source: Department of Defense Polls, JAMRS, 2001-2004 (Question: Composite of FPP11 and FPP12).
} 


\section{Composite Reserve Propensity}

JAMRS

TABLE 8-3. Youth composite Reserve propensity, by age: $2001-2004^{38}$

\begin{tabular}{lcccccc} 
Male and Female (age 16-21) & \multicolumn{7}{c}{ Percent (\%) } \\
\cline { 2 - 7 } $\begin{array}{l}\text { (definitely \& probably) } \\
\text { Year }\end{array}$ & $\mathbf{1 6}$ & $\mathbf{1 7}$ & $\mathbf{1 8}$ & $\mathbf{1 9}$ & $\mathbf{2 0}$ & $\mathbf{2 1}$ \\
\cline { 2 - 7 } & 18.1 & 16.4 & 11.7 & 7.2 & 9.7 & 13.9 \\
\hline Youth Poll 1 (Apr 01) & 18.6 & 13.5 & 14.0 & 9.8 & 10.1 & 9.6 \\
Youth Poll 2 (Aug 01) & 18.0 & 19.4 & 15.9 & 12.3 & 17.1 & 10.4 \\
Youth Poll 3 (Nov 01) & 18.9 & 14.6 & 13.6 & 9.7 & 16.0 & 10.1 \\
Youth Poll 4 (Nov 02) & 18.8 & 16.7 & 12.8 & 13.4 & 10.0 & 10.0 \\
Youth Poll 5 (June 03) & 22.0 & 18.7 & 17.0 & 11.4 & 15.8 & 12.9 \\
Youth Poll 6 (Nov 03) & 20.3 & 16.2 & 15.1 & 12.9 & 10.0 & 13.3 \\
Youth Poll 7 (May 04) & 18.4 & 16.0 & 14.1 & 13.0 & 14.4 & 9.3 \\
Youth Poll 8 (Nov 04) & & & & & 7.0 & \\
\hline
\end{tabular}

\begin{tabular}{lcccccc} 
Male (age 16-21) & \multicolumn{7}{c}{ Percent (\%) } \\
\cline { 2 - 7 } $\begin{array}{l}\text { (definitely \& probably) } \\
\text { Year }\end{array}$ & $\mathbf{1 6}$ & $\mathbf{1 7}$ & $\mathbf{1 8}$ & $\mathbf{1 9}$ & $\mathbf{2 0}$ & $\mathbf{2 1}$ \\
\hline & 22.4 & 20.3 & 15.4 & 10.7 & 12.0 & 21.7 \\
\hline Youth Poll 1 (Apr 01) & 24.0 & 17.2 & 13.8 & 10.3 & 12.7 & 6.9 \\
Youth Poll 2 (Aug 01) & 24.8 & 24.8 & 21.1 & 17.7 & 23.4 & 14.4 \\
Youth Poll 3 (Nov 01) & 21.9 & 20.0 & 23.8 & 14.7 & 20.9 & 15.5 \\
Youth Poll 4 (Nov 02) & 23.9 & 21.1 & 18.4 & 18.7 & 14.4 & 13.7 \\
Youth Poll 5 (June 03) & 27.3 & 23.8 & 22.6 & 14.5 & 22.9 & 16.8 \\
Youth Poll 6 (Nov 03) & 25.3 & 20.0 & 19.5 & 15.1 & 14.8 & 16.6 \\
Youth Poll 7 (May 04) & 25.1 & 19.7 & 22.7 & 19.5 & 23.5 & 10.4 \\
Youth Poll 8 (Nov 04) & & & & & & \\
\hline
\end{tabular}

\begin{tabular}{lcccccc} 
Female (age 16-21) & \multicolumn{7}{c}{ Percent (\%) } \\
\cline { 2 - 7 } (definitely \& probably) & \multicolumn{7}{c}{ Age } \\
\cline { 2 - 7 } Year & 14.0 & 11.8 & $\mathbf{1 7}$ & $\mathbf{1 9}$ & $\mathbf{2 0}$ & $\mathbf{2 1}$ \\
\hline Youth Poll 1 (Apr 01) & 12.1 & 9.6 & 14.2 & 9.4 & 7.9 & 11.7 \\
Youth Poll 2 (Aug 01) & 11.6 & 13.5 & 10.5 & 7.9 & 12.1 & 6.5 \\
Youth Poll 3 (Nov 01) & 15.6 & 9.5 & 3.0 & 5.3 & 10.8 & 5.8 \\
Youth Poll 4 (Nov 02) & 13.6 & 12.1 & 6.9 & 8.1 & 5.1 & 6.5 \\
Youth Poll 5 (June 03) & 16.7 & 13.1 & 11.4 & 8.2 & 9.1 & 9.1 \\
Youth Poll 6 (Nov 03) & 15.2 & 12.5 & 10.0 & 10.6 & 5.1 & 10.0 \\
Youth Poll 7 (May 04) & 11.3 & 12.2 & 5.2 & 6.6 & 5.2 & 8.1 \\
Youth Poll 8 (Nov 04) & & & & & 7.2 & \\
\hline
\end{tabular}

\footnotetext{
${ }^{38}$ ‡Reporting standard not met (too few cases).

Note: Changes in sample size and stratification were implemented following November 2002 Youth Poll. Source: Department of Defense Polls, JAMRS, 2001-2004 (Question: Composite of FPP11 and FPP12).
} 


\section{Composite Reserve Propensity}

JAMRS

TABLE 8-4. Youth composite Reserve propensity, by geographic region: $2001-2004^{39}$



${ }^{39}$ ‡Reporting standard not met (too few cases).

Note: Changes in sample size and stratification were implemented following November 2002 Youth Poll.

Source: Department of Defense Polls, JAMRS, 2001-2004 (Question: Composite of FPP11 and FPP12).

Page A-39 DoD May 2004 Youth Poll 


\section{Composite Reserve Propensity}

JAMRS

TABLE 8-5. Youth composite Reserve propensity, by high school grades: $2001-2004^{40}$

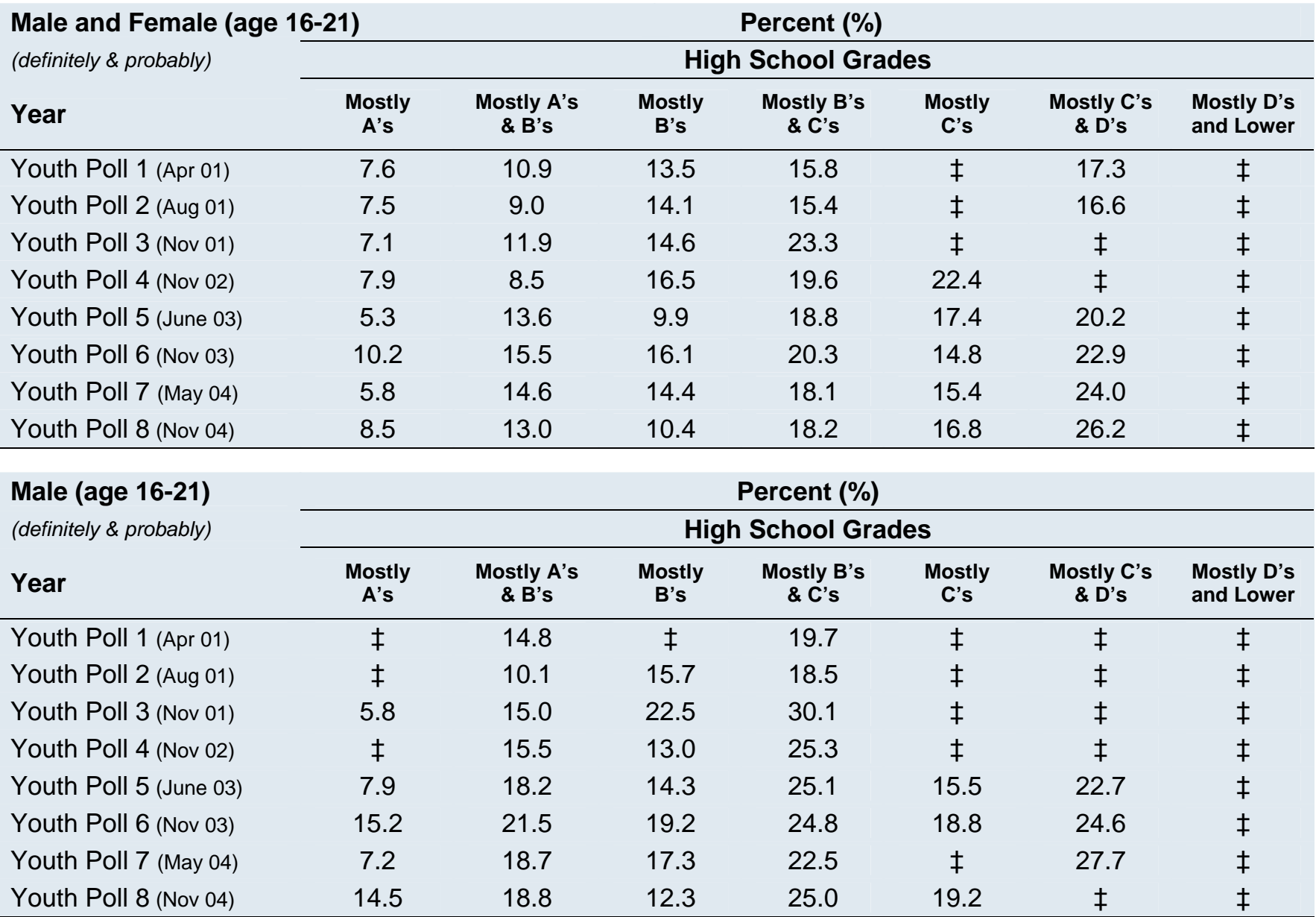

Females (age 16-21)

(definitely \& probably)

\section{Year}

Youth Poll 1 (Apr 01)

Youth Poll 2 (Aug 01)

Youth Poll 3 (Nov 01)

Youth Poll 4 (Nov 02)

Youth Poll 5 (June 03)

Youth Poll 6 (Nov 03)

Youth Poll 7 (May 04)

Youth Poll 8 (Nov 04)

Percent (\%)

\section{High School Grades}




\section{Reserve Propensity}

TABLE 9-1. Youth Reserve propensity: $2001-2004^{41}$

Male and Female (age 16-21)

Percent (\%)

\begin{tabular}{lcccc}
\cline { 2 - 4 } Year & Definitely & Probably & Probably Not & Definitely Not \\
\hline Youth Poll 1 (Apr 01) & 1.1 & 9.4 & 28.1 & 61.3 \\
Youth Poll 2 (Aug 01) & 1.1 & 9.2 & 29.8 & 59.7 \\
Youth Poll 3 (Nov 01) & 1.1 & 11.0 & 34.7 & 53.0 \\
Youth Poll 4 (Nov 02) & .7 & 10.1 & 31.7 & 57.3 \\
Youth Poll 5 (June 03) & 1.2 & 9.6 & 33.7 & 55.3 \\
Youth Poll 6 (Nov 03) & 1.2 & 12.1 & 30.8 & 55.8 \\
Youth Poll 7 (May 04) & 1.3 & 10.2 & 32.6 & 55.6 \\
Youth Poll 8 (Nov 04) & 1.2 & 9.6 & 31.2 & 57.8 \\
\hline
\end{tabular}

Male (age 16-21)

Percent (\%)

\begin{tabular}{lcccc}
\cline { 2 - 4 } Year & Definitely & Probably & Probably Not & Definitely Not \\
\hline Youth Poll 1 (Apr 01) & 1.1 & 12.5 & 35.4 & 51.0 \\
Youth Poll 2 (Aug 01) & 1.1 & 10.6 & 37.2 & 51.0 \\
Youth Poll 3 (Nov 01) & 1.3 & 15.8 & 40.4 & 42.2 \\
Youth Poll 4 (Nov 02) & 1.0 & 14.4 & 36.4 & 47.7 \\
Youth Poll 5 (June 03) & 1.7 & 12.4 & 40.5 & 45.0 \\
Youth Poll 6 (Nov 03) & 1.6 & 16.0 & 37.3 & 45.0 \\
Youth Poll 7 (May 04) & 1.6 & 13.4 & 40.2 & 44.6 \\
Youth Poll 8 (Nov 04) & 1.9 & 13.5 & 37.7 & 46.6 \\
\hline
\end{tabular}

Female (age 16-21)

Percent (\%)

Year

Definitely Probably $\quad$ Probably Not Definitely Not

Youth Poll 1 (Apr 01)

1.1

6.4

21.4

70.9

Youth Poll 2 (Aug 01)

1.1

8.0

22.8

67.8

Youth Poll 3 (Nov 01)

1.0

6.4

29.4

63.1

Youth Poll 4 (Nov 02)

.4

6.0

27.0

66.5

Youth Poll 5 (June 03)

.7

6.6

26.7

65.9

Youth Poll 6 (Nov 03)

.8

8.1

24.2

66.7

Youth Poll 7 (May 04)

1.0

7.0

24.9

66.9

Youth Poll 8 (Nov 04)

.5

5.6

24.6

69.2

\footnotetext{
${ }^{41}$ ‡Reporting standard not met (too few cases).

Note: Changes in sample size and stratification were implemented following November 2002 Youth Poll.

Source: Department of Defense Polls, JAMRS, 2001-2004 (Question: FPP12).
} 


\section{Reserve Propensity}

JAMRS

TABLE 9-2. Youth Reserve propensity, by race/ethnicity: $2001-2004^{42}$

\begin{tabular}{|c|c|c|c|c|}
\hline \multirow{3}{*}{$\begin{array}{l}\text { Male and Female (age 16-21) } \\
\text { (definitely \& probably) } \\
\text { Year }\end{array}$} & \multicolumn{4}{|c|}{ Percent (\%) } \\
\hline & \multicolumn{4}{|c|}{ Race/ethnicity } \\
\hline & Total & White & Black & Hispanic \\
\hline Youth Poll 1 (Apr 01) & 10.5 & 7.9 & 16.2 & 15.6 \\
\hline Youth Poll 2 (Aug 01) & 10.3 & 5.6 & 18.8 & 20.0 \\
\hline Youth Poll 3 (Nov 01) & 12.1 & 8.7 & 15.7 & 22.5 \\
\hline Youth Poll 4 (Nov 02) & 10.8 & 8.1 & 12.1 & 19.1 \\
\hline Youth Poll 5 (June 03) & 10.8 & 7.8 & 14.0 & 20.2 \\
\hline Youth Poll 6 (Nov 03) & 13.3 & 9.5 & 21.4 & 20.0 \\
\hline Youth Poll 7 (May 04) & 11.5 & 8.1 & 13.7 & 23.0 \\
\hline Youth Poll 8 (Nov 04) & 10.8 & 8.4 & 10.8 & 17.8 \\
\hline Male (age 16-21) & \multicolumn{4}{|c|}{ Percent (\%) } \\
\hline (definitely \& probably) & \multicolumn{4}{|c|}{ Race/ethnicity } \\
\hline Year & Total & White & Black & Hispanic \\
\hline Youth Poll 1 (Apr 01) & 13.6 & 12.1 & 16.6 & 17.9 \\
\hline Youth Poll 2 (Aug 01) & 11.7 & 6.9 & 20.7 & 22.1 \\
\hline Youth Poll 3 (Nov 01) & 17.1 & 13.0 & 17.1 & 32.4 \\
\hline Youth Poll 4 (Nov 02) & 15.3 & 12.6 & 16.1 & 26.1 \\
\hline Youth Poll 5 (June 03) & 14.2 & 10.6 & 19.2 & 25.1 \\
\hline Youth Poll 6 (Nov 03) & 17.6 & 14.2 & 27.7 & 25.3 \\
\hline Youth Poll 7 (May 04) & 14.9 & 10.3 & 17.5 & 30.7 \\
\hline Youth Poll 8 (Nov 04) & 15.4 & 13.3 & 13.8 & 21.6 \\
\hline Female (age 16-21) & \multicolumn{4}{|c|}{ Percent (\%) } \\
\hline (definitely \& probably) & \multicolumn{4}{|c|}{ Race/ethnicity } \\
\hline Year & Total & White & Black & Hispanic \\
\hline Youth Poll 1 (Apr 01) & 7.6 & 3.8 & 15.9 & 13.7 \\
\hline Youth Poll 2 (Aug 01) & 9.0 & 4.3 & 17.6 & 17.8 \\
\hline Youth Poll 3 (Nov 01) & 7.4 & 4.3 & 14.9 & 11.9 \\
\hline Youth Poll 4 (Nov 02) & 6.4 & 3.5 & 8.2 & 13.5 \\
\hline Youth Poll 5 (June 03) & 7.3 & 4.7 & 9.6 & 14.9 \\
\hline Youth Poll 6 (Nov 03) & 8.9 & 4.6 & 16.1 & 15.0 \\
\hline Youth Poll 7 (May 04) & 8.0 & 5.7 & 10.3 & 15.3 \\
\hline Youth Poll 8 (Nov 04) & 6.1 & 2.9 & 8.6 & 14.0 \\
\hline
\end{tabular}

${ }^{42}$ Due to relatively small sample sizes, American Indian, Alaska Native, Asian, and Pacific Islander are included in the total but are not shown separately. Black includes African American, and Hispanic includes Latino. Race categories exclude Hispanic origin unless specified otherwise.

‡Reporting standard not met (too few cases).

Note: Changes in sample size and stratification were implemented following November 2002 Youth Poll.

Source: Department of Defense Polls, JAMRS, 2001-2004 (Question: FPP12). 


\section{Reserve Propensity}

JAMRS

TABLE 9-3. Youth Reserve propensity, by age: $2001-2004^{43}$

\begin{tabular}{lcccccc} 
Male and Female (age 16-21) & \multicolumn{7}{c}{ Percent (\%) } \\
\cline { 2 - 7 } (definitely \& probably) & $\mathbf{7 0}$ & $\mathbf{1 7}$ & $\mathbf{1 8}$ & $\mathbf{1 9}$ & $\mathbf{2 0}$ & $\mathbf{2 1}$ \\
\cline { 2 - 7 } Year & 13.8 & 13.9 & 9.1 & 5.5 & 9.2 & 11.5 \\
\hline Youth Poll 1 (Apr 01) & 15.4 & 10.7 & 11.9 & 7.6 & 7.8 & 8.0 \\
Youth Poll 2 (Aug 01) & 14.3 & 15.0 & 13.1 & 7.7 & 12.8 & 9.8 \\
Youth Poll 3 (Nov 01) & 13.8 & 10.3 & 11.1 & 8.4 & 13.0 & 8.1 \\
Youth Poll 4 (Nov 02) & 15.0 & 13.1 & 11.4 & 9.1 & 9.2 & 6.2 \\
Youth Poll 5 (June 03) & 17.5 & 14.9 & 13.2 & 8.5 & 14.2 & 10.3 \\
Youth Poll 6 (Nov 03) & 15.8 & 12.7 & 12.3 & 8.3 & 8.5 & 10.5 \\
Youth Poll 7 (May 04) & 14.0 & 12.8 & 12.0 & 10.2 & 8.3 & 7.0 \\
Youth Poll 8 (Nov 04) & & & & & \multicolumn{7}{c}{ Age } \\
\hline
\end{tabular}

\section{Male (age 16-21)}

(definitely \& probably)

\section{Year}

Youth Poll 1 (Apr 01)

Youth Poll 2 (Aug 01)

Youth Poll 3 (Nov 01)

Youth Poll 4 (Nov 02)

Youth Poll 5 (June 03)

Youth Poll 6 (Nov 03)

Youth Poll 7 (May 04)

Youth Poll 8 (Nov 04)

\begin{tabular}{cccccc}
\multicolumn{7}{c}{ Percent (\%) } \\
\hline $\mathbf{7}$ & Age \\
\hline 16 & $\mathbf{1 7}$ & $\mathbf{1 8}$ & $\mathbf{1 9}$ & $\mathbf{2 0}$ & $\mathbf{2 1}$ \\
\hline 15.6 & 17.0 & 12.4 & 7.4 & 12.0 & 18.4 \\
18.6 & 14.7 & 10.4 & 6.5 & 10.8 & 6.9 \\
20.0 & 20.5 & 18.4 & 12.9 & 17.2 & 13.3 \\
15.6 & 15.5 & 19.4 & 13.7 & 16.1 & 11.0 \\
19.5 & 15.9 & 16.6 & 12.0 & 12.8 & 7.1 \\
22.5 & 18.2 & 18.8 & 11.7 & 21.0 & 12.4 \\
20.4 & 16.6 & 17.1 & 9.1 & 13.1 & 12.0 \\
19.5 & 15.8 & 19.6 & 15.7 & 13.2 & 8.1 \\
\hline
\end{tabular}

\section{Female (age 16-21)}

(definitely \& probably)

\section{Year}

Youth Poll 1 (Apr 01)

Youth Poll 2 (Aug 01)

Youth Poll 3 (Nov 01)

Youth Poll 4 (Nov 02)

Youth Poll 5 (June 03)

Youth Poll 6 (Nov 03)

Youth Poll 7 (May 04)

Youth Poll 8 (Nov 04)

\begin{tabular}{cccccc}
\multicolumn{7}{c}{ Percent (\%) } \\
\hline $\mathbf{7}$ Age \\
\hline 16 & $\mathbf{1 7}$ & $\mathbf{1 8}$ & $\mathbf{1 9}$ & $\mathbf{2 0}$ & $\mathbf{2 1}$ \\
\hline 12.1 & 10.3 & 6.2 & 3.6 & 6.8 & 6.8 \\
11.6 & 6.5 & 13.4 & 8.6 & 5.4 & 8.9 \\
8.8 & 8.9 & 7.7 & 3.5 & 9.3 & 6.5 \\
11.9 & 5.3 & 2.4 & 3.7 & 9.8 & 5.8 \\
10.4 & 10.2 & 6.0 & 6.3 & 5.1 & 5.3 \\
12.4 & 11.2 & 7.8 & 5.2 & 7.9 & 8.2 \\
11.1 & 9.0 & 6.9 & 7.6 & 3.7 & 9.0 \\
8.1 & 9.7 & 4.3 & 4.7 & 3.5 & 5.9 \\
\hline
\end{tabular}

${ }^{43}$ ‡Reporting standard not met (too few cases).

Note: Changes in sample size and stratification were implemented following November 2002 Youth Poll.

Source: Department of Defense Polls, JAMRS, 2001-2004 (Question: FPP12). 


\section{Reserve Propensity}

JAMRS

TABLE 9-4. Youth Reserve propensity, by geographic region: $2001-2004^{44}$

\begin{tabular}{|c|c|c|c|c|c|c|c|c|c|}
\hline Male and Female (age & $6-21)$ & & & & rcent ( $\%$ & & & & \\
\hline (definitely \& probably) & & & & Geo & raphic $R$ & gion & & & \\
\hline Year & $\begin{array}{c}\text { New } \\
\text { England }\end{array}$ & $\begin{array}{c}\text { Mid } \\
\text { Atlantic } \\
\end{array}$ & $\begin{array}{c}\text { East } \\
\text { North } \\
\text { Central }\end{array}$ & $\begin{array}{c}\text { West } \\
\text { North } \\
\text { Central }\end{array}$ & $\begin{array}{c}\text { South } \\
\text { Atlantic }\end{array}$ & $\begin{array}{c}\text { East } \\
\text { South } \\
\text { Central }\end{array}$ & $\begin{array}{c}\text { West } \\
\text { South } \\
\text { Central }\end{array}$ & Mountain & Pacific \\
\hline Youth Poll 1 (Apr 01) & $\ddagger$ & $\ddagger$ & $\ddagger$ & $\ddagger$ & $\ddagger$ & $\ddagger$ & $\ddagger$ & $\ddagger$ & $\ddagger$ \\
\hline Youth Poll 2 (Aug 01) & $\ddagger$ & $\ddagger$ & $\ddagger$ & $\ddagger$ & $\ddagger$ & $\neq$ & $\neq$ & $\neq$ & $\ddagger$ \\
\hline Youth Poll 3 (Nov 01) & $\ddagger$ & $\ddagger$ & $\ddagger$ & $\ddagger$ & $\ddagger$ & $\ddagger$ & $\ddagger$ & $\ddagger$ & $\ddagger$ \\
\hline Youth Poll 4 (Nov 02) & $\ddagger$ & $\ddagger$ & $\ddagger$ & $\ddagger$ & $\ddagger$ & $\ddagger$ & $\ddagger$ & $\ddagger$ & $\ddagger$ \\
\hline Youth Poll 5 (June 03) & 6.3 & 9.6 & 7.0 & 10.6 & 13.0 & 12.8 & 13.8 & 8.1 & 12.7 \\
\hline Youth Poll 6 (Nov 03) & 10.6 & 14.8 & 11.8 & 10.5 & 12.0 & 17.8 & 18.2 & 9.8 & 13.0 \\
\hline Youth Poll 7 (May 04) & 11.2 & 11.3 & 11.2 & 3.6 & 11.4 & 6.5 & 15.4 & 14.3 & 13.0 \\
\hline Youth Poll 8 (Nov 04) & 6.6 & 10.0 & 9.5 & 11.2 & 10.2 & 12.2 & 12.7 & 11.8 & 11.7 \\
\hline Male (age 16-21) & & & & & ercent $(9$ & & & & \\
\hline (definitely \& probably) & & & & Geo & aphic $\mathbf{R}$ & gion & & & \\
\hline Year & $\begin{array}{c}\text { New } \\
\text { England }\end{array}$ & $\begin{array}{c}\text { Mid } \\
\text { Atlantic }\end{array}$ & $\begin{array}{c}\text { East } \\
\text { North } \\
\text { Central } \\
\end{array}$ & $\begin{array}{c}\text { West } \\
\text { North } \\
\text { Central } \\
\end{array}$ & $\begin{array}{c}\text { South } \\
\text { Atlantic }\end{array}$ & $\begin{array}{c}\text { East } \\
\text { South } \\
\text { Central }\end{array}$ & $\begin{array}{c}\text { West } \\
\text { South } \\
\text { Central } \\
\end{array}$ & Mountain & Pacific \\
\hline Youth Poll 1 (Apr 01) & $\ddagger$ & $\ddagger$ & $\ddagger$ & $\neq$ & $\ddagger$ & $\ddagger$ & $\ddagger$ & $\ddagger$ & $\ddagger$ \\
\hline Youth Poll 2 (Aug 01) & $\ddagger$ & $\ddagger$ & $\ddagger$ & $\ddagger$ & $\ddagger$ & $\ddagger$ & $\ddagger$ & $\ddagger$ & $\ddagger$ \\
\hline Youth Poll 3 (Nov 01) & $\ddagger$ & $\ddagger$ & $\ddagger$ & $\ddagger$ & $\ddagger$ & $\ddagger$ & $\ddagger$ & $\ddagger$ & $\ddagger$ \\
\hline Youth Poll 4 (Nov 02) & $\ddagger$ & $\ddagger$ & $\ddagger$ & $\ddagger$ & $\ddagger$ & $\ddagger$ & $\ddagger$ & $\ddagger$ & $\ddagger$ \\
\hline Youth Poll 5 (June 03) & $\ddagger$ & 12.4 & 10.0 & 12.9 & 21.3 & $\ddagger$ & 16.4 & 8.2 & 16.6 \\
\hline Youth Poll 6 (Nov 03) & $\ddagger$ & 19.5 & 14.3 & 17.0 & 15.0 & $\ddagger$ & 25.9 & $\ddagger$ & 16.1 \\
\hline Youth Poll 7 (May 04) & $\ddagger$ & 13.2 & 16.4 & $\ddagger$ & 14.6 & $\ddagger$ & 19.5 & $\ddagger$ & 19.8 \\
\hline Youth Poll 8 (Nov 04) & $\ddagger$ & 17.5 & 14.3 & 14.2 & 14.0 & $\ddagger$ & 14.4 & 18.8 & 16.6 \\
\hline
\end{tabular}

Female (age 16-21)

(definitely \& probably)

Year

Youth Poll 1 (Apr 01)

Youth Poll 2 (Aug 01)

Youth Poll 3 (Nov 01)

Youth Poll 4 (Nov 02)

Youth Poll 5 (June 03)

Youth Poll 6 (Nov 03)

Youth Poll 7 (May 04)

Youth Poll 8 (Nov 04)
Percent (\%)

\section{Geographic Region}

West South East West

North Atlantic South South Mountain Pacific

$\begin{array}{ccccc}\text { Central } & \text { Central } & \text { Atlantic } & \text { Central } & \text { Central } \\ \ddagger & \ddagger & \ddagger & \ddagger & \ddagger\end{array}$

$\ddagger \quad \ddagger$

$\ddagger$

$\ddagger$

$\ddagger$

11.4

10.7

$\ddagger$

$\ddagger$

$\ddagger$

$+$

7.9

8.1

$\ddagger \quad 6.2$

4.8

8.5

4.4

$\ddagger$

9.0

9.2

11.7

6.6

9.9

$\ddagger \quad 9.0$

6.0

‡

8.2

$5.2 \quad 7.3$

11.3

4.9

6.3

‡

\footnotetext{
${ }^{44} \ddagger$ Reporting standard not met (too few cases).

Note: Changes in sample size and stratification were implemented following November 2002 Youth Poll.

Source: Department of Defense Polls, JAMRS, 2001-2004 (Question: FPP12).
} 


\section{Reserve Propensity}

JAMRS

TABLE 9-5. Youth Reserve propensity, by high school grades: $2001-2004^{45}$



Females (age 16-21)

(definitely \& probably)

Year

Youth Poll 1 (Apr 01)

Youth Poll 2 (Aug 01)

Youth Poll 3 (Nov 01)

Youth Poll 4 (Nov 02)

Youth Poll 5 (June 03)

Youth Poll 6 (Nov 03)

Youth Poll 7 (May 04)

Youth Poll 8 (Nov 04)

Percent (\%)

\section{High School Grades}

\begin{tabular}{ccccccc}
$\begin{array}{c}\text { Mostly } \\
\text { A's }\end{array}$ & $\begin{array}{c}\text { Mostly A's } \\
\text { \& B's }\end{array}$ & $\begin{array}{c}\text { Mostly } \\
\text { B's }\end{array}$ & $\begin{array}{c}\text { Mostly B's } \\
\text { \& C's }\end{array}$ & $\begin{array}{c}\text { Mostly } \\
\text { C's }\end{array}$ & $\begin{array}{c}\text { Mostly C's } \\
\text { \& D's }\end{array}$ & $\begin{array}{c}\text { Mostly D's } \\
\text { and Lower }\end{array}$ \\
\hline 7.3 & 6.0 & $\ddagger$ & 10.4 & $\ddagger$ & $\ddagger$ & $\ddagger$ \\
6.7 & 6.3 & 12.7 & 9.2 & $\ddagger$ & $\ddagger$ & $\ddagger$ \\
4.3 & 6.1 & 3.6 & 13.6 & $\ddagger$ & $\ddagger$ & $\ddagger$ \\
4.6 & 2.9 & 13.9 & 9.2 & $\ddagger$ & $\ddagger$ & $\ddagger$ \\
2.9 & 9.1 & 5.8 & 7.2 & $\ddagger$ & $\ddagger$ & $\ddagger$ \\
4.0 & 9.1 & 11.4 & 11.0 & $\ddagger$ & $\ddagger$ & $\ddagger$ \\
3.2 & 9.8 & 6.9 & 8.8 & $\ddagger$ & $\ddagger$ & $\ddagger$ \\
4.0 & 6.4 & 4.5 & 6.6 & $\ddagger$ & $\ddagger$ & $\ddagger$ \\
\hline
\end{tabular}

${ }^{45} \ddagger$ Reporting standard not met (too few cases).

Note: Changes in sample size and stratification were implemented following November 2002 Youth Poll.

Source: Department of Defense Polls, JAMRS, 2001-2004 (Question: FPP12).

Page A-45 DoD May 2004 Youth Poll 


\section{National Guard Propensity}

TABLE 10-1. Youth National Guard propensity: $2001-2004^{46}$

Male and Female (age 16-21)

Percent (\%)

\begin{tabular}{lcccc}
\cline { 2 - 4 } Year & Definitely & Probably & Probably Not & Definitely Not \\
\hline Youth Poll 1 (Apr 01) & .9 & 5.1 & 26.0 & 67.9 \\
Youth Poll 2 (Aug 01) & .8 & 5.4 & 28.7 & 65.2 \\
Youth Poll 3 (Nov 01) & .6 & 7.5 & 33.9 & 57.9 \\
Youth Poll 4 (Nov 02) & .3 & 6.7 & 30.8 & 62.0 \\
Youth Poll 5 (June 03) & .7 & 6.5 & 33.0 & 59.7 \\
Youth Poll 6 (Nov 03) & .6 & 7.9 & 31.6 & 59.7 \\
Youth Poll 7 (May 04) & .6 & 7.4 & 34.0 & 58.0 \\
Youth Poll 8 (Nov 04) & .4 & 6.8 & 32.3 & 60.4 \\
\hline
\end{tabular}

Male (age 16-21)

Percent (\%)

\begin{tabular}{lcccc}
\cline { 2 - 4 } Year & Definitely & Probably & Probably Not & Definitely Not \\
\hline Youth Poll 1 (Apr 01) & 1.2 & 7.3 & 33.9 & 57.6 \\
Youth Poll 2 (Aug 01) & 1.0 & 6.9 & 35.2 & 56.8 \\
Youth Poll 3 (Nov 01) & .4 & 9.5 & 43.2 & 46.7 \\
Youth Poll 4 (Nov 02) & .2 & 9.6 & 34.1 & 55.9 \\
Youth Poll 5 (June 03) & .8 & 9.0 & 38.8 & 51.2 \\
Youth Poll 6 (Nov 03) & .7 & 10.0 & 38.8 & 50.4 \\
Youth Poll 7 (May 04) & .8 & 9.2 & 41.1 & 48.8 \\
Youth Poll 8 (Nov 04) & .5 & 9.1 & 38.9 & 51.3 \\
\hline
\end{tabular}

Female (age 16-21)

Percent (\%)

Year

Definitely

Probably

Probably Not Definitely Not

Youth Poll 1 (Apr 01)

.6

3.0

18.8

77.4

Youth Poll 2 (Aug 01)

.5

3.9

22.5

73.1

Youth Poll 3 (Nov 01)

.7

5.7

25.2

68.4

Youth Poll 4 (Nov 02)

.4

3.9

27.7

67.8

Youth Poll 5 (June 03)

3.9

26.9

68.5

Youth Poll 6 (Nov 03)

.6

5.8

24.3

69.1

Youth Poll 7 (May 04)

5.5

26.6

67.4

Youth Poll 8 (Nov 04)

.4

4.4

25.6

69.7

\footnotetext{
${ }^{46} \ddagger$ Reporting standard not met (too few cases).

Note: Changes in sample size and stratification were implemented following November 2002 Youth Poll.

Source: Department of Defense Polls, JAMRS, 2001-2004 (Question: FPP11).
} 


\section{National Guard Propensity}

TABLE 10-2. Youth National Guard propensity, by race/ethnicity: $2001-2004^{47}$

\begin{tabular}{|c|c|c|c|c|}
\hline \multirow{3}{*}{$\begin{array}{l}\text { Male and Female (age 16-21) } \\
\text { (definitely \& probably) } \\
\text { Year }\end{array}$} & \multicolumn{4}{|c|}{ Percent (\%) } \\
\hline & \multicolumn{4}{|c|}{ Race/ethnicity } \\
\hline & Total & White & Black & Hispanic \\
\hline Youth Poll 1 (Apr 01) & 5.9 & 4.6 & 7.7 & 9.8 \\
\hline Youth Poll 2 (Aug 01) & 6.1 & 2.9 & 12.4 & 12.0 \\
\hline Youth Poll 3 (Nov 01) & 8.1 & 5.9 & 11.8 & 13.2 \\
\hline Youth Poll 4 (Nov 02) & 7.0 & 4.5 & 10.9 & 11.8 \\
\hline Youth Poll 5 (June 03) & 7.2 & 5.0 & 10.4 & 12.6 \\
\hline Youth Poll 6 (Nov 03) & 8.6 & 5.9 & 11.9 & 14.3 \\
\hline Youth Poll 7 (May 04) & 8.0 & 5.2 & 12.8 & 14.0 \\
\hline Youth Poll 8 (Nov 04) & 7.2 & 5.2 & 8.3 & 13.0 \\
\hline Male (age 16-21) & \multicolumn{4}{|c|}{ Percent (\%) } \\
\hline (definitely \& probably) & \multicolumn{4}{|c|}{ Race/ethnicity } \\
\hline Year & Total & White & Black & Hispanic \\
\hline Youth Poll 1 (Apr 01) & 8.4 & 7.7 & 12.6 & 8.7 \\
\hline Youth Poll 2 (Aug 01) & 8.0 & 4.3 & 20.9 & 12.6 \\
\hline Youth Poll 3 (Nov 01) & 9.8 & 8.7 & 8.6 & 14.4 \\
\hline Youth Poll 4 (Nov 02) & 9.9 & 6.6 & 16.3 & 16.5 \\
\hline Youth Poll 5 (June 03) & 9.7 & 7.0 & 13.2 & 16.6 \\
\hline Youth Poll 6 (Nov 03) & 10.7 & 8.1 & 15.8 & 17.2 \\
\hline Youth Poll 7 (May 04) & 10.0 & 7.1 & 13.6 & 17.2 \\
\hline Youth Poll 8 (Nov 04) & 9.6 & 8.2 & 9.4 & 13.9 \\
\hline Female (age 16-21) & \multicolumn{4}{|c|}{ Percent (\%) } \\
\hline (definitely \& probably) & \multicolumn{4}{|c|}{ Race/ethnicity } \\
\hline Year & Total & White & Black & Hispanic \\
\hline Youth Poll 1 (Apr 01) & 3.6 & 1.5 & 4.4 & 10.7 \\
\hline Youth Poll 2 (Aug 01) & 4.4 & 1.5 & 7.0 & 11.4 \\
\hline Youth Poll 3 (Nov 01) & 6.5 & 3.0 & 13.6 & 12.1 \\
\hline Youth Poll 4 (Nov 02) & 4.3 & 2.4 & 5.7 & 8.1 \\
\hline Youth Poll 5 (June 03) & 4.5 & 2.8 & 8.1 & 8.5 \\
\hline Youth Poll 6 (Nov 03) & 6.4 & 3.6 & 8.7 & 11.5 \\
\hline Youth Poll 7 (May 04) & 5.9 & 3.2 & 12.1 & 10.7 \\
\hline Youth Poll 8 (Nov 04) & 4.7 & 1.8 & 7.6 & 12.0 \\
\hline
\end{tabular}

${ }^{47}$ Due to relatively small sample sizes, American Indian, Alaska Native, Asian, and Pacific Islander are included in the total but are not shown separately. Black includes African American, and Hispanic includes Latino. Race categories exclude Hispanic origin unless specified otherwise.

‡Reporting standard not met (too few cases).

Note: Changes in sample size and stratification were implemented following November 2002 Youth Poll.

Source: Department of Defense Polls, JAMRS, 2001-2004 (Question: FPP11). 


\section{National Guard Propensity}

JAMRS

TABLE 10-3. Youth National Guard propensity, by age: $2001-2004^{48}$

\begin{tabular}{lcccccc} 
Male and Female (age 16-21) & \multicolumn{7}{c}{ Percent (\%) } \\
\cline { 2 - 7 } $\begin{array}{l}\text { (definitely \& probably) } \\
\text { Year }\end{array}$ & $\mathbf{1 6}$ & $\mathbf{1 7}$ & $\mathbf{1 8}$ & $\mathbf{1 9}$ & $\mathbf{2 0}$ & $\mathbf{2 1}$ \\
\hline & 9.4 & 6.6 & 5.7 & 5.0 & 3.1 & 5.9 \\
\hline Youth Poll 1 (Apr 01) & 8.6 & 6.0 & 6.8 & 6.0 & 5.4 & 3.6 \\
Youth Poll 2 (Aug 01) & 9.4 & 10.8 & 8.2 & 7.6 & 7.1 & 5.3 \\
Youth Poll 3 (Nov 01) & 10.5 & 9.7 & 7.2 & 3.2 & 7.6 & 4.0 \\
Youth Poll 4 (Nov 02) & 9.7 & 9.0 & 5.8 & 7.5 & 4.0 & 7.0 \\
Youth Poll 5 (June 03) & 11.0 & 10.0 & 9.4 & 6.5 & 5.6 & 8.4 \\
Youth Poll 6 (Nov 03) & 10.1 & 9.3 & 7.7 & 7.0 & 4.1 & 9.1 \\
Youth Poll 7 (May 04) & 10.0 & 8.1 & 5.9 & 4.6 & 8.2 & 5.9 \\
Youth Poll 8 (Nov 04) & & & & & & \\
\hline
\end{tabular}

Male (age 16-21)

(definitely \& probably)

\section{Year}

Youth Poll 1 (Apr 01)

Youth Poll 2 (Aug 01)

Youth Poll 3 (Nov 01)

Youth Poll 4 (Nov 02)

Youth Poll 5 (June 03)

Youth Poll 6 (Nov 03)

Youth Poll 7 (May 04)

Youth Poll 8 (Nov 04)

\begin{tabular}{cccccc}
\multicolumn{7}{c}{ Percent (\%) } \\
\hline \multicolumn{7}{c}{ Age } \\
\hline 16 & $\mathbf{1 7}$ & $\mathbf{1 8}$ & $\mathbf{1 9}$ & $\mathbf{2 0}$ & $\mathbf{2 1}$ \\
\hline 12.3 & 8.5 & 7.6 & 9.9 & 2.3 & 9.7 \\
12.8 & 7.5 & 8.7 & 7.2 & 7.8 & 2.1 \\
11.3 & 12.3 & 9.6 & 8.3 & 9.2 & 8.0 \\
13.6 & 12.4 & 12.8 & 2.8 & 10.8 & 6.0 \\
10.6 & 11.5 & 8.5 & 10.6 & 5.4 & 12.2 \\
13.3 & 12.7 & 12.0 & 7.1 & 6.7 & 11.5 \\
11.6 & 11.2 & 8.9 & 8.9 & 6.1 & 13.2 \\
12.1 & 10.4 & 9.9 & 6.3 & 12.9 & 5.7 \\
\hline
\end{tabular}

\section{Female (age 16-21)}

(definitely \& probably)

Year

Youth Poll 1 (Apr 01)

Youth Poll 2 (Aug 01)

Youth Poll 3 (Nov 01)

Youth Poll 4 (Nov 02)

Youth Poll 5 (June 03)

Youth Poll 6 (Nov 03)

Youth Poll 7 (May 04)

Youth Poll 8 (Nov 04)

\begin{tabular}{cccccc}
\multicolumn{7}{c}{ Percent (\%) } \\
\hline $\mathbf{7}$ & Age \\
\hline 6.6 & $\mathbf{1 7}$ & $\mathbf{1 8}$ & $\mathbf{1 9}$ & $\mathbf{2 0}$ & $\mathbf{2 1}$ \\
3.4 & 4.2 & 3.9 & 0.0 & 3.8 & 3.2 \\
7.6 & 4.4 & 5.1 & 5.0 & 3.4 & 4.7 \\
7.1 & 9.1 & 6.9 & 7.1 & 5.4 & 2.7 \\
8.9 & 7.1 & 1.4 & 3.5 & 4.1 & 2.4 \\
8.7 & 6.4 & 2.9 & 4.3 & 2.5 & 2.1 \\
8.6 & 6.9 & 6.8 & 5.8 & 4.6 & 5.4 \\
7.7 & 7.6 & 6.4 & 5.1 & 1.9 & 4.9 \\
& 5.7 & 1.8 & 2.9 & 3.5 & 6.1 \\
\hline
\end{tabular}

${ }^{48}$ ‡Reporting standard not met (too few cases).

Note: Changes in sample size and stratification were implemented following November 2002 Youth Poll.

Source: Department of Defense Polls, JAMRS, 2001-2004 (Question: FPP11). 


\section{National Guard Propensity}

JAMRS

TABLE 10-4. Youth National Guard propensity, by geographic region: $2001-2004^{49}$

\begin{tabular}{|c|c|c|c|c|c|c|c|c|c|}
\hline Male and Female (age & $6-21)$ & & & & rcent $(\%$ & & & & \\
\hline (definitely \& probably) & & & & Geo & raphic $\mathrm{F}$ & gion & & & \\
\hline Year & $\begin{array}{l}\text { New } \\
\text { England }\end{array}$ & $\begin{array}{c}\text { Mid } \\
\text { Atlantic }\end{array}$ & $\begin{array}{c}\text { East } \\
\text { North } \\
\text { Central }\end{array}$ & $\begin{array}{c}\text { West } \\
\text { North } \\
\text { Central }\end{array}$ & $\begin{array}{c}\text { South } \\
\text { Atlantic }\end{array}$ & $\begin{array}{l}\text { East } \\
\text { South } \\
\text { Central }\end{array}$ & $\begin{array}{c}\text { West } \\
\text { South } \\
\text { Central }\end{array}$ & Mountain & Pacific \\
\hline Youth Poll 1 (Apr 01) & $\ddagger$ & $\ddagger$ & $\ddagger$ & $\ddagger$ & $\ddagger$ & $\ddagger$ & $\ddagger$ & $\ddagger$ & $\ddagger$ \\
\hline Youth Poll 2 (Aug 01) & $\ddagger$ & $\ddagger$ & $\ddagger$ & $\ddagger$ & $\ddagger$ & $\ddagger$ & $\ddagger$ & $\ddagger$ & $\ddagger$ \\
\hline Youth Poll 3 (Nov 01) & $\ddagger$ & $\ddagger$ & $\ddagger$ & $\ddagger$ & $\ddagger$ & $\ddagger$ & $\ddagger$ & $\ddagger$ & $\ddagger$ \\
\hline Youth Poll 4 (Nov 02) & $\ddagger$ & $\ddagger$ & $\ddagger$ & $\ddagger$ & $\ddagger$ & $\ddagger$ & $\ddagger$ & $\ddagger$ & $\ddagger$ \\
\hline Youth Poll 5 (June 03) & 7.3 & 6.7 & 4.9 & 5.3 & 9.0 & 11.0 & 8.4 & 5.9 & 7.4 \\
\hline Youth Poll 6 (Nov 03) & 2.6 & 9.5 & 6.8 & 13.4 & 8.7 & 9.7 & 9.6 & 5.8 & 8.6 \\
\hline Youth Poll 7 (May 04) & 5.0 & 8.0 & 9.9 & 4.1 & 6.4 & 5.4 & 9.7 & 13.2 & 7.6 \\
\hline Youth Poll 8 (Nov 04) & 5.6 & 6.4 & 6.0 & 6.8 & 7.6 & 8.6 & 10.4 & 6.5 & 6.6 \\
\hline Male (age 16-21) & & & & & ercent $(9$ & & & & \\
\hline (definitely \& probably) & & & & Geo & aphic $\mathbf{R}$ & gion & & & \\
\hline Year & $\begin{array}{c}\text { New } \\
\text { England }\end{array}$ & $\begin{array}{c}\text { Mid } \\
\text { Atlantic }\end{array}$ & $\begin{array}{c}\text { East } \\
\text { North } \\
\text { Central } \\
\end{array}$ & $\begin{array}{c}\text { West } \\
\text { North } \\
\text { Central } \\
\end{array}$ & $\begin{array}{c}\text { South } \\
\text { Atlantic }\end{array}$ & $\begin{array}{c}\text { East } \\
\text { South } \\
\text { Central } \\
\end{array}$ & $\begin{array}{c}\text { West } \\
\text { South } \\
\text { Central } \\
\end{array}$ & Mountain & Pacific \\
\hline Youth Poll 1 (Apr 01) & $\ddagger$ & $\ddagger$ & $\ddagger$ & $\neq$ & $\ddagger$ & $\ddagger$ & $\ddagger$ & $\ddagger$ & $\ddagger$ \\
\hline Youth Poll 2 (Aug 01) & $\ddagger$ & $\ddagger$ & $\ddagger$ & $\ddagger$ & $\ddagger$ & $\ddagger$ & $\ddagger$ & $\ddagger$ & $\ddagger$ \\
\hline Youth Poll 3 (Nov 01) & $\ddagger$ & $\ddagger$ & $\ddagger$ & $\ddagger$ & $\ddagger$ & $\ddagger$ & $\ddagger$ & $\ddagger$ & $\ddagger$ \\
\hline Youth Poll 4 (Nov 02) & $\ddagger$ & $\ddagger$ & $\ddagger$ & $\ddagger$ & $\ddagger$ & $\ddagger$ & $\ddagger$ & $\ddagger$ & $\ddagger$ \\
\hline Youth Poll 5 (June 03) & $\ddagger$ & 8.5 & 6.7 & 7.0 & 12.9 & $\ddagger$ & 11.8 & 7.2 & 10.3 \\
\hline Youth Poll 6 (Nov 03) & $\ddagger$ & 11.5 & 7.0 & 22.3 & 11.7 & $\ddagger$ & 11.1 & $\ddagger$ & 9.7 \\
\hline Youth Poll 7 (May 04) & $\ddagger$ & 11.2 & 12.8 & $\ddagger$ & 7.8 & $\ddagger$ & 14.5 & $\ddagger$ & 11.5 \\
\hline Youth Poll 8 (Nov 04) & $\ddagger$ & 10.7 & 7.3 & 10.4 & 10.7 & $\ddagger$ & 11.9 & 8.2 & 8.9 \\
\hline Female (age 16-21) & & & & & ercent $(0$ & & & & \\
\hline (definitely \& probably) & & & & Geo & aphic $\mathrm{R}$ & gion & & & \\
\hline Year & $\begin{array}{c}\text { New } \\
\text { England }\end{array}$ & $\begin{array}{c}\text { Mid } \\
\text { Atlantic }\end{array}$ & $\begin{array}{c}\text { East } \\
\text { North } \\
\text { Central } \\
\end{array}$ & $\begin{array}{c}\text { West } \\
\text { North } \\
\text { Central } \\
\end{array}$ & $\begin{array}{c}\text { South } \\
\text { Atlantic }\end{array}$ & $\begin{array}{c}\text { East } \\
\text { South } \\
\text { Central } \\
\end{array}$ & $\begin{array}{c}\text { West } \\
\text { South } \\
\text { Central } \\
\end{array}$ & Mountain & Pacific \\
\hline Youth Poll 1 (Apr 01) & $\ddagger$ & $\ddagger$ & $\ddagger$ & $\ddagger$ & $\mp$ & $\ddagger$ & $\ddagger$ & $\ddagger$ & $\ddagger$ \\
\hline Youth Poll 2 (Aug 01) & $\ddagger$ & $\ddagger$ & $\ddagger$ & $\ddagger$ & $\ddagger$ & $\ddagger$ & $\ddagger$ & $\ddagger$ & $\ddagger$ \\
\hline Youth Poll 3 (Nov 01) & $\ddagger$ & $\ddagger$ & $\ddagger$ & $\ddagger$ & $\ddagger$ & $\ddagger$ & $\ddagger$ & $\ddagger$ & $\ddagger$ \\
\hline Youth Poll 4 (Nov 02) & $\ddagger$ & $\ddagger$ & $\ddagger$ & $\ddagger$ & $\ddagger$ & $\ddagger$ & $\ddagger$ & $\ddagger$ & $\ddagger$ \\
\hline Youth Poll 5 (June 03) & $\ddagger$ & 4.5 & 3.5 & 3.8 & 4.9 & $\ddagger$ & 5.5 & 4.3 & 4.1 \\
\hline Youth Poll 6 (Nov 03) & $\ddagger$ & 7.6 & 6.6 & 4.3 & 5.8 & 7.4 & 8.2 & 3.7 & 7.5 \\
\hline Youth Poll 7 (May 04) & $\ddagger$ & 4.3 & 7.0 & $\ddagger$ & 5.2 & $\ddagger$ & 5.5 & $\ddagger$ & 3.8 \\
\hline Youth Poll 8 (Nov 04) & $\ddagger$ & 2.0 & 4.8 & 2.1 & 4.7 & $\ddagger$ & 9.1 & 4.7 & 3.9 \\
\hline
\end{tabular}

${ }^{49}$ ‡Reporting standard not met (too few cases).

Note: Changes in sample size and stratification were implemented following November 2002 Youth Poll.

Source: Department of Defense Polls, JAMRS, 2001-2004 (Question: FPP11). 


\section{National Guard Propensity}

JAMRS

TABLE 10-5. Youth National Guard propensity, by high school grades: $2001-2004^{50}$

\begin{tabular}{|c|c|c|c|c|c|c|c|}
\hline \multicolumn{4}{|c|}{ Male and Female (age 16-21) } & \multicolumn{4}{|c|}{ Percent (\%) } \\
\hline \multirow{2}{*}{$\begin{array}{l}\text { (definitely \& probably) } \\
\text { Year }\end{array}$} & \multicolumn{7}{|c|}{ High School Grades } \\
\hline & $\begin{array}{l}\text { Mostly } \\
\text { A's }\end{array}$ & $\begin{array}{l}\text { Mostly A's } \\
\text { \& B's }\end{array}$ & $\begin{array}{l}\text { Mostly } \\
\text { B's }\end{array}$ & $\begin{array}{c}\text { Mostly B's } \\
\text { \& C's }\end{array}$ & $\begin{array}{l}\text { Mostly } \\
\text { C's }\end{array}$ & $\begin{array}{l}\text { Mostly C's } \\
\text { \& D's }\end{array}$ & $\begin{array}{l}\text { Mostly D's } \\
\text { and Lower }\end{array}$ \\
\hline Youth Poll 1 (Apr 01) & 1.1 & 4.3 & 8.3 & 8.3 & $\ddagger$ & 8.0 & $\ddagger$ \\
\hline Youth Poll 2 (Aug 01) & 3.6 & 5.0 & 6.7 & 7.7 & $\ddagger$ & 7.4 & $\ddagger$ \\
\hline Youth Poll 3 (Nov 01) & 4.7 & 6.5 & 7.3 & 11.6 & $\ddagger$ & $\ddagger$ & $\ddagger$ \\
\hline Youth Poll 4 (Nov 02) & 1.7 & 3.2 & 8.8 & 11.0 & 13.4 & $\ddagger$ & $\ddagger$ \\
\hline Youth Poll 5 (June 03) & 2.1 & 6.9 & 5.3 & 11.3 & 7.8 & 8.3 & $\ddagger$ \\
\hline Youth Poll 6 (Nov 03) & 5.9 & 8.8 & 6.2 & 10.7 & 6.2 & 12.6 & $\ddagger$ \\
\hline Youth Poll 7 (May 04) & 3.3 & 8.5 & 8.0 & 8.6 & 9.0 & 16.1 & $\ddagger$ \\
\hline Youth Poll 8 (Nov 04) & 3.6 & 7.1 & 6.1 & 9.0 & 6.9 & 7.7 & $\ddagger$ \\
\hline Male (age 16-21) & \multicolumn{7}{|c|}{ Percent (\%) } \\
\hline (definitely \& probably) & \multicolumn{7}{|c|}{ High School Grades } \\
\hline Year & $\begin{array}{c}\text { Mostly } \\
\text { A's }\end{array}$ & $\begin{array}{l}\text { Mostly A's } \\
\text { \& B's }\end{array}$ & $\begin{array}{l}\text { Mostly } \\
\text { B's }\end{array}$ & $\begin{array}{l}\text { Mostly B's } \\
\text { \& C's }\end{array}$ & $\begin{array}{l}\text { Mostly } \\
\text { C's }\end{array}$ & $\begin{array}{l}\text { Mostly C's } \\
\text { \& D's }\end{array}$ & $\begin{array}{l}\text { Mostly D's } \\
\text { and Lower }\end{array}$ \\
\hline Youth Poll 1 (Apr 01) & $\ddagger$ & 5.7 & $\ddagger$ & 10.9 & $\ddagger$ & $\ddagger$ & $\ddagger$ \\
\hline Youth Poll 2 (Aug 01) & $\ddagger$ & 6.9 & 8.6 & 10.5 & $\ddagger$ & $\ddagger$ & $\ddagger$ \\
\hline Youth Poll 3 (Nov 01) & 4.6 & 6.7 & 9.7 & 13.9 & $\ddagger$ & $\ddagger$ & $\ddagger$ \\
\hline Youth Poll 4 (Nov 02) & $\ddagger$ & 5.0 & 6.6 & 13.9 & $\ddagger$ & $\ddagger$ & $\ddagger$ \\
\hline Youth Poll 5 (June 03) & 2.4 & 9.3 & 8.6 & 14.3 & 6.8 & 9.4 & $\ddagger$ \\
\hline Youth Poll 6 (Nov 03) & 9.4 & 11.1 & 7.0 & 12.5 & 6.6 & 14.6 & $\ddagger$ \\
\hline Youth Poll 7 (May 04) & 4.4 & 11.5 & 8.8 & 9.8 & $\ddagger$ & 19.4 & $\ddagger$ \\
\hline Youth Poll 8 (Nov 04) & 5.7 & 10.6 & 5.7 & 12.1 & 7.4 & $\ddagger$ & $\ddagger$ \\
\hline
\end{tabular}

Females (age 16-21)

(definitely \& probably)

\section{Year}

Youth Poll 1 (Apr 01)

Youth Poll 2 (Aug 01)

Youth Poll 3 (Nov 01)

Youth Poll 4 (Nov 02)

Youth Poll 5 (June 03)

Youth Poll 6 (Nov 03)

Youth Poll 7 (May 04)

Youth Poll 8 (Nov 04)

5.7

\section{Percent (\%)}

Percent (\%)

\section{High School Grades}

\begin{tabular}{ccccccc}
\hline $\begin{array}{c}\text { Mostly } \\
\text { A's }\end{array}$ & $\begin{array}{c}\text { Mostly A's } \\
\text { \& B's }\end{array}$ & $\begin{array}{c}\text { Mostly } \\
\text { B's }\end{array}$ & $\begin{array}{c}\text { Mostly B's } \\
\text { \& C's }\end{array}$ & $\begin{array}{c}\text { Mostly } \\
\text { C's }\end{array}$ & $\begin{array}{c}\text { Mostly C's } \\
\text { \& D's }\end{array}$ & $\begin{array}{c}\text { Mostly D's } \\
\text { and Lower }\end{array}$ \\
\hline .4 & 3.3 & $\ddagger$ & 5.2 & $\ddagger$ & $\ddagger$ & $\ddagger$ \\
3.6 & 3.5 & 5.1 & 4.6 & $\ddagger$ & $\ddagger$ & $\ddagger$ \\
4.8 & 6.3 & 4.9 & 9.0 & $\ddagger$ & $\ddagger$ & $\ddagger$ \\
1.3 & 2.0 & 10.8 & 6.8 & $\ddagger$ & $\ddagger$ & $\ddagger$ \\
1.8 & 5.1 & 2.2 & 6.7 & $\ddagger$ & $\ddagger$ & $\ddagger$ \\
3.3 & 7.1 & 5.4 & 8.6 & $\ddagger$ & $\ddagger$ & $\ddagger$ \\
2.5 & 6.4 & 7.1 & 7.0 & $\ddagger$ & $\ddagger$ & $\ddagger$ \\
2.3 & 4.1 & 6.5 & 4.9 & $\ddagger$ & $\ddagger$ & $\ddagger$ \\
\hline
\end{tabular}

${ }^{50} \ddagger$ Reporting standard not met (too few cases).

Note: Changes in sample size and stratification were implemented following November 2002 Youth Poll.

Source: Department of Defense Polls, JAMRS, 2001-2004 (Question: FPP11). 


\section{Military Consideration}

TABLE 11-1. Youth consideration of military service: $2001-2004^{51}$

Male and Female (age 16-21)

\begin{tabular}{lccc}
\cline { 2 - 4 } Year & Never & Some & Serious \\
\hline Youth Poll 1 (Apr 01) & 34.7 & 47.4 & 17.9 \\
Youth Poll 2 (Aug 01) & 30.4 & 51.7 & 17.9 \\
Youth Poll 3 (Nov 01) & 28.9 & 50.4 & 20.7 \\
Youth Poll 4 (Nov 02) & 29.2 & 49.8 & 20.8 \\
Youth Poll 5 (June 03) & 29.1 & 51.3 & 19.6 \\
Youth Poll 6 (Nov 03) & 27.7 & 50.5 & 21.8 \\
Youth Poll 7 (May 04) & 28.6 & 49.1 & 22.3 \\
Youth Poll 8 (Nov 04) & 33.0 & 46.8 & 20.2 \\
\hline
\end{tabular}

Male (age 16-21)

\begin{tabular}{lccc}
\cline { 2 - 3 } Year & Never & Some & Serious \\
\hline Youth Poll 1 (Apr 01) & 22.5 & 54.1 & 23.4 \\
Youth Poll 2 (Aug 01) & 23.3 & 53.5 & 23.1 \\
Youth Poll 3 (Nov 01) & 19.3 & 50.9 & 29.8 \\
Youth Poll 4 (Nov 02) & 21.2 & 50.4 & 28.2 \\
Youth Poll 5 (June 03) & 19.9 & 54.0 & 26.1 \\
Youth Poll 6 (Nov 03) & 19.5 & 51.5 & 28.9 \\
Youth Poll 7 (May 04) & 21.5 & 49.6 & 28.9 \\
Youth Poll 8 (Nov 04) & 23.5 & 47.8 & 28.6 \\
\hline
\end{tabular}

Female (age 16-21)

\begin{tabular}{lccc}
\cline { 2 - 4 } Year & Never & Some & Serious \\
\hline Youth Poll 1 (Apr 01) & 45.9 & 41.3 & 12.9 \\
Youth Poll 2 (Aug 01) & 37.0 & 50.0 & 12.9 \\
Youth Poll 3 (Nov 01) & 37.8 & 49.9 & 12.3 \\
Youth Poll 4 (Nov 02) & 36.9 & 49.3 & 13.8 \\
Youth Poll 5 (June 03) & 38.5 & 48.5 & 12.9 \\
Youth Poll 6 (Nov 03) & 35.9 & 49.5 & 14.6 \\
Youth Poll 7 (May 04) & 35.9 & 48.5 & 15.6 \\
Youth Poll 8 (Nov 04) & 42.7 & 45.7 & 11.6 \\
\hline
\end{tabular}

${ }^{51}$ ‡Reporting standard not met (too few cases).

Note: Changes in sample size and stratification were implemented following November 2002 Youth Poll.

Source: Department of Defense Polls, JAMRS, 2001-2004 (Question: FPP15). 


\section{Military Consideration}

TABLE 11-2. Youth consideration of military service, by race/ethnicity: $2001-2004^{52}$

\begin{tabular}{|c|c|c|c|}
\hline \multirow{3}{*}{$\begin{array}{l}\text { Male and Female (age 16-21) } \\
\text { (never considered) } \\
\text { Year }\end{array}$} & \multicolumn{3}{|c|}{ Percent (\%) } \\
\hline & \multicolumn{3}{|c|}{ Race/ethnicity } \\
\hline & White & Black & Hispanic \\
\hline Youth Poll 1 (Apr 01) & 33.3 & 39.3 & 34.6 \\
\hline Youth Poll 2 (Aug 01) & 30.8 & 35.0 & 25.9 \\
\hline Youth Poll 3 (Nov 01) & 27.3 & 34.5 & 29.3 \\
\hline Youth Poll 4 (Nov 02) & 27.6 & 38.0 & 29.8 \\
\hline Youth Poll 5 (June 03) & 27.7 & 38.5 & 24.2 \\
\hline Youth Poll 6 (Nov 03) & 26.6 & 33.7 & 27.4 \\
\hline Youth Poll 7 (May 04) & 26.9 & 38.2 & 26.3 \\
\hline Youth Poll 8 (Nov 04) & 30.3 & 41.2 & 36.4 \\
\hline Male (age 16-21) & \multicolumn{3}{|c|}{ Percent (\%) } \\
\hline (never considered) & \multicolumn{3}{|c|}{ Race/ethnicity } \\
\hline Year & White & Black & Hispanic \\
\hline Youth Poll 1 (Apr 01) & 21.6 & 28.3 & 20.6 \\
\hline Youth Poll 2 (Aug 01) & 22.4 & 33.6 & 21.2 \\
\hline Youth Poll 3 (Nov 01) & 16.5 & 30.8 & 22.9 \\
\hline Youth Poll 4 (Nov 02) & 19.6 & 33.7 & 17.7 \\
\hline Youth Poll 5 (June 03) & 17.0 & 34.3 & 17.9 \\
\hline Youth Poll 6 (Nov 03) & 17.2 & 28.9 & 20.7 \\
\hline Youth Poll 7 (May 04) & 17.7 & 37.1 & 24.2 \\
\hline Youth Poll 8 (Nov 04) & 19.4 & 39.5 & 27.7 \\
\hline Female (age 16-21) & \multicolumn{3}{|c|}{ Percent (\%) } \\
\hline (never considered) & \multicolumn{3}{|c|}{ Race/ethnicity } \\
\hline Year & White & Black & Hispanic \\
\hline Youth Poll 1 (Apr 01) & 44.9 & 46.8 & 46.7 \\
\hline Youth Poll 2 (Aug 01) & 39.4 & 35.9 & 30.8 \\
\hline Youth Poll 3 (Nov 01) & 38.2 & 36.6 & 36.2 \\
\hline Youth Poll 4 (Nov 02) & 35.6 & 42.3 & 39.1 \\
\hline Youth Poll 5 (June 03) & 39.1 & 41.9 & 30.8 \\
\hline Youth Poll 6 (Nov 03) & 36.5 & 37.7 & 34.0 \\
\hline Youth Poll 7 (May 04) & 37.1 & 39.1 & 28.4 \\
\hline Youth Poll 8 (Nov 04) & 42.5 & 42.3 & 45.5 \\
\hline
\end{tabular}

\footnotetext{
${ }^{52}$ Due to relatively small sample sizes, American Indian, Alaska Native, Asian, and Pacific Islander are included in the total but are not shown separately. Black includes African American, and Hispanic includes Latino. Race categories exclude Hispanic origin unless specified otherwise.

‡Reporting standard not met (too few cases).

Note: Changes in sample size and stratification were implemented following November 2002 Youth Poll.

Source: Department of Defense Polls, JAMRS, 2001-2004 (Question: FPP15).
}

Page A-52 DoD May 2004 Youth Poll 


\section{Military Consideration}

JAMRS

TABLE 11-3. Youth consideration of military service, by age: $2001-2004^{53}$

\begin{tabular}{lcccccc} 
Male and Female (age 16-21) & \multicolumn{7}{c}{ Percent (\%) } \\
\cline { 2 - 7 } (never considered) & \multicolumn{7}{c}{ Age } \\
\cline { 2 - 7 } Year & 31.6 & $\mathbf{1 6}$ & $\mathbf{1 7}$ & $\mathbf{1 9}$ & $\mathbf{2 0}$ & $\mathbf{2 1}$ \\
\hline Youth Poll 1 (Apr 01) & 32.9 & 32.8 & 26.6 & 34.4 & 25.1 & 30.1 \\
Youth Poll 2 (Aug 01) & 31.1 & 28.4 & 27.0 & 32.2 & 25.5 & 28.8 \\
Youth Poll 3 (Nov 01) & 34.6 & 31.6 & 29.9 & 28.2 & 21.7 & 29.6 \\
Youth Poll 4 (Nov 02) & 28.1 & 28.7 & 24.8 & 31.4 & 31.7 & 30.2 \\
Youth Poll 5 (June 03) & 30.1 & 28.8 & 25.7 & 25.9 & 28.4 & 27.1 \\
Youth Poll 6 (Nov 03) & 27.5 & 26.4 & 30.9 & 25.6 & 33.1 & 28.2 \\
Youth Poll 7 (May 04) & 34.5 & 30.8 & 34.9 & 32.9 & 29.9 & 34.8 \\
Youth Poll 8 (Nov 04) & & & & & 37.2 & \\
\hline
\end{tabular}

\begin{tabular}{lcccccc} 
Male (age 16-21) & \multicolumn{7}{c}{ Percent (\%) } \\
\cline { 2 - 7 } (never considered) & \multicolumn{7}{c}{ Age } \\
\cline { 2 - 7 } Year & $\mathbf{1 6}$ & $\mathbf{1 7}$ & $\mathbf{1 8}$ & $\mathbf{1 9}$ & $\mathbf{2 0}$ & $\mathbf{2 1}$ \\
\hline Youth Poll 1 (Apr 01) & 25.3 & 21.9 & 16.1 & 24.8 & 27.8 & 18.5 \\
Youth Poll 2 (Aug 01) & 24.6 & 21.6 & 21.3 & 28.1 & 22.7 & 21.3 \\
Youth Poll 3 (Nov 01) & 21.4 & 19.2 & 22.4 & 24.1 & 12.6 & 15.4 \\
Youth Poll 4 (Nov 02) & 26.4 & 21.6 & 22.3 & 23.2 & 12.8 & 21.7 \\
Youth Poll 5 (June 03) & 19.5 & 23.8 & 16.5 & 21.2 & 21.3 & 17.1 \\
Youth Poll 6 (Nov 03) & 22.3 & 24.7 & 18.0 & 16.0 & 22.2 & 12.3 \\
Youth Poll 7 (May 04) & 21.2 & 18.4 & 24.0 & 17.4 & 25.6 & 21.9 \\
Youth Poll 8 (Nov 04) & 28.3 & 24.4 & 23.4 & 20.5 & 19.4 & 24.0 \\
\hline
\end{tabular}

\section{Female (age 16-21)}

(never considered)

Year

Youth Poll 1 (Apr 01)

Youth Poll 2 (Aug 01)

Youth Poll 3 (Nov 01)

Youth Poll 4 (Nov 02)

Youth Poll 5 (June 03)

Youth Poll 6 (Nov 03)

Youth Poll 7 (May 04)

Youth Poll 8 (Nov 04)

\begin{tabular}{cccccc}
\multicolumn{7}{c}{ Percent (\%) } \\
\hline $\mathbf{7}$ & Age \\
\hline $\mathbf{1 6}$ & $\mathbf{1 7}$ & $\mathbf{1 8}$ & $\mathbf{1 9}$ & $\mathbf{2 0}$ & $\mathbf{2 1}$ \\
\hline 37.6 & 46.2 & 47.3 & 50.1 & 49.2 & 44.7 \\
43.3 & 44.5 & 31.4 & 40.1 & 27.1 & 36.9 \\
40.4 & 38.4 & 31.7 & 38.7 & 36.0 & 41.7 \\
43.4 & 41.0 & 37.9 & 32.6 & 31.3 & 36.0 \\
37.0 & 33.9 & 33.4 & 41.6 & 43.1 & 42.6 \\
37.8 & 33.3 & 33.2 & 35.9 & 34.1 & 41.8 \\
33.9 & 34.1 & 38.6 & 34.0 & 40.9 & 34.5 \\
41.1 & 37.4 & 46.7 & 45.4 & 40.5 & 45.6 \\
\hline
\end{tabular}

${ }^{53}$ ₹Reporting standard not met (too few cases).

Note: Changes in sample size and stratification were implemented following November 2002 Youth Poll.

Source: Department of Defense Polls, JAMRS, 2001-2004 (Question: FPP15).

Page A-53 DoD May 2004 Youth Poll 


\section{Military Consideration}

JAMRS

TABLE 11-4. Youth consideration of military service, by geographic region: $2001-2004^{54}$

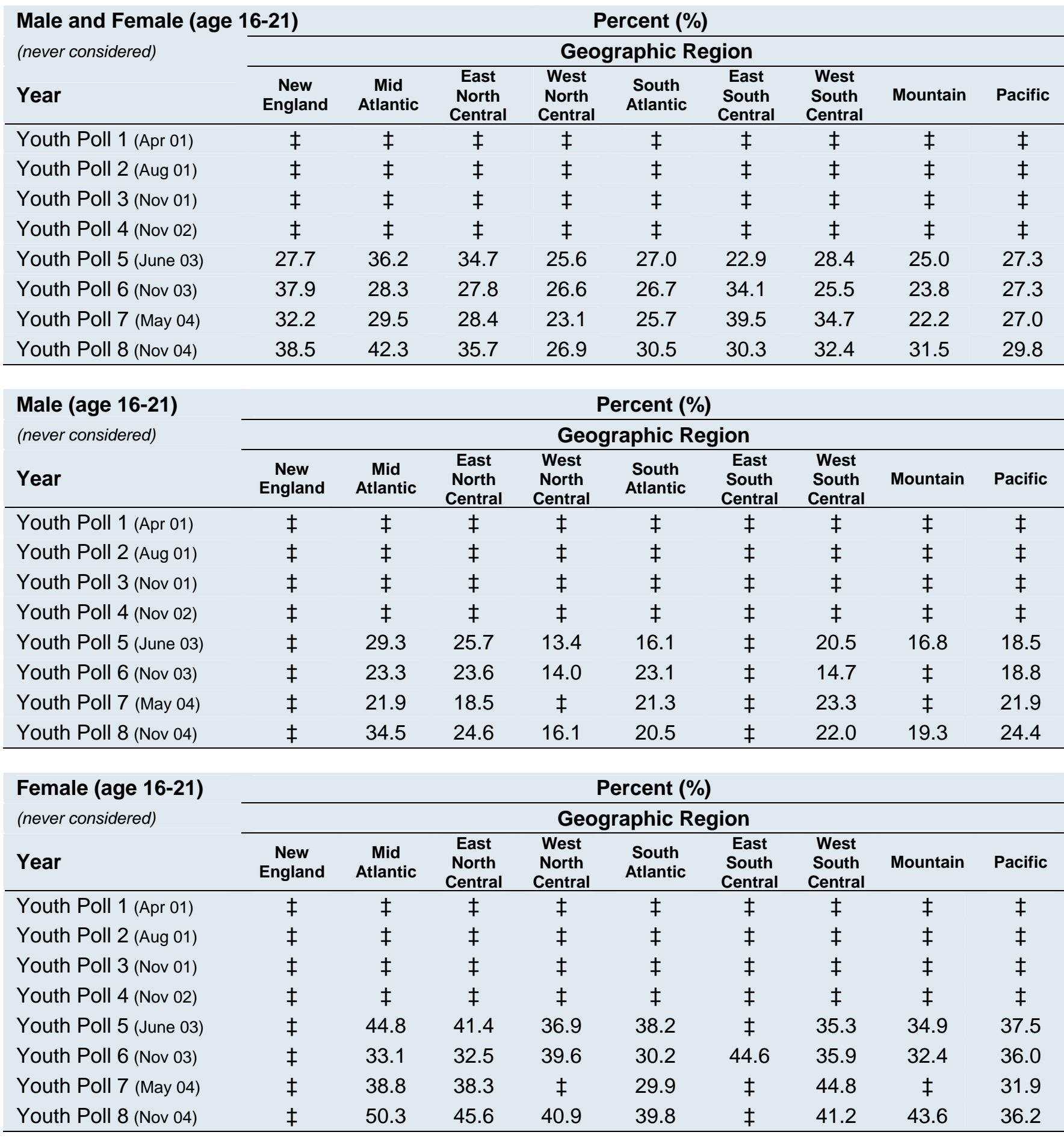

${ }^{54} \ddagger$ Reporting standard not met (too few cases).

Note: Changes in sample size and stratification were implemented following November 2002 Youth Poll.

Source: Department of Defense Polls, JAMRS, 2001-2004 (Question: FPP15).

Page A-54 DoD May 2004 Youth Poll 


\section{Military Consideration}

JAMRS

TABLE 11-5. Youth consideration of military service, by high school grades: $2001-2004^{55}$



\begin{tabular}{lccccccc} 
Females (age 16-21) & \multicolumn{7}{c}{ High School Grades } \\
\cline { 2 - 7 } (never considered) & $\begin{array}{c}\text { Mostly } \\
\text { A's }\end{array}$ & $\begin{array}{c}\text { Mostly A's } \\
\text { \& B's }\end{array}$ & $\begin{array}{c}\text { Mostly } \\
\text { B's }\end{array}$ & $\begin{array}{c}\text { Mostly B's } \\
\text { \& C's }\end{array}$ & $\begin{array}{c}\text { Mostly } \\
\text { C's }\end{array}$ & $\begin{array}{c}\text { Mostly C's } \\
\text { \& D's }\end{array}$ & $\begin{array}{c}\text { Mostly D's } \\
\text { and Lower }\end{array}$ \\
\cline { 2 - 8 } & 46.5 & 43.8 & $\ddagger$ & 49.9 & $\ddagger$ & $\ddagger$ & $\ddagger$ \\
\hline Youth Poll 1 (Apr 01) & 35.8 & 40.4 & 24.8 & 39.7 & $\ddagger$ & $\ddagger$ & $\ddagger$ \\
Youth Poll 2 (Aug 01) & 35.8 & 37.8 & 44.0 & 27.5 & $\ddagger$ & $\ddagger$ & $\ddagger$ \\
Youth Poll 3 (Nov 01) & 45.4 & 37.0 & 36.1 & 34.4 & $\ddagger$ & $\ddagger$ & $\ddagger$ \\
Youth Poll 4 (Nov 02) & 38.3 & 35.1 & 40.1 & 35.2 & $\ddagger$ & $\ddagger$ & $\ddagger$ \\
Youth Poll 5 (June 03) & 48.3 & 34.7 & 34.4 & 35.2 & $\ddagger$ & $\ddagger$ & $\ddagger$ \\
Youth Poll 6 (Nov 03) & 40.4 & 34.7 & & $\ddagger$ \\
Youth Poll 7 (May 04) & 41.9 & 34.0 & 36.7 & 34.7 & $\ddagger$ & $\ddagger$ & $\ddagger$ \\
Youth Poll 8 (Nov 04) & 40.9 & 43.9 & 37.2 & 49.3 & $\ddagger$ & $\ddagger$ & $\ddagger$ \\
\hline
\end{tabular}

${ }^{55}$ ‡Reporting standard not met (too few cases).

Note: Changes in sample size and stratification were implemented following November 2002 Youth Poll.

Source: Department of Defense Polls, JAMRS, 2001-2004 (Question: FPP15). 


\section{U.S. Military Favorability}

TABLE 12-1. Youth U.S. military favorability: $2001-2004^{56}$

\begin{tabular}{lc}
$\begin{array}{l}\text { Male and Female (age 16-21) } \\
\text { (mean) }\end{array}$ & \\
\cline { 2 - 2 } Year & Mean \\
\hline Youth Poll 1 (Apr 01) & QNA \\
Youth Poll 2 (Aug 01) & QNA \\
Youth Poll 3 (Nov 01) & 8.4 \\
Youth Poll 4 (Nov 02) & 7.3 \\
Youth Poll 5 (June 03) & 7.8 \\
Youth Poll 6 (Nov 03) & 7.4 \\
Youth Poll 7 (May 04) & 7.3 \\
Youth Poll 8 (Nov 04) & 7.2 \\
\hline
\end{tabular}

\begin{tabular}{lc}
$\begin{array}{l}\text { Male (age 16-21) } \\
\text { (mean) }\end{array}$ \\
\cline { 2 - 2 } Year & Mean \\
\hline Youth Poll 1 (Apr 01) & QNA \\
Youth Poll 2 (Aug 01) & QNA \\
Youth Poll 3 (Nov 01) & 8.4 \\
Youth Poll 4 (Nov 02) & 7.3 \\
Youth Poll 5 (June 03) & 7.8 \\
Youth Poll 6 (Nov 03) & 7.4 \\
Youth Poll 7 (May 04) & 7.4 \\
Youth Poll 8 (Nov 04) & 7.4 \\
\hline
\end{tabular}

\begin{tabular}{lc}
$\begin{array}{l}\text { Female (age 16-21) } \\
\text { (mean) }\end{array}$ & \\
\cline { 2 - 2 } Year & Mean \\
\hline Youth Poll 1 (Apr 01) & QNA \\
Youth Poll 2 (Aug 01) & QNA \\
Youth Poll 3 (Nov 01) & 8.4 \\
Youth Poll 4 (Nov 02) & 7.3 \\
Youth Poll 5 (June 03) & 7.8 \\
Youth Poll 6 (Nov 03) & 7.3 \\
Youth Poll 7 (May 04) & 7.2 \\
Youth Poll 8 (Nov 04) & 7.1 \\
\hline
\end{tabular}

${ }^{56}$ ‡Reporting standard not met (too few cases); QNA: Question Not Asked Note: Changes in sample size and stratification were implemented following November 2002 Youth Poll. Source: Department of Defense Polls, JAMRS, 2001-2004 (Question: FAV1). 


\section{U.S. Military Favorability}

TABLE 12-2. Youth U.S. military favorability, by race/ethnicity: $2001-2004^{57}$

\begin{tabular}{lccc} 
Male and Female (age 16-21) & \multicolumn{3}{c}{ Racelethnicity } \\
\cline { 2 - 4 } (mean) & White & Black & Hispanic \\
\cline { 2 - 4 } Year & QNA & QNA & QNA \\
\hline Youth Poll 1 (Apr 01) & QNA & QNA & QNA \\
Youth Poll 2 (Aug 01) & 8.4 & 8.5 & 8.3 \\
Youth Poll 3 (Nov 01) & 7.4 & 7.0 & 7.2 \\
Youth Poll 4 (Nov 02) & 7.9 & 7.0 & 8.0 \\
Youth Poll 5 (June 03) & 7.5 & 6.9 & 7.4 \\
Youth Poll 6 (Nov 03) & 7.5 & 6.4 & 7.3 \\
Youth Poll 7 (May 04) & 7.5 & 6.1 & 7.3 \\
Youth Poll 8 (Nov 04) & & & \\
\hline
\end{tabular}

\begin{tabular}{lccc} 
Male (age 16-21) & \multicolumn{3}{c}{ Racelethnicity } \\
\cline { 2 - 4 } (mean) & White & Black & Hispanic \\
\cline { 2 - 4 } Year & QNA & QNA & QNA \\
\hline Youth Poll 1 (Apr 01) & QNA & QNA & QNA \\
Youth Poll 2 (Aug 01) & 8.3 & 8.6 & 8.4 \\
Youth Poll 3 (Nov 01) & 7.4 & 7.2 & 7.2 \\
Youth Poll 4 (Nov 02) & 7.9 & 7.1 & 8.0 \\
Youth Poll 5 (June 03) & 7.6 & 6.9 & 7.3 \\
Youth Poll 6 (Nov 03) & 7.6 & 6.8 & 7.3 \\
Youth Poll 7 (May 04) & 7.7 & 6.2 & 7.4 \\
Youth Poll 8 (Nov 04) & & & \\
\hline
\end{tabular}

\begin{tabular}{lccc} 
Female (age 16-21) & \multicolumn{3}{c}{ Racelethnicity } \\
\cline { 2 - 4 } (mean) & White & Black & Hispanic \\
\cline { 2 - 4 } Year & QNA & QNA & QNA \\
\hline Youth Poll 1 (Apr 01) & QNA & QNA & QNA \\
Youth Poll 2 (Aug 01) & 8.4 & 8.4 & 8.1 \\
Youth Poll 3 (Nov 01) & 7.4 & 6.9 & 7.2 \\
Youth Poll 4 (Nov 02) & 8.0 & 6.9 & 8.1 \\
Youth Poll 5 (June 03) & 7.5 & 6.8 & 7.4 \\
Youth Poll 6 (Nov 03) & 7.4 & 6.0 & 7.3 \\
Youth Poll 7 (May 04) & 7.4 & 6.0 & 7.2 \\
Youth Poll 8 (Nov 04) & & &
\end{tabular}

\footnotetext{
${ }^{57}$ Due to relatively small sample sizes, American Indian, Alaska Native, Asian, and Pacific Islander are included in the total but are not shown separately. Black includes African American, and Hispanic includes Latino. Race categories exclude Hispanic origin unless specified otherwise.

‡Reporting standard not met (too few cases); QNA: Question Not Asked

Note: Changes in sample size and stratification were implemented following November 2002 Youth Poll.

Source: Department of Defense Polls, JAMRS, 2001-2004 (Question: FAV1).
} 


\section{U.S. Military Favorability}

JAMRS

TABLE 12-3. Youth U.S. military favorability, by age: $2001-2004^{58}$

\begin{tabular}{lcccccc} 
Male and Female (age 16-21) & \multicolumn{7}{c}{ Age } \\
\cline { 2 - 7 } (mean) & $\mathbf{1 6}$ & $\mathbf{1 7}$ & $\mathbf{1 8}$ & $\mathbf{1 9}$ & $\mathbf{2 0}$ & $\mathbf{2 1}$ \\
\cline { 2 - 7 } Year & QNA & QNA & QNA & QNA & QNA & QNA \\
\cline { 2 - 7 } Youth Poll 1 (Apr 01) & QNA & QNA & QNA & QNA & QNA & QNA \\
Youth Poll 2 (Aug 01) & 8.4 & 8.2 & 8.2 & 8.8 & 8.3 & 8.3 \\
Youth Poll 3 (Nov 01) & 7.4 & 7.4 & 7.1 & 7.3 & 7.1 & 7.5 \\
Youth Poll 4 (Nov 02) & 7.9 & 7.8 & 7.8 & 7.7 & 7.7 & 7.8 \\
Youth Poll 5 (June 03) & 7.5 & 7.3 & 7.5 & 7.5 & 7.2 & 7.3 \\
Youth Poll 6 (Nov 03) & 7.5 & 7.4 & 7.3 & 7.3 & 7.0 & 7.1 \\
Youth Poll 7 (May 04) & 7.2 & 7.2 & 7.4 & 7.1 & 7.2 & 7.3 \\
Youth Poll 8 (Nov 04) & & & & & &
\end{tabular}

\begin{tabular}{lcccccc} 
Male (age 16-21) & \multicolumn{7}{c}{ Age } \\
\cline { 2 - 7 } (mean) & $\mathbf{1 6}$ & $\mathbf{1 7}$ & $\mathbf{1 8}$ & $\mathbf{1 9}$ & $\mathbf{2 0}$ & $\mathbf{2 1}$ \\
\cline { 2 - 7 } Year & QNA & QNA & QNA & QNA & QNA & QNA \\
\hline Youth Poll 1 (Apr 01) & QNA & QNA & QNA & QNA & QNA & QNA \\
Youth Poll 2 (Aug 01) & 8.6 & 8.2 & 8.1 & 8.8 & 8.2 & 8.3 \\
Youth Poll 3 (Nov 01) & 7.5 & 7.6 & 7.1 & 7.1 & 7.2 & 7.5 \\
Youth Poll 4 (Nov 02) & 7.9 & 7.8 & 7.8 & 7.9 & 7.5 & 7.9 \\
Youth Poll 5 (June 03) & 7.6 & 7.4 & 7.4 & 7.7 & 7.1 & 7.4 \\
Youth Poll 6 (Nov 03) & 7.6 & 7.7 & 7.3 & 7.3 & 7.1 & 7.5 \\
Youth Poll 7 (May 04) & 7.5 & 7.3 & 7.7 & 7.2 & 7.4 & 7.3 \\
Youth Poll 8 (Nov 04) & & & & & & 7.3 \\
\hline
\end{tabular}

\section{Female (age 16-21)}

(mean)

Year

Youth Poll 1 (Apr 01)

Youth Poll 2 (Aug 01)

Youth Poll 3 (Nov 01)

Youth Poll 4 (Nov 02)

Youth Poll 5 (June 03)

Youth Poll 6 (Nov 03)

Youth Poll 7 (May 04)

\begin{tabular}{cccccc}
\hline \multicolumn{7}{c}{ Age } \\
\hline $\mathbf{1 6}$ & $\mathbf{1 7}$ & $\mathbf{1 8}$ & $\mathbf{1 9}$ & $\mathbf{2 0}$ & $\mathbf{2 1}$ \\
QNA & QNA & QNA & QNA & QNA & QNA \\
QNA & QNA & QNA & QNA & QNA & QNA \\
8.3 & 8.1 & 8.4 & 8.8 & 8.3 & 8.3 \\
7.3 & 7.2 & 7.1 & 7.5 & 7.0 & 7.5 \\
8.0 & 7.9 & 7.8 & 7.5 & 8.0 & 7.6 \\
7.5 & 7.3 & 7.5 & 7.3 & 7.2 & 7.2 \\
7.5 & 7.0 & 7.4 & 7.3 & 7.0 & 6.8 \\
7.0 & 7.1 & 7.1 & 7.0 & 6.9 & 7.3 \\
\hline
\end{tabular}

Youth Poll 8 (Nov 04)

${ }^{58}$ ‡Reporting standard not met (too few cases); QNA: Question Not Asked

Note: Changes in sample size and stratification were implemented following November 2002 Youth Poll.

Source: Department of Defense Polls, JAMRS, 2001-2004 (Question: FAV1).

Page A-58 DoD May 2004 Youth Poll 


\section{U.S. Military Favorability}

JAMRS

TABLE 12-4. Youth U.S. military favorability, by geographic region: $2001-2004^{59}$

\section{Male and Female (age 16-21)}

(mean)

Year

Youth Poll 1 (Apr 01)

Youth Poll 2 (Aug 01)

Youth Poll 3 (Nov 01)

Youth Poll 4 (Nov 02)

Youth Poll 5 (June 03)



Youth Poll 6 (Nov 03)

New
England

Mid East

QNA

Atlantic

North
Central

QNA QNA

QNA QNA

QNA

\section{Geographic Region}

Youth Poll 7 (May 04)



Youth Poll 8 (Nov 04)



7.6

7.7

$\ddagger$

7.3

7.3

7.8

North

Central

QNA

South

East

7.5

7.1

7.3

QNA

Atlantic

South
Central

QNA

West

South

Mountain Pacific

6.6

6.9

7.0

†

$\ddagger$

QNA

QNA

QNA QNA

QNA

QNA

QNA

‡

$\ddagger$

8.0

7.9

$\ddagger$

$\ddagger$

$\ddagger$

$\ddagger$

7.4

7.3

8.0

7.9

$\ddagger$

7.7

Male (age 16-21)

(mean)

7.0

7.6

7.5

7.8

7.5

7.5

7.3

7.7

7.5

7.4

7.3

7.5

7.2

7.3

7.3

7.6

7.1

\begin{tabular}{|c|c|c|c|c|c|c|c|c|c|}
\hline \multirow{2}{*}{$\begin{array}{l}\text { (mean) } \\
\text { Year }\end{array}$} & \multicolumn{9}{|c|}{ Geographic Region } \\
\hline & $\begin{array}{c}\text { New } \\
\text { England }\end{array}$ & $\begin{array}{c}\text { Mid } \\
\text { Atlantic }\end{array}$ & $\begin{array}{c}\text { East } \\
\text { North } \\
\text { Central }\end{array}$ & $\begin{array}{c}\text { West } \\
\text { North } \\
\text { Central }\end{array}$ & $\begin{array}{c}\text { South } \\
\text { Atlantic }\end{array}$ & $\begin{array}{c}\text { East } \\
\text { South } \\
\text { Central }\end{array}$ & $\begin{array}{l}\text { West } \\
\text { South } \\
\text { Central }\end{array}$ & Mountain & Pacific \\
\hline Youth Poll 1 (Apr 01) & QNA & QNA & QNA & QNA & QNA & QNA & QNA & QNA & QNA \\
\hline Youth Poll 2 (Aug 01) & QNA & QNA & QNA & QNA & QNA & QNA & QNA & QNA & QNA \\
\hline Youth Poll 3 (Nov 01) & $\ddagger$ & $\ddagger$ & $\ddagger$ & $\ddagger$ & $\ddagger$ & $\ddagger$ & $\ddagger$ & $\ddagger$ & $\ddagger$ \\
\hline Youth Poll 4 (Nov 02) & $\ddagger$ & $\ddagger$ & $\ddagger$ & $\ddagger$ & $\ddagger$ & $\ddagger$ & $\ddagger$ & $\ddagger$ & $\ddagger$ \\
\hline Youth Poll 5 (June 03) & $\ddagger$ & 7.8 & 7.7 & 8.0 & 8.0 & $\ddagger$ & 7.8 & 7.8 & 7.5 \\
\hline Youth Poll 6 (Nov 03) & $\ddagger$ & 7.5 & 7.3 & 7.2 & 7.3 & $\ddagger$ & 7.8 & $\ddagger$ & 7.2 \\
\hline Youth Poll 7 (May 04) & $\ddagger$ & 7.2 & 7.1 & $\ddagger$ & 7.6 & $\ddagger$ & 7.3 & $\ddagger$ & 7.3 \\
\hline Youth Poll 8 (Nov 04) & $\ddagger$ & 7.2 & 7.3 & 8.0 & 7.7 & $\ddagger$ & 7.5 & 7.8 & 7.0 \\
\hline
\end{tabular}

Female (age 16-21)

(mean)

\section{Year}

Youth Poll 1 (Apr 01)

Youth Poll 2 (Aug 01)

Youth Poll 3 (Nov 01)

Youth Poll 4 (Nov 02)

Youth Poll 5 (June 03)

Youth Poll 6 (Nov 03)

Youth Poll 7 (May 04)

Youth Poll 8 (Nov 04)

\begin{tabular}{ccccccccc}
\hline \multicolumn{10}{c}{ Geographic Region } \\
$\begin{array}{c}\text { New } \\
\text { England }\end{array}$ & $\begin{array}{c}\text { Mid } \\
\text { Atlantic }\end{array}$ & $\begin{array}{c}\text { East } \\
\text { North } \\
\text { Central }\end{array}$ & $\begin{array}{c}\text { West } \\
\text { North } \\
\text { Central }\end{array}$ & $\begin{array}{c}\text { South } \\
\text { Atlantic }\end{array}$ & $\begin{array}{c}\text { East } \\
\text { South } \\
\text { Central }\end{array}$ & $\begin{array}{c}\text { West } \\
\text { South } \\
\text { Central }\end{array}$ & Mountain & Pacific \\
QNA & QNA & QNA & QNA & QNA & QNA & QNA & QNA & QNA \\
QNA & QNA & QNA & QNA & QNA & QNA & QNA & QNA & QNA \\
$\ddagger$ & $\ddagger$ & $\ddagger$ & $\ddagger$ & $\ddagger$ & $\ddagger$ & $\ddagger$ & $\ddagger$ & $\ddagger$ \\
$\ddagger$ & $\ddagger$ & $\ddagger$ & $\ddagger$ & $\ddagger$ & $\ddagger$ & $\ddagger$ & $\ddagger$ & $\ddagger$ \\
$\ddagger$ & 7.5 & 7.8 & 8.1 & 7.8 & $\ddagger$ & 8.0 & 7.7 & 7.8 \\
$\ddagger$ & 7.2 & 7.4 & 7.5 & 7.4 & 7.6 & 7.2 & 7.5 & 7.4 \\
$\ddagger$ & 7.0 & 6.8 & $\ddagger$ & 7.4 & $\ddagger$ & 7.2 & $\ddagger$ & 7.1 \\
$\ddagger$ & 6.5 & 6.8 & 7.2 & 7.3 & $\ddagger$ & 7.2 & 7.5 & 7.2 \\
\hline
\end{tabular}

${ }^{59}$ ‡Reporting standard not met (too few cases); QNA: Question Not Asked

Note: Changes in sample size and stratification were implemented following November 2002 Youth Poll.

Source: Department of Defense Polls, JAMRS, 2001-2004 (Question: FAV1).

Page A-59 DoD May 2004 Youth Poll 


\section{U.S. Military Favorability}

JAMRS

TABLE 12-5. Youth U.S. military favorability, by high school grades: $2001-2004^{60}$

\begin{tabular}{|c|c|c|c|c|c|c|c|}
\hline \multicolumn{8}{|c|}{ Male and Female (age 16-21) } \\
\hline \multirow{2}{*}{$\begin{array}{l}\text { (mean) } \\
\text { Year }\end{array}$} & \multicolumn{7}{|c|}{ High School Grades } \\
\hline & $\begin{array}{l}\text { Mostly } \\
\text { A's }\end{array}$ & $\begin{array}{l}\text { Mostly A's } \\
\text { \& B's }\end{array}$ & $\begin{array}{l}\text { Mostly } \\
\text { B's }\end{array}$ & $\begin{array}{l}\text { Mostly B's } \\
\text { \& C's }\end{array}$ & $\begin{array}{l}\text { Mostly } \\
\text { C's }\end{array}$ & $\begin{array}{l}\text { Mostly C's } \\
\text { \& D's }\end{array}$ & $\begin{array}{l}\text { Mostly D's } \\
\text { and Lower }\end{array}$ \\
\hline Youth Poll 1 (Apr 01) & QNA & QNA & QNA & QNA & QNA & QNA & QNA \\
\hline Youth Poll 2 (Aug 01) & QNA & QNA & QNA & QNA & QNA & QNA & QNA \\
\hline Youth Poll 3 (Nov 01) & 7.9 & 8.4 & 8.4 & 8.5 & $\ddagger$ & $\ddagger$ & $\ddagger$ \\
\hline Youth Poll 4 (Nov 02) & 7.5 & 7.3 & 7.2 & 7.4 & 6.9 & $\ddagger$ & $\ddagger$ \\
\hline Youth Poll 5 (June 03) & 7.8 & 8.0 & 7.7 & 7.8 & 7.2 & 7.9 & $\ddagger$ \\
\hline Youth Poll 6 (Nov 03) & 7.3 & 7.4 & 7.1 & 7.6 & 7.0 & 7.6 & $\ddagger$ \\
\hline Youth Poll 7 (May 04) & 7.5 & 7.3 & 7.3 & 7.3 & 6.8 & 7.1 & $\ddagger$ \\
\hline Youth Poll 8 (Nov 04) & 7.6 & 7.3 & 7.0 & 7.1 & 7.1 & 7.0 & $\ddagger$ \\
\hline \multicolumn{8}{|l|}{ Male (age 16-21) } \\
\hline (mean) & \multicolumn{7}{|c|}{ High School Grades } \\
\hline Year & $\begin{array}{c}\text { Mostly } \\
\text { A's }\end{array}$ & $\begin{array}{c}\text { Mostly A's } \\
\text { \& B's }\end{array}$ & $\begin{array}{l}\text { Mostly } \\
\text { B's }\end{array}$ & $\begin{array}{l}\text { Mostly B's } \\
\text { \& C's }\end{array}$ & $\begin{array}{l}\text { Mostly } \\
\text { C's }\end{array}$ & $\begin{array}{l}\text { Mostly C's } \\
\text { \& D's }\end{array}$ & $\begin{array}{l}\text { Mostly D's } \\
\text { and Lower }\end{array}$ \\
\hline Youth Poll 1 (Apr 01) & QNA & QNA & QNA & QNA & QNA & QNA & QNA \\
\hline Youth Poll 2 (Aug 01) & QNA & QNA & QNA & QNA & QNA & QNA & QNA \\
\hline Youth Poll 3 (Nov 01) & 8.0 & 8.4 & 8.5 & 8.5 & $\ddagger$ & $\ddagger$ & $\ddagger$ \\
\hline Youth Poll 4 (Nov 02) & $\ddagger$ & 7.3 & 7.2 & 7.4 & $\ddagger$ & $\ddagger$ & $\ddagger$ \\
\hline Youth Poll 5 (June 03) & 8.0 & 7.9 & 7.9 & 7.8 & 7.0 & 7.7 & $\ddagger$ \\
\hline Youth Poll 6 (Nov 03) & 7.0 & 7.6 & 7.4 & 7.6 & 7.0 & 7.8 & $\ddagger$ \\
\hline Youth Poll 7 (May 04) & 7.4 & 7.4 & 7.3 & 7.7 & $\ddagger$ & 7.5 & $\ddagger$ \\
\hline Youth Poll 8 (Nov 04) & 7.8 & 7.5 & 7.1 & 7.3 & 7.4 & $\ddagger$ & $\ddagger$ \\
\hline
\end{tabular}

Females (age 16-21)

(mean)

High School Grades

\begin{tabular}{lccccccc}
\cline { 2 - 7 } Year & $\begin{array}{c}\text { Mostly } \\
\text { A's }\end{array}$ & $\begin{array}{c}\text { Mostly A's } \\
\text { \& B's }\end{array}$ & $\begin{array}{c}\text { Mostly } \\
\text { B's }\end{array}$ & $\begin{array}{c}\text { Mostly B's } \\
\text { \& C's }\end{array}$ & $\begin{array}{c}\text { Mostly } \\
\text { C's }\end{array}$ & $\begin{array}{c}\text { Mostly C's } \\
\text { \& D's }\end{array}$ & $\begin{array}{c}\text { Mostly D's } \\
\text { and Lower }\end{array}$ \\
\hline Youth Poll 1 (Apr 01) & QNA & QNA & QNA & QNA & QNA & QNA & QNA \\
Youth Poll 2 (Aug 01) & QNA & QNA & QNA & QNA & QNA & QNA & QNA \\
Youth Poll 3 (Nov 01) & 7.9 & 8.5 & 8.3 & 8.6 & $\ddagger$ & $\ddagger$ & $\ddagger$ \\
Youth Poll 4 (Nov 02) & 7.4 & 7.3 & 7.2 & 7.3 & $\ddagger$ & $\ddagger$ & $\ddagger$ \\
Youth Poll 5 (June 03) & 7.6 & 8.1 & 7.5 & 7.7 & $\ddagger$ & $\ddagger$ & $\ddagger$ \\
Youth Poll 6 (Nov 03) & 7.5 & 7.3 & 6.9 & 7.6 & $\ddagger$ & $\ddagger$ & $\ddagger$ \\
Youth Poll 7 (May 04) & 7.5 & 7.3 & 7.3 & 6.8 & $\ddagger$ & $\ddagger$ & $\ddagger$ \\
Youth Poll 8 (Nov 04) & 7.5 & 7.1 & 6.8 & 6.9 & $\ddagger$ & $\ddagger$ & $\ddagger$ \\
\hline
\end{tabular}

${ }^{60} \ddagger$ Reporting standard not met (too few cases); QNA: Question Not Asked

Note: Changes in sample size and stratification were implemented following November 2002 Youth Poll.

Source: Department of Defense Polls, JAMRS, 2001-2004 (Question: FAV1). 


\section{Army Favorability}

TABLE 13-1. Youth Army favorability: $2001-2004^{61}$

\begin{tabular}{lc}
$\begin{array}{l}\text { Male and Female (age 16-21) } \\
\text { (mean) }\end{array}$ & \\
\cline { 2 - 2 } Year & Mean \\
\hline Youth Poll 1 (Apr 01) & QNA \\
Youth Poll 2 (Aug 01) & QNA \\
Youth Poll 3 (Nov 01) & 8.3 \\
Youth Poll 4 (Nov 02) & 7.1 \\
Youth Poll 5 (June 03) & 7.5 \\
Youth Poll 6 (Nov 03) & 7.2 \\
Youth Poll 7 (May 04) & 7.0 \\
Youth Poll 8 (Nov 04) & 7.1 \\
\hline
\end{tabular}

\begin{tabular}{lc}
$\begin{array}{l}\text { Male (age 16-21) } \\
\text { (mean) }\end{array}$ \\
\cline { 2 - 2 } Year & Mean \\
\hline Youth Poll 1 (Apr 01) & QNA \\
Youth Poll 2 (Aug 01) & QNA \\
Youth Poll 3 (Nov 01) & 8.1 \\
Youth Poll 4 (Nov 02) & 6.9 \\
Youth Poll 5 (June 03) & 7.4 \\
Youth Poll 6 (Nov 03) & 7.1 \\
Youth Poll 7 (May 04) & 7.0 \\
Youth Poll 8 (Nov 04) & 7.1 \\
\hline
\end{tabular}

\begin{tabular}{lc}
$\begin{array}{l}\text { Female (age 16-21) } \\
\text { (mean) }\end{array}$ & \\
\cline { 2 - 2 } Year & Mean \\
\hline Youth Poll 1 (Apr 01) & QNA \\
Youth Poll 2 (Aug 01) & QNA \\
Youth Poll 3 (Nov 01) & 8.4 \\
Youth Poll 4 (Nov 02) & 7.3 \\
Youth Poll 5 (June 03) & 7.7 \\
Youth Poll 6 (Nov 03) & 7.4 \\
Youth Poll 7 (May 04) & 7.0 \\
Youth Poll 8 (Nov 04) & 7.1 \\
\hline
\end{tabular}

${ }^{61} \ddagger$ Reporting standard not met (too few cases); QNA: Question Not Asked Note: Changes in sample size and stratification were implemented following November 2002 Youth Poll. Source: Department of Defense Polls, JAMRS, 2001-2004 (Question: FAV2B). 


\section{Army Favorability}

TABLE 13-2. Youth Army favorability, by race/ethnicity: $2001-2004^{62}$

\begin{tabular}{lccc} 
Male and Female (age 16-21) & \multicolumn{3}{c}{ Race/ethnicity } \\
\cline { 2 - 4 } (mean) & White & Black & Hispanic \\
\cline { 2 - 4 } Year & QNA & QNA & QNA \\
\hline Youth Poll 1 (Apr 01) & QNA & QNA & QNA \\
Youth Poll 2 (Aug 01) & 8.2 & 8.6 & 8.2 \\
Youth Poll 3 (Nov 01) & 7.2 & 6.9 & 7.0 \\
Youth Poll 4 (Nov 02) & 7.7 & 6.8 & 7.6 \\
Youth Poll 5 (June 03) & 7.3 & 6.8 & 7.4 \\
Youth Poll 6 (Nov 03) & 7.2 & 6.3 & 7.1 \\
Youth Poll 7 (May 04) & 7.3 & 6.0 & 7.2 \\
Youth Poll 8 (Nov 04) & & &
\end{tabular}

\begin{tabular}{lccc} 
Male (age 16-21) & \multicolumn{3}{c}{ Racelethnicity } \\
\cline { 2 - 4 } (mean) & White & Black & Hispanic \\
\cline { 2 - 4 } Year & QNA & QNA & QNA \\
\hline Youth Poll 1 (Apr 01) & QNA & QNA & QNA \\
Youth Poll 2 (Aug 01) & 8.0 & 8.7 & 8.2 \\
Youth Poll 3 (Nov 01) & 7.0 & 7.0 & 6.8 \\
Youth Poll 4 (Nov 02) & 7.5 & 6.7 & 7.4 \\
Youth Poll 5 (June 03) & 7.2 & 6.7 & 7.1 \\
Youth Poll 6 (Nov 03) & 7.1 & 6.5 & 7.2 \\
Youth Poll 7 (May 04) & 7.3 & 6.1 & 7.1 \\
Youth Poll 8 (Nov 04) & & & \\
\hline
\end{tabular}

\begin{tabular}{lccc} 
Female (age 16-21) & \multicolumn{3}{c}{ Racelethnicity } \\
\cline { 2 - 4 } (mean) & White & Black & Hispanic \\
\cline { 2 - 4 } Year & QNA & QNA & QNA \\
\hline Youth Poll 1 (Apr 01) & QNA & QNA & QNA \\
Youth Poll 2 (Aug 01) & 8.4 & 8.5 & 8.3 \\
Youth Poll 3 (Nov 01) & 7.3 & 6.8 & 7.1 \\
Youth Poll 4 (Nov 02) & 7.9 & 6.9 & 7.8 \\
Youth Poll 5 (June 03) & 7.4 & 6.8 & 7.7 \\
Youth Poll 6 (Nov 03) & 7.2 & 6.2 & 7.0 \\
Youth Poll 7 (May 04) & 7.3 & 6.0 & 7.3 \\
Youth Poll 8 (Nov 04) & & & \\
\hline
\end{tabular}

${ }^{62}$ Due to relatively small sample sizes, American Indian, Alaska Native, Asian, and Pacific Islander are included in the total but are not shown separately. Black includes African American, and Hispanic includes Latino. Race categories exclude Hispanic origin unless specified otherwise.

†Reporting standard not met (too few cases); QNA: Question Not Asked

Note: Changes in sample size and stratification were implemented following November 2002 Youth Poll.

Source: Department of Defense Polls, JAMRS, 2001-2004 (Question: FAV2B). 


\section{Army Favorability}

JAMRS

TABLE 13-3. Youth Army favorability, by age: $2001-2004^{63}$

\begin{tabular}{lcccccc} 
Male and Female (age 16-21) & \multicolumn{7}{c}{ Age } \\
\cline { 2 - 7 } (mean) & 16 & $\mathbf{1 7}$ & $\mathbf{1 8}$ & $\mathbf{1 9}$ & $\mathbf{2 0}$ & $\mathbf{2 1}$ \\
\cline { 2 - 7 } Year & QNA & QNA & QNA & QNA & QNA & QNA \\
\cline { 2 - 7 } Youth Poll 1 (Apr 01) & QNA & QNA & QNA & QNA & QNA & QNA \\
Youth Poll 2 (Aug 01) & 8.3 & 8.1 & 8.2 & 8.5 & 8.3 & 8.3 \\
Youth Poll 3 (Nov 01) & 7.3 & 7.1 & 6.8 & 7.2 & 6.8 & 7.4 \\
Youth Poll 4 (Nov 02) & 7.7 & 7.6 & 7.5 & 7.6 & 7.3 & 7.6 \\
Youth Poll 5 (June 03) & 7.4 & 7.2 & 7.2 & 7.3 & 7.3 & 7.0 \\
Youth Poll 6 (Nov 03) & 7.3 & 7.1 & 7.0 & 7.2 & 6.9 & 6.8 \\
Youth Poll 7 (May 04) & 7.3 & 7.0 & 7.2 & 6.8 & 7.0 & 7.2 \\
Youth Poll 8 (Nov 04) & & & & & & 7.0 \\
\hline
\end{tabular}

\begin{tabular}{lcccccc} 
Male (age 16-21) & \multicolumn{7}{c}{ Age } \\
\cline { 2 - 7 } (mean) & $\mathbf{1 6}$ & $\mathbf{1 7}$ & $\mathbf{1 8}$ & $\mathbf{1 9}$ & $\mathbf{2 0}$ & $\mathbf{2 1}$ \\
\cline { 2 - 7 } Year & QNA & QNA & QNA & QNA & QNA & QNA \\
\hline Youth Poll 1 (Apr 01) & QNA & QNA & QNA & QNA & QNA & QNA \\
Youth Poll 2 (Aug 01) & 8.3 & 8.1 & 8.0 & 8.2 & 8.0 & 8.3 \\
Youth Poll 3 (Nov 01) & 7.3 & 7.1 & 6.7 & 6.7 & 6.6 & 7.3 \\
Youth Poll 4 (Nov 02) & 7.6 & 7.4 & 7.4 & 7.6 & 6.9 & 7.6 \\
Youth Poll 5 (June 03) & 7.3 & 7.0 & 7.1 & 7.1 & 7.1 & 6.9 \\
Youth Poll 6 (Nov 03) & 7.3 & 7.2 & 6.8 & 7.1 & 6.7 & 7.0 \\
Youth Poll 7 (May 04) & 7.3 & 6.9 & 7.4 & 6.8 & 7.1 & 7.2 \\
Youth Poll 8 (Nov 04) & & & & & & 7.2 \\
\hline
\end{tabular}

\section{Female (age 16-21)}

(mean)

\begin{tabular}{cccccc}
\hline \multicolumn{7}{c}{ Age } \\
\hline $\mathbf{1 6}$ & $\mathbf{1 7}$ & $\mathbf{1 8}$ & $\mathbf{1 9}$ & $\mathbf{2 0}$ & $\mathbf{2 1}$ \\
QNA & QNA & QNA & QNA & QNA & QNA \\
QNA & QNA & QNA & QNA & QNA & QNA \\
8.3 & 8.1 & 8.4 & 8.7 & 8.6 & 8.3 \\
7.4 & 7.2 & 6.9 & 7.6 & 6.9 & 7.4 \\
7.8 & 7.8 & 7.6 & 7.6 & 7.7 & 7.6 \\
7.5 & 7.3 & 7.3 & 7.4 & 7.6 & 7.1 \\
7.3 & 6.9 & 7.3 & 7.2 & 7.0 & 6.6 \\
7.2 & 7.1 & 7.0 & 6.9 & 6.9 & 7.2 \\
\hline
\end{tabular}

${ }^{63}$ ‡Reporting standard not met (too few cases); QNA: Question Not Asked Note: Changes in sample size and stratification were implemented following November 2002 Youth Poll. Source: Department of Defense Polls, JAMRS, 2001-2004 (Question: FAV2B).

Page A-63 DoD May 2004 Youth Poll 


\section{Army Favorability}

JAMRS

TABLE 13-4. Youth Army favorability, by geographic region: $2001-2004^{64}$

\section{Male and Female (age 16-21)}

(mean)

Year

Youth Poll 1 (Apr 01)

Youth Poll 2 (Aug 01)

Youth Poll 3 (Nov 01)

Youth Poll 4 (Nov 02)

Youth Poll 5 (June 03)

\begin{tabular}{ccccccccc}
\hline \multicolumn{10}{c}{ Geographic Region } \\
\hline $\begin{array}{c}\text { New } \\
\text { England }\end{array}$ & $\begin{array}{c}\text { Mid } \\
\text { Atlantic }\end{array}$ & $\begin{array}{c}\text { East } \\
\text { North } \\
\text { Central }\end{array}$ & $\begin{array}{c}\text { West } \\
\text { North } \\
\text { Central }\end{array}$ & $\begin{array}{c}\text { South } \\
\text { Atlantic }\end{array}$ & $\begin{array}{c}\text { East } \\
\text { South } \\
\text { Central }\end{array}$ & $\begin{array}{c}\text { West } \\
\text { South } \\
\text { Central }\end{array}$ & Mountain & Pacific \\
QNA & QNA & QNA & QNA & QNA & QNA & QNA & QNA & QNA \\
QNA & QNA & QNA & QNA & QNA & QNA & QNA & QNA & QNA \\
$\ddagger$ & $\ddagger$ & $\ddagger$ & $\ddagger$ & $\ddagger$ & $\ddagger$ & $\ddagger$ & $\ddagger$ & $\ddagger$ \\
$\ddagger$ & $\ddagger$ & $\ddagger$ & $\ddagger$ & $\ddagger$ & $\ddagger$ & $\ddagger$ & $\ddagger$ & $\ddagger$ \\
7.5 & 7.6 & 7.6 & 7.7 & 7.7 & 7.5 & 7.6 & 7.5 & 7.3 \\
7.2 & 7.3 & 7.3 & 7.3 & 7.2 & 7.6 & 7.1 & 7.3 & 7.0 \\
7.3 & 7.0 & 7.1 & 7.2 & 7.2 & 7.1 & 6.9 & 7.0 & 6.8 \\
6.5 & 6.7 & 6.8 & 7.6 & 7.3 & 7.2 & 7.3 & 7.4 & 6.9 \\
\hline
\end{tabular}

Youth Poll 6 (Nov 03)

Youth Poll 7 (May 04)

6.7

\begin{tabular}{ccccccccc}
\hline \multicolumn{10}{c}{ Geographic Region } \\
\hline $\begin{array}{c}\text { New } \\
\text { England }\end{array}$ & $\begin{array}{c}\text { Mid } \\
\text { Atlantic }\end{array}$ & $\begin{array}{c}\text { East } \\
\text { North } \\
\text { Central }\end{array}$ & $\begin{array}{c}\text { West } \\
\text { North } \\
\text { Central }\end{array}$ & $\begin{array}{c}\text { South } \\
\text { Atlantic }\end{array}$ & $\begin{array}{c}\text { East } \\
\text { South } \\
\text { Central }\end{array}$ & $\begin{array}{c}\text { West } \\
\text { South } \\
\text { Central }\end{array}$ & Mountain & Pacific \\
QNA & QNA & QNA & QNA & QNA & QNA & QNA & QNA & QNA \\
QNA & QNA & QNA & QNA & QNA & QNA & QNA & QNA & QNA \\
$\ddagger$ & $\ddagger$ & $\ddagger$ & $\ddagger$ & $\ddagger$ & $\ddagger$ & $\ddagger$ & $\ddagger$ & $\ddagger$ \\
$\ddagger$ & $\ddagger$ & $\ddagger$ & $\ddagger$ & $\ddagger$ & $\ddagger$ & $\ddagger$ & $\ddagger$ & $\ddagger$ \\
$\ddagger$ & 7.6 & 7.4 & 7.3 & 7.5 & $\ddagger$ & 7.3 & 7.3 & 7.2 \\
$\ddagger$ & 7.3 & 7.1 & 6.9 & 7.0 & $\ddagger$ & 7.0 & $\ddagger$ & 6.7 \\
$\ddagger$ & 6.7 & 7.3 & $\ddagger$ & 7.3 & $\ddagger$ & 6.9 & $\ddagger$ & 6.9 \\
$\ddagger$ & 6.7 & 6.9 & 7.8 & 7.5 & $\ddagger$ & 7.4 & 7.3 & 6.7 \\
\hline
\end{tabular}

Female (age 16-21)

(mean)

Male (age 16-21)

(mean)

Year

Youth Poll 1 (Apr 01)

Youth Poll 2 (Aug 01)

Youth Poll 3 (Nov 01)

Youth Poll 4 (Nov 02)

Youth Poll 5 (June 03)

Youth Poll 6 (Nov 03)

Youth Poll 7 (May 04)

Geographic Region

\section{Year}

Youth Poll 1 (Apr 01)

Youth Poll 2 (Aug 01)

Youth Poll 3 (Nov 01)

Youth Poll 4 (Nov 02)

Youth Poll 5 (June 03)

Youth Poll 6 (Nov 03)

Youth Poll 7 (May 04)

Youth Poll 8 (Nov 04)

\begin{tabular}{ccccccccc}
\hline \multicolumn{10}{c}{ Geographic Region } \\
$\begin{array}{c}\text { New } \\
\text { England }\end{array}$ & $\begin{array}{c}\text { Mid } \\
\text { Atlantic }\end{array}$ & $\begin{array}{c}\text { East } \\
\text { North } \\
\text { Central }\end{array}$ & $\begin{array}{c}\text { West } \\
\text { North } \\
\text { Central }\end{array}$ & $\begin{array}{c}\text { South } \\
\text { Atlantic }\end{array}$ & $\begin{array}{c}\text { East } \\
\text { South } \\
\text { Central }\end{array}$ & $\begin{array}{c}\text { West } \\
\text { South } \\
\text { Central }\end{array}$ & Mountain & Pacific \\
QNA & QNA & QNA & QNA & QNA & QNA & QNA & QNA & QNA \\
QNA & QNA & QNA & QNA & QNA & QNA & QNA & QNA & QNA \\
$\ddagger$ & $\ddagger$ & $\ddagger$ & $\ddagger$ & $\ddagger$ & $\ddagger$ & $\ddagger$ & $\ddagger$ & $\ddagger$ \\
$\ddagger$ & $\ddagger$ & $\ddagger$ & $\ddagger$ & $\ddagger$ & $\ddagger$ & $\ddagger$ & $\ddagger$ & $\ddagger$ \\
$\ddagger$ & 7.6 & 7.7 & 8.0 & 7.8 & $\ddagger$ & 7.7 & 7.8 & 7.5 \\
$\ddagger$ & 7.4 & 7.4 & 7.6 & 7.5 & 7.4 & 7.2 & 7.4 & 7.3 \\
$\ddagger$ & 7.3 & 6.8 & $\ddagger$ & 7.2 & $\ddagger$ & 7.0 & $\ddagger$ & 6.7 \\
$\ddagger$ & 6.7 & 6.8 & 7.2 & 7.1 & $\ddagger$ & 7.2 & 7.4 & 7.1 \\
\hline
\end{tabular}

${ }^{64}$ ‡Reporting standard not met (too few cases); QNA: Question Not Asked

Note: Changes in sample size and stratification were implemented following November 2002 Youth Poll.

Source: Department of Defense Polls, JAMRS, 2001-2004 (Question: FAV2B). 


\section{Army Favorability}

JAMRS

TABLE 13-5. Youth Army favorability, by high school grades: $2001-2004^{65}$

\begin{tabular}{|c|c|c|c|c|c|c|c|}
\hline \multicolumn{8}{|c|}{ Male and Female (age 16-21) } \\
\hline \multirow{2}{*}{$\begin{array}{l}\text { (mean) } \\
\text { Year }\end{array}$} & \multicolumn{7}{|c|}{ High School Grades } \\
\hline & $\begin{array}{c}\text { Mostly } \\
\text { A's }\end{array}$ & $\begin{array}{l}\text { Mostly A's } \\
\text { \& B's }\end{array}$ & $\begin{array}{l}\text { Mostly } \\
\text { B's }\end{array}$ & $\begin{array}{l}\text { Mostly B's } \\
\text { \& C's }\end{array}$ & $\begin{array}{l}\text { Mostly } \\
\text { C's }\end{array}$ & $\begin{array}{l}\text { Mostly C's } \\
\text { \& D's }\end{array}$ & $\begin{array}{l}\text { Mostly D's } \\
\text { and Lower }\end{array}$ \\
\hline Youth Poll 1 (Apr 01) & QNA & QNA & QNA & QNA & QNA & QNA & QNA \\
\hline Youth Poll 2 (Aug 01) & QNA & QNA & QNA & QNA & QNA & QNA & QNA \\
\hline Youth Poll 3 (Nov 01) & 8.0 & 8.4 & 8.2 & 8.4 & $\ddagger$ & $\ddagger$ & $\ddagger$ \\
\hline Youth Poll 4 (Nov 02) & 7.1 & 7.1 & 7.1 & 7.2 & 6.7 & $\ddagger$ & $\ddagger$ \\
\hline Youth Poll 5 (June 03) & 7.5 & 7.7 & 7.6 & 7.5 & 7.2 & 7.6 & $\ddagger$ \\
\hline Youth Poll 6 (Nov 03) & 7.2 & 7.4 & 7.0 & 7.2 & 6.7 & 7.5 & $\ddagger$ \\
\hline Youth Poll 7 (May 04) & 7.0 & 7.1 & 6.8 & 7.1 & 6.5 & 6.8 & $\ddagger$ \\
\hline Youth Poll 8 (Nov 04) & 7.5 & 7.1 & 6.8 & 7.0 & 7.0 & 6.6 & $\ddagger$ \\
\hline \multicolumn{8}{|l|}{ Male (age 16-21) } \\
\hline (mean) & \multicolumn{7}{|c|}{ High School Grades } \\
\hline Year & $\begin{array}{c}\text { Mostly } \\
\text { A's }\end{array}$ & $\begin{array}{l}\text { Mostly A's } \\
\text { \& B's }\end{array}$ & $\begin{array}{l}\text { Mostly } \\
\text { B's }\end{array}$ & $\begin{array}{l}\text { Mostly B's } \\
\text { \& C's }\end{array}$ & $\begin{array}{l}\text { Mostly } \\
\text { C's }\end{array}$ & $\begin{array}{l}\text { Mostly C's } \\
\text { \& D's }\end{array}$ & $\begin{array}{l}\text { Mostly D's } \\
\text { and Lower }\end{array}$ \\
\hline Youth Poll 1 (Apr 01) & QNA & QNA & QNA & QNA & QNA & QNA & QNA \\
\hline Youth Poll 2 (Aug 01) & QNA & QNA & QNA & QNA & QNA & QNA & QNA \\
\hline Youth Poll 3 (Nov 01) & 7.9 & 8.2 & 8.1 & 8.2 & $\ddagger$ & $\ddagger$ & $\ddagger$ \\
\hline Youth Poll 4 (Nov 02) & $\ddagger$ & 6.9 & 6.9 & 7.1 & $\ddagger$ & $\ddagger$ & $\ddagger$ \\
\hline Youth Poll 5 (June 03) & 7.6 & 7.5 & 7.6 & 7.3 & 7.2 & 7.1 & $\ddagger$ \\
\hline Youth Poll 6 (Nov 03) & 6.8 & 7.4 & 7.1 & 7.0 & 6.5 & 7.5 & $\ddagger$ \\
\hline Youth Poll 7 (May 04) & 6.5 & 7.2 & 6.8 & 7.4 & $\ddagger$ & 7.0 & $\ddagger$ \\
\hline Youth Poll 8 (Nov 04) & 7.4 & 7.2 & 6.8 & 7.1 & 7.3 & $\ddagger$ & $\ddagger$ \\
\hline
\end{tabular}

\section{Females (age 16-21)}

(mean)

High School Grades

\begin{tabular}{lccccccc}
\cline { 2 - 7 } Year & $\begin{array}{c}\text { Mostly } \\
\text { A's }\end{array}$ & $\begin{array}{c}\text { Mostly A's } \\
\text { \& B's }\end{array}$ & $\begin{array}{c}\text { Mostly } \\
\text { B's }\end{array}$ & $\begin{array}{c}\text { Mostly B's } \\
\text { \& C's }\end{array}$ & $\begin{array}{c}\text { Mostly } \\
\text { C's }\end{array}$ & $\begin{array}{c}\text { Mostly C's } \\
\text { \& D's }\end{array}$ & $\begin{array}{c}\text { Mostly D's } \\
\text { and Lower }\end{array}$ \\
\hline Youth Poll 1 (Apr 01) & QNA & QNA & QNA & QNA & QNA & QNA & QNA \\
Youth Poll 2 (Aug 01) & QNA & QNA & QNA & QNA & QNA & QNA & QNA \\
Youth Poll 3 (Nov 01) & 8.0 & 8.5 & 8.3 & 8.6 & $\ddagger$ & $\ddagger$ & $\ddagger$ \\
Youth Poll 4 (Nov 02) & 7.2 & 7.2 & 7.3 & 7.3 & $\ddagger$ & $\ddagger$ & $\ddagger$ \\
Youth Poll 5 (June 03) & 7.5 & 7.8 & 7.6 & 7.7 & $\ddagger$ & $\ddagger$ & $\ddagger$ \\
Youth Poll 6 (Nov 03) & 7.4 & 7.4 & 6.9 & 7.5 & $\ddagger$ & $\ddagger$ & $\ddagger$ \\
Youth Poll 7 (May 04) & 7.4 & 7.1 & 6.9 & 6.9 & $\ddagger$ & $\ddagger$ & $\ddagger$ \\
Youth Poll 8 (Nov 04) & 7.5 & 7.1 & 6.9 & 7.0 & $\ddagger$ & $\ddagger$ & $\ddagger$
\end{tabular}

${ }^{65}$ ‡Reporting standard not met (too few cases); QNA: Question Not Asked

Note: Changes in sample size and stratification were implemented following November 2002 Youth Poll.

Source: Department of Defense Polls, JAMRS, 2001-2004 (Question: FAV2B). 


\section{Navy Favorability}

TABLE 14-1. Youth Navy favorability: $2001-2004^{66}$

\begin{tabular}{lc}
$\begin{array}{l}\text { Male and Female (age 16-21) } \\
\text { (mean) }\end{array}$ & \\
\cline { 2 - 2 } Year & Mean \\
\hline Youth Poll 1 (Apr 01) & QNA \\
Youth Poll 2 (Aug 01) & QNA \\
Youth Poll 3 (Nov 01) & 8.3 \\
Youth Poll 4 (Nov 02) & 7.2 \\
Youth Poll 5 (June 03) & 7.6 \\
Youth Poll 6 (Nov 03) & 7.2 \\
Youth Poll 7 (May 04) & 7.1 \\
Youth Poll 8 (Nov 04) & 7.1 \\
\hline
\end{tabular}

\begin{tabular}{lc}
$\begin{array}{l}\text { Male (age 16-21) } \\
\text { (mean) }\end{array}$ \\
\cline { 2 - 2 } Year
\end{tabular}

\begin{tabular}{lc}
$\begin{array}{l}\text { Female (age 16-21) } \\
\text { (mean) }\end{array}$ & \\
\cline { 2 - 2 } Year & Mean \\
\hline Youth Poll 1 (Apr 01) & QNA \\
Youth Poll 2 (Aug 01) & QNA \\
Youth Poll 3 (Nov 01) & 8.4 \\
Youth Poll 4 (Nov 02) & 7.5 \\
Youth Poll 5 (June 03) & 7.7 \\
Youth Poll 6 (Nov 03) & 7.4 \\
Youth Poll 7 (May 04) & 7.2 \\
Youth Poll 8 (Nov 04) & 7.1 \\
\hline
\end{tabular}

\footnotetext{
${ }^{66}$ ‡Reporting standard not met (too few cases); QNA: Question Not Asked Note: Changes in sample size and stratification were implemented following November 2002 Youth Poll. Source: Department of Defense Polls, JAMRS, 2001-2004 (Question: FAV2E).
} 


\section{Navy Favorability}

TABLE 14-2. Youth Navy favorability, by racelethnicity: $2001-2004^{67}$

\begin{tabular}{lccc} 
Male and Female (age 16-21) & \multicolumn{3}{c}{ Race/ethnicity } \\
\cline { 2 - 4 } (mean) & White & Black & Hispanic \\
\cline { 2 - 4 } Year & QNA & QNA & QNA \\
\hline Youth Poll 1 (Apr 01) & QNA & QNA & QNA \\
Youth Poll 2 (Aug 01) & 8.3 & 8.4 & 8.2 \\
Youth Poll 3 (Nov 01) & 7.3 & 7.0 & 7.1 \\
Youth Poll 4 (Nov 02) & 7.7 & 7.0 & 7.6 \\
Youth Poll 5 (June 03) & 7.3 & 6.9 & 7.3 \\
Youth Poll 6 (Nov 03) & 7.2 & 6.8 & 7.2 \\
Youth Poll 7 (May 04) & 7.3 & 6.3 & 7.1 \\
Youth Poll 8 (Nov 04) & & & \\
\hline
\end{tabular}

\begin{tabular}{lccc} 
Male (age 16-21) & \multicolumn{3}{c}{ Racelethnicity } \\
\cline { 2 - 4 } (mean) & White & Black & Hispanic \\
\cline { 2 - 4 } Year & QNA & QNA & QNA \\
\hline Youth Poll 1 (Apr 01) & QNA & QNA & QNA \\
Youth Poll 2 (Aug 01) & 8.2 & 8.4 & 8.3 \\
Youth Poll 3 (Nov 01) & 7.0 & 7.0 & 6.8 \\
Youth Poll 4 (Nov 02) & 7.5 & 6.9 & 7.4 \\
Youth Poll 5 (June 03) & 7.2 & 6.9 & 7.2 \\
Youth Poll 6 (Nov 03) & 7.2 & 6.9 & 7.0 \\
Youth Poll 7 (May 04) & 7.2 & 6.2 & 7.0 \\
Youth Poll 8 (Nov 04) & & &
\end{tabular}

\begin{tabular}{lccc} 
Female (age 16-21) & \multicolumn{3}{c}{ Racelethnicity } \\
\cline { 2 - 4 } (mean) & White & Black & Hispanic \\
\cline { 2 - 4 } Year & QNA & QNA & QNA \\
\hline Youth Poll 1 (Apr 01) & QNA & QNA & QNA \\
Youth Poll 2 (Aug 01) & 8.5 & 8.5 & 8.1 \\
Youth Poll 3 (Nov 01) & 7.6 & 6.9 & 7.3 \\
Youth Poll 4 (Nov 02) & 7.9 & 7.1 & 7.9 \\
Youth Poll 5 (June 03) & 7.5 & 6.9 & 7.4 \\
Youth Poll 6 (Nov 03) & 7.3 & 6.7 & 7.3 \\
Youth Poll 7 (May 04) & 7.3 & 6.4 & 7.2 \\
Youth Poll 8 (Nov 04) & & & \\
\hline
\end{tabular}

${ }^{67}$ Due to relatively small sample sizes, American Indian, Alaska Native, Asian, and Pacific Islander are included in the total but are not shown separately. Black includes African American, and Hispanic includes Latino. Race categories exclude Hispanic origin unless specified otherwise.

†Reporting standard not met (too few cases); QNA: Question Not Asked

Note: Changes in sample size and stratification were implemented following November 2002 Youth Poll.

Source: Department of Defense Polls, JAMRS, 2001-2004 (Question: FAV2E). 


\section{Navy Favorability}

JAMRS

TABLE 14-3. Youth Navy favorability, by age: $2001-2004^{68}$

\begin{tabular}{|c|c|c|c|c|c|c|}
\hline \multirow{3}{*}{$\begin{array}{l}\text { Male and Female (age 16-21) } \\
\text { (mean) } \\
\text { Year }\end{array}$} & & & & & & \\
\hline & \multicolumn{6}{|c|}{ Age } \\
\hline & 16 & 17 & 18 & 19 & 20 & 21 \\
\hline Youth Poll 1 (Apr 01) & QNA & QNA & QNA & QNA & QNA & QNA \\
\hline Youth Poll 2 (Aug 01) & QNA & QNA & QNA & QNA & QNA & QNA \\
\hline Youth Poll 3 (Nov 01) & 8.4 & 8.2 & 8.3 & 8.6 & 8.4 & 8.3 \\
\hline Youth Poll 4 (Nov 02) & 7.5 & 7.2 & 6.9 & 7.1 & 6.9 & 7.6 \\
\hline Youth Poll 5 (June 03) & 7.7 & 7.6 & 7.4 & 7.5 & 7.6 & 7.7 \\
\hline Youth Poll 6 (Nov 03) & 7.3 & 7.2 & 7.2 & 7.3 & 7.3 & 7.1 \\
\hline Youth Poll 7 (May 04) & 7.4 & 7.2 & 7.2 & 7.4 & 6.8 & 6.9 \\
\hline Youth Poll 8 (Nov 04) & 7.3 & 7.0 & 7.2 & 6.8 & 7.0 & 7.1 \\
\hline
\end{tabular}

\begin{tabular}{lcccccc} 
Male (age 16-21) & \multicolumn{7}{c}{ Age } \\
\cline { 2 - 7 } (mean) & $\mathbf{1 6}$ & $\mathbf{1 7}$ & $\mathbf{1 8}$ & $\mathbf{1 9}$ & $\mathbf{2 0}$ & $\mathbf{2 1}$ \\
\cline { 2 - 7 } Year & QNA & QNA & QNA & QNA & QNA & QNA \\
\hline Youth Poll 1 (Apr 01) & QNA & QNA & QNA & QNA & QNA & QNA \\
Youth Poll 2 (Aug 01) & 8.4 & 8.1 & 8.1 & 8.3 & 8.2 & 8.4 \\
Youth Poll 3 (Nov 01) & 7.5 & 7.0 & 6.9 & 6.4 & 6.6 & 7.3 \\
Youth Poll 4 (Nov 02) & 7.5 & 7.3 & 7.3 & 7.4 & 7.3 & 7.7 \\
Youth Poll 5 (June 03) & 7.1 & 7.1 & 7.1 & 7.2 & 7.2 & 7.0 \\
Youth Poll 6 (Nov 03) & 7.3 & 7.3 & 7.0 & 7.4 & 6.6 & 7.4 \\
Youth Poll 7 (May 04) & 7.3 & 6.8 & 7.4 & 6.7 & 7.0 & 7.1 \\
Youth Poll 8 (Nov 04) & & & & & & 7.0 \\
\hline
\end{tabular}

\section{Female (age 16-21)}

(mean)

Year

Youth Poll 1 (Apr 01)

Youth Poll 2 (Aug 01)

Youth Poll 3 (Nov 01)

Youth Poll 4 (Nov 02)

Youth Poll 5 (June 03)

Youth Poll 6 (Nov 03)

Youth Poll 7 (May 04)

\begin{tabular}{cccccc}
\hline \multicolumn{7}{c}{ Age } \\
\hline $\mathbf{1 6}$ & $\mathbf{1 7}$ & $\mathbf{1 8}$ & $\mathbf{1 9}$ & $\mathbf{2 0}$ & $\mathbf{2 1}$ \\
QNA & QNA & QNA & QNA & QNA & QNA \\
QNA & QNA & QNA & QNA & QNA & QNA \\
8.3 & 8.2 & 8.5 & 8.8 & 8.5 & 8.2 \\
7.6 & 7.4 & 6.9 & 7.7 & 7.2 & 7.7 \\
7.9 & 8.0 & 7.4 & 7.5 & 7.9 & 7.7 \\
7.5 & 7.3 & 7.3 & 7.3 & 7.5 & 7.2 \\
7.5 & 7.1 & 7.4 & 7.5 & 7.1 & 6.5 \\
7.2 & 7.1 & 7.1 & 7.0 & 7.0 & 7.2 \\
\hline
\end{tabular}

Youth Poll 8 (Nov 04)

${ }^{68}$ ‡Reporting standard not met (too few cases); QNA: Question Not Asked

Note: Changes in sample size and stratification were implemented following November 2002 Youth Poll.

Source: Department of Defense Polls, JAMRS, 2001-2004 (Question: FAV2E).

Page A-68 DoD May 2004 Youth Poll 


\section{Navy Favorability}

JAMRS

TABLE 14-4. Youth Navy favorability, by geographic region: $2001-2004^{69}$

\section{Male and Female (age 16-21)}

\begin{tabular}{lccccccccc}
\cline { 2 - 10 } (mean) & \multicolumn{10}{c}{ Geographic Region } \\
\cline { 2 - 11 } Year & $\begin{array}{c}\text { New } \\
\text { England }\end{array}$ & $\begin{array}{c}\text { Mid } \\
\text { Atlantic }\end{array}$ & $\begin{array}{c}\text { East } \\
\text { North } \\
\text { Central }\end{array}$ & $\begin{array}{c}\text { West } \\
\text { North } \\
\text { Central }\end{array}$ & $\begin{array}{c}\text { South } \\
\text { Atlantic }\end{array}$ & $\begin{array}{c}\text { East } \\
\text { South } \\
\text { central }\end{array}$ & $\begin{array}{c}\text { West } \\
\text { South } \\
\text { Central }\end{array}$ & Mountain & Pacific \\
\hline Youth Poll 1 (Apr 01) & QNA & QNA & QNA & QNA & QNA & QNA & QNA & QNA & QNA \\
Youth Poll 2 (Aug 01) & QNA & QNA & QNA & QNA & QNA & QNA & QNA & QNA & QNA \\
Youth Poll 3 (Nov 01) & $\ddagger$ & $\ddagger$ & $\ddagger$ & $\ddagger$ & $\ddagger$ & $\ddagger$ & $\ddagger$ & $\ddagger$ & $\ddagger$ \\
Youth Poll 4 (Nov 02) & $\ddagger$ & $\ddagger$ & $\ddagger$ & $\ddagger$ & $\ddagger$ & $\ddagger$ & $\ddagger$ & $\ddagger$ & $\ddagger$ \\
Youth Poll 5 (June 03) & 7.5 & 7.7 & 7.6 & 7.6 & 7.6 & 7.5 & 7.7 & 7.5 & 7.4 \\
Youth Poll 6 (Nov 03) & 7.3 & 7.2 & 7.1 & 7.4 & 7.2 & 7.6 & 7.3 & 7.2 & 7.2 \\
Youth Poll 7 (May 04) & 7.7 & 7.0 & 7.1 & 7.3 & 7.5 & 7.3 & 7.1 & 7.0 & 6.9 \\
Youth Poll 8 (Nov 04) & 6.7 & 6.8 & 7.1 & 7.3 & 7.1 & 6.7 & 7.1 & 7.6 & 7.1
\end{tabular}

Male (age 16-21)

(mean)

Year

Youth Poll 1 (Apr 01)

Youth Poll 2 (Aug 01)

Youth Poll 3 (Nov 01)

Youth Poll 4 (Nov 02)

Youth Poll 5 (June 03)

Youth Poll 6 (Nov 03)

Youth Poll 7 (May 04)

Youth Poll 8 (Nov 04)

\begin{tabular}{ccccccccc}
\hline \multicolumn{10}{c}{ Geographic Region } \\
\hline $\begin{array}{c}\text { New } \\
\text { England }\end{array}$ & $\begin{array}{c}\text { Mid } \\
\text { Atlantic }\end{array}$ & $\begin{array}{c}\text { East } \\
\text { North } \\
\text { Central }\end{array}$ & $\begin{array}{c}\text { West } \\
\text { North } \\
\text { Central }\end{array}$ & $\begin{array}{c}\text { South } \\
\text { Atlantic }\end{array}$ & $\begin{array}{c}\text { East } \\
\text { South } \\
\text { Central }\end{array}$ & $\begin{array}{c}\text { West } \\
\text { South } \\
\text { Central }\end{array}$ & Mountain & Pacific \\
QNA & QNA & QNA & QNA & QNA & QNA & QNA & QNA & QNA \\
QNA & QNA & QNA & QNA & QNA & QNA & QNA & QNA & QNA \\
$\ddagger$ & $\ddagger$ & $\ddagger$ & $\ddagger$ & $\ddagger$ & $\ddagger$ & $\ddagger$ & $\ddagger$ & $\ddagger$ \\
$\ddagger$ & $\ddagger$ & $\ddagger$ & $\ddagger$ & $\ddagger$ & $\ddagger$ & $\ddagger$ & $\ddagger$ & $\ddagger$ \\
$\ddagger$ & 7.8 & 7.4 & 7.3 & 7.5 & $\ddagger$ & 7.6 & 7.3 & 7.2 \\
$\ddagger$ & 7.1 & 6.8 & 7.2 & 7.0 & $\ddagger$ & 7.4 & $\ddagger$ & 7.0 \\
$\ddagger$ & 6.8 & 7.3 & $\ddagger$ & 7.3 & $\ddagger$ & 6.8 & $\ddagger$ & 7.1 \\
$\ddagger$ & 6.8 & 7.2 & 7.3 & 7.2 & $\ddagger$ & 6.9 & 7.5 & 6.9 \\
\hline
\end{tabular}

\section{Female (age 16-21)}

(mean)

\begin{tabular}{lccccccccc}
\cline { 2 - 8 } Year & $\begin{array}{c}\text { New } \\
\text { England }\end{array}$ & $\begin{array}{c}\text { Mid } \\
\text { Atlantic }\end{array}$ & $\begin{array}{c}\text { East } \\
\text { North } \\
\text { Central }\end{array}$ & $\begin{array}{c}\text { West } \\
\text { North } \\
\text { Central }\end{array}$ & $\begin{array}{c}\text { South } \\
\text { Atlantic }\end{array}$ & $\begin{array}{c}\text { East } \\
\text { South } \\
\text { Central }\end{array}$ & $\begin{array}{c}\text { West } \\
\text { South } \\
\text { Central }\end{array}$ & Mountain & Pacific \\
\hline Youth Poll 1 (Apr 01) & QNA & QNA & QNA & QNA & QNA & QNA & QNA & QNA & QNA \\
Youth Poll 2 (Aug 01) & QNA & QNA & QNA & QNA & QNA & QNA & QNA & QNA & QNA \\
Youth Poll 3 (Nov 01) & $\ddagger$ & $\ddagger$ & $\ddagger$ & $\ddagger$ & $\ddagger$ & $\ddagger$ & $\ddagger$ & $\ddagger$ & $\ddagger$ \\
Youth Poll 4 (Nov 02) & $\ddagger$ & $\ddagger$ & $\ddagger$ & $\ddagger$ & $\ddagger$ & $\ddagger$ & $\ddagger$ & $\ddagger$ & $\ddagger$ \\
Youth Poll 5 (June 03) & $\ddagger$ & 7.5 & 7.8 & 7.9 & 7.7 & $\ddagger$ & 7.8 & 7.7 & 7.7 \\
Youth Poll 6 (Nov 03) & $\ddagger$ & 7.2 & 7.3 & 7.6 & 7.3 & 7.6 & 7.2 & 7.3 & 7.5 \\
Youth Poll 7 (May 04) & $\ddagger$ & 7.3 & 6.9 & $\ddagger$ & 7.6 & $\ddagger$ & 7.4 & $\ddagger$ & 6.7 \\
Youth Poll 8 (Nov 04) & $\ddagger$ & 6.8 & 7.0 & 7.3 & 7.1 & $\ddagger$ & 7.3 & 7.6 & 7.2 \\
\hline
\end{tabular}

${ }^{69} \ddagger$ Reporting standard not met (too few cases); QNA: Question Not Asked

Note: Changes in sample size and stratification were implemented following November 2002 Youth Poll.

Source: Department of Defense Polls, JAMRS, 2001-2004 (Question: FAV2E). 


\section{Navy Favorability}

JAMRS

TABLE 14-5. Youth Navy favorability, by high school grades: $2001-2004^{70}$

\begin{tabular}{|c|c|c|c|c|c|c|c|}
\hline \multicolumn{8}{|c|}{ Male and Female (age 16-21) } \\
\hline \multirow{2}{*}{$\begin{array}{l}\text { (mean) } \\
\text { Year }\end{array}$} & \multicolumn{7}{|c|}{ High School Grades } \\
\hline & $\begin{array}{l}\text { Mostly } \\
\text { A's }\end{array}$ & $\begin{array}{l}\text { Mostly A's } \\
\& \text { B's }\end{array}$ & $\begin{array}{l}\text { Mostly } \\
\text { B's }\end{array}$ & $\begin{array}{l}\text { Mostly B's } \\
\text { \& C's }\end{array}$ & $\begin{array}{l}\text { Mostly } \\
\text { C's }\end{array}$ & $\begin{array}{l}\text { Mostly C's } \\
\text { \& D's }\end{array}$ & $\begin{array}{l}\text { Mostly D's } \\
\text { and Lower }\end{array}$ \\
\hline Youth Poll 1 (Apr 01) & QNA & QNA & QNA & QNA & QNA & QNA & QNA \\
\hline Youth Poll 2 (Aug 01) & QNA & QNA & QNA & QNA & QNA & QNA & QNA \\
\hline Youth Poll 3 (Nov 01) & 8.1 & 8.4 & 8.4 & 8.4 & $\ddagger$ & $\ddagger$ & $\ddagger$ \\
\hline Youth Poll 4 (Nov 02) & 7.4 & 7.3 & 7.3 & 7.0 & 6.8 & $\ddagger$ & $\ddagger$ \\
\hline Youth Poll 5 (June 03) & 7.7 & 7.8 & 7.5 & 7.4 & 7.0 & 7.5 & $\ddagger$ \\
\hline Youth Poll 6 (Nov 03) & 7.3 & 7.3 & 7.1 & 7.3 & 7.0 & 7.1 & $\ddagger$ \\
\hline Youth Poll 7 (May 04) & 7.3 & 7.3 & 7.1 & 7.1 & 6.8 & 6.8 & $\ddagger$ \\
\hline Youth Poll 8 (Nov 04) & 7.5 & 7.1 & 7.0 & 6.9 & 6.9 & 6.7 & $\ddagger$ \\
\hline \multicolumn{8}{|l|}{ Male (age 16-21) } \\
\hline (mean) & \multicolumn{7}{|c|}{ High School Grades } \\
\hline Year & $\begin{array}{l}\text { Mostly } \\
\text { A's }\end{array}$ & $\begin{array}{l}\text { Mostly A's } \\
\text { \& B's }\end{array}$ & $\begin{array}{l}\text { Mostly } \\
\text { B's }\end{array}$ & $\begin{array}{l}\text { Mostly B's } \\
\text { \& C's }\end{array}$ & $\begin{array}{l}\text { Mostly } \\
\text { C's }\end{array}$ & $\begin{array}{l}\text { Mostly C's } \\
\text { \& D's }\end{array}$ & $\begin{array}{l}\text { Mostly D's } \\
\text { and Lower }\end{array}$ \\
\hline Youth Poll 1 (Apr 01) & QNA & QNA & QNA & QNA & QNA & QNA & QNA \\
\hline Youth Poll 2 (Aug 01) & QNA & QNA & QNA & QNA & QNA & QNA & QNA \\
\hline Youth Poll 3 (Nov 01) & 8.2 & 8.3 & 8.3 & 8.2 & $\ddagger$ & $\ddagger$ & $\ddagger$ \\
\hline Youth Poll 4 (Nov 02) & $\ddagger$ & 7.1 & 7.1 & 6.8 & $\ddagger$ & $\ddagger$ & $\ddagger$ \\
\hline Youth Poll 5 (June 03) & 7.7 & 7.7 & 7.6 & 7.3 & 6.9 & 7.0 & $\ddagger$ \\
\hline Youth Poll 6 (Nov 03) & 7.1 & 7.3 & 7.1 & 7.1 & 6.9 & 7.0 & $\ddagger$ \\
\hline Youth Poll 7 (May 04) & 7.2 & 7.3 & 7.0 & 7.2 & $\ddagger$ & 7.0 & $\ddagger$ \\
\hline Youth Poll 8 (Nov 04) & 7.6 & 7.1 & 7.1 & 6.8 & 7.1 & $\ddagger$ & $\ddagger$ \\
\hline
\end{tabular}

\section{Females (age 16-21)}

(mean)

High School Grades

\begin{tabular}{lccccccc}
\cline { 2 - 7 } Year & $\begin{array}{c}\text { Mostly } \\
\text { A's }\end{array}$ & $\begin{array}{c}\text { Mostly A's } \\
\text { \& B's }\end{array}$ & $\begin{array}{c}\text { Mostly } \\
\text { B's }\end{array}$ & $\begin{array}{c}\text { Mostly B's } \\
\text { \& C's }\end{array}$ & $\begin{array}{c}\text { Mostly } \\
\text { C's }\end{array}$ & $\begin{array}{c}\text { Mostly C's } \\
\text { \& D's }\end{array}$ & $\begin{array}{c}\text { Mostly D's } \\
\text { and Lower }\end{array}$ \\
\hline Youth Poll 1 (Apr 01) & QNA & QNA & QNA & QNA & QNA & QNA & QNA \\
Youth Poll 2 (Aug 01) & QNA & QNA & QNA & QNA & QNA & QNA & QNA \\
Youth Poll 3 (Nov 01) & 8.1 & 8.4 & 8.5 & 8.7 & $\ddagger$ & $\ddagger$ & $\ddagger$ \\
Youth Poll 4 (Nov 02) & 7.5 & 7.4 & 7.5 & 7.3 & $\ddagger$ & $\ddagger$ & $\ddagger$ \\
Youth Poll 5 (June 03) & 7.7 & 7.9 & 7.5 & 7.6 & $\ddagger$ & $\ddagger$ & $\ddagger$ \\
Youth Poll 6 (Nov 03) & 7.5 & 7.3 & 7.1 & 7.5 & $\ddagger$ & $\ddagger$ & $\ddagger$ \\
Youth Poll 7 (May 04) & 7.4 & 7.2 & 7.2 & 6.9 & $\ddagger$ & $\ddagger$ & $\ddagger$ \\
Youth Poll 8 (Nov 04) & 7.5 & 7.1 & 7.0 & 7.0 & $\ddagger$ & $\ddagger$ & $\ddagger$
\end{tabular}

${ }^{70} \ddagger$ Reporting standard not met (too few cases); QNA: Question Not Asked Note: Changes in sample size and stratification were implemented following November 2002 Youth Poll. Source: Department of Defense Polls, JAMRS, 2001-2004 (Question: FAV2E). 


\section{Marine Corps Favorability}

TABLE 15-1. Youth Marine Corps favorability: $2001-2004^{71}$

\begin{tabular}{lc}
$\begin{array}{l}\text { Male and Female (age 16-21) } \\
\text { (mean) }\end{array}$ & \\
\cline { 2 - 2 } Year & Mean \\
\hline Youth Poll 1 (Apr 01) & QNA \\
Youth Poll 2 (Aug 01) & QNA \\
Youth Poll 3 (Nov 01) & 8.4 \\
Youth Poll 4 (Nov 02) & 7.2 \\
Youth Poll 5 (June 03) & 7.7 \\
Youth Poll 6 (Nov 03) & 7.4 \\
Youth Poll 7 (May 04) & 7.2 \\
Youth Poll 8 (Nov 04) & 7.2 \\
\hline
\end{tabular}

\begin{tabular}{lc}
$\begin{array}{l}\text { Male (age 16-21) } \\
\text { (mean) }\end{array}$ \\
\cline { 2 - 2 } Year & Mean \\
\hline Youth Poll 1 (Apr 01) & QNA \\
Youth Poll 2 (Aug 01) & QNA \\
Youth Poll 3 (Nov 01) & 8.4 \\
Youth Poll 4 (Nov 02) & 7.2 \\
Youth Poll 5 (June 03) & 7.7 \\
Youth Poll 6 (Nov 03) & 7.4 \\
Youth Poll 7 (May 04) & 7.3 \\
Youth Poll 8 (Nov 04) & 7.3 \\
\hline
\end{tabular}

\begin{tabular}{lc}
$\begin{array}{l}\text { Female (age 16-21) } \\
\text { (mean) }\end{array}$ & \\
\cline { 2 - 2 } Year & Mean \\
\hline Youth Poll 1 (Apr 01) & QNA \\
Youth Poll 2 (Aug 01) & QNA \\
Youth Poll 3 (Nov 01) & 8.4 \\
Youth Poll 4 (Nov 02) & 7.3 \\
Youth Poll 5 (June 03) & 7.8 \\
Youth Poll 6 (Nov 03) & 7.3 \\
Youth Poll 7 (May 04) & 7.2 \\
Youth Poll 8 (Nov 04) & 7.0 \\
\hline
\end{tabular}

${ }^{71}$ ‡Reporting standard not met (too few cases); QNA: Question Not Asked Note: Changes in sample size and stratification were implemented following November 2002 Youth Poll. Source: Department of Defense Polls, JAMRS, 2001-2004 (Question: FAV2D). 


\section{Marine Corps Favorability}

TABLE 15-2. Youth Marine Corps favorability, by race/ethnicity: $2001-2004^{72}$

\begin{tabular}{lccc} 
Male and Female (age 16-21) & \multicolumn{3}{c}{ Racelethnicity } \\
\cline { 2 - 4 } (mean) & White & Black & Hispanic \\
\cline { 2 - 4 } Year & QNA & QNA & QNA \\
\hline Youth Poll 1 (Apr 01) & QNA & QNA & QNA \\
Youth Poll 2 (Aug 01) & 8.5 & 8.3 & 8.3 \\
Youth Poll 3 (Nov 01) & 7.4 & 6.7 & 7.2 \\
Youth Poll 4 (Nov 02) & 7.9 & 6.5 & 8.1 \\
Youth Poll 5 (June 03) & 7.5 & 6.7 & 7.4 \\
Youth Poll 6 (Nov 03) & 7.4 & 6.4 & 7.3 \\
Youth Poll 7 (May 04) & 7.4 & 6.0 & 7.4 \\
Youth Poll 8 (Nov 04) & & & \\
\hline
\end{tabular}

\begin{tabular}{lccc} 
Male (age 16-21) & \multicolumn{3}{c}{ Racelethnicity } \\
\cline { 2 - 4 } (mean) & White & Black & Hispanic \\
\cline { 2 - 4 } Year & QNA & QNA & QNA \\
\hline Youth Poll 1 (Apr 01) & QNA & QNA & QNA \\
Youth Poll 2 (Aug 01) & 8.5 & 8.5 & 8.1 \\
Youth Poll 3 (Nov 01) & 7.3 & 6.8 & 7.3 \\
Youth Poll 4 (Nov 02) & 7.8 & 6.4 & 8.0 \\
Youth Poll 5 (June 03) & 7.5 & 6.8 & 7.4 \\
Youth Poll 6 (Nov 03) & 7.4 & 6.6 & 7.4 \\
Youth Poll 7 (May 04) & 7.4 & 6.1 & 7.5 \\
Youth Poll 8 (Nov 04) & & & \\
\hline
\end{tabular}

\section{Female (age 16-21)}

(mean)

Year

Youth Poll 1 (Apr 01)

Youth Poll 2 (Aug 01)

Youth Poll 3 (Nov 01)

Youth Poll 4 (Nov 02)

Youth Poll 5 (June 03)

Youth Poll 6 (Nov 03)

Youth Poll 7 (May 04)

Youth Poll 8 (Nov 04)

\begin{tabular}{ccc}
\hline \multicolumn{3}{c}{ Racelethnicity } \\
\hline White & Black & Hispanic \\
\hline QNA & QNA & QNA \\
QNA & QNA & QNA \\
8.5 & 8.2 & 8.4 \\
7.4 & 6.5 & 7.2 \\
8.0 & 6.7 & 8.2 \\
7.5 & 6.6 & 7.4 \\
7.4 & 6.2 & 7.3 \\
7.3 & 6.0 & 7.2 \\
\hline
\end{tabular}

\footnotetext{
${ }^{72}$ Due to relatively small sample sizes, American Indian, Alaska Native, Asian, and Pacific Islander are included in the total but are not shown separately. Black includes African American, and Hispanic includes Latino. Race categories exclude Hispanic origin unless specified otherwise.

‡Reporting standard not met (too few cases); QNA: Question Not Asked

Note: Changes in sample size and stratification were implemented following November 2002 Youth Poll.

Source: Department of Defense Polls, JAMRS, 2001-2004 (Question: FAV2D).
} 


\section{Marine Corps Favorability}

JAMRS

TABLE 15-3. Youth Marine Corps favorability, by age: $2001-2004^{73}$

\begin{tabular}{|c|c|c|c|c|c|c|}
\hline \multirow{3}{*}{$\begin{array}{l}\text { Male and Female (age 16-21) } \\
\text { (mean) } \\
\text { Year }\end{array}$} & & & & & & \\
\hline & \multicolumn{6}{|c|}{ Age } \\
\hline & 16 & 17 & 18 & 19 & 20 & 21 \\
\hline Youth Poll 1 (Apr 01) & QNA & QNA & QNA & QNA & QNA & QNA \\
\hline Youth Poll 2 (Aug 01) & QNA & QNA & QNA & QNA & QNA & QNA \\
\hline Youth Poll 3 (Nov 01) & 8.3 & 8.4 & 8.4 & 8.8 & 8.3 & 8.3 \\
\hline Youth Poll 4 (Nov 02) & 7.4 & 7.2 & 7.0 & 7.0 & 7.0 & 7.7 \\
\hline Youth Poll 5 (June 03) & 7.9 & 7.8 & 7.5 & 7.7 & 7.7 & 7.7 \\
\hline Youth Poll 6 (Nov 03) & 7.4 & 7.2 & 7.3 & 7.3 & 7.4 & 7.6 \\
\hline Youth Poll 7 (May 04) & 7.4 & 7.2 & 7.2 & 7.4 & 7.1 & 7.0 \\
\hline Youth Poll 8 (Nov 04) & 7.2 & 7.2 & 7.2 & 7.0 & 7.1 & 7.2 \\
\hline
\end{tabular}

\begin{tabular}{lcccccc} 
Male (age 16-21) & \multicolumn{7}{c}{ Age } \\
\cline { 2 - 7 } (mean) & $\mathbf{1 6}$ & $\mathbf{1 7}$ & $\mathbf{1 8}$ & $\mathbf{1 9}$ & $\mathbf{2 0}$ & $\mathbf{2 1}$ \\
\cline { 2 - 7 } Year & QNA & QNA & QNA & QNA & QNA & QNA \\
\hline Youth Poll 1 (Apr 01) & QNA & QNA & QNA & QNA & QNA & QNA \\
Youth Poll 2 (Aug 01) & 8.4 & 8.5 & 8.3 & 8.7 & 8.3 & 8.4 \\
Youth Poll 3 (Nov 01) & 7.5 & 7.3 & 6.8 & 6.4 & 7.2 & 7.9 \\
Youth Poll 4 (Nov 02) & 7.9 & 7.7 & 7.5 & 7.8 & 7.5 & 7.7 \\
Youth Poll 5 (June 03) & 7.5 & 7.2 & 7.3 & 7.4 & 7.2 & 7.8 \\
Youth Poll 6 (Nov 03) & 7.4 & 7.7 & 7.0 & 7.5 & 6.9 & 7.3 \\
Youth Poll 7 (May 04) & 7.3 & 7.4 & 7.7 & 7.0 & 7.1 & 7.2 \\
Youth Poll 8 (Nov 04) & & & & & & 7.3 \\
\hline
\end{tabular}

\section{Female (age 16-21)}

(mean)

Year

Youth Poll 1 (Apr 01)

Youth Poll 2 (Aug 01)

Youth Poll 3 (Nov 01)

Youth Poll 4 (Nov 02)

Youth Poll 5 (June 03)

Youth Poll 6 (Nov 03)

Youth Poll 7 (May 04)

\begin{tabular}{cccccc}
\hline \multicolumn{7}{c}{ Age } \\
\hline $\mathbf{1 6}$ & $\mathbf{1 7}$ & $\mathbf{1 8}$ & $\mathbf{1 9}$ & $\mathbf{2 0}$ & $\mathbf{2 1}$ \\
QNA & QNA & QNA & QNA & QNA & QNA \\
QNA & QNA & QNA & QNA & QNA & QNA \\
8.2 & 8.3 & 8.6 & 8.9 & 8.2 & 8.3 \\
7.3 & 7.1 & 7.1 & 7.5 & 6.9 & 7.6 \\
7.9 & 7.8 & 7.6 & 7.6 & 7.9 & 7.7 \\
7.3 & 7.2 & 7.3 & 7.1 & 7.6 & 7.3 \\
7.5 & 6.9 & 7.4 & 7.4 & 7.3 & 6.8 \\
7.1 & 7.0 & 6.7 & 7.0 & 7.1 & 7.2 \\
\hline
\end{tabular}

Youth Poll 8 (Nov 04)

${ }^{73}$ ‡Reporting standard not met (too few cases); QNA: Question Not Asked

Note: Changes in sample size and stratification were implemented following November 2002 Youth Poll.

Source: Department of Defense Polls, JAMRS, 2001-2004 (Question: FAV2D).

Page A-73 DoD May 2004 Youth Poll 


\section{Marine Corps Favorability}

JAMRS

TABLE 15-4. Youth Marine Corps favorability, by geographic region: $2001-2004^{74}$

\section{Male and Female (age 16-21)}

(mean)

Year

Youth Poll 1 (Apr 01)

Youth Poll 2 (Aug 01)

Youth Poll 3 (Nov 01)

Youth Poll 4 (Nov 02)

\begin{tabular}{ccccccccc}
\hline \multicolumn{10}{c}{ Geographic Region } \\
\hline $\begin{array}{c}\text { New } \\
\text { England }\end{array}$ & $\begin{array}{c}\text { Mid } \\
\text { Atlantic }\end{array}$ & $\begin{array}{c}\text { East } \\
\text { North } \\
\text { Central }\end{array}$ & $\begin{array}{c}\text { West } \\
\text { North } \\
\text { Central }\end{array}$ & $\begin{array}{c}\text { South } \\
\text { Atlantic }\end{array}$ & $\begin{array}{c}\text { East } \\
\text { South } \\
\text { Central }\end{array}$ & $\begin{array}{c}\text { West } \\
\text { South } \\
\text { Central }\end{array}$ & Mountain & Pacific \\
QNA & QNA & QNA & QNA & QNA & QNA & QNA & QNA & QNA \\
QNA & QNA & QNA & QNA & QNA & QNA & QNA & QNA & QNA \\
$\ddagger$ & $\ddagger$ & $\ddagger$ & $\ddagger$ & $\ddagger$ & $\ddagger$ & $\ddagger$ & $\ddagger$ & $\ddagger$ \\
$\ddagger$ & $\ddagger$ & $\ddagger$ & $\ddagger$ & $\ddagger$ & $\ddagger$ & $\ddagger$ & $\ddagger$ & $\ddagger$ \\
7.7 & 7.6 & 7.8 & 7.8 & 7.6 & 7.1 & 7.9 & 7.8 & 7.8 \\
7.3 & 7.3 & 7.3 & 7.6 & 7.1 & 7.8 & 7.4 & 7.7 & 7.3 \\
7.9 & 7.0 & 7.1 & 7.6 & 7.5 & 7.4 & 7.2 & 7.2 & 7.0 \\
6.7 & 6.6 & 7.0 & 7.5 & 7.3 & 7.0 & 7.5 & 7.6 & 7.2 \\
\hline
\end{tabular}

Youth Poll 5 (June 03)

Youth Poll 6 (Nov 03)

Youth Poll 7 (May 04)

6.7

6.6

\begin{tabular}{ccccccccc}
\hline \multicolumn{10}{c}{ Geographic Region } \\
$\begin{array}{c}\text { New } \\
\text { England }\end{array}$ & $\begin{array}{c}\text { Mid } \\
\text { Atlantic }\end{array}$ & $\begin{array}{c}\text { East } \\
\text { North } \\
\text { Central }\end{array}$ & $\begin{array}{c}\text { West } \\
\text { North } \\
\text { Central }\end{array}$ & $\begin{array}{c}\text { South } \\
\text { Atlantic }\end{array}$ & $\begin{array}{c}\text { East } \\
\text { South } \\
\text { Central }\end{array}$ & $\begin{array}{c}\text { West } \\
\text { South } \\
\text { Central }\end{array}$ & Mountain & Pacific \\
QNA & QNA & QNA & QNA & QNA & QNA & QNA & QNA & QNA \\
QNA & QNA & QNA & QNA & QNA & QNA & QNA & QNA & QNA \\
$\ddagger$ & $\ddagger$ & $\ddagger$ & $\ddagger$ & $\ddagger$ & $\ddagger$ & $\ddagger$ & $\ddagger$ & $\ddagger$ \\
$\ddagger$ & $\ddagger$ & $\ddagger$ & $\ddagger$ & $\ddagger$ & $\ddagger$ & $\ddagger$ & $\ddagger$ & $\ddagger$ \\
$\ddagger$ & 7.7 & 7.9 & 7.6 & 7.4 & $\ddagger$ & 7.8 & 7.7 & 7.8 \\
$\ddagger$ & 7.4 & 7.2 & 7.5 & 7.1 & $\ddagger$ & 7.5 & $\ddagger$ & 7.3 \\
$\ddagger$ & 6.9 & 7.3 & $\ddagger$ & 7.5 & $\ddagger$ & 7.1 & $\ddagger$ & 7.0 \\
$\ddagger$ & 6.7 & 7.2 & 7.9 & 7.4 & $\ddagger$ & 7.7 & 7.7 & 7.1 \\
\hline
\end{tabular}

Male (age 16-21)

(mean)

Year

Youth Poll 1 (Apr 01)

Youth Poll 2 (Aug 01)

Youth Poll 3 (Nov 01)

Youth Poll 4 (Nov 02)

Youth Poll 5 (June 03)

Youth Poll 6 (Nov 03)

Youth Poll 7 (May 04)

\section{Geographic Region}

Youth Poll 8 (Nov 04)

Female (age 16-21)

(mean)

Year

Youth Poll 1 (Apr 01)

Youth Poll 2 (Aug 01)

Youth Poll 3 (Nov 01)

Youth Poll 4 (Nov 02)

Youth Poll 5 (June 03)

Youth Poll 6 (Nov 03)

Youth Poll 7 (May 04)

Youth Poll 8 (Nov 04)

\begin{tabular}{ccccccccc}
\hline \multicolumn{10}{c}{ Geographic Region } \\
$\begin{array}{c}\text { New } \\
\text { England }\end{array}$ & $\begin{array}{c}\text { Mid } \\
\text { Atlantic }\end{array}$ & $\begin{array}{c}\text { East } \\
\text { North } \\
\text { Central }\end{array}$ & $\begin{array}{c}\text { West } \\
\text { North } \\
\text { Central }\end{array}$ & $\begin{array}{c}\text { South } \\
\text { Atlantic }\end{array}$ & $\begin{array}{c}\text { East } \\
\text { South } \\
\text { Central }\end{array}$ & $\begin{array}{c}\text { West } \\
\text { South } \\
\text { Central }\end{array}$ & Mountain & Pacific \\
QNA & QNA & QNA & QNA & QNA & QNA & QNA & QNA & QNA \\
QNA & QNA & QNA & QNA & QNA & QNA & QNA & QNA & QNA \\
$\ddagger$ & $\ddagger$ & $\ddagger$ & $\ddagger$ & $\ddagger$ & $\ddagger$ & $\ddagger$ & $\ddagger$ & $\ddagger$ \\
$\ddagger$ & $\ddagger$ & $\ddagger$ & $\ddagger$ & $\ddagger$ & $\ddagger$ & $\ddagger$ & $\ddagger$ & $\ddagger$ \\
$\ddagger$ & 7.6 & 7.7 & 8.0 & 7.8 & $\ddagger$ & 8.0 & 7.9 & 7.7 \\
$\ddagger$ & 7.2 & 7.4 & 7.6 & 7.1 & 7.7 & 7.2 & 7.5 & 7.3 \\
$\ddagger$ & 7.2 & 6.9 & $\ddagger$ & 7.5 & $\ddagger$ & 7.3 & $\ddagger$ & 6.9 \\
$\ddagger$ & 6.4 & 6.8 & 6.9 & 7.2 & $\ddagger$ & 7.3 & 7.6 & 7.3 \\
\hline
\end{tabular}

\footnotetext{
${ }^{74}$ ‡Reporting standard not met (too few cases); QNA: Question Not Asked

Note: Changes in sample size and stratification were implemented following November 2002 Youth Poll.

Source: Department of Defense Polls, JAMRS, 2001-2004 (Question: FAV2D).
} 


\section{Marine Corps Favorability}

JAMRS

TABLE 15-5. Youth Marine Corps favorability, by high school grades: $2001-2004^{75}$

\begin{tabular}{|c|c|c|c|c|c|c|c|}
\hline \multicolumn{8}{|c|}{ Male and Female (age 16-21) } \\
\hline \multirow{2}{*}{$\begin{array}{l}\text { (mean) } \\
\text { Year }\end{array}$} & \multicolumn{7}{|c|}{ High School Grades } \\
\hline & $\begin{array}{l}\text { Mostly } \\
\text { A's }\end{array}$ & $\begin{array}{l}\text { Mostly A's } \\
\text { \& B's }\end{array}$ & $\begin{array}{l}\text { Mostly } \\
\text { B's }\end{array}$ & $\begin{array}{l}\text { Mostly B's } \\
\text { \& C's }\end{array}$ & $\begin{array}{l}\text { Mostly } \\
\text { C's }\end{array}$ & $\begin{array}{l}\text { Mostly C's } \\
\text { \& D's }\end{array}$ & $\begin{array}{l}\text { Mostly D's } \\
\text { and Lower }\end{array}$ \\
\hline Youth Poll 1 (Apr 01) & QNA & QNA & QNA & QNA & QNA & QNA & QNA \\
\hline Youth Poll 2 (Aug 01) & QNA & QNA & QNA & QNA & QNA & QNA & QNA \\
\hline Youth Poll 3 (Nov 01) & 8.2 & 8.4 & 8.5 & 8.6 & $\ddagger$ & $\ddagger$ & $\ddagger$ \\
\hline Youth Poll 4 (Nov 02) & 7.3 & 7.2 & 7.4 & 7.1 & 7.0 & $\ddagger$ & $\ddagger$ \\
\hline Youth Poll 5 (June 03) & 7.8 & 7.8 & 7.8 & 7.5 & 7.4 & 7.9 & $\ddagger$ \\
\hline Youth Poll 6 (Nov 03) & 7.5 & 7.4 & 7.2 & 7.4 & 6.7 & 7.6 & $\ddagger$ \\
\hline Youth Poll 7 (May 04) & 7.6 & 7.3 & 7.1 & 7.2 & 6.6 & 7.2 & $\ddagger$ \\
\hline Youth Poll 8 (Nov 04) & 7.6 & 7.2 & 7.0 & 7.0 & 7.1 & 6.9 & $\ddagger$ \\
\hline \multicolumn{8}{|l|}{ Male (age $16-21$ ) } \\
\hline (mean) & \multicolumn{7}{|c|}{ High School Grades } \\
\hline Year & $\begin{array}{c}\text { Mostly } \\
\text { A's }\end{array}$ & $\begin{array}{c}\text { Mostly A's } \\
\text { \& B's }\end{array}$ & $\begin{array}{l}\text { Mostly } \\
\text { B's }\end{array}$ & $\begin{array}{l}\text { Mostly B's } \\
\text { \& C's }\end{array}$ & $\begin{array}{l}\text { Mostly } \\
\text { C's }\end{array}$ & $\begin{array}{l}\text { Mostly C's } \\
\text { \& D's }\end{array}$ & $\begin{array}{l}\text { Mostly D's } \\
\text { and Lower }\end{array}$ \\
\hline Youth Poll 1 (Apr 01) & QNA & QNA & QNA & QNA & QNA & QNA & QNA \\
\hline Youth Poll 2 (Aug 01) & QNA & QNA & QNA & QNA & QNA & QNA & QNA \\
\hline Youth Poll 3 (Nov 01) & 8.3 & 8.4 & 8.6 & 8.6 & $\ddagger$ & $\ddagger$ & $\ddagger$ \\
\hline Youth Poll 4 (Nov 02) & $\ddagger$ & 7.1 & 7.4 & 7.1 & $\ddagger$ & $\ddagger$ & $\ddagger$ \\
\hline Youth Poll 5 (June 03) & 8.0 & 7.8 & 8.0 & 7.5 & 7.4 & 7.5 & $\ddagger$ \\
\hline Youth Poll 6 (Nov 03) & 7.4 & 7.4 & 7.6 & 7.5 & 6.7 & 7.7 & $\ddagger$ \\
\hline Youth Poll 7 (May 04) & 7.4 & 7.4 & 6.9 & 7.4 & $\ddagger$ & 7.5 & $\ddagger$ \\
\hline Youth Poll 8 (Nov 04) & 7.8 & 7.2 & 7.2 & 7.2 & 7.4 & $\ddagger$ & $\ddagger$ \\
\hline
\end{tabular}

\section{Females (age 16-21)}

(mean)

High School Grades

\begin{tabular}{lccccccc}
\cline { 2 - 7 } Year & $\begin{array}{c}\text { Mostly } \\
\text { A's }\end{array}$ & $\begin{array}{c}\text { Mostly A's } \\
\text { \& B's }\end{array}$ & $\begin{array}{c}\text { Mostly } \\
\text { B's }\end{array}$ & $\begin{array}{c}\text { Mostly B's } \\
\text { \& C's }\end{array}$ & $\begin{array}{c}\text { Mostly } \\
\text { C's }\end{array}$ & $\begin{array}{c}\text { Mostly C's } \\
\text { \& D's }\end{array}$ & $\begin{array}{c}\text { Mostly D's } \\
\text { and Lower }\end{array}$ \\
\hline Youth Poll 1 (Apr 01) & QNA & QNA & QNA & QNA & QNA & QNA & QNA \\
Youth Poll 2 (Aug 01) & QNA & QNA & QNA & QNA & QNA & QNA & QNA \\
Youth Poll 3 (Nov 01) & 8.1 & 8.5 & 8.3 & 8.6 & $\ddagger$ & $\ddagger$ & $\ddagger$ \\
Youth Poll 4 (Nov 02) & 7.3 & 7.3 & 7.3 & 7.2 & $\ddagger$ & $\ddagger$ & $\ddagger$ \\
Youth Poll 5 (June 03) & 7.7 & 7.9 & 7.6 & 7.5 & $\ddagger$ & $\ddagger$ & $\ddagger$ \\
Youth Poll 6 (Nov 03) & 7.6 & 7.5 & 6.7 & 7.3 & $\ddagger$ & $\ddagger$ & $\ddagger$ \\
Youth Poll 7 (May 04) & 7.7 & 7.2 & 7.2 & 6.9 & $\ddagger$ & $\ddagger$ & $\ddagger$ \\
Youth Poll 8 (Nov 04) & 7.4 & 7.2 & 6.9 & 6.8 & $\ddagger$ & $\ddagger$ & $\ddagger$ \\
\hline
\end{tabular}

${ }^{75}$ ‡Reporting standard not met (too few cases); QNA: Question Not Asked

Note: Changes in sample size and stratification were implemented following November 2002 Youth Poll.

Source: Department of Defense Polls, JAMRS, 2001-2004 (Question: FAV2D). 


\section{Air Force Favorability}

TABLE 16-1. Youth Air Force favorability: $2001-2004^{76}$

\begin{tabular}{lc}
$\begin{array}{l}\text { Male and Female (age 16-21) } \\
\text { (mean) }\end{array}$ & \\
\cline { 2 - 2 } Year & Mean \\
\hline Youth Poll 1 (Apr 01) & QNA \\
Youth Poll 2 (Aug 01) & QNA \\
Youth Poll 3 (Nov 01) & 8.6 \\
Youth Poll 4 (Nov 02) & 7.6 \\
Youth Poll 5 (June 03) & 7.8 \\
Youth Poll 6 (Nov 03) & 7.5 \\
Youth Poll 7 (May 04) & 7.5 \\
Youth Poll 8 (Nov 04) & 7.4 \\
\hline
\end{tabular}

\begin{tabular}{lc}
$\begin{array}{l}\text { Male (age 16-21) } \\
\text { (mean) }\end{array}$ \\
\cline { 2 - 2 } Year
\end{tabular}

\begin{tabular}{lc}
$\begin{array}{l}\text { Female (age 16-21) } \\
\text { (mean) }\end{array}$ & \\
\cline { 2 - 2 } Year & Mean \\
\hline Youth Poll 1 (Apr 01) & QNA \\
Youth Poll 2 (Aug 01) & QNA \\
Youth Poll 3 (Nov 01) & 8.6 \\
Youth Poll 4 (Nov 02) & 7.8 \\
Youth Poll 5 (June 03) & 7.9 \\
Youth Poll 6 (Nov 03) & 7.6 \\
Youth Poll 7 (May 04) & 7.5 \\
Youth Poll 8 (Nov 04) & 7.4 \\
\hline
\end{tabular}

${ }^{76}$ ‡Reporting standard not met (too few cases); QNA: Question Not Asked Note: Changes in sample size and stratification were implemented following November 2002 Youth Poll. Source: Department of Defense Polls, JAMRS, 2001-2004 (Question: FAV2A). 


\section{Air Force Favorability}

TABLE 16-2. Youth Air Force favorability, by race/ethnicity: $2001-2004^{77}$

\begin{tabular}{lccc} 
Male and Female (age 16-21) & \multicolumn{3}{c}{ Racelethnicity } \\
\cline { 2 - 4 } (mean) & White & Black & Hispanic \\
\cline { 2 - 4 } Year & QNA & QNA & QNA \\
\hline Youth Poll 1 (Apr 01) & QNA & QNA & QNA \\
Youth Poll 2 (Aug 01) & 8.6 & 8.6 & 8.5 \\
Youth Poll 3 (Nov 01) & 7.7 & 7.3 & 7.7 \\
Youth Poll 4 (Nov 02) & 8.0 & 7.3 & 7.9 \\
Youth Poll 5 (June 03) & 7.6 & 7.2 & 7.5 \\
Youth Poll 6 (Nov 03) & 7.6 & 7.0 & 7.6 \\
Youth Poll 7 (May 04) & 7.6 & 6.6 & 7.5 \\
Youth Poll 8 (Nov 04) & & & \\
\hline
\end{tabular}

\begin{tabular}{lccc} 
Male (age 16-21) & \multicolumn{3}{c}{ Racelethnicity } \\
\cline { 2 - 4 } (mean) & White & Black & Hispanic \\
\cline { 2 - 4 } Year & QNA & QNA & QNA \\
\hline Youth Poll 1 (Apr 01) & QNA & QNA & QNA \\
Youth Poll 2 (Aug 01) & 8.6 & 8.6 & 8.6 \\
Youth Poll 3 (Nov 01) & 7.5 & 7.1 & 7.7 \\
Youth Poll 4 (Nov 02) & 7.8 & 7.4 & 7.8 \\
Youth Poll 5 (June 03) & 7.5 & 7.4 & 7.5 \\
Youth Poll 6 (Nov 03) & 7.7 & 7.1 & 7.6 \\
Youth Poll 7 (May 04) & 7.6 & 6.7 & 7.4 \\
Youth Poll 8 (Nov 04) & & &
\end{tabular}

\begin{tabular}{lccc} 
Female (age 16-21) & \multicolumn{3}{c}{ Racelethnicity } \\
\cline { 2 - 4 } (mean) & White & Black & Hispanic \\
\cline { 2 - 4 } Year & QNA & QNA & QNA \\
\hline Youth Poll 1 (Apr 01) & QNA & QNA & QNA \\
Youth Poll 2 (Aug 01) & 8.6 & 8.5 & 8.4 \\
Youth Poll 3 (Nov 01) & 7.8 & 7.5 & 7.7 \\
Youth Poll 4 (Nov 02) & 8.1 & 7.2 & 8.0 \\
Youth Poll 5 (June 03) & 7.7 & 7.0 & 7.6 \\
Youth Poll 6 (Nov 03) & 7.6 & 6.9 & 7.6 \\
Youth Poll 7 (May 04) & 7.6 & 6.6 & 7.7 \\
Youth Poll 8 (Nov 04) & & & \\
\hline
\end{tabular}

\footnotetext{
${ }^{77}$ Due to relatively small sample sizes, American Indian, Alaska Native, Asian, and Pacific Islander are included in the total but are not shown separately. Black includes African American, and Hispanic includes Latino. Race categories exclude Hispanic origin unless specified otherwise.

†Reporting standard not met (too few cases); QNA: Question Not Asked

Note: Changes in sample size and stratification were implemented following November 2002 Youth Poll.

Source: Department of Defense Polls, JAMRS, 2001-2004 (Question: FAV2A).
} 


\section{Air Force Favorability}

JAMRS

TABLE 16-3. Youth Air Force favorability, by age: $2001-2004^{78}$

\begin{tabular}{lcccccc} 
Male and Female (age 16-21) & \multicolumn{7}{c}{ Age } \\
\cline { 2 - 7 } (mean) & 16 & $\mathbf{1 7}$ & $\mathbf{1 8}$ & $\mathbf{1 9}$ & $\mathbf{2 0}$ & $\mathbf{2 1}$ \\
\cline { 2 - 7 } Year & QNA & QNA & QNA & QNA & QNA & QNA \\
\cline { 2 - 7 } Youth Poll 1 (Apr 01) & QNA & QNA & QNA & QNA & QNA & QNA \\
Youth Poll 2 (Aug 01) & 8.6 & 8.5 & 8.6 & 8.6 & 8.6 & 8.5 \\
Youth Poll 3 (Nov 01) & 7.8 & 7.5 & 7.4 & 7.5 & 7.3 & 8.1 \\
Youth Poll 4 (Nov 02) & 8.0 & 7.9 & 7.7 & 7.9 & 7.7 & 7.8 \\
Youth Poll 5 (June 03) & 7.5 & 7.5 & 7.6 & 7.6 & 7.6 & 7.5 \\
Youth Poll 6 (Nov 03) & 7.7 & 7.6 & 7.5 & 7.6 & 7.4 & 7.4 \\
Youth Poll 7 (May 04) & 7.4 & 7.4 & 7.7 & 7.3 & 7.6 & 7.2 \\
Youth Poll 8 (Nov 04) & & & & & & 7.3 \\
\hline
\end{tabular}

\begin{tabular}{lcccccc} 
Male (age 16-21) & \multicolumn{7}{c}{ Age } \\
\cline { 2 - 7 } (mean) & $\mathbf{1 6}$ & $\mathbf{1 7}$ & $\mathbf{1 8}$ & $\mathbf{1 9}$ & $\mathbf{2 0}$ & $\mathbf{2 1}$ \\
\cline { 2 - 7 } Year & QNA & QNA & QNA & QNA & QNA & QNA \\
\hline Youth Poll 1 (Apr 01) & QNA & QNA & QNA & QNA & QNA & QNA \\
Youth Poll 2 (Aug 01) & 8.7 & 8.6 & 8.5 & 8.6 & 8.5 & 8.5 \\
Youth Poll 3 (Nov 01) & 7.8 & 7.5 & 7.3 & 7.2 & 7.1 & 7.8 \\
Youth Poll 4 (Nov 02) & 7.9 & 7.8 & 7.8 & 7.9 & 7.3 & 8.0 \\
Youth Poll 5 (June 03) & 7.5 & 7.5 & 7.4 & 7.6 & 7.4 & 7.5 \\
Youth Poll 6 (Nov 03) & 7.7 & 7.9 & 7.4 & 7.7 & 7.3 & 7.5 \\
Youth Poll 7 (May 04) & 7.5 & 7.4 & 7.8 & 7.4 & 7.7 & 6.8 \\
Youth Poll 8 (Nov 04) & & & & & & \\
\hline
\end{tabular}

\section{Female (age 16-21)}

(mean)

Year

Youth Poll 1 (Apr 01)

Youth Poll 2 (Aug 01)

Youth Poll 3 (Nov 01)

Youth Poll 4 (Nov 02)

Youth Poll 5 (June 03)

Youth Poll 6 (Nov 03)

Youth Poll 7 (May 04)

\begin{tabular}{cccccc}
\hline \multicolumn{7}{c}{ Age } \\
\hline $\mathbf{1 6}$ & $\mathbf{1 7}$ & $\mathbf{1 8}$ & $\mathbf{1 9}$ & $\mathbf{2 0}$ & $\mathbf{2 1}$ \\
QNA & QNA & QNA & QNA & QNA & QNA \\
QNA & QNA & QNA & QNA & QNA & QNA \\
8.5 & 8.4 & 8.7 & 8.7 & 8.6 & 8.6 \\
7.8 & 7.6 & 7.6 & 7.9 & 7.5 & 8.3 \\
8.2 & 8.0 & 7.6 & 7.9 & 8.0 & 7.6 \\
7.5 & 7.4 & 7.7 & 7.6 & 7.8 & 7.5 \\
7.7 & 7.3 & 7.6 & 7.6 & 7.5 & 7.2 \\
7.3 & 7.5 & 7.5 & 7.2 & 7.5 & 7.7 \\
\hline
\end{tabular}

Youth Poll 8 (Nov 04)

${ }^{78}$ fReporting standard not met (too few cases); QNA: Question Not Asked

Note: Changes in sample size and stratification were implemented following November 2002 Youth Poll.

Source: Department of Defense Polls, JAMRS, 2001-2004 (Question: FAV2A).

Page A-78 DoD May 2004 Youth Poll 


\section{Air Force Favorability}

JAMRS

TABLE 16-4. Youth Air Force favorability, by geographic region: $2001-2004^{79}$

\section{Male and Female (age 16-21)}

\begin{tabular}{lccccccccc}
\cline { 2 - 10 } (mean) & \multicolumn{10}{c}{ Geographic Region } \\
\cline { 2 - 11 } Year & $\begin{array}{c}\text { New } \\
\text { England }\end{array}$ & $\begin{array}{c}\text { Mid } \\
\text { Atlantic }\end{array}$ & $\begin{array}{c}\text { East } \\
\text { North } \\
\text { Central }\end{array}$ & $\begin{array}{c}\text { West } \\
\text { North } \\
\text { Central }\end{array}$ & $\begin{array}{c}\text { South } \\
\text { Atlantic }\end{array}$ & $\begin{array}{c}\text { East } \\
\text { South } \\
\text { central }\end{array}$ & $\begin{array}{c}\text { West } \\
\text { South } \\
\text { Central }\end{array}$ & Mountain & Pacific \\
\hline Youth Poll 1 (Apr 01) & QNA & QNA & QNA & QNA & QNA & QNA & QNA & QNA & QNA \\
Youth Poll 2 (Aug 01) & QNA & QNA & QNA & QNA & QNA & QNA & QNA & QNA & QNA \\
Youth Poll 3 (Nov 01) & $\ddagger$ & $\ddagger$ & $\ddagger$ & $\ddagger$ & $\ddagger$ & $\ddagger$ & $\ddagger$ & $\ddagger$ & $\ddagger$ \\
Youth Poll 4 (Nov 02) & $\ddagger$ & $\ddagger$ & $\ddagger$ & $\ddagger$ & $\ddagger$ & $\ddagger$ & $\ddagger$ & $\ddagger$ & $\ddagger$ \\
Youth Poll 5 (June 03) & 7.4 & 7.8 & 7.9 & 7.9 & 7.9 & 7.9 & 8.0 & 7.7 & 7.7 \\
Youth Poll 6 (Nov 03) & 7.4 & 7.6 & 7.4 & 7.5 & 7.5 & 8.0 & 7.6 & 7.4 & 7.5 \\
Youth Poll 7 (May 04) & 7.9 & 7.5 & 7.4 & 7.4 & 7.8 & 7.7 & 7.6 & 7.4 & 7.4 \\
Youth Poll 8 (Nov 04) & 7.1 & 6.9 & 7.2 & 7.8 & 7.6 & 7.7 & 7.7 & 7.7 & 7.4
\end{tabular}

Male (age 16-21)

(mean)

Year

Youth Poll 1 (Apr 01)

Youth Poll 2 (Aug 01)

Youth Poll 3 (Nov 01)

Youth Poll 4 (Nov 02)

Youth Poll 5 (June 03)

Youth Poll 6 (Nov 03)

Youth Poll 7 (May 04)

Youth Poll 8 (Nov 04)

\begin{tabular}{ccccccccc}
\hline \multicolumn{10}{c}{ Geographic Region } \\
\hline $\begin{array}{c}\text { New } \\
\text { England }\end{array}$ & $\begin{array}{c}\text { Mid } \\
\text { Atlantic }\end{array}$ & $\begin{array}{c}\text { East } \\
\text { North } \\
\text { Central }\end{array}$ & $\begin{array}{c}\text { West } \\
\text { North } \\
\text { Central }\end{array}$ & $\begin{array}{c}\text { South } \\
\text { Atlantic }\end{array}$ & $\begin{array}{c}\text { East } \\
\text { South } \\
\text { Central }\end{array}$ & $\begin{array}{c}\text { West } \\
\text { South } \\
\text { Central }\end{array}$ & Mountain & Pacific \\
QNA & QNA & QNA & QNA & QNA & QNA & QNA & QNA & QNA \\
QNA & QNA & QNA & QNA & QNA & QNA & QNA & QNA & QNA \\
$\ddagger$ & $\ddagger$ & $\ddagger$ & $\ddagger$ & $\ddagger$ & $\ddagger$ & $\ddagger$ & $\ddagger$ & $\ddagger$ \\
$\ddagger$ & $\ddagger$ & $\ddagger$ & $\ddagger$ & $\ddagger$ & $\ddagger$ & $\ddagger$ & $\ddagger$ & $\ddagger$ \\
$\ddagger$ & 7.9 & 7.9 & 7.5 & 7.9 & $\ddagger$ & 8.0 & 7.5 & 7.7 \\
$\ddagger$ & 7.9 & 7.2 & 7.1 & 7.5 & $\ddagger$ & 7.6 & $\ddagger$ & 7.5 \\
$\ddagger$ & 7.5 & 7.6 & $\ddagger$ & 7.8 & $\ddagger$ & 7.5 & $\ddagger$ & 7.4 \\
$\ddagger$ & 6.8 & 7.4 & 7.9 & 7.7 & $\ddagger$ & 7.7 & 7.7 & 7.3 \\
\hline
\end{tabular}

\section{Female (age 16-21)}

(mean)

\section{Geographic Region}

\begin{tabular}{lccccccccc}
\cline { 2 - 8 } Year & $\begin{array}{c}\text { New } \\
\text { England }\end{array}$ & $\begin{array}{c}\text { Mid } \\
\text { Atlantic }\end{array}$ & $\begin{array}{c}\text { East } \\
\text { North } \\
\text { Central }\end{array}$ & $\begin{array}{c}\text { West } \\
\text { North } \\
\text { Central }\end{array}$ & $\begin{array}{c}\text { South } \\
\text { Atlantic }\end{array}$ & $\begin{array}{c}\text { East } \\
\text { South } \\
\text { Central }\end{array}$ & $\begin{array}{c}\text { West } \\
\text { South } \\
\text { Central }\end{array}$ & Mountain & Pacific \\
\hline Youth Poll 1 (Apr 01) & QNA & QNA & QNA & QNA & QNA & QNA & QNA & QNA & QNA \\
Youth Poll 2 (Aug 01) & QNA & QNA & QNA & QNA & QNA & QNA & QNA & QNA & QNA \\
Youth Poll 3 (Nov 01) & $\ddagger$ & $\ddagger$ & $\ddagger$ & $\ddagger$ & $\ddagger$ & $\ddagger$ & $\ddagger$ & $\ddagger$ & $\ddagger$ \\
Youth Poll 4 (Nov 02) & $\ddagger$ & $\ddagger$ & $\ddagger$ & $\ddagger$ & $\ddagger$ & $\ddagger$ & $\ddagger$ & $\ddagger$ & $\ddagger$ \\
Youth Poll 5 (June 03) & $\ddagger$ & 7.7 & 7.9 & 8.2 & 7.8 & $\ddagger$ & 8.0 & 8.0 & 7.9 \\
Youth Poll 6 (Nov 03) & $\ddagger$ & 7.3 & 7.6 & 8.0 & 7.5 & 7.8 & 7.7 & 7.7 & 7.6 \\
Youth Poll 7 (May 04) & $\ddagger$ & 7.5 & 7.1 & $\ddagger$ & 7.8 & $\ddagger$ & 7.6 & $\ddagger$ & 7.4 \\
Youth Poll 8 (Nov 04) & $\ddagger$ & 7.0 & 7.1 & 7.5 & 7.6 & $\ddagger$ & 7.8 & 7.8 & 7.6 \\
\hline
\end{tabular}

\footnotetext{
${ }^{79}$ ‡Reporting standard not met (too few cases); QNA: Question Not Asked Note: Changes in sample size and stratification were implemented following November 2002 Youth Poll. Source: Department of Defense Polls, JAMRS, 2001-2004 (Question: FAV2A).
} 


\section{Air Force Favorability}

JAMRS

TABLE 16-5. Youth Air Force favorability, by high school grades: $2001-2004^{80}$

\begin{tabular}{|c|c|c|c|c|c|c|c|}
\hline \multicolumn{8}{|c|}{ Male and Female (age 16-21) } \\
\hline \multirow{2}{*}{$\begin{array}{l}\text { (mean) } \\
\text { Year }\end{array}$} & \multicolumn{7}{|c|}{ High School Grades } \\
\hline & $\begin{array}{l}\text { Mostly } \\
\text { A's }\end{array}$ & $\begin{array}{l}\text { Mostly A's } \\
\& \text { B's }\end{array}$ & $\begin{array}{l}\text { Mostly } \\
\text { B's }\end{array}$ & $\begin{array}{l}\text { Mostly B's } \\
\text { \& C's }\end{array}$ & $\begin{array}{l}\text { Mostly } \\
\text { C's }\end{array}$ & $\begin{array}{l}\text { Mostly C's } \\
\text { \& D's }\end{array}$ & $\begin{array}{l}\text { Mostly D's } \\
\text { and Lower }\end{array}$ \\
\hline Youth Poll 1 (Apr 01) & QNA & QNA & QNA & QNA & QNA & QNA & QNA \\
\hline Youth Poll 2 (Aug 01) & QNA & QNA & QNA & QNA & QNA & QNA & QNA \\
\hline Youth Poll 3 (Nov 01) & 8.2 & 8.6 & 8.7 & 8.7 & $\ddagger$ & $\ddagger$ & $\ddagger$ \\
\hline Youth Poll 4 (Nov 02) & 7.8 & 7.8 & 7.6 & 7.4 & 7.4 & $\ddagger$ & $\ddagger$ \\
\hline Youth Poll 5 (June 03) & 7.9 & 8.0 & 7.9 & 7.8 & 7.3 & 7.7 & $\ddagger$ \\
\hline Youth Poll 6 (Nov 03) & 7.6 & 7.6 & 7.4 & 7.5 & 7.3 & 7.6 & $\ddagger$ \\
\hline Youth Poll 7 (May 04) & 7.8 & 7.6 & 7.5 & 7.5 & 7.1 & 7.4 & $\ddagger$ \\
\hline Youth Poll 8 (Nov 04) & 7.8 & 7.5 & 7.2 & 7.4 & 7.2 & 7.2 & $\ddagger$ \\
\hline \multicolumn{8}{|l|}{ Male (age 16-21) } \\
\hline (mean) & \multicolumn{7}{|c|}{ High School Grades } \\
\hline Year & $\begin{array}{l}\text { Mostly } \\
\text { A's }\end{array}$ & $\begin{array}{l}\text { Mostly A's } \\
\text { \& B's }\end{array}$ & $\begin{array}{l}\text { Mostly } \\
\text { B's }\end{array}$ & $\begin{array}{l}\text { Mostly B's } \\
\text { \& C'S }\end{array}$ & $\begin{array}{l}\text { Mostly } \\
\text { C's }\end{array}$ & $\begin{array}{l}\text { Mostly C's } \\
\text { \& D's }\end{array}$ & $\begin{array}{l}\text { Mostly D's } \\
\text { and Lower }\end{array}$ \\
\hline Youth Poll 1 (Apr 01) & QNA & QNA & QNA & QNA & QNA & QNA & QNA \\
\hline Youth Poll 2 (Aug 01) & QNA & QNA & QNA & QNA & QNA & QNA & QNA \\
\hline Youth Poll 3 (Nov 01) & 8.4 & 8.6 & 8.7 & 8.6 & $\ddagger$ & $\ddagger$ & $\ddagger$ \\
\hline Youth Poll 4 (Nov 02) & $\ddagger$ & 7.6 & 7.5 & 7.3 & $\ddagger$ & $\ddagger$ & $\ddagger$ \\
\hline Youth Poll 5 (June 03) & 8.0 & 7.9 & 7.9 & 7.7 & 7.3 & 7.4 & $\ddagger$ \\
\hline Youth Poll 6 (Nov 03) & 7.3 & 7.7 & 7.7 & 7.5 & 7.1 & 7.5 & $\ddagger$ \\
\hline Youth Poll 7 (May 04) & 7.7 & 7.6 & 7.4 & 7.7 & $\ddagger$ & 7.6 & $\ddagger$ \\
\hline Youth Poll 8 (Nov 04) & 7.7 & 7.5 & 7.4 & 7.4 & 7.3 & $\ddagger$ & $\ddagger$ \\
\hline
\end{tabular}

Females (age 16-21)

(mean)

High School Grades

\begin{tabular}{lccccccc} 
& $\begin{array}{c}\text { Mostly } \\
\text { A's }\end{array}$ & $\begin{array}{c}\text { Mostly A's } \\
\text { \& B's }\end{array}$ & $\begin{array}{c}\text { Mostly } \\
\text { B's }\end{array}$ & $\begin{array}{c}\text { Mostly B's } \\
\text { \& C's }\end{array}$ & $\begin{array}{c}\text { Mostly } \\
\text { C's }\end{array}$ & $\begin{array}{c}\text { Mostly C's } \\
\text { \& D's }\end{array}$ & $\begin{array}{c}\text { Mostly D's } \\
\text { and Lower }\end{array}$ \\
\hline Youth Poll 1 (Apr 01) & QNA & QNA & QNA & QNA & QNA & QNA & QNA \\
Youth Poll 2 (Aug 01) & QNA & QNA & QNA & QNA & QNA & QNA & QNA \\
Youth Poll 3 (Nov 01) & 8.1 & 8.6 & 8.6 & 8.9 & $\ddagger$ & $\ddagger$ & $\ddagger$ \\
Youth Poll 4 (Nov 02) & 7.9 & 7.9 & 7.7 & 7.6 & $\ddagger$ & $\ddagger$ & $\ddagger$ \\
Youth Poll 5 (June 03) & 7.8 & 8.0 & 7.8 & 7.9 & $\ddagger$ & $\ddagger$ & $\ddagger$ \\
Youth Poll 6 (Nov 03) & 7.8 & 7.6 & 7.1 & 7.6 & $\ddagger$ & $\ddagger$ & $\ddagger$ \\
Youth Poll 7 (May 04) & 7.8 & 7.6 & 7.5 & 7.1 & $\ddagger$ & $\ddagger$ & $\ddagger$ \\
Youth Poll 8 (Nov 04) & 7.8 & 7.5 & 7.1 & 7.3 & $\ddagger$ & $\ddagger$ & $\ddagger$
\end{tabular}

80 ‡Reporting standard not met (too few cases); QNA: Question Not Asked

Note: Changes in sample size and stratification were implemented following November 2002 Youth Poll.

Source: Department of Defense Polls, JAMRS, 2001-2004 (Question: FAV2A). 


\section{Coast Guard Favorability}

TABLE 17-1. Youth Coast Guard favorability: $2001-2004^{81}$

\begin{tabular}{lc}
$\begin{array}{l}\text { Male and Female (age 16-21) } \\
\text { (mean) }\end{array}$ & \\
\cline { 2 - 2 } Year & Mean \\
\hline Youth Poll 1 (Apr 01) & QNA \\
Youth Poll 2 (Aug 01) & QNA \\
Youth Poll 3 (Nov 01) & 8.0 \\
Youth Poll 4 (Nov 02) & 6.8 \\
Youth Poll 5 (June 03) & 7.2 \\
Youth Poll 6 (Nov 03) & 6.8 \\
Youth Poll 7 (May 04) & 6.8 \\
Youth Poll 8 (Nov 04) & 6.7 \\
\hline
\end{tabular}

\begin{tabular}{lc}
$\begin{array}{l}\text { Male (age 16-21) } \\
\text { (mean) }\end{array}$ \\
\cline { 2 - 2 } Year & Mean \\
\hline Youth Poll 1 (Apr 01) & QNA \\
Youth Poll 2 (Aug 01) & QNA \\
Youth Poll 3 (Nov 01) & 7.9 \\
Youth Poll 4 (Nov 02) & 6.5 \\
Youth Poll 5 (June 03) & 6.9 \\
Youth Poll 6 (Nov 03) & 6.6 \\
Youth Poll 7 (May 04) & 6.8 \\
Youth Poll 8 (Nov 04) & 6.7 \\
\hline
\end{tabular}

\begin{tabular}{lc}
$\begin{array}{l}\text { Female (age 16-21) } \\
\text { (mean) }\end{array}$ & \\
\cline { 2 - 2 } Year & Mean \\
\hline Youth Poll 1 (Apr 01) & QNA \\
Youth Poll 2 (Aug 01) & QNA \\
Youth Poll 3 (Nov 01) & 8.2 \\
Youth Poll 4 (Nov 02) & 7.1 \\
Youth Poll 5 (June 03) & 7.4 \\
Youth Poll 6 (Nov 03) & 7.0 \\
Youth Poll 7 (May 04) & 6.8 \\
Youth Poll 8 (Nov 04) & 6.8 \\
\hline
\end{tabular}

${ }^{81}$ ‡Reporting standard not met (too few cases); QNA: Question Not Asked Note: Changes in sample size and stratification were implemented following November 2002 Youth Poll. Source: Department of Defense Polls, JAMRS, 2001-2004 (Question: FAV2C). 


\section{Coast Guard Favorability}

TABLE 17-2. Youth Coast Guard favorability, by race/ethnicity: $2001-2004^{82}$

\begin{tabular}{lccc} 
Male and Female (age 16-21) & \multicolumn{3}{c}{ Racelethnicity } \\
\cline { 2 - 4 } (mean) & White & Black & Hispanic \\
\cline { 2 - 4 } Year & QNA & QNA & QNA \\
\hline Youth Poll 1 (Apr 01) & QNA & QNA & QNA \\
Youth Poll 2 (Aug 01) & 8.1 & 7.8 & 7.9 \\
Youth Poll 3 (Nov 01) & 7.0 & 6.5 & 6.6 \\
Youth Poll 4 (Nov 02) & 7.4 & 6.3 & 7.1 \\
Youth Poll 5 (June 03) & 7.0 & 6.1 & 6.7 \\
Youth Poll 6 (Nov 03) & 7.0 & 6.0 & 6.6 \\
Youth Poll 7 (May 04) & 7.0 & 5.6 & 6.7 \\
Youth Poll 8 (Nov 04) & & & \\
\hline
\end{tabular}

\begin{tabular}{lccc} 
Male (age 16-21) & \multicolumn{3}{c}{ Racelethnicity } \\
\cline { 2 - 4 } (mean) & White & Black & Hispanic \\
\cline { 2 - 4 } Year & QNA & QNA & QNA \\
\hline Youth Poll 1 (Apr 01) & QNA & QNA & QNA \\
Youth Poll 2 (Aug 01) & 7.9 & 8.0 & 7.8 \\
Youth Poll 3 (Nov 01) & 6.6 & 6.4 & 6.4 \\
Youth Poll 4 (Nov 02) & 7.1 & 6.3 & 6.8 \\
Youth Poll 5 (June 03) & 6.8 & 6.1 & 6.6 \\
Youth Poll 6 (Nov 03) & 7.0 & 6.0 & 6.4 \\
Youth Poll 7 (May 04) & 7.0 & 5.7 & 6.5 \\
Youth Poll 8 (Nov 04) & & &
\end{tabular}

\begin{tabular}{|c|c|c|c|}
\hline \multicolumn{4}{|l|}{ Female (age 16-21) } \\
\hline \multirow{2}{*}{$\begin{array}{l}\text { (mean) } \\
\text { Year }\end{array}$} & \multicolumn{3}{|c|}{ Race/ethnicity } \\
\hline & White & Black & Hispanic \\
\hline Youth Poll 1 (Apr 01) & QNA & QNA & QNA \\
\hline Youth Poll 2 (Aug 01) & QNA & QNA & QNA \\
\hline Youth Poll 3 (Nov 01) & 8.3 & 7.7 & 8.0 \\
\hline Youth Poll 4 (Nov 02) & 7.3 & 6.6 & 6.8 \\
\hline Youth Poll 5 (June 03) & 7.7 & 6.4 & 7.4 \\
\hline Youth Poll 6 (Nov 03) & 7.3 & 6.1 & 6.9 \\
\hline Youth Poll 7 (May 04) & 7.0 & 5.9 & 6.8 \\
\hline Youth Poll 8 (Nov 04) & 7.1 & 5.6 & 6.9 \\
\hline
\end{tabular}

${ }^{82}$ Due to relatively small sample sizes, American Indian, Alaska Native, Asian, and Pacific Islander are included in the total but are not shown separately. Black includes African American, and Hispanic includes Latino. Race categories exclude Hispanic origin unless specified otherwise.

†Reporting standard not met (too few cases); QNA: Question Not Asked

Note: Changes in sample size and stratification were implemented following November 2002 Youth Poll.

Source: Department of Defense Polls, JAMRS, 2001-2004 (Question: FAV2C). 


\section{Coast Guard Favorability}

JAMRS

TABLE 17-3. Youth Coast Guard favorability, by age: $2001-2004^{83}$

\begin{tabular}{|c|c|c|c|c|c|c|}
\hline \multirow{3}{*}{$\begin{array}{l}\text { Male and Female (age 16-21) } \\
\text { (mean) } \\
\text { Year }\end{array}$} & & & & & & \\
\hline & \multicolumn{6}{|c|}{ Age } \\
\hline & 16 & 17 & 18 & 19 & 20 & 21 \\
\hline Youth Poll 1 (Apr 01) & QNA & QNA & QNA & QNA & QNA & QNA \\
\hline Youth Poll 2 (Aug 01) & QNA & QNA & QNA & QNA & QNA & QNA \\
\hline Youth Poll 3 (Nov 01) & 7.9 & 7.8 & 8.1 & 8.2 & 8.0 & 8.1 \\
\hline Youth Poll 4 (Nov 02) & 6.7 & 6.6 & 6.7 & 6.8 & 6.7 & 7.4 \\
\hline Youth Poll 5 (June 03) & 7.3 & 7.1 & 6.9 & 7.2 & 7.2 & 7.3 \\
\hline Youth Poll 6 (Nov 03) & 6.7 & 6.6 & 6.8 & 6.8 & 7.0 & 7.1 \\
\hline Youth Poll 7 (May 04) & 6.9 & 6.6 & 6.6 & 7.0 & 6.6 & 6.9 \\
\hline Youth Poll 8 (Nov 04) & 6.7 & 6.6 & 6.8 & 6.6 & 6.8 & 7.0 \\
\hline
\end{tabular}

\begin{tabular}{lcccccc} 
Male (age 16-21) & \multicolumn{7}{c}{ Age } \\
\cline { 2 - 7 } (mean) & $\mathbf{1 6}$ & $\mathbf{1 7}$ & $\mathbf{1 8}$ & $\mathbf{1 9}$ & $\mathbf{2 0}$ & $\mathbf{2 1}$ \\
\cline { 2 - 7 } Year & QNA & QNA & QNA & QNA & QNA & QNA \\
\hline Youth Poll 1 (Apr 01) & QNA & QNA & QNA & QNA & QNA & QNA \\
Youth Poll 2 (Aug 01) & 7.8 & 7.9 & 7.9 & 7.8 & 7.8 & 7.9 \\
Youth Poll 3 (Nov 01) & 6.7 & 6.2 & 6.7 & 6.2 & 6.4 & 7.2 \\
Youth Poll 4 (Nov 02) & 7.0 & 6.9 & 6.6 & 7.0 & 6.9 & 7.2 \\
Youth Poll 5 (June 03) & 6.5 & 6.4 & 6.6 & 6.7 & 6.7 & 6.9 \\
Youth Poll 6 (Nov 03) & 6.8 & 6.8 & 6.5 & 7.0 & 6.4 & 7.1 \\
Youth Poll 7 (May 04) & 6.7 & 6.5 & 6.9 & 6.6 & 6.9 & 6.8 \\
Youth Poll 8 (Nov 04) & & & & & 6.9 & \\
\hline
\end{tabular}

\section{Female (age 16-21)}

(mean)

Year

Youth Poll 1 (Apr 01)

Youth Poll 2 (Aug 01)

Youth Poll 3 (Nov 01)

Youth Poll 4 (Nov 02)

Youth Poll 5 (June 03)

Youth Poll 6 (Nov 03)

Youth Poll 7 (May 04)

\begin{tabular}{cccccc}
\hline \multicolumn{7}{c}{ Age } \\
\hline $\mathbf{1 6}$ & $\mathbf{1 7}$ & $\mathbf{1 8}$ & $\mathbf{1 9}$ & $\mathbf{2 0}$ & $\mathbf{2 1}$ \\
QNA & QNA & QNA & QNA & QNA & QNA \\
QNA & QNA & QNA & QNA & QNA & QNA \\
8.0 & 7.7 & 8.3 & 8.5 & 8.2 & 8.2 \\
6.8 & 6.9 & 6.6 & 7.4 & 7.1 & 7.6 \\
7.5 & 7.5 & 7.1 & 7.4 & 7.5 & 7.5 \\
6.9 & 6.8 & 7.0 & 7.0 & 7.3 & 7.3 \\
7.0 & 6.5 & 6.8 & 7.0 & 6.9 & 6.7 \\
6.8 & 6.7 & 6.7 & 6.6 & 6.6 & 7.1 \\
\hline
\end{tabular}

Youth Poll 8 (Nov 04)

${ }^{83}$ ‡Reporting standard not met (too few cases); QNA: Question Not Asked

Note: Changes in sample size and stratification were implemented following November 2002 Youth Poll.

Source: Department of Defense Polls, JAMRS, 2001-2004 (Question: FAV2C).

Page A-83 DoD May 2004 Youth Poll 


\section{Coast Guard Favorability}

JAMRS

TABLE 17-4. Youth Coast Guard favorability, by geographic region: $2001-2004^{84}$

\section{Male and Female (age 16-21)}

(mean)

Year

Youth Poll 1 (Apr 01)

Youth Poll 2 (Aug 01)

Youth Poll 3 (Nov 01)

Youth Poll 4 (Nov 02)

Youth Poll 5 (June 03)

\begin{tabular}{ccccccccc}
\hline \multicolumn{10}{c}{ Geographic Region } \\
$\begin{array}{c}\text { New } \\
\text { England }\end{array}$ & $\begin{array}{c}\text { Mid } \\
\text { Atlantic }\end{array}$ & $\begin{array}{c}\text { East } \\
\text { North } \\
\text { Central }\end{array}$ & $\begin{array}{c}\text { West } \\
\text { North } \\
\text { Central }\end{array}$ & $\begin{array}{c}\text { South } \\
\text { Atlantic }\end{array}$ & $\begin{array}{c}\text { East } \\
\text { South } \\
\text { Central }\end{array}$ & $\begin{array}{c}\text { West } \\
\text { South } \\
\text { Central }\end{array}$ & Mountain & Pacific \\
QNA & QNA & QNA & QNA & QNA & QNA & QNA & QNA & QNA \\
QNA & QNA & QNA & QNA & QNA & QNA & QNA & QNA & QNA \\
$\ddagger$ & $\ddagger$ & $\ddagger$ & $\ddagger$ & $\ddagger$ & $\ddagger$ & $\ddagger$ & $\ddagger$ & $\ddagger$ \\
$\ddagger$ & $\ddagger$ & $\ddagger$ & $\ddagger$ & $\ddagger$ & $\ddagger$ & $\ddagger$ & $\ddagger$ & $\ddagger$ \\
7.0 & 7.4 & 7.3 & 7.4 & 7.3 & 6.9 & 7.0 & 7.0 & 7.0 \\
7.2 & 6.7 & 6.7 & 6.9 & 6.8 & 7.0 & 6.6 & 7.1 & 6.9 \\
7.4 & 6.6 & 6.7 & 6.8 & 7.1 & 7.0 & 6.6 & 6.8 & 6.6 \\
6.4 & 6.5 & 6.6 & 6.9 & 6.9 & 6.9 & 6.8 & 7.1 & 6.7 \\
\hline
\end{tabular}

Youth Poll 6 (Nov 03)

Youth Poll 7 (May 04)

6.5

6.6

\section{Geographic Region}

(mean)

Year

New
England

Mid

East West

Atlantic

$\begin{array}{cc}\text { East } & \text { West } \\ \text { North } & \text { North }\end{array}$

Central Central

South

East

Atlantic

South South

Central Central

QNA Pacific

Youth Poll 1 (Apr 01)

Youth Poll 2 (Aug 01)

Youth Poll 3 (Nov 01)

Youth Poll 4 (Nov 02)

Youth Poll 5 (June 03)

Youth Poll 6 (Nov 03)

Youth Poll 7 (May 04)

QNA

Youth Poll 8 (Nov 04)

QNA

QNA QNA QNA

QNA

QNA

QNA

F

$\ddagger$

‡

QNA

QNA

QNA

QNA

QNA

$\ddagger$

$\ddagger$

$\ddagger$

‡

$\ddagger$

7.2

7.1

$\ddagger$

$\ddagger$

‡

$\ddagger$

6.7

6.4

6.9

7.0

$\ddagger$

$\ddagger$

$\ddagger \quad 6.6$

6.8

6.4

6.6

6.6

6.7

$\ddagger$

6.9

7.0

6.8

\section{$\ddagger$}

$\ddagger$

6.9

6.3

QNA

QNA

†

$\ddagger$

6.6

$\neq$

6.5

6.5

$\ddagger$

6.9

\section{Geographic Region}

(mean)

\section{Year}

Youth Poll 1 (Apr 01)

Youth Poll 2 (Aug 01)

Youth Poll 3 (Nov 01)

Youth Poll 4 (Nov 02)

Youth Poll 5 (June 03)

Youth Poll 6 (Nov 03)

Youth Poll 7 (May 04)

Youth Poll 8 (Nov 04)

\begin{tabular}{ccccccccc}
\hline \multicolumn{10}{c}{ Geographic Region } \\
$\begin{array}{c}\text { New } \\
\text { England }\end{array}$ & $\begin{array}{c}\text { Mid } \\
\text { Atlantic }\end{array}$ & $\begin{array}{c}\text { East } \\
\text { North } \\
\text { Central }\end{array}$ & $\begin{array}{c}\text { West } \\
\text { North } \\
\text { Central }\end{array}$ & $\begin{array}{c}\text { South } \\
\text { Atlantic }\end{array}$ & $\begin{array}{c}\text { East } \\
\text { South } \\
\text { Central }\end{array}$ & $\begin{array}{c}\text { West } \\
\text { South } \\
\text { Central }\end{array}$ & Mountain & Pacific \\
QNA & QNA & QNA & QNA & QNA & QNA & QNA & QNA & QNA \\
QNA & QNA & QNA & QNA & QNA & QNA & QNA & QNA & QNA \\
$\ddagger$ & $\ddagger$ & $\ddagger$ & $\ddagger$ & $\ddagger$ & $\ddagger$ & $\ddagger$ & $\ddagger$ & $\ddagger$ \\
$\ddagger$ & $\ddagger$ & $\ddagger$ & $\ddagger$ & $\ddagger$ & $\ddagger$ & $\ddagger$ & $\ddagger$ & $\ddagger$ \\
$\ddagger$ & 7.5 & 7.5 & 7.8 & 7.5 & $\ddagger$ & 7.1 & 7.5 & 7.3 \\
$\ddagger$ & 6.7 & 6.9 & 7.5 & 7.1 & 7.0 & 6.8 & 7.1 & 7.1 \\
$\ddagger$ & 6.6 & 6.6 & $\ddagger$ & 7.2 & $\ddagger$ & 6.7 & $\ddagger$ & 6.7 \\
$\ddagger$ & 6.3 & 6.5 & 6.8 & 6.9 & $\ddagger$ & 7.0 & 7.4 & 6.8
\end{tabular}

\footnotetext{
${ }^{84} \ddagger$ Reporting standard not met (too few cases); QNA: Question Not Asked

Note: Changes in sample size and stratification were implemented following November 2002 Youth Poll.

Source: Department of Defense Polls, JAMRS, 2001-2004 (Question: FAV2C).
} 


\section{Coast Guard Favorability}

JAMRS

TABLE 17-5. Youth Coast Guard favorability, by high school grades: $2001-2004^{85}$

\begin{tabular}{lccccccc} 
Male and Female (age 16-21) \\
\cline { 2 - 7 } (mean) & $\begin{array}{c}\text { Mostly } \\
\text { A's }\end{array}$ & $\begin{array}{c}\text { Mostly A's } \\
\text { \& B's }\end{array}$ & $\begin{array}{c}\text { Mostly } \\
\text { B's }\end{array}$ & $\begin{array}{c}\text { Mostly B's } \\
\text { \& C's }\end{array}$ & $\begin{array}{c}\text { Mostly } \\
\text { C's }\end{array}$ & $\begin{array}{c}\text { Mostly C's } \\
\text { \& D's }\end{array}$ & $\begin{array}{c}\text { Mostly D's } \\
\text { and Lower }\end{array}$ \\
\cline { 2 - 8 } Year & QNA & QNA & QNA & QNA & QNA & QNA & QNA \\
\hline Youth Poll 1 (Apr 01) & QNA & QNA & QNA & QNA & QNA & QNA & QNA \\
Youth Poll 2 (Aug 01) & 7.9 & 8.1 & 8.0 & 8.1 & $\ddagger$ & $\ddagger$ & $\ddagger$ \\
Youth Poll 3 (Nov 01) & 7.0 & 7.0 & 7.0 & 6.6 & 6.4 & $\ddagger$ & $\ddagger$ \\
Youth Poll 4 (Nov 02) & 7.4 & 7.4 & 7.3 & 6.9 & 6.5 & 6.9 & $\ddagger$ \\
Youth Poll 5 (June 03) & 7.1 & 7.0 & 6.8 & 6.7 & 6.1 & 6.4 & $\ddagger$ \\
Youth Poll 6 (Nov 03) & 7.1 & 6.9 & 6.5 & 6.6 & 6.3 & 6.6 & $\ddagger$ \\
Youth Poll 7 (May 04) & 7.2 & 6.8 & 6.5 & 6.6 & 6.4 & 6.2 & $\ddagger$ \\
Youth Poll 8 (Nov 04) & 7.2 &
\end{tabular}

\begin{tabular}{lccccccc} 
Male (age 16-21) & \multicolumn{7}{c}{ High School Grades } \\
\cline { 2 - 7 } (mean) & $\begin{array}{c}\text { Mostly } \\
\text { A's }\end{array}$ & $\begin{array}{c}\text { Mostly A's } \\
\text { \& B's }\end{array}$ & $\begin{array}{c}\text { Mostly } \\
\text { B's }\end{array}$ & $\begin{array}{c}\text { Mostly B's } \\
\text { \& C's }\end{array}$ & $\begin{array}{c}\text { Mostly } \\
\text { C's }\end{array}$ & $\begin{array}{c}\text { Mostly C's } \\
\text { \& D's }\end{array}$ & $\begin{array}{c}\text { Mostly D's } \\
\text { and Lower }\end{array}$ \\
\cline { 2 - 8 } Year & QNA & QNA & QNA & QNA & QNA & QNA & QNA \\
\hline Youth Poll 1 (Apr 01) & QNA & QNA & QNA & QNA & QNA & QNA & QNA \\
Youth Poll 2 (Aug 01) & 8.1 & 8.0 & 7.8 & 7.8 & $\ddagger$ & $\ddagger$ & $\ddagger$ \\
Youth Poll 3 (Nov 01) & $\ddagger$ & 6.8 & 6.6 & 6.6 & $\ddagger$ & $\ddagger$ & $\ddagger$ \\
Youth Poll 4 (Nov 02) & 7.2 & 7.2 & 7.1 & 6.8 & 6.3 & 6.5 & $\ddagger$ \\
Youth Poll 5 (June 03) & 6.7 & 6.9 & 6.8 & 6.6 & 6.0 & 6.3 & $\ddagger$ \\
Youth Poll 6 (Nov 03) & 6.8 & 7.0 & 6.3 & 6.8 & $\ddagger$ & 6.8 & $\ddagger$ \\
Youth Poll 7 (May 04) & 7.2 & 6.9 & 6.5 & 6.6 & 6.5 & $\ddagger$ & $\ddagger$ \\
Youth Poll 8 (Nov 04) & & & & & &
\end{tabular}

\section{Females (age 16-21)}

(mean)

High School Grades

\begin{tabular}{lccccccc}
\cline { 2 - 7 } Year & $\begin{array}{c}\text { Mostly } \\
\text { A's }\end{array}$ & $\begin{array}{c}\text { Mostly A's } \\
\text { \& B's }\end{array}$ & $\begin{array}{c}\text { Mostly } \\
\text { B's }\end{array}$ & $\begin{array}{c}\text { Mostly B's } \\
\text { \& C's }\end{array}$ & $\begin{array}{c}\text { Mostly } \\
\text { C's }\end{array}$ & $\begin{array}{c}\text { Mostly C's } \\
\text { \& D's }\end{array}$ & $\begin{array}{c}\text { Mostly D's } \\
\text { and Lower }\end{array}$ \\
\hline Youth Poll 1 (Apr 01) & QNA & QNA & QNA & QNA & QNA & QNA & QNA \\
Youth Poll 2 (Aug 01) & QNA & QNA & QNA & QNA & QNA & QNA & QNA \\
Youth Poll 3 (Nov 01) & 7.8 & 8.1 & 8.2 & 8.4 & $\ddagger$ & $\ddagger$ & $\ddagger$ \\
Youth Poll 4 (Nov 02) & 7.3 & 7.1 & 7.3 & 6.8 & $\ddagger$ & $\ddagger$ & $\ddagger$ \\
Youth Poll 5 (June 03) & 7.5 & 7.6 & 7.5 & 7.1 & $\ddagger$ & $\ddagger$ & $\ddagger$ \\
Youth Poll 6 (Nov 03) & 7.3 & 7.1 & 6.8 & 6.9 & $\ddagger$ & $\ddagger$ & $\ddagger$ \\
Youth Poll 7 (May 04) & 7.3 & 6.9 & 6.8 & 6.5 & $\ddagger$ & $\ddagger$ & $\ddagger$ \\
Youth Poll 8 (Nov 04) & 7.3 & 6.8 & 6.5 & 6.7 & $\ddagger$ & $\ddagger$ & $\ddagger$ \\
\hline
\end{tabular}

${ }^{85} \ddagger$ Reporting standard not met (too few cases); QNA: Question Not Asked

Note: Changes in sample size and stratification were implemented following November 2002 Youth Poll.

Source: Department of Defense Polls, JAMRS, 2001-2004 (Question: FAV2C). 


\section{Reserve Favorability}

TABLE 18-1. Youth Reserve favorability: $2001-2004^{86}$

\begin{tabular}{lc}
$\begin{array}{l}\text { Male and Female (age 16-21) } \\
\text { (mean) }\end{array}$ & \\
\cline { 2 - 2 } Year & Mean \\
\hline Youth Poll 1 (Apr 01) & QNA \\
Youth Poll 2 (Aug 01) & QNA \\
Youth Poll 3 (Nov 01) & 8.2 \\
Youth Poll 4 (Nov 02) & 7.0 \\
Youth Poll 5 (June 03) & 7.3 \\
Youth Poll 6 (Nov 03) & 7.2 \\
Youth Poll 7 (May 04) & 7.0 \\
Youth Poll 8 (Nov 04) & 6.9 \\
\hline
\end{tabular}

\begin{tabular}{lc}
$\begin{array}{l}\text { Male (age 16-21) } \\
\text { (mean) }\end{array}$ \\
\cline { 2 - 2 } Year & Mean \\
\hline Youth Poll 1 (Apr 01) & QNA \\
Youth Poll 2 (Aug 01) & QNA \\
Youth Poll 3 (Nov 01) & 8.0 \\
Youth Poll 4 (Nov 02) & 6.7 \\
Youth Poll 5 (June 03) & 7.1 \\
Youth Poll 6 (Nov 03) & 7.0 \\
Youth Poll 7 (May 04) & 6.9 \\
Youth Poll 8 (Nov 04) & 6.8 \\
\hline
\end{tabular}

\begin{tabular}{lc}
$\begin{array}{l}\text { Female (age 16-21) } \\
\text { (mean) }\end{array}$ & \\
\cline { 2 - 2 } Year & Mean \\
\hline Youth Poll 1 (Apr 01) & QNA \\
Youth Poll 2 (Aug 01) & QNA \\
Youth Poll 3 (Nov 01) & 8.3 \\
Youth Poll 4 (Nov 02) & 7.3 \\
Youth Poll 5 (June 03) & 7.6 \\
Youth Poll 6 (Nov 03) & 7.4 \\
Youth Poll 7 (May 04) & 7.1 \\
Youth Poll 8 (Nov 04) & 7.1 \\
\hline
\end{tabular}

${ }^{86}$ ‡Reporting standard not met (too few cases); QNA: Question Not Asked Note: Changes in sample size and stratification were implemented following November 2002 Youth Poll. Source: Department of Defense Polls, JAMRS, 2001-2004 (Question: FAV3A). 


\section{Reserve Favorability}

TABLE 18-2. Youth Reserve favorability, by race/ethnicity: $2001-2004^{87}$

\begin{tabular}{lccc} 
Male and Female (age 16-21) & \multicolumn{3}{c}{ Racelethnicity } \\
\cline { 2 - 4 } (mean) & White & Black & Hispanic \\
\cline { 2 - 4 } Year & QNA & QNA & QNA \\
\hline Youth Poll 1 (Apr 01) & QNA & QNA & QNA \\
Youth Poll 2 (Aug 01) & 8.1 & 8.4 & 8.1 \\
Youth Poll 3 (Nov 01) & 7.1 & 7.0 & 6.9 \\
Youth Poll 4 (Nov 02) & 7.5 & 6.6 & 7.3 \\
Youth Poll 5 (June 03) & 7.3 & 6.8 & 7.2 \\
Youth Poll 6 (Nov 03) & 7.1 & 6.3 & 6.9 \\
Youth Poll 7 (May 04) & 7.2 & 6.0 & 6.9 \\
Youth Poll 8 (Nov 04) & & & \\
\hline
\end{tabular}

\begin{tabular}{lccc} 
Male (age 16-21) & \multicolumn{3}{c}{ Racelethnicity } \\
\cline { 2 - 4 } (mean) & White & Black & Hispanic \\
\cline { 2 - 4 } Year & QNA & QNA & QNA \\
\hline Youth Poll 1 (Apr 01) & QNA & QNA & QNA \\
Youth Poll 2 (Aug 01) & 7.9 & 8.3 & 8.0 \\
Youth Poll 3 (Nov 01) & 6.7 & 7.0 & 6.5 \\
Youth Poll 4 (Nov 02) & 7.2 & 6.5 & 6.9 \\
Youth Poll 5 (June 03) & 7.1 & 6.8 & 7.0 \\
Youth Poll 6 (Nov 03) & 7.0 & 6.5 & 6.7 \\
Youth Poll 7 (May 04) & 7.0 & 5.9 & 6.6 \\
Youth Poll 8 (Nov 04) & & & \\
\hline
\end{tabular}

\begin{tabular}{lccc} 
Female (age 16-21) & \multicolumn{3}{c}{ Racelethnicity } \\
\cline { 2 - 4 } (mean) & White & Black & Hispanic \\
\cline { 2 - 4 } Year & QNA & QNA & QNA \\
\hline Youth Poll 1 (Apr 01) & QNA & QNA & QNA \\
Youth Poll 2 (Aug 01) & 8.4 & 8.5 & 8.1 \\
Youth Poll 3 (Nov 01) & 7.4 & 7.0 & 7.2 \\
Youth Poll 4 (Nov 02) & 7.8 & 6.8 & 7.7 \\
Youth Poll 5 (June 03) & 7.5 & 6.8 & 7.5 \\
Youth Poll 6 (Nov 03) & 7.2 & 6.2 & 7.1 \\
Youth Poll 7 (May 04) & 7.4 & 6.0 & 7.2 \\
Youth Poll 8 (Nov 04) & & & \\
\hline
\end{tabular}

${ }^{87}$ Due to relatively small sample sizes, American Indian, Alaska Native, Asian, and Pacific Islander are included in the total but are not shown separately. Black includes African American, and Hispanic includes Latino. Race categories exclude Hispanic origin unless specified otherwise.

†Reporting standard not met (too few cases); QNA: Question Not Asked

Note: Changes in sample size and stratification were implemented following November 2002 Youth Poll.

Source: Department of Defense Polls, JAMRS, 2001-2004 (Question: FAV3A). 


\section{Reserve Favorability}

JAMRS

TABLE 18-3. Youth Reserve favorability, by age: $2001-2004^{88}$

\begin{tabular}{lcccccc} 
Male and Female (age 16-21) & \multicolumn{7}{c}{ Age } \\
\cline { 2 - 7 } (mean) & 16 & $\mathbf{1 7}$ & $\mathbf{1 8}$ & $\mathbf{1 9}$ & $\mathbf{2 0}$ & $\mathbf{2 1}$ \\
\cline { 2 - 7 } Year & QNA & QNA & QNA & QNA & QNA & QNA \\
\cline { 2 - 7 } Youth Poll 1 (Apr 01) & QNA & QNA & QNA & QNA & QNA & QNA \\
Youth Poll 2 (Aug 01) & 8.1 & 8.1 & 8.2 & 8.4 & 8.1 & 8.1 \\
Youth Poll 3 (Nov 01) & 7.1 & 7.0 & 6.7 & 7.0 & 6.9 & 7.5 \\
Youth Poll 4 (Nov 02) & 7.4 & 7.3 & 7.3 & 7.3 & 7.3 & 7.4 \\
Youth Poll 5 (June 03) & 7.1 & 7.1 & 7.2 & 7.2 & 7.5 & 7.0 \\
Youth Poll 6 (Nov 03) & 7.1 & 7.0 & 6.9 & 7.0 & 6.8 & 6.9 \\
Youth Poll 7 (May 04) & 7.1 & 7.0 & 7.1 & 6.6 & 7.0 & 6.9 \\
Youth Poll 8 (Nov 04) & & & & & & \\
\hline
\end{tabular}

\begin{tabular}{lcccccc} 
Male (age 16-21) & \multicolumn{7}{c}{ Age } \\
\cline { 2 - 7 } (mean) & $\mathbf{1 6}$ & $\mathbf{1 7}$ & $\mathbf{1 8}$ & $\mathbf{1 9}$ & $\mathbf{2 0}$ & $\mathbf{2 1}$ \\
\cline { 2 - 7 } Year & QNA & QNA & QNA & QNA & QNA & QNA \\
\hline Youth Poll 1 (Apr 01) & QNA & QNA & QNA & QNA & QNA & QNA \\
Youth Poll 2 (Aug 01) & 8.0 & 8.1 & 7.9 & 8.2 & 7.8 & 7.8 \\
Youth Poll 3 (Nov 01) & 7.0 & 6.7 & 6.6 & 6.2 & 6.6 & 7.4 \\
Youth Poll 4 (Nov 02) & 7.3 & 6.9 & 6.9 & 7.2 & 6.8 & 7.4 \\
Youth Poll 5 (June 03) & 6.9 & 6.8 & 7.0 & 7.0 & 7.3 & 7.0 \\
Youth Poll 6 (Nov 03) & 6.9 & 7.1 & 6.7 & 6.9 & 6.6 & 6.9 \\
Youth Poll 7 (May 04) & 7.1 & 6.8 & 7.0 & 6.4 & 6.9 & 6.5 \\
Youth Poll 8 (Nov 04) & & & & & 6.9 & \\
\hline
\end{tabular}

\section{Female (age 16-21)}

(mean)

Year

Youth Poll 1 (Apr 01)

Youth Poll 2 (Aug 01)

Youth Poll 3 (Nov 01)

Youth Poll 4 (Nov 02)

Youth Poll 5 (June 03)

Youth Poll 6 (Nov 03)

Youth Poll 7 (May 04)

\begin{tabular}{cccccc}
\hline \multicolumn{7}{c}{ Age } \\
\hline $\mathbf{1 6}$ & $\mathbf{1 7}$ & $\mathbf{1 8}$ & $\mathbf{1 9}$ & $\mathbf{2 0}$ & $\mathbf{2 1}$ \\
QNA & QNA & QNA & QNA & QNA & QNA \\
QNA & QNA & QNA & QNA & QNA & QNA \\
8.2 & 8.1 & 8.5 & 8.6 & 8.4 & 8.3 \\
7.2 & 7.3 & 6.8 & 7.7 & 7.3 & 7.6 \\
7.5 & 7.7 & 7.7 & 7.5 & 7.7 & 7.5 \\
7.2 & 7.4 & 7.5 & 7.4 & 7.6 & 7.1 \\
7.3 & 6.9 & 7.3 & 7.2 & 7.0 & 6.8 \\
7.1 & 7.2 & 7.1 & 6.8 & 7.0 & 7.3 \\
\hline
\end{tabular}

Youth Poll 8 (Nov 04)

${ }^{88}$ ‡Reporting standard not met (too few cases); QNA: Question Not Asked

Note: Changes in sample size and stratification were implemented following November 2002 Youth Poll.

Source: Department of Defense Polls, JAMRS, 2001-2004 (Question: FAV3A).

Page A-88 DoD May 2004 Youth Poll 


\section{Reserve Favorability}

JAMRS

TABLE 18-4. Youth Reserve favorability, by geographic region: $2001-2004^{89}$

\section{Male and Female (age 16-21)}

(mean)

Year

Youth Poll 1 (Apr 01)

Youth Poll 2 (Aug 01)

Youth Poll 3 (Nov 01)

Youth Poll 4 (Nov 02)

\begin{tabular}{ccccccccc}
\hline \multicolumn{10}{c}{ Geographic Region } \\
\hline $\begin{array}{c}\text { New } \\
\text { England }\end{array}$ & $\begin{array}{c}\text { Mid } \\
\text { Atlantic }\end{array}$ & $\begin{array}{c}\text { East } \\
\text { North } \\
\text { Central }\end{array}$ & $\begin{array}{c}\text { West } \\
\text { North } \\
\text { Central }\end{array}$ & $\begin{array}{c}\text { South } \\
\text { Atlantic }\end{array}$ & $\begin{array}{c}\text { East } \\
\text { South } \\
\text { Central }\end{array}$ & $\begin{array}{c}\text { West } \\
\text { South } \\
\text { Central }\end{array}$ & Mountain & Pacific \\
QNA & QNA & QNA & QNA & QNA & QNA & QNA & QNA & QNA \\
QNA & QNA & QNA & QNA & QNA & QNA & QNA & QNA & QNA \\
$\ddagger$ & $\ddagger$ & $\ddagger$ & $\ddagger$ & $\ddagger$ & $\ddagger$ & $\ddagger$ & $\ddagger$ & $\ddagger$ \\
$\ddagger$ & $\ddagger$ & $\ddagger$ & $\ddagger$ & $\ddagger$ & $\ddagger$ & $\ddagger$ & $\ddagger$ & $\ddagger$ \\
7.0 & 7.3 & 7.5 & 7.6 & 7.4 & 7.3 & 7.4 & 7.3 & 7.2 \\
6.9 & 7.2 & 7.2 & 7.4 & 7.2 & 7.4 & 7.1 & 7.2 & 7.1 \\
6.9 & 7.0 & 6.9 & 6.9 & 7.2 & 7.3 & 6.9 & 6.8 & 6.8 \\
6.4 & 6.6 & 6.9 & 7.2 & 7.1 & 7.1 & 7.0 & 7.3 & 6.8 \\
\hline
\end{tabular}

Youth Poll 5 (June 03)

Youth Poll 6 (Nov 03)

Youth Poll 7 (May 04)

6.4

\begin{tabular}{cccccc}
\hline & & \multicolumn{4}{c}{ Geographic Region } \\
\hline $\begin{array}{c}\text { New } \\
\text { England }\end{array}$ & $\begin{array}{c}\text { Mid } \\
\text { Atlantic }\end{array}$ & $\begin{array}{c}\text { East } \\
\text { North } \\
\text { Central }\end{array}$ & $\begin{array}{c}\text { West } \\
\text { North } \\
\text { Central }\end{array}$ & $\begin{array}{c}\text { South } \\
\text { Atlantic }\end{array}$ & $\begin{array}{r}\text { Ea } \\
\text { So } \\
\text { Centh }\end{array}$ \\
QNA & QNA & QNA & QNA & QNA & QNA \\
QNA & QNA & QNA & QNA & QNA & QNA \\
$\ddagger$ & $\ddagger$ & $\ddagger$ & $\ddagger$ & $\ddagger$ & \\
$\ddagger$ & $\ddagger$ & $\ddagger$ & $\ddagger$ & $\ddagger$ & \\
$\ddagger$ & 7.1 & 7.2 & 7.3 & 7.2 & $\ddagger$ \\
$\ddagger$ & 6.9 & 7.0 & 7.2 & 7.0 & \\
$\ddagger$ & 6.9 & 7.1 & $\ddagger$ & 7.0 & \\
$\ddagger$ & 6.5 & 7.1 & 7.1 & 7.0 &
\end{tabular}

East West

South South Mountain Pacific

Year

Youth Poll 1 (Apr 01)

Youth Poll 2 (Aug 01)

Youth Poll 3 (Nov 01)

Youth Poll 4 (Nov 02)

Youth Poll 5 (June 03)

Youth Poll 6 (Nov 03)

Youth Poll 7 (May 04)

6.5

$7.1 \quad 7.1$

QNA QNA

QNA QNA QNA

QNA QNA

$\ddagger \quad \ddagger$

$\ddagger$

QNA

QNA

Youth Poll 8 (Nov 04)

Female (age 16-21)

(mean)

Year

Youth Poll 1 (Apr 01)

Youth Poll 2 (Aug 01)

Youth Poll 3 (Nov 01)

Youth Poll 4 (Nov 02)

Youth Poll 5 (June 03)

Youth Poll 6 (Nov 03)

Youth Poll 7 (May 04)

Youth Poll 8 (Nov 04)

\begin{tabular}{ccccccccc}
\hline \multicolumn{10}{c}{ Geographic Region } \\
$\begin{array}{c}\text { New } \\
\text { England }\end{array}$ & $\begin{array}{c}\text { Mid } \\
\text { Atlantic }\end{array}$ & $\begin{array}{c}\text { East } \\
\text { North } \\
\text { Central }\end{array}$ & $\begin{array}{c}\text { West } \\
\text { North } \\
\text { Central }\end{array}$ & $\begin{array}{c}\text { South } \\
\text { Atlantic }\end{array}$ & $\begin{array}{c}\text { East } \\
\text { South } \\
\text { Central }\end{array}$ & $\begin{array}{c}\text { West } \\
\text { South } \\
\text { Central }\end{array}$ & Mountain & Pacific \\
QNA & QNA & QNA & QNA & QNA & QNA & QNA & QNA & QNA \\
QNA & QNA & QNA & QNA & QNA & QNA & QNA & QNA & QNA \\
$\ddagger$ & $\ddagger$ & $\ddagger$ & $\ddagger$ & $\ddagger$ & $\ddagger$ & $\ddagger$ & $\ddagger$ & $\ddagger$ \\
$\ddagger$ & $\ddagger$ & $\ddagger$ & $\ddagger$ & $\ddagger$ & $\ddagger$ & $\ddagger$ & $\ddagger$ & $\ddagger$ \\
$\ddagger$ & 7.5 & 7.6 & 7.9 & 7.5 & $\ddagger$ & 7.7 & 7.5 & 7.5 \\
$\ddagger$ & 7.4 & 7.4 & 7.6 & 7.4 & 7.5 & 7.3 & 7.3 & 7.4 \\
$\ddagger$ & 7.2 & 6.8 & $\ddagger$ & 7.3 & $\ddagger$ & 6.8 & $\ddagger$ & 7.0 \\
$\ddagger$ & 6.6 & 6.8 & 7.2 & 7.3 & $\ddagger$ & 7.4 & 7.6 & 7.0 \\
\hline
\end{tabular}

${ }^{89} \ddagger$ Reporting standard not met (too few cases); QNA: Question Not Asked

Note: Changes in sample size and stratification were implemented following November 2002 Youth Poll.

Source: Department of Defense Polls, JAMRS, 2001-2004 (Question: FAV3A). 


\section{Reserve Favorability}

JAMRS

TABLE 18-5. Youth Reserve favorability, by high school grades: $2001-2004^{90}$

\begin{tabular}{|c|c|c|c|c|c|c|c|}
\hline \multicolumn{8}{|c|}{ Male and Female (age 16-21) } \\
\hline \multirow{2}{*}{$\begin{array}{l}\text { (mean) } \\
\text { Year }\end{array}$} & \multicolumn{7}{|c|}{ High School Grades } \\
\hline & $\begin{array}{l}\text { Mostly } \\
\text { A's }\end{array}$ & $\begin{array}{l}\text { Mostly A's } \\
\text { \& B's }\end{array}$ & $\begin{array}{l}\text { Mostly } \\
\text { B's }\end{array}$ & $\begin{array}{l}\text { Mostly B's } \\
\text { \& C's }\end{array}$ & $\begin{array}{l}\text { Mostly } \\
\text { C's }\end{array}$ & $\begin{array}{l}\text { Mostly C's } \\
\text { \& D's }\end{array}$ & $\begin{array}{l}\text { Mostly D's } \\
\text { and Lower }\end{array}$ \\
\hline Youth Poll 1 (Apr 01) & QNA & QNA & QNA & QNA & QNA & QNA & QNA \\
\hline Youth Poll 2 (Aug 01) & QNA & QNA & QNA & QNA & QNA & QNA & QNA \\
\hline Youth Poll 3 (Nov 01) & 8.1 & 8.2 & 8.1 & 8.2 & $\ddagger$ & $\ddagger$ & $\ddagger$ \\
\hline Youth Poll 4 (Nov 02) & 7.2 & 7.2 & 7.2 & 6.9 & 6.6 & $\ddagger$ & $\ddagger$ \\
\hline Youth Poll 5 (June 03) & 7.5 & 7.5 & 7.2 & 7.3 & 6.9 & 7.1 & $\ddagger$ \\
\hline Youth Poll 6 (Nov 03) & 7.3 & 7.4 & 7.1 & 7.1 & 6.5 & 7.2 & $\ddagger$ \\
\hline Youth Poll 7 (May 04) & 7.1 & 7.2 & 6.8 & 6.8 & 6.3 & 6.8 & $\ddagger$ \\
\hline Youth Poll 8 (Nov 04) & 7.3 & 7.0 & 6.8 & 6.8 & 6.5 & 7.0 & $\ddagger$ \\
\hline \multicolumn{8}{|l|}{ Male (age 16-21) } \\
\hline (mean) & \multicolumn{7}{|c|}{ High School Grades } \\
\hline Year & $\begin{array}{l}\text { Mostly } \\
\text { A's }\end{array}$ & $\begin{array}{l}\text { Mostly A's } \\
\text { \& B's }\end{array}$ & $\begin{array}{l}\text { Mostly } \\
\text { B's }\end{array}$ & $\begin{array}{l}\text { Mostly B's } \\
\text { \& C's }\end{array}$ & $\begin{array}{l}\text { Mostly } \\
\text { C's }\end{array}$ & $\begin{array}{l}\text { Mostly C's } \\
\text { \& D's }\end{array}$ & $\begin{array}{l}\text { Mostly D's } \\
\text { and Lower }\end{array}$ \\
\hline Youth Poll 1 (Apr 01) & QNA & QNA & QNA & QNA & QNA & QNA & QNA \\
\hline Youth Poll 2 (Aug 01) & QNA & QNA & QNA & QNA & QNA & QNA & QNA \\
\hline Youth Poll 3 (Nov 01) & 8.0 & 8.0 & 7.9 & 8.0 & $\ddagger$ & $\ddagger$ & $\ddagger$ \\
\hline Youth Poll 4 (Nov 02) & $\ddagger$ & 6.9 & 6.9 & 6.7 & $\ddagger$ & $\ddagger$ & $\ddagger$ \\
\hline Youth Poll 5 (June 03) & 7.4 & 7.2 & 7.0 & 7.2 & 6.6 & 6.6 & $\ddagger$ \\
\hline Youth Poll 6 (Nov 03) & 6.9 & 7.3 & 7.1 & 6.9 & 6.5 & 7.2 & $\ddagger$ \\
\hline Youth Poll 7 (May 04) & 7.0 & 7.2 & 6.6 & 6.9 & $\ddagger$ & 6.9 & $\ddagger$ \\
\hline Youth Poll 8 (Nov 04) & 7.2 & 6.9 & 6.8 & 6.7 & 6.3 & $\ddagger$ & $\ddagger$ \\
\hline
\end{tabular}

Females (age 16-21)

(mean)

High School Grades

\begin{tabular}{lccccccc} 
& $\begin{array}{c}\text { Mostly } \\
\text { A's }\end{array}$ & $\begin{array}{c}\text { Mostly A's } \\
\text { \& B's }\end{array}$ & $\begin{array}{c}\text { Mostly } \\
\text { B's }\end{array}$ & $\begin{array}{c}\text { Mostly B's } \\
\text { \& C's }\end{array}$ & $\begin{array}{c}\text { Mostly } \\
\text { C's }\end{array}$ & $\begin{array}{c}\text { Mostly C's } \\
\text { \& D's }\end{array}$ & $\begin{array}{c}\text { Mostly D's } \\
\text { and Lower }\end{array}$ \\
\hline Youth Poll 1 (Apr 01) & QNA & QNA & QNA & QNA & QNA & QNA & QNA \\
Youth Poll 2 (Aug 01) & QNA & QNA & QNA & QNA & QNA & QNA & QNA \\
Youth Poll 3 (Nov 01) & 8.1 & 8.4 & 8.3 & 8.5 & $\ddagger$ & $\ddagger$ & $\ddagger$ \\
Youth Poll 4 (Nov 02) & 7.5 & 7.4 & 7.5 & 7.2 & $\ddagger$ & $\ddagger$ & $\ddagger$ \\
Youth Poll 5 (June 03) & 7.5 & 7.7 & 7.4 & 7.5 & $\ddagger$ & $\ddagger$ & $\ddagger$ \\
Youth Poll 6 (Nov 03) & 7.6 & 7.5 & 7.0 & 7.4 & $\ddagger$ & $\ddagger$ & $\ddagger$ \\
Youth Poll 7 (May 04) & 7.2 & 7.2 & 6.9 & 6.8 & $\ddagger$ & $\ddagger$ & $\ddagger$ \\
Youth Poll 8 (Nov 04) & 7.4 & 7.1 & 6.9 & 7.0 & $\ddagger$ & $\ddagger$ & $\ddagger$
\end{tabular}

${ }^{90}$ ‡Reporting standard not met (too few cases); QNA: Question Not Asked

Note: Changes in sample size and stratification were implemented following November 2002 Youth Poll.

Source: Department of Defense Polls, JAMRS, 2001-2004 (Question: FAV3A). 


\section{National Guard Favorability}

TABLE 19-1. Youth National Guard favorability: $2001-2004^{91}$

\begin{tabular}{lc}
$\begin{array}{l}\text { Male and Female (age 16-21) } \\
\text { (mean) }\end{array}$ & \\
\cline { 2 - 2 } Year & Mean \\
\hline Youth Poll 1 (Apr 01) & QNA \\
Youth Poll 2 (Aug 01) & QNA \\
Youth Poll 3 (Nov 01) & 8.2 \\
Youth Poll 4 (Nov 02) & 7.1 \\
Youth Poll 5 (June 03) & 7.4 \\
Youth Poll 6 (Nov 03) & 7.1 \\
Youth Poll 7 (May 04) & 7.0 \\
Youth Poll 8 (Nov 04) & 6.9 \\
\hline
\end{tabular}

\begin{tabular}{lc}
$\begin{array}{l}\text { Male (age 16-21) } \\
\text { (mean) }\end{array}$ \\
\cline { 2 - 2 } Year
\end{tabular}

\begin{tabular}{lc}
$\begin{array}{l}\text { Female (age 16-21) } \\
\text { (mean) }\end{array}$ & \\
\cline { 2 - 2 } Year & Mean \\
\hline Youth Poll 1 (Apr 01) & QNA \\
Youth Poll 2 (Aug 01) & QNA \\
Youth Poll 3 (Nov 01) & 8.4 \\
Youth Poll 4 (Nov 02) & 7.4 \\
Youth Poll 5 (June 03) & 7.6 \\
Youth Poll 6 (Nov 03) & 7.4 \\
Youth Poll 7 (May 04) & 7.1 \\
Youth Poll 8 (Nov 04) & 7.1 \\
\hline
\end{tabular}

${ }^{91} \ddagger$ Reporting standard not met (too few cases); QNA: Question Not Asked Note: Changes in sample size and stratification were implemented following November 2002 Youth Poll. Source: Department of Defense Polls, JAMRS, 2001-2004 (Question: FAV3B). 


\section{National Guard Favorability}

TABLE 19-2. Youth National Guard favorability, by race/ethnicity: $2001-2004^{92}$

\begin{tabular}{lccc} 
Male and Female (age 16-21) & \multicolumn{3}{c}{ Race/ethnicity } \\
\cline { 2 - 4 } (mean) & White & Black & Hispanic \\
\cline { 2 - 4 } Year & QNA & QNA & QNA \\
\hline Youth Poll 1 (Apr 01) & QNA & QNA & QNA \\
Youth Poll 2 (Aug 01) & 8.2 & 8.3 & 8.2 \\
Youth Poll 3 (Nov 01) & 7.2 & 6.9 & 6.9 \\
Youth Poll 4 (Nov 02) & 7.5 & 6.7 & 7.4 \\
Youth Poll 5 (June 03) & 7.3 & 6.7 & 7.1 \\
Youth Poll 6 (Nov 03) & 7.1 & 6.4 & 6.9 \\
Youth Poll 7 (May 04) & 7.2 & 5.9 & 6.9 \\
Youth Poll 8 (Nov 04) & & & \\
\hline
\end{tabular}

\begin{tabular}{lccc} 
Male (age 16-21) & \multicolumn{3}{c}{ Racelethnicity } \\
\cline { 2 - 4 } (mean) & White & Black & Hispanic \\
\cline { 2 - 4 } Year & QNA & QNA & QNA \\
\hline Youth Poll 1 (Apr 01) & QNA & QNA & QNA \\
Youth Poll 2 (Aug 01) & 8.0 & 8.3 & 7.9 \\
Youth Poll 3 (Nov 01) & 6.9 & 7.0 & 6.6 \\
Youth Poll 4 (Nov 02) & 7.2 & 6.6 & 7.1 \\
Youth Poll 5 (June 03) & 7.0 & 6.7 & 6.9 \\
Youth Poll 6 (Nov 03) & 7.0 & 6.4 & 6.7 \\
Youth Poll 7 (May 04) & 7.1 & 6.0 & 6.6 \\
Youth Poll 8 (Nov 04) & & &
\end{tabular}

\begin{tabular}{lccc} 
Female (age 16-21) & \multicolumn{3}{c}{ Race/ethnicity } \\
\cline { 2 - 4 } (mean) & White & Black & Hispanic \\
\cline { 2 - 4 } Year & QNA & QNA & QNA \\
\hline Youth Poll 1 (Apr 01) & QNA & QNA & QNA \\
Youth Poll 2 (Aug 01) & 8.5 & 8.4 & 8.4 \\
Youth Poll 3 (Nov 01) & 7.6 & 6.9 & 7.2 \\
Youth Poll 4 (Nov 02) & 7.9 & 6.8 & 7.8 \\
Youth Poll 5 (June 03) & 7.6 & 6.6 & 7.3 \\
Youth Poll 6 (Nov 03) & 7.3 & 6.3 & 7.1 \\
Youth Poll 7 (May 04) & 7.4 & 5.9 & 7.2 \\
Youth Poll 8 (Nov 04) & & & \\
\hline
\end{tabular}

${ }^{92}$ Due to relatively small sample sizes, American Indian, Alaska Native, Asian, and Pacific Islander are included in the total but are not shown separately. Black includes African American, and Hispanic includes Latino. Race categories exclude Hispanic origin unless specified otherwise.

†Reporting standard not met (too few cases); QNA: Question Not Asked

Note: Changes in sample size and stratification were implemented following November 2002 Youth Poll.

Source: Department of Defense Polls, JAMRS, 2001-2004 (Question: FAV3B). 


\section{National Guard Favorability}

JAMRS

TABLE 19-3. Youth National Guard favorability, by age: $2001-2004^{93}$

\begin{tabular}{|c|c|c|c|c|c|c|}
\hline \multirow{3}{*}{$\begin{array}{l}\text { Male and Female (age 16-21) } \\
\text { (mean) } \\
\text { Year }\end{array}$} & & & & & & \\
\hline & \multicolumn{6}{|c|}{ Age } \\
\hline & 16 & 17 & 18 & 19 & 20 & 21 \\
\hline Youth Poll 1 (Apr 01) & QNA & QNA & QNA & QNA & QNA & QNA \\
\hline Youth Poll 2 (Aug 01) & QNA & QNA & QNA & QNA & QNA & QNA \\
\hline Youth Poll 3 (Nov 01) & 8.2 & 8.1 & 8.2 & 8.4 & 8.3 & 8.1 \\
\hline Youth Poll 4 (Nov 02) & 7.2 & 7.0 & 6.9 & 7.2 & 6.8 & 7.6 \\
\hline Youth Poll 5 (June 03) & 7.6 & 7.3 & 7.3 & 7.4 & 7.3 & 7.4 \\
\hline Youth Poll 6 (Nov 03) & 7.1 & 7.1 & 7.2 & 7.2 & 7.3 & 7.0 \\
\hline Youth Poll 7 (May 04) & 7.1 & 7.0 & 7.0 & 7.1 & 6.9 & 6.8 \\
\hline Youth Poll 8 (Nov 04) & 7.1 & 6.9 & 7.0 & 6.7 & 6.9 & 7.1 \\
\hline
\end{tabular}

\begin{tabular}{lcccccc} 
Male (age 16-21) & \multicolumn{7}{c}{ Age } \\
\cline { 2 - 7 } (mean) & $\mathbf{1 6}$ & $\mathbf{1 7}$ & $\mathbf{1 8}$ & $\mathbf{1 9}$ & $\mathbf{2 0}$ & $\mathbf{2 1}$ \\
\cline { 2 - 7 } Year & QNA & QNA & QNA & QNA & QNA & QNA \\
\hline Youth Poll 1 (Apr 01) & QNA & QNA & QNA & QNA & QNA & QNA \\
Youth Poll 2 (Aug 01) & 8.0 & 8.1 & 7.9 & 8.2 & 7.9 & 8.0 \\
Youth Poll 3 (Nov 01) & 7.1 & 6.7 & 6.9 & 6.4 & 6.5 & 7.2 \\
Youth Poll 4 (Nov 02) & 7.4 & 6.9 & 7.0 & 7.2 & 6.8 & 7.3 \\
Youth Poll 5 (June 03) & 6.8 & 6.8 & 6.9 & 7.0 & 7.1 & 6.9 \\
Youth Poll 6 (Nov 03) & 6.9 & 7.0 & 6.7 & 7.0 & 6.6 & 7.0 \\
Youth Poll 7 (May 04) & 7.0 & 6.7 & 6.9 & 6.5 & 6.9 & 7.0 \\
Youth Poll 8 (Nov 04) & & & & & 6.9 & \\
\hline
\end{tabular}

\section{Female (age 16-21)}

(mean)

Year

Youth Poll 1 (Apr 01)

Youth Poll 2 (Aug 01)

Youth Poll 3 (Nov 01)

Youth Poll 4 (Nov 02)

Youth Poll 5 (June 03)

Youth Poll 6 (Nov 03)

Youth Poll 7 (May 04)

\begin{tabular}{cccccc}
\hline \multicolumn{7}{c}{ Age } \\
\hline $\mathbf{1 6}$ & $\mathbf{1 7}$ & $\mathbf{1 8}$ & $\mathbf{1 9}$ & $\mathbf{2 0}$ & $\mathbf{2 1}$ \\
QNA & QNA & QNA & QNA & QNA & QNA \\
QNA & QNA & QNA & QNA & QNA & QNA \\
8.4 & 8.1 & 8.4 & 8.6 & 8.7 & 8.3 \\
7.3 & 7.3 & 6.8 & 8.0 & 7.1 & 7.8 \\
7.8 & 7.8 & 7.6 & 7.6 & 7.8 & 7.4 \\
7.4 & 7.3 & 7.4 & 7.5 & 7.5 & 7.1 \\
7.4 & 6.9 & 7.2 & 7.2 & 7.2 & 6.6 \\
7.2 & 7.0 & 7.1 & 6.9 & 6.9 & 7.2 \\
\hline
\end{tabular}

Youth Poll 8 (Nov 04)

${ }^{93}$ ‡Reporting standard not met (too few cases); QNA: Question Not Asked

Note: Changes in sample size and stratification were implemented following November 2002 Youth Poll.

Source: Department of Defense Polls, JAMRS, 2001-2004 (Question: FAV3B).

Page A-93 DoD May 2004 Youth Poll 


\section{National Guard Favorability}

JAMRS

TABLE 19-4. Youth National Guard favorability, by geographic region: $2001-2004^{94}$

\section{Male and Female (age 16-21)}

(mean)

Year

Youth Poll 1 (Apr 01)

Youth Poll 2 (Aug 01)

Youth Poll 3 (Nov 01)

Youth Poll 4 (Nov 02)

Youth Poll 5 (June 03)

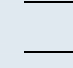

Youth Poll 6 (Nov 03)

Youth Poll 7 (May 04)

New
England

Youth Poll 8 (Nov 04)

$\begin{array}{cc}\text { QNA } & \text { QNA } \\ \text { QNA } & \text { QNA } \\ \ddagger & \ddagger \\ \ddagger & \ddagger \\ 7.1 & 7.3 \\ 7.1 & 7.1 \\ 7.0 & 7.0 \\ 6.4 & 6.6\end{array}$

Mid
Atlantic

East
North
Central

QNA

QNA

\section{Geographic Region}

\begin{tabular}{cccccc}
$\begin{array}{c}\text { West } \\
\text { North } \\
\text { Central }\end{array}$ & $\begin{array}{c}\text { South } \\
\text { Atlantic }\end{array}$ & $\begin{array}{c}\text { East } \\
\text { South } \\
\text { Central }\end{array}$ & $\begin{array}{c}\text { West } \\
\text { South } \\
\text { Central }\end{array}$ & Mountain & Pacific \\
QNA & QNA & QNA & QNA & QNA & QNA \\
QNA & QNA & QNA & QNA & QNA & QNA \\
$\ddagger$ & $\ddagger$ & $\ddagger$ & $\ddagger$ & $\ddagger$ & $\ddagger$ \\
$\ddagger$ & $\ddagger$ & $\ddagger$ & $\ddagger$ & $\ddagger$ & $\ddagger$ \\
7.5 & 7.4 & 7.4 & 7.4 & 7.2 & 7.3 \\
7.5 & 7.2 & 7.4 & 7.0 & 7.2 & 7.0 \\
7.0 & 7.2 & 7.4 & 6.9 & 6.9 & 6.7 \\
7.2 & 7.1 & 7.2 & 7.1 & 7.4 & 6.7 \\
\hline
\end{tabular}

Male (age 16-21)

(mean)

\section{Geographic Region}

\begin{tabular}{lccccccccc}
\cline { 2 - 8 } Year & $\begin{array}{c}\text { New } \\
\text { England }\end{array}$ & $\begin{array}{c}\text { Mid } \\
\text { Atlantic }\end{array}$ & $\begin{array}{c}\text { East } \\
\text { North } \\
\text { Central }\end{array}$ & $\begin{array}{c}\text { West } \\
\text { North } \\
\text { Central }\end{array}$ & $\begin{array}{c}\text { South } \\
\text { Atlantic }\end{array}$ & $\begin{array}{c}\text { East } \\
\text { South } \\
\text { Central }\end{array}$ & $\begin{array}{c}\text { West } \\
\text { South } \\
\text { Central }\end{array}$ & Mountain & Pacific \\
\hline Youth Poll 1 (Apr 01) & QNA & QNA & QNA & QNA & QNA & QNA & QNA & QNA & QNA \\
Youth Poll 2 (Aug 01) & QNA & QNA & QNA & QNA & QNA & QNA & QNA & QNA & QNA \\
Youth Poll 3 (Nov 01) & $\ddagger$ & $\ddagger$ & $\ddagger$ & $\ddagger$ & $\ddagger$ & $\ddagger$ & $\ddagger$ & $\ddagger$ & $\ddagger$ \\
Youth Poll 4 (Nov 02) & $\ddagger$ & $\ddagger$ & $\ddagger$ & $\ddagger$ & $\ddagger$ & $\ddagger$ & $\ddagger$ & $\ddagger$ & $\ddagger$ \\
Youth Poll 5 (June 03) & $\ddagger$ & 7.3 & 7.3 & 7.0 & 7.2 & $\ddagger$ & 7.1 & 6.8 & 7.0 \\
Youth Poll 6 (Nov 03) & $\ddagger$ & 6.9 & 6.7 & 7.3 & 6.9 & $\ddagger$ & 6.8 & $\ddagger$ & 6.8 \\
Youth Poll 7 (May 04) & $\ddagger$ & 7.0 & 7.0 & $\ddagger$ & 7.0 & $\ddagger$ & 6.9 & $\ddagger$ & 6.6 \\
Youth Poll 8 (Nov 04) & $\ddagger$ & 6.6 & 6.9 & 7.2 & 7.0 & $\ddagger$ & 6.8 & 7.1 & 6.6 \\
\hline
\end{tabular}

Female (age 16-21)

(mean)

Year

Youth Poll 1 (Apr 01)

Youth Poll 2 (Aug 01)

Youth Poll 3 (Nov 01)

Youth Poll 4 (Nov 02)

Youth Poll 5 (June 03)

Youth Poll 6 (Nov 03)

Youth Poll 7 (May 04)

Youth Poll 8 (Nov 04)

\begin{tabular}{ccccccccc}
\hline \multicolumn{10}{c}{ Geographic Region } \\
$\begin{array}{c}\text { New } \\
\text { England }\end{array}$ & $\begin{array}{c}\text { Mid } \\
\text { Atlantic }\end{array}$ & $\begin{array}{c}\text { East } \\
\text { North } \\
\text { Central }\end{array}$ & $\begin{array}{c}\text { West } \\
\text { North } \\
\text { Central }\end{array}$ & $\begin{array}{c}\text { South } \\
\text { Atlantic }\end{array}$ & $\begin{array}{c}\text { East } \\
\text { South } \\
\text { Central }\end{array}$ & $\begin{array}{c}\text { West } \\
\text { South } \\
\text { Central }\end{array}$ & Mountain & Pacific \\
QNA & QNA & QNA & QNA & QNA & QNA & QNA & QNA & QNA \\
QNA & QNA & QNA & QNA & QNA & QNA & QNA & QNA & QNA \\
$\ddagger$ & $\ddagger$ & $\ddagger$ & $\ddagger$ & $\ddagger$ & $\ddagger$ & $\ddagger$ & $\ddagger$ & $\ddagger$ \\
$\ddagger$ & $\ddagger$ & $\ddagger$ & $\ddagger$ & $\ddagger$ & $\ddagger$ & $\ddagger$ & $\ddagger$ & $\ddagger$ \\
$\ddagger$ & 7.4 & 7.7 & 8.0 & 7.6 & $\ddagger$ & 7.6 & 7.7 & 7.6 \\
$\ddagger$ & 7.3 & 7.5 & 7.7 & 7.4 & 7.6 & 7.2 & 7.3 & 7.3 \\
$\ddagger$ & 7.0 & 6.9 & $\ddagger$ & 7.4 & $\ddagger$ & 6.9 & $\ddagger$ & 6.9 \\
$\ddagger$ & 6.5 & 6.8 & 7.3 & 7.2 & $\ddagger$ & 7.3 & 7.7 & 7.0 \\
\hline
\end{tabular}

${ }^{94}$ ‡Reporting standard not met (too few cases); QNA: Question Not Asked

Note: Changes in sample size and stratification were implemented following November 2002 Youth Poll.

Source: Department of Defense Polls, JAMRS, 2001-2004 (Question: FAV3B). 


\section{National Guard Favorability}

JAMRS

TABLE 19-5. Youth National Guard favorability, by high school grades: $2001-2004^{95}$

\begin{tabular}{|c|c|c|c|c|c|c|c|}
\hline \multicolumn{8}{|c|}{ Male and Female (age 16-21) } \\
\hline \multirow{2}{*}{$\begin{array}{l}\text { (mean) } \\
\text { Year }\end{array}$} & \multicolumn{7}{|c|}{ High School Grades } \\
\hline & $\begin{array}{l}\text { Mostly } \\
\text { A's }\end{array}$ & $\begin{array}{l}\text { Mostly A's } \\
\& \text { B's }\end{array}$ & $\begin{array}{l}\text { Mostly } \\
\text { B's }\end{array}$ & $\begin{array}{l}\text { Mostly B's } \\
\text { \& C's }\end{array}$ & $\begin{array}{l}\text { Mostly } \\
\text { C's }\end{array}$ & $\begin{array}{l}\text { Mostly C's } \\
\text { \& D's }\end{array}$ & $\begin{array}{l}\text { Mostly D's } \\
\text { and Lower }\end{array}$ \\
\hline Youth Poll 1 (Apr 01) & QNA & QNA & QNA & QNA & QNA & QNA & QNA \\
\hline Youth Poll 2 (Aug 01) & QNA & QNA & QNA & QNA & QNA & QNA & QNA \\
\hline Youth Poll 3 (Nov 01) & 8.0 & 8.3 & 8.2 & 8.2 & $\ddagger$ & $\ddagger$ & $\ddagger$ \\
\hline Youth Poll 4 (Nov 02) & 7.3 & 7.3 & 7.2 & 6.9 & 7.0 & $\ddagger$ & $\ddagger$ \\
\hline Youth Poll 5 (June 03) & 7.5 & 7.6 & 7.2 & 7.3 & 7.0 & 7.0 & $\ddagger$ \\
\hline Youth Poll 6 (Nov 03) & 7.4 & 7.3 & 7.1 & 7.1 & 6.5 & 7.0 & $\ddagger$ \\
\hline Youth Poll 7 (May 04) & 7.2 & 7.2 & 6.7 & 6.9 & 6.4 & 6.8 & $\ddagger$ \\
\hline Youth Poll 8 (Nov 04) & 7.3 & 7.0 & 6.8 & 6.9 & 6.7 & 6.3 & $\ddagger$ \\
\hline \multicolumn{8}{|l|}{ Male (age 16-21) } \\
\hline (mean) & \multicolumn{7}{|c|}{ High School Grades } \\
\hline Year & $\begin{array}{l}\text { Mostly } \\
\text { A's }\end{array}$ & $\begin{array}{l}\text { Mostly A's } \\
\text { \& B's }\end{array}$ & $\begin{array}{l}\text { Mostly } \\
\text { B's }\end{array}$ & $\begin{array}{l}\text { Mostly B's } \\
\text { \& C'S }\end{array}$ & $\begin{array}{l}\text { Mostly } \\
\text { C's }\end{array}$ & $\begin{array}{l}\text { Mostly C's } \\
\text { \& D's }\end{array}$ & $\begin{array}{l}\text { Mostly D's } \\
\text { and Lower }\end{array}$ \\
\hline Youth Poll 1 (Apr 01) & QNA & QNA & QNA & QNA & QNA & QNA & QNA \\
\hline Youth Poll 2 (Aug 01) & QNA & QNA & QNA & QNA & QNA & QNA & QNA \\
\hline Youth Poll 3 (Nov 01) & 8.1 & 8.0 & 7.9 & 7.9 & $\ddagger$ & $\ddagger$ & $\ddagger$ \\
\hline Youth Poll 4 (Nov 02) & $\ddagger$ & 7.0 & 7.0 & 6.8 & $\ddagger$ & $\ddagger$ & $\ddagger$ \\
\hline Youth Poll 5 (June 03) & 7.4 & 7.3 & 6.9 & 7.1 & 6.8 & 6.4 & $\ddagger$ \\
\hline Youth Poll 6 (Nov 03) & 7.0 & 7.2 & 7.0 & 6.7 & 6.4 & 6.9 & $\ddagger$ \\
\hline Youth Poll 7 (May 04) & 7.0 & 7.2 & 6.6 & 6.9 & $\ddagger$ & 6.8 & $\ddagger$ \\
\hline Youth Poll 8 (Nov 04) & 7.1 & 6.9 & 6.7 & 6.9 & 6.6 & $\ddagger$ & $\ddagger$ \\
\hline
\end{tabular}

\begin{tabular}{lccccccc} 
Females (age 16-21) & \multicolumn{7}{c}{ High School Grades } \\
\cline { 2 - 7 } (mean) & $\begin{array}{c}\text { Mostly } \\
\text { A's }\end{array}$ & $\begin{array}{c}\text { Mostly A's } \\
\text { \& B's }\end{array}$ & $\begin{array}{c}\text { Mostly } \\
\text { B's }\end{array}$ & $\begin{array}{c}\text { Mostly B's } \\
\text { \& C's }\end{array}$ & $\begin{array}{c}\text { Mostly } \\
\text { C's }\end{array}$ & $\begin{array}{c}\text { Mostly C's } \\
\text { \& D's }\end{array}$ & $\begin{array}{c}\text { Mostly D's } \\
\text { and Lower }\end{array}$ \\
\cline { 2 - 7 } & QNA & QNA & QNA & QNA & QNA & QNA & QNA \\
\hline Youth Poll 1 (Apr 01) & QNA & QNA & QNA & QNA & QNA & QNA & QNA \\
Youth Poll 2 (Aug 01) & 8.0 & 8.5 & 8.5 & 8.6 & $\ddagger$ & $\ddagger$ & $\ddagger$ \\
Youth Poll 3 (Nov 01) & 7.7 & 7.5 & 7.3 & 7.1 & $\ddagger$ & $\ddagger$ & $\ddagger$ \\
Youth Poll 4 (Nov 02) & 7.5 & 7.8 & 7.4 & 7.6 & $\ddagger$ & $\ddagger$ & $\ddagger$ \\
Youth Poll 5 (June 03) & 7.7 & 7.5 & 7.1 & 7.4 & $\ddagger$ & $\ddagger$ & $\ddagger$ \\
Youth Poll 6 (Nov 03) & 7.3 & 7.2 & 6.9 & 6.9 & $\ddagger$ & $\ddagger$ & $\ddagger$ \\
Youth Poll 7 (May 04) & 7.5 & 7.1 & 6.9 & 7.0 & $\ddagger$ & $\ddagger$ & $\ddagger$ \\
Youth Poll 8 (Nov 04) & & & & & & $\ddagger$ \\
\hline
\end{tabular}

${ }^{95}$ ‡Reporting standard not met (too few cases); QNA: Question Not Asked

Note: Changes in sample size and stratification were implemented following November 2002 Youth Poll.

Source: Department of Defense Polls, JAMRS, 2001-2004 (Question: FAV3B). 


\section{U.S. Military Knowledge}

TABLE 20-1. Youth U.S. Military knowledge: $2001-2004^{96}$

\begin{tabular}{lc}
$\begin{array}{l}\text { Male and Female (age 16-21) } \\
\text { (mean) }\end{array}$ & \\
\cline { 2 - 2 } Year & Mean \\
\hline Youth Poll 1 (Apr 01) & QNA \\
Youth Poll 2 (Aug 01) & QNA \\
Youth Poll 3 (Nov 01) & 5.8 \\
Youth Poll 4 (Nov 02) & 5.2 \\
Youth Poll 5 (June 03) & 5.6 \\
Youth Poll 6 (Nov 03) & 5.4 \\
Youth Poll 7 (May 04) & 5.2 \\
Youth Poll 8 (Nov 04) & 5.3 \\
\hline
\end{tabular}

\begin{tabular}{lc}
$\begin{array}{l}\text { Male (age 16-21) } \\
\text { (mean) }\end{array}$ \\
\cline { 2 - 2 } Year & Mean \\
\hline Youth Poll 1 (Apr 01) & QNA \\
Youth Poll 2 (Aug 01) & QNA \\
Youth Poll 3 (Nov 01) & 6.1 \\
Youth Poll 4 (Nov 02) & 5.5 \\
Youth Poll 5 (June 03) & 5.8 \\
Youth Poll 6 (Nov 03) & 5.7 \\
Youth Poll 7 (May 04) & 5.6 \\
Youth Poll 8 (Nov 04) & 5.6 \\
\hline
\end{tabular}

\begin{tabular}{lc}
$\begin{array}{l}\text { Female (age 16-21) } \\
\text { (mean) }\end{array}$ & \\
\cline { 2 - 2 } Year & Mean \\
\hline Youth Poll 1 (Apr 01) & QNA \\
Youth Poll 2 (Aug 01) & QNA \\
Youth Poll 3 (Nov 01) & 5.5 \\
Youth Poll 4 (Nov 02) & 4.9 \\
Youth Poll 5 (June 03) & 5.3 \\
Youth Poll 6 (Nov 03) & 5.0 \\
Youth Poll 7 (May 04) & 4.8 \\
Youth Poll 8 (Nov 04) & 5.0 \\
\hline
\end{tabular}

${ }^{96}$ ‡Reporting standard not met (too few cases); QNA: Question Not Asked Note: Changes in sample size and stratification were implemented following November 2002 Youth Poll. Source: Department of Defense Polls, JAMRS, 2001-2004 (Question: KW2). 


\section{U.S. Military Knowledge}

TABLE 20-2. Youth U.S. Military knowledge, by race/ethnicity: $2001-2004^{97}$

\begin{tabular}{lccc} 
Male and Female (age 16-21) & \multicolumn{3}{c}{ Racelethnicity } \\
\cline { 2 - 4 } (mean) & White & Black & Hispanic \\
\cline { 2 - 4 } Year & QNA & QNA & QNA \\
\hline Youth Poll 1 (Apr 01) & QNA & QNA & QNA \\
Youth Poll 2 (Aug 01) & 5.7 & 6.0 & 5.8 \\
Youth Poll 3 (Nov 01) & 5.1 & 5.1 & 5.5 \\
Youth Poll 4 (Nov 02) & 5.6 & 5.5 & 5.5 \\
Youth Poll 5 (June 03) & 5.4 & 5.6 & 5.3 \\
Youth Poll 6 (Nov 03) & 5.3 & 4.8 & 5.3 \\
Youth Poll 7 (May 04) & 5.4 & 5.0 & 5.3 \\
Youth Poll 8 (Nov 04) & & & \\
\hline
\end{tabular}

\begin{tabular}{lccc} 
Male (age 16-21) & \multicolumn{3}{c}{ Racelethnicity } \\
\cline { 2 - 4 } (mean) & White & Black & Hispanic \\
\cline { 2 - 4 } Year & QNA & QNA & QNA \\
\hline Youth Poll 1 (Apr 01) & QNA & QNA & QNA \\
Youth Poll 2 (Aug 01) & 6.1 & 6.3 & 6.0 \\
Youth Poll 3 (Nov 01) & 5.5 & 5.3 & 5.6 \\
Youth Poll 4 (Nov 02) & 6.0 & 5.4 & 5.6 \\
Youth Poll 5 (June 03) & 5.8 & 5.7 & 5.6 \\
Youth Poll 6 (Nov 03) & 5.7 & 5.0 & 5.6 \\
Youth Poll 7 (May 04) & 5.8 & 4.9 & 5.4 \\
Youth Poll 8 (Nov 04) & & &
\end{tabular}

\begin{tabular}{lccc} 
Female (age 16-21) & \multicolumn{3}{c}{ Race/ethnicity } \\
\cline { 2 - 4 } (mean) & White & Black & Hispanic \\
\cline { 2 - 4 } Year & QNA & QNA & QNA \\
\hline Youth Poll 1 (Apr 01) & QNA & QNA & QNA \\
Youth Poll 2 (Aug 01) & 5.4 & 5.8 & 5.6 \\
Youth Poll 3 (Nov 01) & 4.8 & 4.8 & 5.3 \\
Youth Poll 4 (Nov 02) & 5.2 & 5.6 & 5.4 \\
Youth Poll 5 (June 03) & 4.9 & 5.5 & 5.0 \\
Youth Poll 6 (Nov 03) & 4.8 & 4.6 & 5.1 \\
Youth Poll 7 (May 04) & 4.9 & 5.0 & 5.1 \\
Youth Poll 8 (Nov 04) & & & \\
\hline
\end{tabular}

${ }^{97}$ Due to relatively small sample sizes, American Indian, Alaska Native, Asian, and Pacific Islander are included in the total but are not shown separately. Black includes African American, and Hispanic includes Latino. Race categories exclude Hispanic origin unless specified otherwise.

†Reporting standard not met (too few cases); QNA: Question Not Asked

Note: Changes in sample size and stratification were implemented following November 2002 Youth Poll.

Source: Department of Defense Polls, JAMRS, 2001-2004 (Question: KW2). 


\section{U.S. Military Knowledge}

JAMRS

TABLE 20-3. Youth U.S. Military knowledge, by age: $2001-2004^{98}$

\begin{tabular}{|c|c|c|c|c|c|c|}
\hline \multirow{3}{*}{$\begin{array}{l}\text { Male and Female (age 16-21) } \\
\text { (mean) } \\
\text { Year }\end{array}$} & & & & & & \\
\hline & \multicolumn{6}{|c|}{ Age } \\
\hline & 16 & 17 & 18 & 19 & 20 & 21 \\
\hline Youth Poll 1 (Apr 01) & QNA & QNA & QNA & QNA & QNA & QNA \\
\hline Youth Poll 2 (Aug 01) & QNA & QNA & QNA & QNA & QNA & QNA \\
\hline Youth Poll 3 (Nov 01) & 5.6 & 5.8 & 5.6 & 5.7 & 6.1 & 5.9 \\
\hline Youth Poll 4 (Nov 02) & 5.0 & 5.1 & 5.0 & 5.2 & 5.4 & 5.4 \\
\hline Youth Poll 5 (June 03) & 5.5 & 5.6 & 5.6 & 5.4 & 5.7 & 5.6 \\
\hline Youth Poll 6 (Nov 03) & 5.2 & 5.2 & 5.2 & 5.7 & 5.5 & 5.6 \\
\hline Youth Poll 7 (May 04) & 5.1 & 5.2 & 5.3 & 5.2 & 5.3 & 5.0 \\
\hline Youth Poll 8 (Nov 04) & 5.1 & 5.2 & 5.2 & 5.3 & 5.7 & 5.3 \\
\hline
\end{tabular}

\begin{tabular}{lcccccc} 
Male (age 16-21) & \multicolumn{7}{c}{ Age } \\
\cline { 2 - 7 } (mean) & $\mathbf{1 6}$ & $\mathbf{1 7}$ & $\mathbf{1 8}$ & $\mathbf{1 9}$ & $\mathbf{2 0}$ & $\mathbf{2 1}$ \\
\cline { 2 - 7 } Year & QNA & QNA & QNA & QNA & QNA & QNA \\
\hline Youth Poll 1 (Apr 01) & QNA & QNA & QNA & QNA & QNA & QNA \\
Youth Poll 2 (Aug 01) & 6.1 & 6.2 & 5.8 & 6.0 & 6.3 & 6.2 \\
Youth Poll 3 (Nov 01) & 5.3 & 5.6 & 5.2 & 5.3 & 5.7 & 5.9 \\
Youth Poll 4 (Nov 02) & 5.7 & 5.8 & 5.8 & 5.7 & 6.1 & 5.9 \\
Youth Poll 5 (June 03) & 5.3 & 5.4 & 5.5 & 6.1 & 6.1 & 6.1 \\
Youth Poll 6 (Nov 03) & 5.5 & 5.6 & 5.6 & 5.4 & 5.8 & 5.5 \\
Youth Poll 7 (May 04) & 5.5 & 5.4 & 5.6 & 5.6 & 6.1 & 5.7 \\
Youth Poll 8 (Nov 04) & & & & & & 5.1 \\
\hline
\end{tabular}

\section{Female (age 16-21)}

(mean)

Year

Youth Poll 1 (Apr 01)

Youth Poll 2 (Aug 01)

Youth Poll 3 (Nov 01)

Youth Poll 4 (Nov 02)

Youth Poll 5 (June 03)

Youth Poll 6 (Nov 03)

Youth Poll 7 (May 04)

\begin{tabular}{cccccc}
\hline \multicolumn{7}{c}{ Age } \\
\hline $\mathbf{1 6}$ & $\mathbf{1 7}$ & $\mathbf{1 8}$ & $\mathbf{1 9}$ & $\mathbf{2 0}$ & $\mathbf{2 1}$ \\
QNA & QNA & QNA & QNA & QNA & QNA \\
QNA & QNA & QNA & QNA & QNA & QNA \\
5.2 & 5.3 & 5.4 & 5.5 & 5.8 & 5.6 \\
4.7 & 4.6 & 4.7 & 5.1 & 5.0 & 5.0 \\
5.3 & 5.3 & 5.4 & 5.0 & 5.3 & 5.4 \\
5.0 & 5.0 & 4.9 & 5.3 & 5.0 & 5.0 \\
4.7 & 4.8 & 4.9 & 5.0 & 4.8 & 4.6 \\
4.8 & 5.1 & 4.8 & 5.0 & 5.2 & 4.9 \\
\hline
\end{tabular}

Youth Poll 8 (Nov 04)

${ }^{98}$ ‡Reporting standard not met (too few cases); QNA: Question Not Asked

Note: Changes in sample size and stratification were implemented following November 2002 Youth Poll.

Source: Department of Defense Polls, JAMRS, 2001-2004 (Question: KW2).

Page A-98 DoD May 2004 Youth Poll 


\section{U.S. Military Knowledge}

JAMRS

TABLE 20-4. Youth U.S. Military knowledge, by geographic region: $2001-2004^{99}$

Male and Female (age 16-21)

(mean)

Year

Youth Poll 1 (Apr 01)

Youth Poll 2 (Aug 01)

Youth Poll 3 (Nov 01)

Youth Poll 4 (Nov 02)

Youth Poll 5 (June 03)

Youth Poll 6 (Nov 03)

Youth Poll 7 (May 04)

Mid

Youth Poll 8 (Nov 04)

$\begin{array}{cccr}\begin{array}{c}\text { New } \\ \text { England }\end{array} & \begin{array}{c}\text { Mid } \\ \text { Atlantic }\end{array} & \begin{array}{c}\text { East } \\ \text { North } \\ \text { Central }\end{array} & \begin{array}{c}\text { West } \\ \text { Nort } \\ \text { Cent }\end{array} \\ \text { QNA } & \text { QNA } & \text { QNA } & \text { QNA } \\ \text { QNA } & \text { QNA } & \text { QNA } & \text { QNA } \\ \ddagger & \ddagger & \ddagger & \ddagger \\ \ddagger & \ddagger & \ddagger & \ddagger \\ 5.7 & 5.6 & 5.3 & 5.6 \\ 5.0 & 5.5 & 5.3 & 5.5 \\ 5.1 & 5.3 & 5.0 & 5.2 \\ 4.8 & 5.1 & 5.1 & 5.3\end{array}$

Geographic Region

$\begin{array}{cccccc}\begin{array}{c}\text { West } \\ \text { North } \\ \text { Central }\end{array} & \begin{array}{c}\text { South } \\ \text { Atlantic }\end{array} & \begin{array}{c}\text { East } \\ \text { South } \\ \text { Central }\end{array} & \begin{array}{c}\text { West } \\ \text { South } \\ \text { Central }\end{array} & \text { Mountain } & \text { Pacific } \\ \text { QNA } & \text { QNA } & \text { QNA } & \text { QNA } & \text { QNA } & \text { QNA } \\ \text { QNA } & \text { QNA } & \text { QNA } & \text { QNA } & \text { QNA } & \text { QNA } \\ \ddagger & \ddagger & \ddagger & \ddagger & \ddagger & \ddagger \\ \ddagger & \ddagger & \ddagger & \ddagger & \ddagger & \ddagger \\ 5.6 & 5.6 & 5.8 & 5.7 & 5.5 & 5.5 \\ 5.5 & 5.5 & 5.6 & 5.5 & 5.4 & 5.2 \\ 5.2 & 5.5 & 4.8 & 5.1 & 5.3 & 5.1 \\ 5.3 & 5.5 & 5.9 & 5.4 & 5.3 & 5.4\end{array}$

Male (age 16-21)

(mean)

Geographic Region

Year

Youth Poll 1 (Apr 01)

Youth Poll 2 (Aug 01)

Youth Poll 3 (Nov 01)

Youth Poll 4 (Nov 02)

Youth Poll 5 (June 03)

Youth Poll 6 (Nov 03)

Youth Poll 7 (May 04)

$4.8-5.1$

\begin{tabular}{cccccc}
\hline & & \multicolumn{4}{c}{ Geographic Region } \\
$\begin{array}{c}\text { New } \\
\text { England }\end{array}$ & $\begin{array}{c}\text { Mid } \\
\text { Atlantic }\end{array}$ & $\begin{array}{c}\text { East } \\
\text { North } \\
\text { Central }\end{array}$ & $\begin{array}{c}\text { West } \\
\text { North } \\
\text { Central }\end{array}$ & $\begin{array}{c}\text { South } \\
\text { Atlantic }\end{array}$ & $\begin{array}{r}\text { East } \\
\text { So } \\
\text { Centra }\end{array}$ \\
QNA & QNA & QNA & QNA & QNA & QNA \\
QNA & QNA & QNA & QNA & QNA & QNA \\
$\ddagger$ & $\ddagger$ & $\ddagger$ & $\ddagger$ & $\ddagger$ & \\
$\ddagger$ & $\ddagger$ & $\ddagger$ & $\ddagger$ & $\ddagger$ & $\ddagger$ \\
$\ddagger$ & 5.7 & 6.1 & 5.9 & 5.7 & $\ddagger$ \\
$\ddagger$ & 5.5 & 5.9 & 6.2 & 5.7 & $\ddagger$ \\
$\ddagger$ & 5.6 & 5.5 & $\ddagger$ & 5.5 & $\ddagger$ \\
$\ddagger$ & 5.3 & 5.5 & 5.5 & 5.8 & $\ddagger$
\end{tabular}

East West

South South Mountain Pacific

Central Central

QNA QNA QNA QNA

QNA QNA

QNA

QNA

Youth Poll 8 (Nov 04)

Female (age 16-21)

(mean)

\section{Year}

Youth Poll 1 (Apr 01)

Youth Poll 2 (Aug 01)

Youth Poll 3 (Nov 01)

Youth Poll 4 (Nov 02)

Youth Poll 5 (June 03)

Youth Poll 6 (Nov 03)

Youth Poll 7 (May 04)

Youth Poll 8 (Nov 04)

\begin{tabular}{ccccccccc}
\hline \multicolumn{10}{c}{ Geographic Region } \\
$\begin{array}{c}\text { New } \\
\text { England }\end{array}$ & $\begin{array}{c}\text { Mid } \\
\text { Atlantic }\end{array}$ & $\begin{array}{c}\text { East } \\
\text { North } \\
\text { Central }\end{array}$ & $\begin{array}{c}\text { West } \\
\text { North } \\
\text { Central }\end{array}$ & $\begin{array}{c}\text { South } \\
\text { Atlantic }\end{array}$ & $\begin{array}{c}\text { East } \\
\text { South } \\
\text { Central }\end{array}$ & $\begin{array}{c}\text { West } \\
\text { South } \\
\text { Central }\end{array}$ & Mountain & Pacific \\
QNA & QNA & QNA & QNA & QNA & QNA & QNA & QNA & QNA \\
QNA & QNA & QNA & QNA & QNA & QNA & QNA & QNA & QNA \\
$\ddagger$ & $\ddagger$ & $\ddagger$ & $\ddagger$ & $\ddagger$ & $\ddagger$ & $\ddagger$ & $\ddagger$ & $\ddagger$ \\
$\ddagger$ & $\ddagger$ & $\ddagger$ & $\ddagger$ & $\ddagger$ & $\ddagger$ & $\ddagger$ & $\ddagger$ & $\ddagger$ \\
$\ddagger$ & 5.5 & 4.7 & 5.3 & 5.4 & $\ddagger$ & 5.5 & 5.4 & 5.1 \\
$\ddagger$ & 5.5 & 4.5 & 4.8 & 5.4 & 5.2 & 5.3 & 4.7 & 5.0 \\
$\ddagger$ & 4.8 & 4.5 & $\ddagger$ & 5.4 & $\ddagger$ & 4.6 & $\ddagger$ & 4.7 \\
$\ddagger$ & 5.0 & 4.7 & 5.1 & 5.2 & $\ddagger$ & 5.0 & 4.8 & 5.0 \\
\hline
\end{tabular}

${ }^{99}$ ‡Reporting standard not met (too few cases); QNA: Question Not Asked

Note: Changes in sample size and stratification were implemented following November 2002 Youth Poll.

Source: Department of Defense Polls, JAMRS, 2001-2004 (Question: KW2).

Page A-99 DoD May 2004 Youth Poll 


\section{U.S. Military Knowledge}

JAMRS

TABLE 20-5. Youth U.S. Military knowledge, by high school grades: $2001-2004^{100}$

\begin{tabular}{|c|c|c|c|c|c|c|c|}
\hline \multicolumn{8}{|c|}{ Male and Female (age 16-21) } \\
\hline \multirow{2}{*}{$\begin{array}{l}\text { (mean) } \\
\text { Year }\end{array}$} & \multicolumn{7}{|c|}{ High School Grades } \\
\hline & $\begin{array}{l}\text { Mostly } \\
\text { A's }\end{array}$ & $\begin{array}{l}\text { Mostly A's } \\
\text { \& B's }\end{array}$ & $\begin{array}{l}\text { Mostly } \\
\text { B's }\end{array}$ & $\begin{array}{l}\text { Mostly B's } \\
\text { \& C's }\end{array}$ & $\begin{array}{l}\text { Mostly } \\
\text { C's }\end{array}$ & $\begin{array}{l}\text { Mostly C's } \\
\text { \& D's }\end{array}$ & $\begin{array}{l}\text { Mostly D's } \\
\text { and Lower }\end{array}$ \\
\hline Youth Poll 1 (Apr 01) & QNA & QNA & QNA & QNA & QNA & QNA & QNA \\
\hline Youth Poll 2 (Aug 01) & QNA & QNA & QNA & QNA & QNA & QNA & QNA \\
\hline Youth Poll 3 (Nov 01) & 5.8 & 5.8 & 5.8 & 5.9 & $\ddagger$ & $\ddagger$ & $\ddagger$ \\
\hline Youth Poll 4 (Nov 02) & 5.1 & 5.3 & 5.2 & 5.2 & 5.3 & $\ddagger$ & $\ddagger$ \\
\hline Youth Poll 5 (June 03) & 5.5 & 5.7 & 5.5 & 5.6 & 5.6 & 5.2 & $\ddagger$ \\
\hline Youth Poll 6 (Nov 03) & 5.4 & 5.5 & 5.3 & 5.3 & 5.2 & 6.0 & $\ddagger$ \\
\hline Youth Poll 7 (May 04) & 5.2 & 5.3 & 5.2 & 5.1 & 5.2 & 5.1 & $\ddagger$ \\
\hline Youth Poll 8 (Nov 04) & 5.3 & 5.3 & 5.2 & 5.3 & 5.4 & 5.2 & $\ddagger$ \\
\hline \multicolumn{8}{|l|}{ Male (age $16-21$ ) } \\
\hline (mean) & \multicolumn{7}{|c|}{ High School Grades } \\
\hline Year & $\begin{array}{c}\text { Mostly } \\
\text { A's }\end{array}$ & $\begin{array}{l}\text { Mostly A's } \\
\text { \& B's }\end{array}$ & $\begin{array}{l}\text { Mostly } \\
\text { B's }\end{array}$ & $\begin{array}{l}\text { Mostly B's } \\
\text { \& C's }\end{array}$ & $\begin{array}{l}\text { Mostly } \\
\text { C's }\end{array}$ & $\begin{array}{l}\text { Mostly C's } \\
\text { \& D's }\end{array}$ & $\begin{array}{l}\text { Mostly D's } \\
\text { and Lower }\end{array}$ \\
\hline Youth Poll 1 (Apr 01) & QNA & QNA & QNA & QNA & QNA & QNA & QNA \\
\hline Youth Poll 2 (Aug 01) & QNA & QNA & QNA & QNA & QNA & QNA & QNA \\
\hline Youth Poll 3 (Nov 01) & 5.8 & 6.2 & 6.2 & 6.1 & $\ddagger$ & $\ddagger$ & $\ddagger$ \\
\hline Youth Poll 4 (Nov 02) & $\ddagger$ & 5.6 & 5.6 & 5.4 & $\ddagger$ & $\ddagger$ & $\ddagger$ \\
\hline Youth Poll 5 (June 03) & 5.9 & 6.0 & 5.8 & 5.9 & 5.9 & 5.3 & $\ddagger$ \\
\hline Youth Poll 6 (Nov 03) & 5.8 & 5.9 & 5.6 & 5.6 & 5.6 & 6.2 & $\ddagger$ \\
\hline Youth Poll 7 (May 04) & 5.7 & 5.9 & 5.6 & 5.3 & $\ddagger$ & 5.4 & $\ddagger$ \\
\hline Youth Poll 8 (Nov 04) & 5.9 & 5.7 & 5.6 & 5.7 & 5.3 & $\ddagger$ & $\ddagger$ \\
\hline
\end{tabular}

\section{Females (age 16-21)}

(mean)

High School Grades

\begin{tabular}{lccccccc}
\cline { 2 - 7 } Year & $\begin{array}{c}\text { Mostly } \\
\text { A's }\end{array}$ & $\begin{array}{c}\text { Mostly A's } \\
\text { \& B's }\end{array}$ & $\begin{array}{c}\text { Mostly } \\
\text { B's }\end{array}$ & $\begin{array}{c}\text { Mostly B's } \\
\text { \& C's }\end{array}$ & $\begin{array}{c}\text { Mostly } \\
\text { C's }\end{array}$ & $\begin{array}{c}\text { Mostly C's } \\
\text { \& D's }\end{array}$ & $\begin{array}{c}\text { Mostly D's } \\
\text { and Lower }\end{array}$ \\
\hline Youth Poll 1 (Apr 01) & QNA & QNA & QNA & QNA & QNA & QNA & QNA \\
Youth Poll 2 (Aug 01) & QNA & QNA & QNA & QNA & QNA & QNA & QNA \\
Youth Poll 3 (Nov 01) & 5.7 & 5.4 & 5.4 & 5.6 & $\ddagger$ & $\ddagger$ & $\ddagger$ \\
Youth Poll 4 (Nov 02) & 4.9 & 5.0 & 4.9 & 4.8 & $\ddagger$ & $\ddagger$ & $\ddagger$ \\
Youth Poll 5 (June 03) & 5.3 & 5.4 & 5.2 & 5.1 & $\ddagger$ & $\ddagger$ & $\ddagger$ \\
Youth Poll 6 (Nov 03) & 5.1 & 5.2 & 5.0 & 4.9 & $\ddagger$ & $\ddagger$ & $\ddagger$ \\
Youth Poll 7 (May 04) & 4.8 & 4.9 & 4.8 & 4.7 & $\ddagger$ & $\ddagger$ & $\ddagger$ \\
Youth Poll 8 (Nov 04) & 5.0 & 5.0 & 4.9 & 4.9 & $\ddagger$ & $\ddagger$ & $\ddagger$ \\
\hline
\end{tabular}

100 ‡Reporting standard not met (too few cases); QNA: Question Not Asked Note: Changes in sample size and stratification were implemented following November 2002 Youth Poll. Source: Department of Defense Polls, JAMRS, 2001-2004 (Question: KW2). 


\section{Employment Difficulty}

TABLE 21-1. Youth employment difficulty: $2001-2004^{101}$

\begin{tabular}{lcccc} 
Male and Female (age 16-21) & \multicolumn{4}{c}{ Percent (\%) } \\
\cline { 2 - 4 } Year & Impossible & Very Difficult & Somewhat Difficult & Not Difficult \\
\hline Youth Poll 1 (Apr 01) & 7.1 & 12.6 & 43.2 & 35.3 \\
Youth Poll 2 (Aug 01) & 5.7 & 16.8 & 44.9 & 31.4 \\
Youth Poll 3 (Nov 01) & 6.4 & 15.2 & 45.9 & 31.2 \\
Youth Poll 4 (Nov 02) & 10.6 & 17.4 & 48.4 & 22.8 \\
Youth Poll 5 (June 03) & 9.8 & 19.9 & 49.8 & 19.2 \\
Youth Poll 6 (Nov 03) & 9.1 & 21.6 & 48.9 & 18.8 \\
Youth Poll 7 (May 04) & 10.6 & 20.8 & 49.1 & 18.0 \\
Youth Poll 8 (Nov 04) & 10.7 & 21.7 & 47.3 & 18.6 \\
\hline
\end{tabular}

Male (age 16-21)

Percent (\%)

\begin{tabular}{lcccc}
\cline { 2 - 4 } Year & Impossible & Very Difficult & Somewhat Difficult & Not Difficult \\
\hline Youth Poll 1 (Apr 01) & 7.3 & 12.0 & 39.3 & 39.4 \\
Youth Poll 2 (Aug 01) & 4.7 & 16.8 & 44.7 & 32.6 \\
Youth Poll 3 (Nov 01) & 6.9 & 15.0 & 43.6 & 33.5 \\
Youth Poll 4 (Nov 02) & 8.8 & 18.0 & 46.8 & 25.6 \\
Youth Poll 5 (June 03) & 8.6 & 20.7 & 48.5 & 20.7 \\
Youth Poll 6 (Nov 03) & 10.2 & 22.3 & 47.9 & 17.8 \\
Youth Poll 7 (May 04) & 11.4 & 20.8 & 47.3 & 19.1 \\
Youth Poll 8 (Nov 04) & 9.4 & 21.3 & 46.2 & 21.0 \\
\hline
\end{tabular}

Female (age 16-21)

Percent (\%)

\begin{tabular}{lcccc}
\cline { 2 - 4 } Year & Impossible & Very Difficult & Somewhat Difficult & Not Difficult \\
\hline Youth Poll 1 (Apr 01) & 6.9 & 13.1 & 46.7 & 31.6 \\
Youth Poll 2 (Aug 01) & 6.6 & 16.8 & 45.1 & 30.3 \\
Youth Poll 3 (Nov 01) & 5.9 & 15.4 & 48.0 & 29.1 \\
Youth Poll 4 (Nov 02) & 12.3 & 16.8 & 49.9 & 20.0 \\
Youth Poll 5 (June 03) & 11.0 & 19.1 & 51.2 & 17.6 \\
Youth Poll 6 (Nov 03) & 8.0 & 20.9 & 50.0 & 19.8 \\
Youth Poll 7 (May 04) & 9.8 & 20.9 & 50.9 & 16.9 \\
Youth Poll 8 (Nov 04) & 12.0 & 22.0 & 48.5 & 16.3
\end{tabular}

${ }^{101} \ddagger$ Reporting standard not met (too few cases); QNA: Question Not Asked

Note: Changes in sample size and stratification were implemented following November 2002 Youth Poll.

Source: Department of Defense Polls, JAMRS, 2001-2004 (Question: EMP5). 


\section{Employment Difficulty}

TABLE 21-2. Youth employment difficulty, by race/ethnicity: $2001-2004^{102}$

\begin{tabular}{lcccc} 
Male and Female (age 16-21) & \multicolumn{4}{c}{ Percent (\%) } \\
\cline { 2 - 4 } (impossible \& very difficult) & \multicolumn{4}{c}{ Race/ethnicity } \\
\cline { 2 - 4 } Year & Total & White & Black & Hispanic \\
\hline Youth Poll 1 (Apr 01) & 19.6 & 17.9 & 24.0 & 23.0 \\
Youth Poll 2 (Aug 01) & 22.5 & 18.7 & 30.9 & 31.1 \\
Youth Poll 3 (Nov 01) & 21.6 & 20.2 & 28.1 & 22.3 \\
Youth Poll 4 (Nov 02) & 28.0 & 26.7 & 36.2 & 25.1 \\
Youth Poll 5 (June 03) & 29.7 & 28.8 & 38.1 & 27.5 \\
Youth Poll 6 (Nov 03) & 30.7 & 28.6 & 41.3 & 29.9 \\
Youth Poll 7 (May 04) & 31.5 & 30.3 & 41.4 & 30.2 \\
Youth Poll 8 (Nov 04) & 32.3 & 30.2 & 42.9 & 28.8 \\
\hline
\end{tabular}

\section{Male (age 16-21)}

(impossible \& very difficult)

\section{Year}

Youth Poll 1 (Apr 01)

Youth Poll 2 (Aug 01)

Youth Poll 3 (Nov 01)

Youth Poll 4 (Nov 02)

Youth Poll 5 (June 03)

Youth Poll 6 (Nov 03)

Youth Poll 7 (May 04)

Youth Poll 8 (Nov 04)

\begin{tabular}{cccc}
\multicolumn{4}{c}{ Percent (\%) } \\
\hline \multicolumn{4}{c}{ Race/ethnicity } \\
\hline Total & White & Black & Hispanic \\
\hline 19.3 & 17.9 & 25.4 & 17.4 \\
21.4 & 17.0 & 29.6 & 33.2 \\
21.9 & 20.1 & 26.4 & 23.9 \\
26.8 & 25.6 & 34.4 & 22.9 \\
29.3 & 29.0 & 39.5 & 26.4 \\
32.5 & 29.8 & 40.8 & 34.9 \\
32.2 & 31.2 & 44.5 & 28.2 \\
30.7 & 27.4 & 41.9 & 31.4 \\
\hline
\end{tabular}

\section{Female (age 16-21)}

(impossible \& very difficult)

Year

Youth Poll 1 (Apr 01)

Youth Poll 2 (Aug 01)

Youth Poll 3 (Nov 01)

Youth Poll 4 (Nov 02)

Youth Poll 5 (June 03)

Youth Poll 6 (Nov 03)

Youth Poll 7 (May 04)

Youth Poll 8 (Nov 04)

\begin{tabular}{cc}
\hline & \\
\hline Total & Whit \\
\hline 20.0 & 17.8 \\
23.4 & 20.3 \\
21.3 & 20.2 \\
29.2 & 27.8 \\
30.1 & 28.5 \\
29.0 & 27.3 \\
30.7 & 29.4 \\
34.0 & 33.3
\end{tabular}

\section{Percent (\%)}

\section{Racelethnicity}

White Black

17.8

23.1

Hispanic

31.7

27.9

28.9

29.1

20.7

27.8

37.9

26.9

37.0

28.6

41.7

25.0

38.1

32.3

43.6

\footnotetext{
${ }^{102}$ Due to relatively small sample sizes, American Indian, Alaska Native, Asian, and Pacific Islander are included in the total but are not shown separately. Black includes African American, and Hispanic includes Latino. Race categories exclude Hispanic origin unless specified otherwise.

‡Reporting standard not met (too few cases); QNA: Question Not Asked

Note: Changes in sample size and stratification were implemented following November 2002 Youth Poll.

Source: Department of Defense Polls, JAMRS, 2001-2004 (Question: EMP5).
}

Page A-102 DoD May 2004 Youth Poll 


\section{Employment Difficulty}

JAMRS

TABLE 21-3. Youth employment difficulty, by age: $2001-2004^{103}$

\begin{tabular}{lcccccc} 
Male and Female (age 16-21) & \multicolumn{7}{c}{ Percent (\%) } \\
\cline { 2 - 7 } (impossible \& very difficult) & \multicolumn{7}{c}{ Age } \\
\cline { 2 - 7 } Year & $\mathbf{1 6}$ & $\mathbf{1 7}$ & $\mathbf{1 8}$ & $\mathbf{1 9}$ & $\mathbf{2 0}$ & $\mathbf{2 1}$ \\
\hline Youth Poll 1 (Apr 01) & 22.5 & 26.2 & 20.3 & 21.7 & 13.0 & 13.4 \\
Youth Poll 2 (Aug 01) & 27.5 & 17.6 & 20.8 & 21.0 & 23.1 & 24.9 \\
Youth Poll 3 (Nov 01) & 23.0 & 23.5 & 20.1 & 25.8 & 20.2 & 16.4 \\
Youth Poll 4 (Nov 02) & 28.7 & 35.6 & 24.5 & 21.9 & 31.9 & 25.4 \\
Youth Poll 5 (June 03) & 31.9 & 32.7 & 29.1 & 26.8 & 26.5 & 30.7 \\
Youth Poll 6 (Nov 03) & 34.9 & 34.6 & 29.4 & 29.0 & 29.8 & 25.7 \\
Youth Poll 7 (May 04) & 32.3 & 32.6 & 30.9 & 29.4 & 37.1 & 26.3 \\
Youth Poll 8 (Nov 04) & 32.7 & 33.8 & 31.3 & 32.9 & 28.5 & 34.4 \\
\hline
\end{tabular}

\begin{tabular}{lcccccc} 
Male (age 16-21) & \multicolumn{7}{c}{ Percent (\%) } \\
\cline { 2 - 7 } (impossible \& very difficult) & \multicolumn{7}{c}{ Age } \\
\cline { 2 - 7 } Year & $\mathbf{1 6}$ & $\mathbf{1 7}$ & $\mathbf{1 8}$ & $\mathbf{1 9}$ & $\mathbf{2 0}$ & $\mathbf{2 1}$ \\
\hline Youth Poll 1 (Apr 01) & 20.9 & 24.7 & 19.8 & 22.0 & 14.6 & 10.3 \\
Youth Poll 2 (Aug 01) & 24.8 & 14.1 & 22.6 & 23.3 & 21.4 & 22.4 \\
Youth Poll 3 (Nov 01) & 23.1 & 23.7 & 20.9 & 28.0 & 19.1 & 16.0 \\
Youth Poll 4 (Nov 02) & 28.6 & 29.4 & 24.7 & 23.9 & 29.4 & 24.2 \\
Youth Poll 5 (June 03) & 30.7 & 33.2 & 30.5 & 21.6 & 27.1 & 31.7 \\
Youth Poll 6 (Nov 03) & 30.6 & 35.8 & 34.5 & 32.1 & 32.6 & 28.8 \\
Youth Poll 7 (May 04) & 32.5 & 27.4 & 31.3 & 30.5 & 38.8 & 32.9 \\
Youth Poll 8 (Nov 04) & 34.7 & 32.2 & 30.3 & 31.7 & 24.2 & 30.1 \\
\hline
\end{tabular}

\begin{tabular}{lcccccc} 
Female (age 16-21) & \multicolumn{7}{c}{ Percent (\%) } \\
\cline { 2 - 7 } (impossible \& very difficult) & \multicolumn{7}{c}{ Age } \\
\cline { 2 - 7 } Year & $\mathbf{1 6}$ & $\mathbf{1 7}$ & $\mathbf{1 8}$ & $\mathbf{1 9}$ & $\mathbf{2 0}$ & $\mathbf{2 1}$ \\
\hline Youth Poll 1 (Apr 01) & 24.0 & 27.9 & 20.8 & 21.4 & 11.7 & 15.5 \\
Youth Poll 2 (Aug 01) & 30.9 & 21.3 & 19.2 & 18.9 & 24.5 & 26.7 \\
Youth Poll 3 (Nov 01) & 22.9 & 23.1 & 19.2 & 24.1 & 21.0 & 16.8 \\
Youth Poll 4 (Nov 02) & 28.9 & 41.4 & 24.2 & 20.0 & 34.5 & 26.4 \\
Youth Poll 5 (June 03) & 33.2 & 32.1 & 27.7 & 32.0 & 25.8 & 29.8 \\
Youth Poll 6 (Nov 03) & 39.2 & 33.2 & 24.3 & 25.8 & 27.2 & 22.6 \\
Youth Poll 7 (May 04) & 32.2 & 37.5 & 30.5 & 28.3 & 35.3 & 19.6 \\
Youth Poll 8 (Nov 04) & 30.5 & 35.5 & 32.4 & 34.1 & 32.8 & 38.8 \\
\hline
\end{tabular}

103 fReporting standard not met (too few cases); QNA: Question Not Asked

Note: Changes in sample size and stratification were implemented following November 2002 Youth Poll.

Source: Department of Defense Polls, JAMRS, 2001-2004 (Question: EMP5).

Page A-103 DoD May 2004 Youth Poll 


\section{Employment Difficulty}

JAMRS

TABLE 21-4. Youth employment difficulty, by geographic region: $2001-2004^{104}$

\begin{tabular}{|c|c|c|c|c|c|c|c|c|c|}
\hline Male and Female (age & $6-21)$ & & & & rcent $(\%$ & & & & \\
\hline (impossible \& very difficult) & & & & Geo & raphic $\mathbf{R}$ & gion & & & \\
\hline Year & $\begin{array}{c}\text { New } \\
\text { England }\end{array}$ & $\begin{array}{c}\text { Mid } \\
\text { Atlantic }\end{array}$ & $\begin{array}{c}\text { East } \\
\text { North } \\
\text { Central }\end{array}$ & $\begin{array}{c}\text { West } \\
\text { North } \\
\text { Central }\end{array}$ & $\begin{array}{c}\text { South } \\
\text { Atlantic }\end{array}$ & $\begin{array}{c}\text { East } \\
\text { South } \\
\text { Central }\end{array}$ & $\begin{array}{c}\text { West } \\
\text { South } \\
\text { Central }\end{array}$ & Mountain & Pacific \\
\hline Youth Poll 1 (Apr 01) & $\ddagger$ & $\ddagger$ & $\neq$ & $\neq$ & $\ddagger$ & $\ddagger$ & $\ddagger$ & $\ddagger$ & $\ddagger$ \\
\hline Youth Poll 2 (Aug 01) & $\ddagger$ & $\ddagger$ & $\ddagger$ & $\ddagger$ & $\ddagger$ & $\ddagger$ & $\ddagger$ & $\ddagger$ & $\ddagger$ \\
\hline Youth Poll 3 (Nov 01) & $\ddagger$ & $\ddagger$ & $\ddagger$ & $\ddagger$ & $\ddagger$ & $\ddagger$ & $\ddagger$ & $\ddagger$ & $\ddagger$ \\
\hline Youth Poll 4 (Nov 02) & $\ddagger$ & $\ddagger$ & $\ddagger$ & $\ddagger$ & $\ddagger$ & $\ddagger$ & $\ddagger$ & $\ddagger$ & $\ddagger$ \\
\hline Youth Poll 5 (June 03) & 41.1 & 28.9 & 28.5 & 17.5 & 31.8 & 35.0 & 30.9 & 30.4 & 29.9 \\
\hline Youth Poll 6 (Nov 03) & 23.8 & 31.2 & 27.9 & 28.6 & 28.6 & 39.9 & 34.7 & 29.5 & 32.3 \\
\hline Youth Poll 7 (May 04) & 31.6 & 28.5 & 36.2 & 25.3 & 34.8 & 35.2 & 32.6 & 21.1 & 30.6 \\
\hline Youth Poll 8 (Nov 04) & 29.4 & 31.4 & 31.0 & 37.2 & 29.8 & 40.1 & 37.7 & 29.1 & 29.7 \\
\hline Male (age 16-21) & & & & & arcent $(\%$ & & & & \\
\hline (impossible \& very difficult) & & & & Geo & aphic R & gion & & & \\
\hline Year & $\begin{array}{c}\text { New } \\
\text { England }\end{array}$ & $\begin{array}{c}\text { Mid } \\
\text { Atlantic }\end{array}$ & $\begin{array}{c}\text { East } \\
\text { North } \\
\text { Central } \\
\end{array}$ & $\begin{array}{c}\text { West } \\
\text { North } \\
\text { Central } \\
\end{array}$ & $\begin{array}{c}\text { South } \\
\text { Atlantic }\end{array}$ & $\begin{array}{c}\text { East } \\
\text { South } \\
\text { Central } \\
\end{array}$ & $\begin{array}{c}\text { West } \\
\text { South } \\
\text { Central } \\
\end{array}$ & Mountain & Pacific \\
\hline Youth Poll 1 (Apr 01) & $\ddagger$ & $\ddagger$ & $\ddagger$ & $\ddagger$ & $\ddagger$ & $\ddagger$ & $\ddagger$ & $\ddagger$ & $\ddagger$ \\
\hline Youth Poll 2 (Aug 01) & $\ddagger$ & $\ddagger$ & $\ddagger$ & $\ddagger$ & $\ddagger$ & $\ddagger$ & $\ddagger$ & $\ddagger$ & $\ddagger$ \\
\hline Youth Poll 3 (Nov 01) & $\ddagger$ & $\ddagger$ & $\ddagger$ & $\ddagger$ & $\ddagger$ & $\ddagger$ & $\ddagger$ & $\ddagger$ & $\ddagger$ \\
\hline Youth Poll 4 (Nov 02) & $\ddagger$ & $\ddagger$ & $\ddagger$ & $\ddagger$ & $\ddagger$ & $\ddagger$ & $\ddagger$ & $\ddagger$ & $\ddagger$ \\
\hline Youth Poll 5 (June 03) & $\ddagger$ & 31.6 & 28.5 & 14.5 & 32.5 & $\ddagger$ & 24.2 & 30.0 & 31.4 \\
\hline Youth Poll 6 (Nov 03) & $\ddagger$ & 34.8 & 25.7 & 29.8 & 31.4 & $\ddagger$ & 39.1 & $\ddagger$ & 35.9 \\
\hline Youth Poll 7 (May 04) & $\ddagger$ & 30.8 & 31.9 & $\ddagger$ & 36.1 & $\ddagger$ & 37.0 & $\ddagger$ & 33.4 \\
\hline Youth Poll 8 (Nov 04) & $\ddagger$ & 31.2 & 26.0 & 35.1 & 27.4 & $\ddagger$ & 39.4 & 25.3 & 27.5 \\
\hline Female (age 16-21) & & & & & arcent $(\%$ & & & & \\
\hline (impossible \& very difficult) & & & & Geo & aphic R & gion & & & \\
\hline Year & $\begin{array}{c}\text { New } \\
\text { England }\end{array}$ & $\begin{array}{c}\text { Mid } \\
\text { Atlantic }\end{array}$ & $\begin{array}{c}\text { East } \\
\text { North } \\
\text { Central } \\
\end{array}$ & $\begin{array}{c}\text { West } \\
\text { North } \\
\text { Central } \\
\end{array}$ & $\begin{array}{c}\text { South } \\
\text { Atlantic }\end{array}$ & $\begin{array}{c}\text { East } \\
\text { South } \\
\text { Central }\end{array}$ & $\begin{array}{c}\text { West } \\
\text { South } \\
\text { Central } \\
\end{array}$ & Mountain & Pacific \\
\hline Youth Poll 1 (Apr 01) & $\ddagger$ & $\ddagger$ & $\ddagger$ & $\ddagger$ & $\ddagger$ & $\ddagger$ & $\ddagger$ & $\ddagger$ & $\ddagger$ \\
\hline Youth Poll 2 (Aug 01) & $\ddagger$ & $\ddagger$ & $\ddagger$ & $\ddagger$ & $\ddagger$ & $\ddagger$ & $\ddagger$ & $\ddagger$ & $\ddagger$ \\
\hline Youth Poll 3 (Nov 01) & $\ddagger$ & $\ddagger$ & $\ddagger$ & $\ddagger$ & $\ddagger$ & $\ddagger$ & $\ddagger$ & $\ddagger$ & $\ddagger$ \\
\hline Youth Poll 4 (Nov 02) & $\ddagger$ & $\ddagger$ & $\ddagger$ & $\ddagger$ & $\ddagger$ & $\ddagger$ & $\ddagger$ & $\ddagger$ & $\ddagger$ \\
\hline Youth Poll 5 (June 03) & $\ddagger$ & 25.5 & 28.4 & 20.3 & 31.1 & $\ddagger$ & 36.9 & 30.8 & 28.1 \\
\hline Youth Poll 6 (Nov 03) & $\ddagger$ & 27.7 & 30.4 & 27.4 & 25.8 & 35.4 & 30.4 & 31.6 & 28.5 \\
\hline Youth Poll 7 (May 04) & $\ddagger$ & 25.7 & 40.6 & $\ddagger$ & 33.5 & $\ddagger$ & 28.7 & $\ddagger$ & 27.8 \\
\hline Youth Poll 8 (Nov 04) & $\ddagger$ & 31.7 & 35.4 & 39.9 & 32.1 & $\ddagger$ & 36.3 & 32.9 & 32.4 \\
\hline
\end{tabular}

${ }^{104}$ ‡Reporting standard not met (too few cases); QNA: Question Not Asked

Note: Changes in sample size and stratification were implemented following November 2002 Youth Poll.

Source: Department of Defense Polls, JAMRS, 2001-2004 (Question: EMP5).

Page A-104 DoD May 2004 Youth Poll 


\section{Employment Difficulty}

JAMRS

TABLE 21-5. Youth employment difficulty, by high school grades: $2001-2004^{105}$

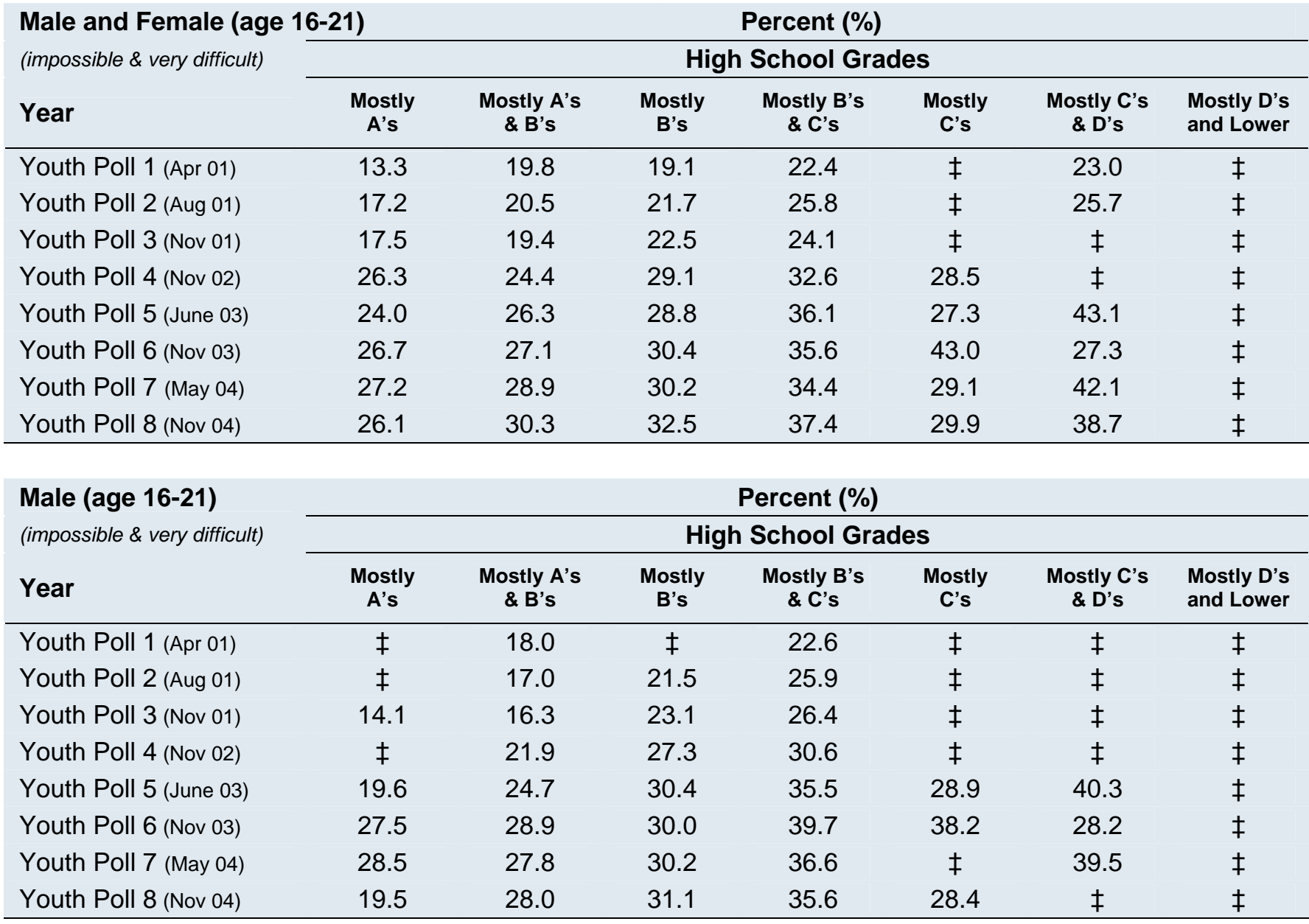

Females (age 16-21)

(impossible \& very difficult)

\begin{tabular}{lccccccc}
\cline { 2 - 7 } Year & $\begin{array}{c}\text { Mostly } \\
\text { A's }\end{array}$ & $\begin{array}{c}\text { Mostly A's } \\
\text { \& B's }\end{array}$ & $\begin{array}{c}\text { Mostly } \\
\text { B's }\end{array}$ & $\begin{array}{c}\text { Mostly B's } \\
\text { \& C's }\end{array}$ & $\begin{array}{c}\text { Mostly } \\
\text { C's }\end{array}$ & $\begin{array}{c}\text { Mostly C's } \\
\text { \& D's }\end{array}$ & $\begin{array}{c}\text { Mostly D's } \\
\text { and Lower }\end{array}$ \\
\hline Youth Poll 1 (Apr 01) & 13.6 & 21.0 & $\ddagger$ & 22.2 & $\ddagger$ & $\ddagger$ & $\ddagger$ \\
Youth Poll 2 (Aug 01) & 17.1 & 23.5 & 21.8 & 25.6 & $\ddagger$ & $\ddagger$ & $\ddagger$ \\
Youth Poll 3 (Nov 01) & 19.7 & 21.6 & 21.8 & 21.4 & $\ddagger$ & $\ddagger$ & $\ddagger$ \\
Youth Poll 4 (Nov 02) & 28.1 & 26.2 & 30.7 & 35.4 & $\ddagger$ & $\ddagger$ & $\ddagger$ \\
Youth Poll 5 (June 03) & 27.0 & 27.5 & 27.3 & 37.1 & $\ddagger$ & $\ddagger$ & $\ddagger$ \\
Youth Poll 6 (Nov 03) & 26.1 & 25.7 & 30.8 & 30.9 & $\ddagger$ & $\ddagger$ & $\ddagger$ \\
Youth Poll 7 (May 04) & 26.1 & 29.7 & 30.3 & 31.6 & $\ddagger$ & $\ddagger$ & $\ddagger$ \\
Youth Poll 8 (Nov 04) & 30.1 & 32.3 & 34.0 & 40.0 & $\ddagger$ & $\ddagger$ & $\ddagger$ \\
\hline
\end{tabular}

Percent (\%)

\section{High School Grades}

\footnotetext{
105 ‡Reporting standard not met (too few cases); QNA: Question Not Asked

Note: Changes in sample size and stratification were implemented following November 2002 Youth Poll. Source: Department of Defense Polls, JAMRS, 2001-2004 (Question: EMP5).
}

Page A-105 DoD May 2004 Youth Poll 


\section{Job Pay Comparisons}

TABLE 22-1. Youth job pay comparisons: $2001-2004^{106}$

\begin{tabular}{lccc} 
Male and Female (age 16-21) & \multicolumn{3}{c}{ Percent (\%) } \\
\cline { 2 - 4 } Year & Military & Civilian & Equally in both \\
\hline Youth Poll 1 (Apr 01) & QNA & QNA & QNA \\
Youth Poll 2 (Aug 01) & QNA & QNA & QNA \\
Youth Poll 3 (Nov 01) & QNA & QNA & QNA \\
Youth Poll 4 (Nov 02) & 21.1 & 17.5 & 60.3 \\
Youth Poll 5 (June 03) & 25.6 & 19.1 & 53.7 \\
Youth Poll 6 (Nov 03) & 20.6 & 18.2 & 59.9 \\
Youth Poll 7 (May 04) & 24.4 & 14.9 & 59.1 \\
Youth Poll 8 (Nov 04) & 19.7 & 20.2 & 59.2 \\
\hline
\end{tabular}

Male (age 16-21)

Percent (\%)

\begin{tabular}{lccc}
\cline { 2 - 3 } Year & Military & Civilian & Equally in both \\
\hline Youth Poll 1 (Apr 01) & QNA & QNA & QNA \\
Youth Poll 2 (Aug 01) & QNA & QNA & QNA \\
Youth Poll 3 (Nov 01) & QNA & QNA & QNA \\
Youth Poll 4 (Nov 02) & 18.2 & 24.0 & 56.6 \\
Youth Poll 5 (June 03) & 21.6 & 24.8 & 52.0 \\
Youth Poll 6 (Nov 03) & 18.9 & 23.9 & 56.4 \\
Youth Poll 7 (May 04) & 23.3 & 19.1 & 56.3 \\
Youth Poll 8 (Nov 04) & 16.8 & 25.5 & 56.7 \\
\hline
\end{tabular}

Female (age 16-21)

Year

Youth Poll 1 (Apr 01)

Youth Poll 2 (Aug 01)

Youth Poll 3 (Nov 01)

Youth Poll 4 (Nov 02)

Youth Poll 5 (June 03)

Youth Poll 6 (Nov 03)

Youth Poll 7 (May 04)

Youth Poll 8 (Nov 04)
Percent (\%)

\begin{tabular}{ccc} 
Military & Civilian & Equally in both \\
QNA & QNA & QNA \\
QNA & QNA & QNA \\
QNA & QNA & QNA \\
24.0 & 11.2 & 63.9 \\
29.7 & 13.2 & 55.4 \\
22.3 & 12.4 & 63.5 \\
25.5 & 10.6 & 62.1 \\
22.6 & 14.7 & 61.9 \\
\hline
\end{tabular}

\footnotetext{
${ }^{106}$ ‡Reporting standard not met (too few cases); QNA: Question Not Asked

Note: Changes in sample size and stratification were implemented following November 2002 Youth Poll.

Source: Department of Defense Polls, JAMRS, 2001-2004 (Question: IND2).
} 


\section{Job Pay Comparisons}

TABLE 22-2. Youth job pay comparisons, by racelethnicity: $2001-2004^{107}$

\begin{tabular}{lccc} 
Male and Female (age 16-21) & \multicolumn{3}{c}{ Percent (\%) } \\
\cline { 2 - 4 } (military) & \multicolumn{3}{c}{ Race/ethnicity } \\
\cline { 2 - 4 } Year & White & Black & Hispanic \\
\hline Youth Poll 1 (Apr 01) & QNA & QNA & QNA \\
Youth Poll 2 (Aug 01) & QNA & QNA & QNA \\
Youth Poll 3 (Nov 01) & 19.0 & 30.2 & QNA \\
Youth Poll 4 (Nov 02) & 23.6 & 32.5 & 28.7 \\
Youth Poll 5 (June 03) & 19.4 & 22.3 & 22.9 \\
Youth Poll 6 (Nov 03) & 22.1 & 33.6 & 26.8 \\
Youth Poll 7 (May 04) & 17.6 & 24.8 & 22.5 \\
Youth Poll 8 (Nov 04) & & & \\
\hline
\end{tabular}

\begin{tabular}{lccc} 
Male (age 16-21) & \multicolumn{3}{c}{ Percent (\%) } \\
\cline { 2 - 4 } (military) & \multicolumn{3}{c}{ Racelethnicity } \\
\cline { 2 - 4 } Year & White & Black & Hispanic \\
\hline Youth Poll 1 (Apr 01) & QNA & QNA & QNA \\
Youth Poll 2 (Aug 01) & QNA & QNA & QNA \\
Youth Poll 3 (Nov 01) & QNA & QNA & QNA \\
Youth Poll 4 (Nov 02) & 16.2 & 23.5 & 20.6 \\
Youth Poll 5 (June 03) & 17.8 & 33.7 & 26.7 \\
Youth Poll 6 (Nov 03) & 17.5 & 24.0 & 20.1 \\
Youth Poll 7 (May 04) & 20.9 & 31.4 & 27.2 \\
Youth Poll 8 (Nov 04) & 15.4 & 21.0 & 19.5 \\
\hline
\end{tabular}

\begin{tabular}{lccc} 
Female (age 16-21) & \multicolumn{3}{c}{ Percent (\%) } \\
\cline { 2 - 4 } (military) & Wace/ethnicity \\
\cline { 2 - 4 } Year & QNA & Black & Hispanic \\
\hline Youth Poll 1 (Apr 01) & QNA & QNA & QNA \\
Youth Poll 2 (Aug 01) & QNA & QNA & QNA \\
Youth Poll 3 (Nov 01) & 21.9 & 36.7 & 22.5 \\
Youth Poll 4 (Nov 02) & 29.8 & 31.5 & 30.4 \\
Youth Poll 5 (June 03) & 21.5 & 20.8 & 25.6 \\
Youth Poll 6 (Nov 03) & 23.5 & 35.6 & 26.3 \\
Youth Poll 7 (May 04) & 19.9 & 27.6 & 25.7 \\
Youth Poll 8 (Nov 04) & & & \\
\hline
\end{tabular}

\footnotetext{
${ }^{107}$ Due to relatively small sample sizes, American Indian, Alaska Native, Asian, and Pacific Islander are included in the total but are not shown separately. Black includes African American, and Hispanic includes Latino. Race categories exclude Hispanic origin unless specified otherwise.

†Reporting standard not met (too few cases); QNA: Question Not Asked

Note: Changes in sample size and stratification were implemented following November 2002 Youth Poll.

Source: Department of Defense Polls, JAMRS, 2001-2004 (Question: IND2).
} 


\section{Job Pay Comparisons}

JAMRS

TABLE 22-3. Youth job pay comparisons, by age: $2001-2004^{108}$

\begin{tabular}{lcccccc} 
Male and Female (age 16-21) & \multicolumn{7}{c}{ Percent (\%) } \\
\cline { 2 - 7 } (military) & $\mathbf{7 0}$ & $\mathbf{1 7}$ & $\mathbf{1 8}$ & $\mathbf{1 9}$ & $\mathbf{2 0}$ & $\mathbf{2 1}$ \\
\cline { 2 - 7 } Year & QNA & QNA & QNA & QNA & QNA & QNA \\
\hline Youth Poll 1 (Apr 01) & QNA & QNA & QNA & QNA & QNA & QNA \\
Youth Poll 2 (Aug 01) & QNA & QNA & QNA & QNA & QNA & QNA \\
Youth Poll 3 (Nov 01) & 16.8 & 21.7 & 24.4 & 23.3 & 22.9 & 17.5 \\
Youth Poll 4 (Nov 02) & 24.5 & 27.4 & 29.2 & 27.1 & 24.4 & 20.8 \\
Youth Poll 5 (June 03) & 24.2 & 17.2 & 23.3 & 18.6 & 17.4 & 22.9 \\
Youth Poll 6 (Nov 03) & 24.5 & 23.8 & 23.9 & 24.1 & 27.7 & 22.5 \\
Youth Poll 7 (May 04) & 20.6 & 17.3 & 20.8 & 18.7 & 15.6 & 25.1 \\
Youth Poll 8 (Nov 04) & & & & & &
\end{tabular}

\begin{tabular}{lcccccc} 
Male (age 16-21) & \multicolumn{7}{c}{ Percent (\%) } \\
\cline { 2 - 7 } (military) & $\mathbf{7 9}$ & $\mathbf{1 8}$ & $\mathbf{1 9}$ & $\mathbf{2 0}$ & $\mathbf{2 1}$ \\
\cline { 2 - 7 } Year & QNA & QNA & QNA & QNA & QNA & QNA \\
\hline Youth Poll 1 (Apr 01) & QNA & QNA & QNA & QNA & QNA & QNA \\
Youth Poll 2 (Aug 01) & QNA & QNA & QNA & QNA & QNA & QNA \\
Youth Poll 3 (Nov 01) & 11.2 & 20.3 & 21.9 & 21.8 & 20.7 & 12.6 \\
Youth Poll 4 (Nov 02) & 19.9 & 20.5 & 26.0 & 24.0 & 20.2 & 19.0 \\
Youth Poll 5 (June 03) & 24.2 & 16.4 & 17.6 & 21.1 & 19.4 & 14.5 \\
Youth Poll 6 (Nov 03) & 23.2 & 25.4 & 20.2 & 22.9 & 29.7 & 18.5 \\
Youth Poll 7 (May 04) & 18.1 & 14.7 & 19.8 & 14.8 & 12.7 & 20.8 \\
Youth Poll 8 (Nov 04) & & & & & & \\
\hline
\end{tabular}

\begin{tabular}{lcccccc} 
Female (age 16-21) & \multicolumn{7}{c}{ Percent (\%) } \\
\cline { 2 - 7 } (military) & \multicolumn{7}{c}{ Age } \\
\cline { 2 - 7 } Year & QNA & QNA & QNA & QNA & QNA & QNA \\
\hline Youth Poll 1 (Apr 01) & QNA & QNA & QNA & QNA & QNA & QNA \\
Youth Poll 2 (Aug 01) & QNA & QNA & QNA & QNA & QNA & QNA \\
Youth Poll 3 (Nov 01) & 23.0 & 23.0 & 26.9 & 24.7 & 25.2 & 21.4 \\
Youth Poll 4 (Nov 02) & 29.2 & 34.7 & 32.5 & 30.3 & 29.0 & 22.5 \\
Youth Poll 5 (June 03) & 24.3 & 17.9 & 28.9 & 16.1 & 15.6 & 31.1 \\
Youth Poll 6 (Nov 03) & 25.7 & 22.2 & 28.1 & 25.2 & 25.7 & 26.5 \\
Youth Poll 7 (May 04)6 & 23.2 & 20.0 & 21.8 & 22.6 & 18.5 & 29.4 \\
Youth Poll 8 (Nov 04) & & & & & &
\end{tabular}

\footnotetext{
${ }^{108}$ ‡Reporting standard not met (too few cases); QNA: Question Not Asked Note: Changes in sample size and stratification were implemented following November 2002 Youth Poll. Source: Department of Defense Polls, JAMRS, 2001-2004 (Question: IND2).
}

Page A-108 DoD May 2004 Youth Poll 


\section{Job Pay Comparisons}

JAMRS

TABLE 22-4. Youth job pay comparisons, by geographic region: $2001-2004^{109}$

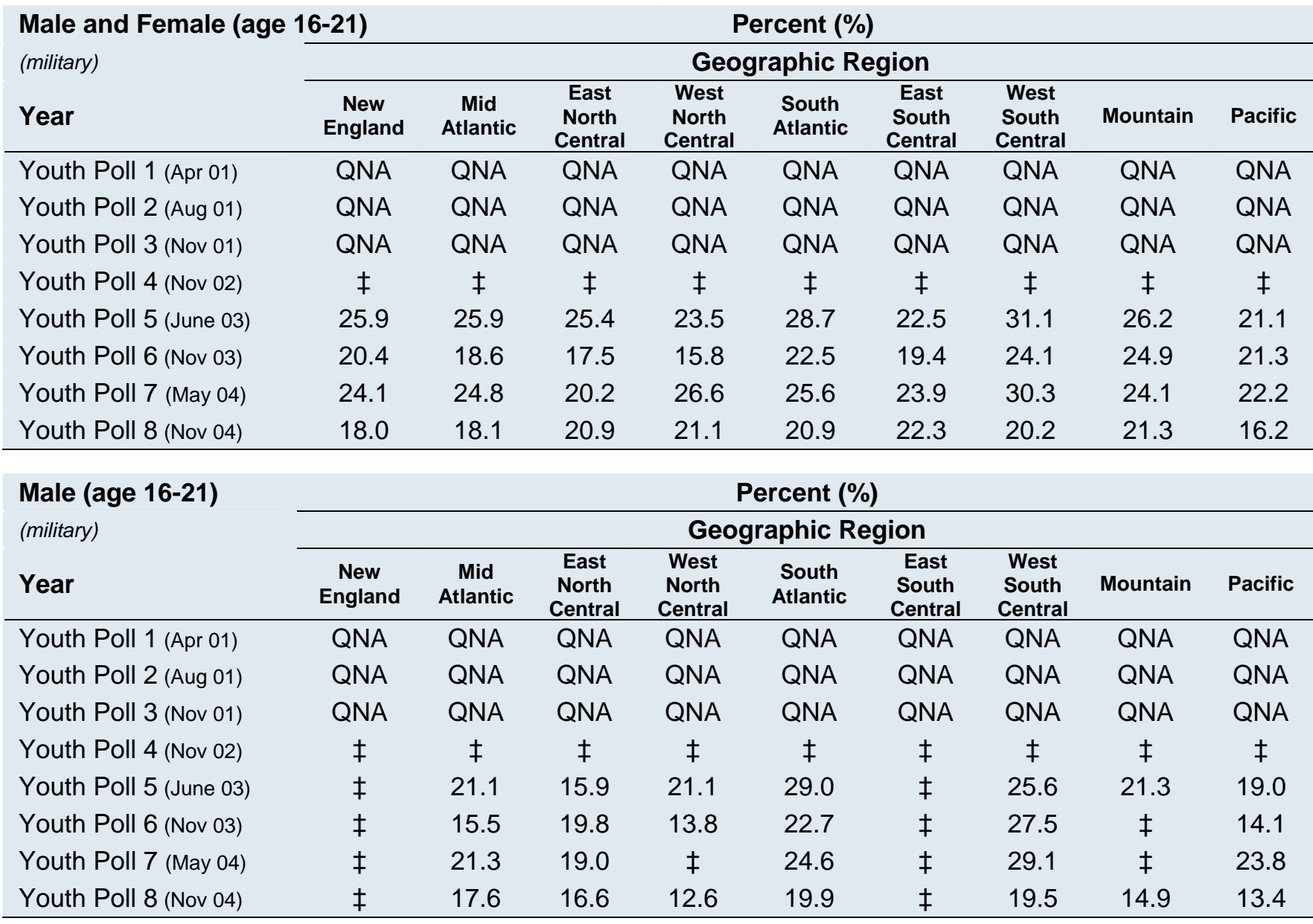

Female (age 16-21) (military)

\section{Year}

Youth Poll 1 (Apr 01)

Youth Poll 2 (Aug 01)

Youth Poll 3 (Nov 01)

Youth Poll 4 (Nov 02)

Youth Poll 5 (June 03)

Youth Poll 6 (Nov 03)

Youth Poll 7 (May 04)

Youth Poll 8 (Nov 04)

\section{Percent (\%)}

\section{Geographic Region}

\begin{tabular}{ccccccccc}
$\begin{array}{c}\text { New } \\
\text { England }\end{array}$ & $\begin{array}{c}\text { Mid } \\
\text { Atlantic }\end{array}$ & $\begin{array}{c}\text { East } \\
\text { North } \\
\text { Central }\end{array}$ & $\begin{array}{c}\text { West } \\
\text { North } \\
\text { Central }\end{array}$ & $\begin{array}{c}\text { South } \\
\text { Atlantic }\end{array}$ & $\begin{array}{c}\text { East } \\
\text { South } \\
\text { Central }\end{array}$ & $\begin{array}{c}\text { West } \\
\text { South } \\
\text { Central }\end{array}$ & Mountain & Pacific \\
QNA & QNA & QNA & QNA & QNA & QNA & QNA & QNA & QNA \\
QNA & QNA & QNA & QNA & QNA & QNA & QNA & QNA & QNA \\
QNA & QNA & QNA & QNA & QNA & QNA & QNA & QNA & QNA \\
$\ddagger$ & $\ddagger$ & $\ddagger$ & $\ddagger$ & $\ddagger$ & $\ddagger$ & $\ddagger$ & $\ddagger$ & $\ddagger$ \\
$\ddagger$ & 31.9 & 32.6 & 25.6 & 28.4 & $\ddagger$ & 35.8 & 32.3 & 23.5 \\
$\ddagger$ & 21.5 & 15.0 & 17.9 & 22.4 & 18.3 & 20.8 & 34.1 & 28.6 \\
$\ddagger$ & 29.0 & 21.5 & $\ddagger$ & 26.5 & $\ddagger$ & 31.4 & $\ddagger$ & 20.6 \\
$\ddagger$ & 18.7 & 24.8 & 32.1 & 21.8 & $\ddagger$ & 20.8 & 27.7 & 19.7 \\
\hline
\end{tabular}

\footnotetext{
${ }^{109}$ ‡Reporting standard not met (too few cases); QNA: Question Not Asked

Note: Changes in sample size and stratification were implemented following November 2002 Youth Poll.

Source: Department of Defense Polls, JAMRS, 2001-2004 (Question: IND2).
} 


\section{Job Pay Comparisons}

JAMRS

TABLE 22-5. Youth job pay comparisons, by high school grades: $2001-2004^{110}$

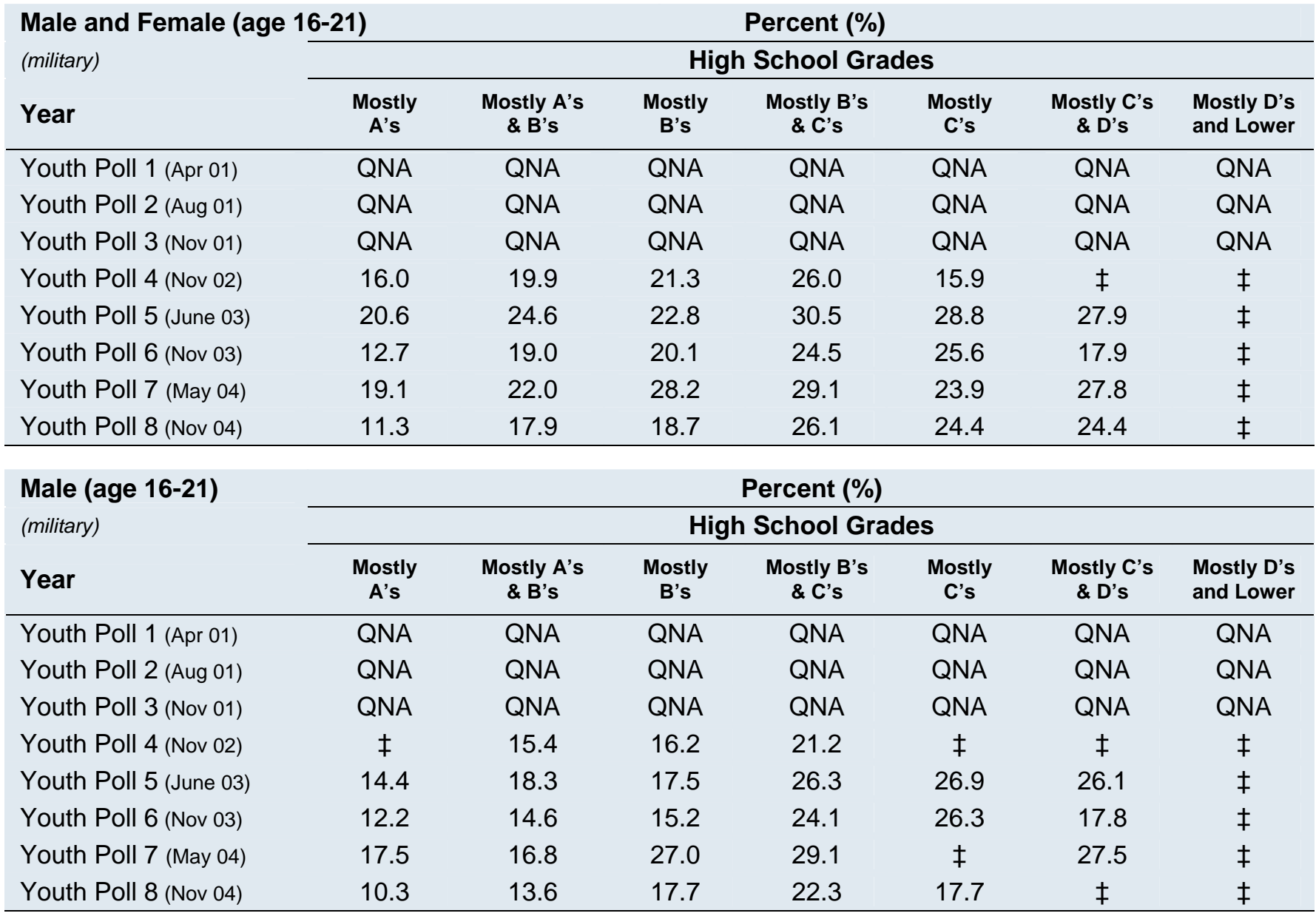

Females (age 16-21)

(military)

\section{Year}

Youth Poll 1 (Apr 01)

Youth Poll 2 (Aug 01)

Youth Poll 3 (Nov 01)

Youth Poll 4 (Nov 02)

Youth Poll 5 (June 03)

Youth Poll 6 (Nov 03)

Youth Poll 7 (May 04)

Youth Poll 8 (Nov 04)
Percent (\%)

\section{High School Grades}

\begin{tabular}{ccccccc}
$\begin{array}{c}\text { Mostly } \\
\text { A's }\end{array}$ & $\begin{array}{c}\text { Mostly A's } \\
\text { \& B's }\end{array}$ & $\begin{array}{c}\text { Mostly } \\
\text { B's }\end{array}$ & $\begin{array}{c}\text { Mostly B's } \\
\text { \& C's }\end{array}$ & $\begin{array}{c}\text { Mostly } \\
\text { C's }\end{array}$ & $\begin{array}{c}\text { Mostly C's } \\
\text { \& D's }\end{array}$ & $\begin{array}{c}\text { Mostly D's } \\
\text { and Lower }\end{array}$ \\
\hline QNA & QNA & QNA & QNA & QNA & QNA & QNA \\
QNA & QNA & QNA & QNA & QNA & QNA & QNA \\
QNA & QNA & QNA & QNA & QNA & QNA & QNA \\
18.0 & 23.1 & 25.9 & 33.0 & $\ddagger$ & $\ddagger$ & $\ddagger$ \\
24.7 & 29.2 & 27.6 & 37.1 & $\ddagger$ & $\ddagger$ & $\ddagger$ \\
13.1 & 22.2 & 25.0 & 25.0 & $\ddagger$ & $\ddagger$ & $\ddagger$ \\
20.2 & 25.5 & 29.6 & 29.2 & $\ddagger$ & $\ddagger$ & $\ddagger$ \\
11.9 & 21.6 & 19.7 & 31.4 & $\ddagger$ & $\ddagger$ & $\ddagger$
\end{tabular}

${ }^{110}$ ‡Reporting standard not met (too few cases); QNA: Question Not Asked

Note: Changes in sample size and stratification were implemented following November 2002 Youth Poll.

Source: Department of Defense Polls, JAMRS, 2001-2004 (Question: IND2). 


\section{Economic Outlook}

TABLE 23-1. Youth's economic outlook: $2001-2004^{111}$

\begin{tabular}{lccc} 
Male and Female (age 16-21) & \multicolumn{3}{c}{ Percent (\%) } \\
\cline { 2 - 4 } Year & Better Than & Worse Than & About the Same \\
\cline { 2 - 4 } & QNA & QNA & QNA \\
\hline Youth Poll 1 (Apr 01) & QNA & QNA & QNA \\
Youth Poll 2 (Aug 01) & QNA & QNA & QNA \\
Youth Poll 3 (Nov 01) & 42.6 & 24.2 & 32.8 \\
Youth Poll 4 (Nov 02) & 47.2 & 20.8 & 31.4 \\
Youth Poll 5 (June 03) & 41.8 & 25.6 & 31.8 \\
Youth Poll 6 (Nov 03) & 37.5 & 26.7 & 34.6 \\
Youth Poll 7 (May 04) & 35.0 & 29.5 & 35.0 \\
Youth Poll 8 (Nov 04) & & & \\
\hline
\end{tabular}

Male (age 16-21)

Percent (\%)

\begin{tabular}{lccc}
\cline { 2 - 4 } Year & Better Than & Worse Than & About the Same \\
\hline Youth Poll 1 (Apr 01) & QNA & QNA & QNA \\
Youth Poll 2 (Aug 01) & QNA & QNA & QNA \\
Youth Poll 3 (Nov 01) & QNA & QNA & QNA \\
Youth Poll 4 (Nov 02) & 50.9 & 19.2 & 29.3 \\
Youth Poll 5 (June 03) & 51.5 & 17.7 & 29.7 \\
Youth Poll 6 (Nov 03) & 46.2 & 21.8 & 30.8 \\
Youth Poll 7 (May 04) & 42.6 & 22.8 & 33.0 \\
Youth Poll 8 (Nov 04) & 39.3 & 27.4 & 32.8 \\
\hline
\end{tabular}

Female (age 16-21)

Percent (\%)

Year

Better Than

Worse Than

About the Same

Youth Poll 1 (Apr 01)

Youth Poll 2 (Aug 01)

QNA

QNA

Youth Poll 3 (Nov 01)

Youth Poll 4 (Nov 02)

QNA

QNA

QNA

Youth Poll 5 (June 03)

34.6

QNA

QNA

Youth Poll 6 (Nov 03)

42.8

QNA

QNA

Youth Poll 7 (May 04)

37.3

29.0

36.2

Youth Poll 8 (Nov 04)

32.1

24.0

33.1

30.7

29.5

32.7

30.7

36.2

31.6

37.1

\footnotetext{
${ }^{111} \ddagger$ Reporting standard not met (too few cases); QNA: Question Not Asked

Note: Changes in sample size and stratification were implemented following November 2002 Youth Poll.

Source: Department of Defense Polls, JAMRS, 2001-2004 (Question: IND3).
} 


\section{Economic Outlook}

TABLE 23-2. Youth's economic outlook, by race/ethnicity: $2001-2004^{112}$

\begin{tabular}{lccc} 
Male and Female (age 16-21) & \multicolumn{3}{c}{ Percent (\%) } \\
\cline { 2 - 4 } (better) & Race/ethnicity \\
\cline { 2 - 4 } Year & QNite & Black & Hispanic \\
\hline Youth Poll 1 (Apr 01) & QNA & QNA & QNA \\
Youth Poll 2 (Aug 01) & QNA & QNA & QNA \\
Youth Poll 3 (Nov 01) & 45.6 & 30.6 & QNA \\
Youth Poll 4 (Nov 02) & 49.6 & 37.7 & 41.3 \\
Youth Poll 5 (June 03) & 45.9 & 29.9 & 35.7 \\
Youth Poll 6 (Nov 03) & 39.9 & 28.9 & 35.2 \\
Youth Poll 7 (May 04) & 39.0 & 24.8 & 30.5 \\
Youth Poll 8 (Nov 04) & & & \\
\hline
\end{tabular}

\begin{tabular}{lccc} 
Male (age 16-21) & \multicolumn{3}{c}{ Percent (\%) } \\
\cline { 2 - 4 } (better) & Racelethnicity \\
\cline { 2 - 4 } Year & White & Black & Hispanic \\
\hline Youth Poll 1 (Apr 01) & QNA & QNA & QNA \\
Youth Poll 2 (Aug 01) & QNA & QNA & QNA \\
Youth Poll 3 (Nov 01) & 54.9 & 39.8 & QNA \\
Youth Poll 4 (Nov 02) & 54.7 & 39.5 & 43.5 \\
Youth Poll 5 (June 03) & 51.6 & 31.5 & 45.9 \\
Youth Poll 6 (Nov 03) & 44.8 & 32.6 & 35.0 \\
Youth Poll 7 (May 04) & 44.0 & 25.7 & 40.5 \\
Youth Poll 8 (Nov 04) & & & 33.0 \\
\hline
\end{tabular}

Male (age 16-21)

(better)

Year

Youth Poll 1 (Apr 01)

Youth Poll 2 (Aug 01)

Youth Poll 3 (Nov 01)

Youth Poll 4 (Nov 02)

Youth Poll 5 (June 03)

Youth Poll 6 (Nov 03)

Youth Poll 7 (May 04)

Youth Poll 8 (Nov 04)

\section{Percent (\%)}

Race/ethnicity

White Black

QNA

QNA

QNA

36.4

44.3

40.0

QNA

QNA

QNA

21.5

36.2

28.6

25.7

24.2
Hispanic

QNA

QNA

QNA

39.5

45.4

35.5

29.8

27.8

\footnotetext{
${ }^{112}$ Due to relatively small sample sizes, American Indian, Alaska Native, Asian, and Pacific Islander are included in the total but are not shown separately. Black includes African American, and Hispanic includes Latino. Race categories exclude Hispanic origin unless specified otherwise.

†Reporting standard not met (too few cases); QNA: Question Not Asked

Note: Changes in sample size and stratification were implemented following November 2002 Youth Poll.

Source: Department of Defense Polls, JAMRS, 2001-2004 (Question: IND3).
} 


\section{Economic Outlook}

JAMRS

TABLE 23-3. Youth's economic outlook, by age: $2001-2004^{113}$

\begin{tabular}{lcccccc} 
Male and Female (age 16-21) & \multicolumn{7}{c}{ Percent (\%) } \\
\cline { 2 - 7 } (better) & $\mathbf{7}$ & $\mathbf{1 7}$ & $\mathbf{1 8}$ & $\mathbf{1 9}$ & $\mathbf{2 0}$ & $\mathbf{2 1}$ \\
\cline { 2 - 7 } Year & QNA & QNA & QNA & QNA & QNA & QNA \\
\hline Youth Poll 1 (Apr 01) & QNA & QNA & QNA & QNA & QNA & QNA \\
Youth Poll 2 (Aug 01) & QNA & QNA & QNA & QNA & QNA & QNA \\
Youth Poll 3 (Nov 01) & 42.3 & 45.2 & 40.7 & 43.8 & 39.6 & 44.2 \\
Youth Poll 4 (Nov 02) & 45.2 & 48.8 & 48.4 & 47.0 & 47.1 & 46.7 \\
Youth Poll 5 (June 03) & 40.8 & 41.5 & 39.7 & 43.4 & 40.2 & 45.5 \\
Youth Poll 6 (Nov 03) & 41.0 & 36.6 & 37.7 & 35.7 & 32.0 & 41.0 \\
Youth Poll 7 (May 04) & 36.1 & 33.8 & 31.8 & 35.1 & 36.9 & 36.3 \\
Youth Poll 8 (Nov 04) & & & & & & \\
\hline
\end{tabular}

\begin{tabular}{lcccccc} 
Male (age 16-21) & \multicolumn{7}{c}{ Percent (\%) } \\
\cline { 2 - 7 } (better) & \multicolumn{7}{c}{$\mathbf{c}$} \\
\cline { 2 - 7 } Year & QNA & $\mathbf{1 7}$ & $\mathbf{1 8}$ & $\mathbf{1 9}$ & $\mathbf{2 0}$ & $\mathbf{2 1}$ \\
\hline Youth Poll 1 (Apr 01) & QNA & QNA & QNA & QNA & QNA & QNA \\
Youth Poll 2 (Aug 01) & QNA & QNA & QNA & QNA & QNA & QNA \\
Youth Poll 3 (Nov 01) & 47.1 & 52.1 & 46.6 & 55.4 & 53.1 & 51.2 \\
Youth Poll 4 (Nov 02) & 51.5 & 50.2 & 53.0 & 51.9 & 50.2 & 52.4 \\
Youth Poll 5 (June 03) & 45.2 & 46.8 & 45.0 & 48.0 & 41.1 & 51.4 \\
Youth Poll 6 (Nov 03) & 47.7 & 45.1 & 41.1 & 45.8 & 33.7 & 42.3 \\
Youth Poll 7 (May 04) & 39.0 & 36.7 & 36.2 & 36.9 & 46.1 & 41.1 \\
Youth Poll 8 (Nov 04) & & & & & & QNA \\
\hline
\end{tabular}

\begin{tabular}{lcccccc} 
Female (age 16-21) & \multicolumn{7}{c}{ Percent (\%) } \\
\cline { 2 - 7 } (better) & $\mathbf{7 9}$ & $\mathbf{1 7}$ & $\mathbf{1 8}$ & $\mathbf{1 9}$ & $\mathbf{2 0}$ & $\mathbf{2 1}$ \\
\cline { 2 - 7 } Year & QNA & QNA & QNA & QNA & QNA & QNA \\
\hline Youth Poll 1 (Apr 01) & QNA & QNA & QNA & QNA & QNA & QNA \\
Youth Poll 2 (Aug 01) & QNA & QNA & QNA & QNA & QNA & QNA \\
Youth Poll 3 (Nov 01) & 37.1 & 38.7 & 34.6 & 33.4 & 24.9 & 38.6 \\
Youth Poll 4 (Nov 02) & 38.8 & 47.3 & 43.6 & 42.2 & 43.6 & 41.3 \\
Youth Poll 5 (June 03) & 36.3 & 35.7 & 34.6 & 38.7 & 39.3 & 39.8 \\
Youth Poll 6 (Nov 03) & 34.2 & 28.3 & 33.9 & 25.5 & 30.4 & 39.8 \\
Youth Poll 7 (May 04) & 33.0 & 30.8 & 27.4 & 33.4 & 27.6 & 31.4 \\
Youth Poll 8 (Nov 04) & & & & & &
\end{tabular}

\footnotetext{
${ }^{113}$ ‡Reporting standard not met (too few cases); QNA: Question Not Asked

Note: Changes in sample size and stratification were implemented following November 2002 Youth Poll.

Source: Department of Defense Polls, JAMRS, 2001-2004 (Question: IND3).
} 


\section{Economic Outlook}

JAMRS

TABLE 23-4. Youth's economic outlook, by geographic region: $2001-2004^{114}$

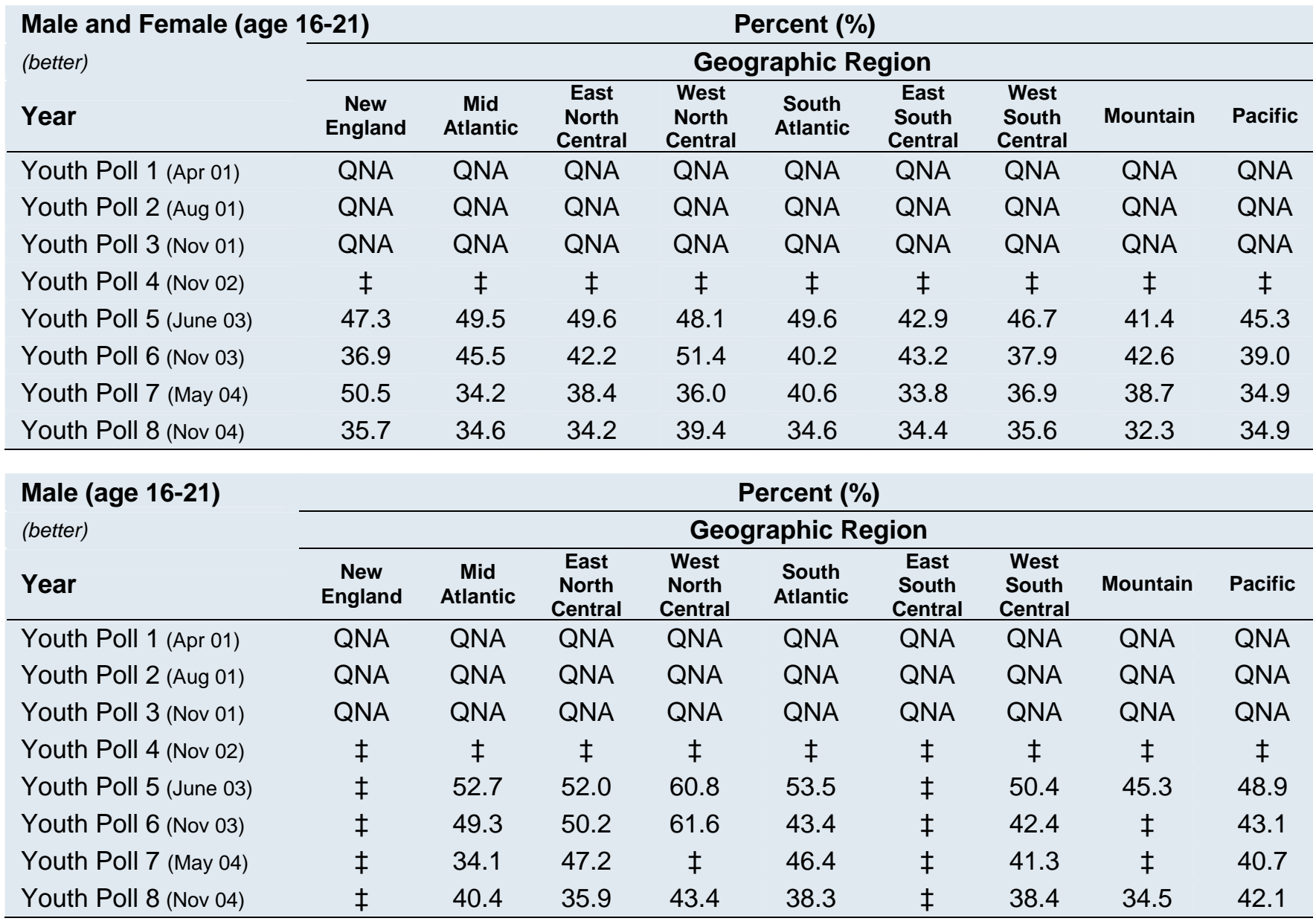

Female (age 16-21)

(better)

Year

Youth Poll 1 (Apr 01)

Youth Poll 2 (Aug 01)

Youth Poll 3 (Nov 01)

Youth Poll 4 (Nov 02)

Youth Poll 5 (June 03)

Youth Poll 6 (Nov 03)

Youth Poll 7 (May 04)

Youth Poll 8 (Nov 04)
Percent (\%)

\section{Geographic Region}

\begin{tabular}{ccccccccc}
$\begin{array}{c}\text { New } \\
\text { England }\end{array}$ & $\begin{array}{c}\text { Mid } \\
\text { Atlantic }\end{array}$ & $\begin{array}{c}\text { East } \\
\text { North } \\
\text { Central }\end{array}$ & $\begin{array}{c}\text { West } \\
\text { North } \\
\text { Central }\end{array}$ & $\begin{array}{c}\text { South } \\
\text { Atlantic }\end{array}$ & $\begin{array}{c}\text { East } \\
\text { South } \\
\text { Central }\end{array}$ & $\begin{array}{c}\text { West } \\
\text { South } \\
\text { Central }\end{array}$ & Mountain & Pacific \\
\hline QNA & QNA & QNA & QNA & QNA & QNA & QNA & QNA & QNA \\
QNA & QNA & QNA & QNA & QNA & QNA & QNA & QNA & QNA \\
QNA & QNA & QNA & QNA & QNA & QNA & QNA & QNA & QNA \\
$\ddagger$ & $\ddagger$ & $\ddagger$ & $\ddagger$ & $\ddagger$ & $\ddagger$ & $\ddagger$ & $\ddagger$ & $\ddagger$ \\
$\ddagger$ & 45.6 & 47.8 & 36.4 & 45.6 & $\ddagger$ & 43.4 & 36.7 & 41.2 \\
$\ddagger$ & 41.9 & 33.2 & 40.9 & 37.0 & 46.2 & 33.6 & 40.7 & 34.9 \\
$\ddagger$ & 34.3 & 29.5 & $\ddagger$ & 35.1 & $\ddagger$ & 33.0 & $\ddagger$ & 29.1 \\
$\ddagger$ & 28.7 & 32.7 & 34.2 & 31.2 & $\ddagger$ & 33.2 & 30.1 & 26.3 \\
\hline
\end{tabular}

${ }^{114}$ ‡Reporting standard not met (too few cases); QNA: Question Not Asked

Note: Changes in sample size and stratification were implemented following November 2002 Youth Poll.

Source: Department of Defense Polls, JAMRS, 2001-2004 (Question: IND3).

Page A-114 DoD May 2004 Youth Poll 


\section{Economic Outlook}

JAMRS

TABLE 23-5. Youth's economic outlook, by high school grades: $2001-2004^{115}$

\begin{tabular}{|c|c|c|c|c|c|c|c|}
\hline \multicolumn{4}{|c|}{ Male and Female (age 16-21) } & \multicolumn{4}{|c|}{ Percent (\%) } \\
\hline \multirow{2}{*}{$\begin{array}{l}\text { (better) } \\
\text { Year }\end{array}$} & \multicolumn{7}{|c|}{ High School Grades } \\
\hline & $\begin{array}{l}\text { Mostly } \\
\text { A's }\end{array}$ & $\begin{array}{l}\text { Mostly A's } \\
\text { \& B's }\end{array}$ & $\begin{array}{l}\text { Mostly } \\
\text { B's }\end{array}$ & $\begin{array}{c}\text { Mostly B's } \\
\text { \& C's }\end{array}$ & $\begin{array}{l}\text { Mostly } \\
\text { C's }\end{array}$ & $\begin{array}{l}\text { Mostly C's } \\
\text { \& D's }\end{array}$ & $\begin{array}{l}\text { Mostly D's } \\
\text { and Lower }\end{array}$ \\
\hline Youth Poll 1 (Apr 01) & QNA & QNA & QNA & QNA & QNA & QNA & QNA \\
\hline Youth Poll 2 (Aug 01) & QNA & QNA & QNA & QNA & QNA & QNA & QNA \\
\hline Youth Poll 3 (Nov 01) & QNA & QNA & QNA & QNA & QNA & QNA & QNA \\
\hline Youth Poll 4 (Nov 02) & 48.9 & 44.7 & 42.8 & 39.9 & 44.1 & $\ddagger$ & $\ddagger$ \\
\hline Youth Poll 5 (June 03) & 59.2 & 51.9 & 41.8 & 40.6 & 36.9 & 42.1 & $\ddagger$ \\
\hline Youth Poll 6 (Nov 03) & 52.7 & 43.8 & 43.2 & 35.3 & 37.9 & 40.3 & $\ddagger$ \\
\hline Youth Poll 7 (May 04) & 43.2 & 37.6 & 36.3 & 35.0 & 33.3 & 38.8 & $\ddagger$ \\
\hline Youth Poll 8 (Nov 04) & 46.4 & 36.4 & 31.9 & 30.7 & 25.9 & 29.3 & $\ddagger$ \\
\hline \multirow{3}{*}{$\begin{array}{l}\text { Male (age 16-21) } \\
\text { (better) }\end{array}$} & \multicolumn{7}{|c|}{ Percent (\%) } \\
\hline & \multicolumn{7}{|c|}{ High School Grades } \\
\hline & $\begin{array}{c}\text { Mostly } \\
\text { A's }\end{array}$ & $\begin{array}{l}\text { Mostly A's } \\
\text { \& B's }\end{array}$ & $\begin{array}{l}\text { Mostly } \\
\text { B's }\end{array}$ & $\begin{array}{l}\text { Mostly B's } \\
\text { \& C's }\end{array}$ & $\begin{array}{l}\text { Mostly } \\
\text { C's }\end{array}$ & $\begin{array}{l}\text { Mostly C's } \\
\text { \& D's }\end{array}$ & $\begin{array}{l}\text { Mostly D's } \\
\text { and Lower }\end{array}$ \\
\hline Youth Poll 1 (Apr 01) & QNA & QNA & QNA & QNA & QNA & QNA & QNA \\
\hline Youth Poll 2 (Aug 01) & QNA & QNA & QNA & QNA & QNA & QNA & QNA \\
\hline Youth Poll 3 (Nov 01) & QNA & QNA & QNA & QNA & QNA & QNA & QNA \\
\hline Youth Poll 4 (Nov 02) & $\ddagger$ & 58.5 & 49.5 & 44.8 & $\ddagger$ & $\ddagger$ & $\ddagger$ \\
\hline Youth Poll 5 (June 03) & 68.4 & 60.2 & 51.1 & 43.5 & 39.7 & 42.9 & $\ddagger$ \\
\hline Youth Poll 6 (Nov 03) & 59.3 & 49.8 & 50.0 & 39.8 & 39.2 & 42.1 & $\ddagger$ \\
\hline Youth Poll 7 (May 04) & 50.0 & 43.3 & 40.3 & 42.4 & $\ddagger$ & 46.3 & $\ddagger$ \\
\hline Youth Poll 8 (Nov 04) & 51.3 & 46.3 & 38.4 & 33.4 & 28.3 & $\ddagger$ & $\ddagger$ \\
\hline
\end{tabular}

Females (age 16-21)

(better)

\section{Year}

Youth Poll 1 (Apr 01)

Youth Poll 2 (Aug 01)

Youth Poll 3 (Nov 01)

Youth Poll 4 (Nov 02)

Youth Poll 5 (June 03)

Youth Poll 6 (Nov 03)

Youth Poll 7 (May 04)

Youth Poll 8 (Nov 04)

\section{High School Grades}

\section{Percent (\%)}




\section{PAGE LEFT BLANK INTENTIONALLY}


OVERVIEW REPORT

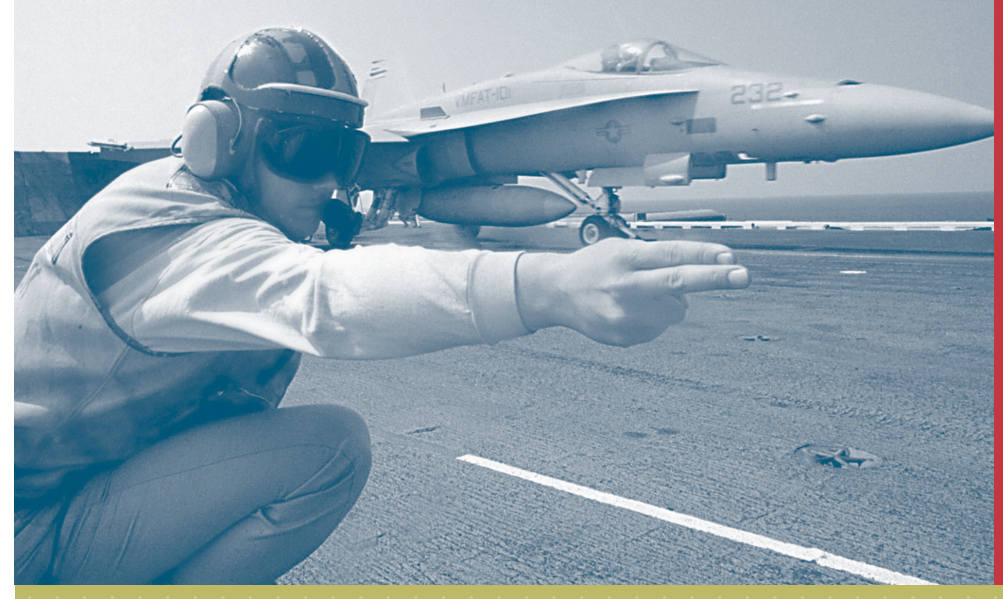

Appendix B 


\section{PAGE LEFT BLANK INTENTIONALLY}




\section{Project Overview}

This research marks the Department of Defense's (DoD) eight poll conducted among recruitaged youth. The purpose underlying the research was to expand the Department's understanding of this critical market, specifically, their attitudes about the military, and their likelihood to join.

The target population for November 2004 Youth Poll was youth between the ages of 16 and 21 who were not currently serving nor had ever served in the U.S. military. A total of 3,345 interviews were conducted through computer-assisted telephone interviews (CATI) between October 7, 2004 and November 30, 2004. The interview averaged 21 minutes in length. Final data were post-stratified by gender, age, race/ethnicity and education to reflect this population.

\section{Technical Details}

\section{Design Requirements}

The youth poll sampling frame was defined as those persons residing in the 50 states and the District of Columbia who are between the ages of 16 and 21, who had never served in the military, were not in a military delayed entry program (DEP) or one of the service academies and were not enrolled in any postsecondary Reserve Officer's Training Corps (ROTC) programs.

\section{Sample Design}

\section{Sample Stratification}

For the DoD Youth Poll, an important goal was to produce reliable estimates for racial and ethnic subgroups, specifically Whites, Blacks and Hispanics. Blacks and Hispanics are important for analytical reasons but constitute a small proportion of the total population and are dispersed throughout the country. As a result, the expected sample yield using a simple random digital dialing procedure with a sample size of 3,500 was expected to be too small to support making inferences for the subgroups at the desired level of precision. Because these subgroups are a small percentage of the population and geographically dispersed, and no single list of all the members of the subgroup is available, a simple random digit dial study was considered inadequate.

With a primary restriction in the design of the DoD Youth Poll being cost, stratified random sampling was selected as the best method. When a study involves sampling of a rare population, as shown by Waksberg (1973) ${ }^{1}$, stratification can produce a significant reduction in the level of screening and cost when (a) a high percentage of the rare population can be identified and stratified for oversampling, and when (b) these strata contain a small part of the total population (or contain a substantial portion of the rare population).

The approach that was taken involved stratifying telephone exchanges by concentration of the rare population, and over-sampling the strata with high concentrations. Under this scheme,

\footnotetext{
${ }^{1}$ Waksberg, J. (1973). The effect of stratification with differential sampling rates on attributes of subsets of the population. Pp. 429-434 in Proceedings of the Social Statistics Section. Washington, DC: American Statistical Association.
} 
auxiliary information was used to classify telephone exchanges (or banks of telephone numbers) by the proportion of members of the groups residing in these exchanges. After classifying the exchanges into strata, the telephone numbers in the exchanges with the higher proportion of rare members were sampled at a higher rate than the numbers in the other strata. If the data used to stratify the numbers is accurate, then the telephone numbers in the exchanges sampled at higher rates would be more likely to result in interviews with members of the rare subgroup. This procedure has been used in numerous past RDD surveys to improve the precision of estimates of African Americans and Hispanics.

This option however places increased attention on the sample design. Prior to data collection, the exchanges were listed according to the density concentration of the small domains to identify cut-off points. These cut-points were then used to determine the optimal stratification with the highest yield and minimal increase in design effect. The optimal cut-off point for this poll was calculated at $30 \%$.

Calculating the optimal over-sampling level was the first step. Assuming a single cost function in which the total cost of interviewing $n_{i}$ units within stratum $i, i=1,2$ is given by:

$$
\mathrm{C}=\left(\mathrm{r}_{1} \mathrm{n}_{1}+\mathrm{r}_{2} \mathrm{n}_{2}\right) \mathrm{c}_{1}+\left(\mathrm{n}_{1}+\mathrm{n}_{2}\right) \mathrm{c}_{2}
$$

where $\mathrm{n}_{\mathrm{i}}$ is the sample size in stratum $i, \mathrm{r}_{i}$ is the average amount of screening required to locate one member of the rare group in stratum $i, c_{1}$ is the average cost of a screening call, $c_{2}$ is the average cost of interviewing one member, and $\mathrm{C}$ is the total cost. If we minimize the sampling variance subject to a fixed cost, we obtain the optimum allocation sample sizes. The ratio of sample sizes is given by:

$$
\frac{n_{1}}{n_{2}}=\frac{\sigma_{1} \mathrm{~N}_{1}}{\sigma_{2} \mathrm{~N}_{2}} \sqrt{\frac{r_{2}+\frac{C_{2}}{C_{1}}}{r_{1}+\frac{C_{2}}{C_{1}}}}
$$

where $\mathrm{N}_{\mathrm{i}}$ is the population of the rare group in stratum $i$.

The optimal allocation was calculated using the above formula and used in the sample allocation for the two strata.

\section{Sample Selection}

After the allocation of the sample, two methods of systematic sample selection are available. Using a Random A methodology, the list frame is all possible 10-digit telephone numbers in blocks with one or more listed telephone numbers. From this frame, telephone numbers serving the sample area are selected with equal probability. Using a Random B methodology, telephone numbers serving the sample area are selected with probability equal to the number of listed telephone numbers in each working block. Blocks with no listed numbers have zero probability of selection in both methodologies. 
Random A samples were used for this poll because they typically provide samples with better efficiency than pure equal probability of selection (EPSEM) samples. With this approach, the counts of telephones within each working block (a block with one or more listed telephone numbers) are first examined to decide which should be included in the sample and which should be discarded. For this poll, those blocks with only one listed telephone number were also excluded so dialing would be more efficient and coverage would be marginally greater. ${ }^{2}$

The phone list vender, SSI ${ }^{\circledR}$, offers the option of protecting Random A samples against reuse. In tracking surveys, the practical consideration of not calling the same sample in subsequent time frames is a benefit that may be viewed to outweigh the potential bias of not replacing numbers. Virtually every SSI ${ }^{\circledR}$ Random A sample is marked on the database to protect against reuse for a period of nine months. The SSI ${ }^{\circledR}$ Protection System was designed to reduce the chance of selecting the same number for multiple projects or multiple waves of a single project conducted by a single research firm or by competing research firms.

\section{Interviewing Hours}

Interviews were conducted from October 7 to November 30, 2004 during the evening and weekend hours for the time zone in which the respondent lived. Specifically, interviews were conducted from 4 pm through 9 pm respondent time Sunday through Thursday, and 10 am through $6 \mathrm{pm}$ on Saturdays. The decision was made not to dial on Fridays based upon low productivity rates in past Youth Poll waves.

The low density stratum was fielded out of Wirthlin's phone center located in Orem, Utah. The high density stratum was fielded by Wirthlin's partner Directions in Research (DIR) located in San Diego, California. The two stratums were separated because DIR has specialized interviewers that are trained to conduct interviews with minorities, specifically Hispanics and African Americans.

\section{Sample Geography}

Interviews were conducted in all 50 states plus the District of Columbia.

\section{Business and Cellular Phone Numbers}

On average, an RDD sample will contain 15 to 18 percent business and cellular phone numbers. Approximately half of these numbers can be identified using SSI's Business and Cellular

\footnotetext{
${ }^{2}$ Approximately 2.5 million blocks were identified as working (having one or more listed numbers). By raising the minimum acceptable block size from 1 to 3 or more (SSI's ${ }^{\circledR}$ default), further gains in efficiency could be achieved with only minimal reduction in coverage. Blocks with 1-2 listed numbers represent only $5.9 \%$ of all working blocks and only $0.3 \%$ of all listed telephone households. These listed numbers are far more likely to be keypunch errors or White Page business listings than only the listed number in a given block. SSI ${ }^{\circledR}$ uses a default minimum block size of 3 listed numbers, but this minimum may be adjusted up or down based on the user's specifications. Users can even sample from blocks with zero listed numbers, but efficiency may fall as low as $16 \%$. Further, a $65 \%$ working phones rate with a Random B sample, a 55\% rate with Random A and as low as 30\% with an EPSEM sample should be expected.
} 
Number Purge options. SSI maintains a database of over 11 million business and cellular telephone numbers, compiled from Yellow Page directories and other special directories. Once a 10-digit telephone number was selected for a sample the status of the number generated was compared to SSI's list of known business and cellular numbers.

\section{$\underline{\text { Replicates }}$}

For this poll, the sample was identified and released in replicates (representative stand-alone mini-samples that are representative of the entire sample). When using a replicate system, the interviewers did not need to dial the entire sample as each replicate was representative of the entire sample. All replicates loaded were closed out and dialed until exhausted. A sample record was considered "exhausted" once it had obtained a final disposition, such as disconnected, complete, or refusal. So there would not be "extra" interviews, the sizes of the replicates were reduced as the interview period drew to a close.

Additionally, replicates were ordered proportionate to the sample allocation determined for the two strata. Replicates for Strata 1 and Strata 2 were released and dialed through evenly. A replicate for either strata was not allowed to be closed unless the same replicate for the other strata was exhausted as well.

\section{Quotas and Thresholds}

Because of the speed at which polls are conducted and the rate at which surveys are completed, it is often necessary to set quotas, or the minimum number of completed surveys for each area. This ensures a representative sample is obtained. Therefore, soft quotas, or targets for the minimum number of surveys to be completed, were placed on each region. The following "guides" for each region were set in place:

New England (5.06\%)

Island,

Mid-Atlantic (14.33\%)

South Atlantic (18.73\%)

South

East South Central (6.09\%)

East North Central (16.01\%)

West North Central (6.82\%)

Minnesota West South Central (10.89\%) Texas, Louisiana, Arkansas, Oklahoma

Mountain (6.33\%)

Pacific (15.75\%)
Connecticut, Maine, Massachusetts, New Hampshire, Rhode Vermont

New Jersey, New York, Pennsylvania

Delaware, Maryland, West Virginia, Virginia, North Carolina, Carolina, Georgia, Florida, District of Columbia

Mississippi, Alabama, Tennessee, Kentucky

Illinois, Indiana, Michigan, Ohio, Wisconsin

Iowa, Kansas, Missouri, Nebraska, North Dakota, South Dakota,

Arizona, Colorado, Idaho, Montana, Nevada, New Mexico, Utah, Wyoming

California, Oregon, Washington, Hawaii and Alaska

Additionally, soft quotas were placed on gender to approximate the most recent Census levels. 
Although "soft" quotas were in place for this study, no telephone numbers or interviews were discarded or terminated as a result of the quota system. They were only used as a check during the data collection phase to alert phone center staff to possible problems.

\section{$\underline{\text { Survey Implementation }}$}

\section{Screening}

Each household was screened for youth who met the following criteria:

- Youth at least 16 years old, and less than 22 years old

- Had never served in the U.S. Armed Forces

- Were not accepted for service in the U.S. Armed Forces (Service includes the active and Reserve Components of the U.S. Army, Navy, Air Force, Marine Corps, and Coast Guard)

- Were not in a Military Delayed Entry Program (DEP), college ROTC, or one of the Service academies

Polling identifies all eligible respondents in the household and resolves the selection on the initial screen call. If there was more than one person in the household who met the criteria, the respondent in the household between the ages of 16 and 21 with the most recent birthday prior to the interview date was selected. If that individual was away at college (living in a dormitory, fraternity house or temporary housing) his/her telephone number and name was requested and placed in the callback queue. There was no within household substitution of the designated respondent, even if the designated respondent did not qualify for the interview (e.g., is currently in the military, etc.).

\section{Callback Procedure}

One initial call and a maximum of nine callbacks were allowed. If a household was not reached after ten calls, another randomly selected household was substituted.

\section{Refusal Conversion}

An active program of refusal conversion was used. All initial refusals were put into a queue to be worked by a group of interviewer specialists, trained and experienced in refusal conversion. Up to an additional three callbacks, conducted at different times and days, were made. If a household was not reached after three calls or if a second refusal occurred, a "hard" refusal was recorded on the final disposition. 
Demographic Profile of Respondents

November 2004 Youth Poll Sample Yields -- HIGH DENSITY STRATUM

Business

23,956

Fax/ Cell/ Pager

14,215

Bad phone number

20,220

Final no answer

110,979

Final answering machine

9,573

Privacy manager

9,807

Non-Eligible Units

188,750

Ineligible age

48,662

Ineligible college referral number/ refused

Ineligible Military DEP, ROTC, Service Academy

Ineligible refused ethnicity

Language

9,948

Deceased/ Retired

429

Non-Eligible Respondents $\quad 59,320$

Complete

Interviews

1,665

1,665

Final busy

12,787

Designated respondent unavailable

No Contact

13,239

Indefinite callback

Definite callback

Qualified terminate

493

Interviewer terminate

245

Partial Interviews $\quad 3,789$

Final refusal

9,780

Total Refusals $\quad \mathbf{9 , 7 8 0}$

Sample Dialed

276,533

Less Non-Eligible Units

188,750

Less Non-Eligible Respondents

59,320

Eligible Phone Numbers

28,463

Completed Interviews

1,655 
November 2004 Youth Poll Sample Yields -- LOW DENSITY STRATUM

Business

Fax/ Cell/ Pager

15,604

Bad phone number

77,189

Final no answer

Final answering machine

15,389

Privacy manager

Non-Eligible Units

182,912

Ineligible age

54,381

Ineligible college referral number/ refused

Ineligible Military DEP, ROTC, Service Academy

Ineligible refused ethnicity

13

Language

Deceased/ Retired

Non-Eligible Respondents $\quad 57,149$

Complete

Interviews

1,690

Final busy

Designated respondent unavailable

No Contact

4,058

Indefinite callback

102

Definite callback

24

Qualified terminate

95

Interviewer terminate

1,021

Partial Interviews

1,242

Final refusal

16,104

Total Refusals

16,104

Sample Dialed

264,073

Less Non-Eligible Units

182,912

Less Non-Eligible Respondents

57,149

Eligible Phone Numbers

24,012

Completed Interviews

1,690 


\section{$\underline{\text { Weight Construction }}$}

There were three main phases in the creation of the weights for Youth Poll 8: (1) Base Weights, which are the inverse of the probability of a respondents' inclusion in the sample, (2) NonResponse Adjustment, in which the respondents are weighted to account for non-respondents, and (3) Poststratification, where the weights are corrected to match population totals for certain demographic characteristics.

\section{Base Weights}

The base weights are calculated as the inverse of the probability of inclusion for the telephone line. This is done using the sampled telephone lines with known eligibility (whether eligible or not). This probability of inclusion equals the number of sampled telephone lines for which the eligibility is known, divided by the total number of telephone lines. This can be calculated given that we know the total number of lines in each stratum and the distribution of sampled telephone lines per interview disposition codes.

For the "low-density" stratum, the total number of lines is approximately $182,865,000$. The (initial) sample size is calculated as the number of sampled telephone lines for which the eligibility is known. There were 177,837 sampled telephone lines with known eligibility; these can be divided in two groups: 5,969 eligible telephone lines, which include "Complete", "Designated Respondent Never Available", "Indefinite Appointment", "Definite Appointment", and "Qualified Terminate"; and 171,868 non-eligible telephone lines, which include "Business", "Fax/Cell Phone/Pager", "Bad Phone Number", "Duplicate Record”, "Ineligible Age", "Ineligible Military DEP, Service Academy, ROTC", and "Deceased/Retired".

Therefore, the probability of inclusion of a telephone line in the "low-density" stratum is, $177,837 / 182,865,000=9.73 \times 10^{-4}$ and the initial weight of a line in this stratum is the inverse of this number, 1028.

Similarly, for the "high-density" stratum there are a total of 47,505,000 telephone lines and 113,256 sampled lines with known eligibility. This includes 5,661 eligible lines and 107,595 non-eligible lines. The probability of inclusion for this stratum is $113,256 / 47,505,000=2.38 \mathrm{x}$ $10^{-3}$, with an initial weight 419 .

\begin{tabular}{l|l|l|l|l} 
Calculation of Initial weight \\
Stratum & $\begin{array}{l}\text { Sampled Lines with } \\
\text { Known Eligibility }\end{array}$ & $\begin{array}{l}\text { Lines in } \\
\text { Stratum }\end{array}$ & $\begin{array}{l}\text { Prob. of } \\
\text { Inclusion of } \\
\text { Line }\end{array}$ & $\begin{array}{l}\text { Initial } \\
\text { Weight }\end{array}$ \\
\hline Low-density Stratum & 177,837 & $\begin{array}{l}182,865,000 \\
9.73 E-04\end{array}$ & 1028 \\
High-density Stratum & 113,256 & $47,505,000$ & $2.38 \mathrm{E}-03$ & 419 \\
\hline
\end{tabular}

At this step, all the sampled lines with known eligibility within a stratum have the same, nonzero, weight even if the line is non-eligible. This weight is at the telephone line level. In order to obtain a person-level weight, and get a zero weight for the non-eligible units, this "pre-weight" is 
multiplied by the number of eligible persons for the telephone line and the number of home phone lines for the household. This number of eligible persons is zero for the non-eligible telephone lines, and now only eligible units have non-zero weights. Incomplete eligible units are adjusted by the average number of eligible persons and average number of phone lines for each respective stratum.

Household Eligible Count: Base Weight Adjustment for Eligible Persons

\begin{tabular}{l|l}
\hline Number of Eligible Persons in Tel. Line & Base Weight \\
\hline No Eligible Persons in House/Business & Initial Weight $* 0=0$ \\
One Eligible person in household & Initial Weight $* 1$ (No adjustment) \\
Two or more Eligible Persons & Initial Weight $* 2$ \\
\hline
\end{tabular}

These weights are called the "base weights" since they are, basically, the inverse of the probability of inclusion of the sampled elements, including non-respondents.

\section{Non-Response Adjustment}

The base weights are non-zero for all the eligible sampled elements, including non-respondents. This has to be rectified because there are no data for these elements and they must have a weight equal to zero. Since the "pattern" of non-response can differ for the two strata, that is to say, the likelihood of an element being a non-respondent can differ for the two strata; this adjustment must be made within each stratum.

This is accomplished by increasing the base weights of the respondents in each stratum to account for the non-respondents in their corresponding stratum. After this adjustment, the weights for the respondents are higher than the base weights and the weights for the nonrespondents are zero, leaving the sample with respondents only.

This non-response adjustment is, for each respondent in each stratum, equal to the sum of the base weights in that stratum (for all respondents and non-respondents) divided by the sum of the base weights for the respondents. Therefore, the non-response adjusted weight for a given respondent is (original base weight) $\mathrm{x}$ (sum of base weights in the corresponding stratum) / (sum of base weights for respondents in the stratum).

Nonresponse Adjustment

\begin{tabular}{l|l|l|l}
\hline Stratum & $\begin{array}{l}\text { Sum of Weights } \\
\text { for Respondents }\end{array}$ & $\begin{array}{l}\text { Sum of Weights for } \\
\text { Eligible Nonrespondents }\end{array}$ & $\begin{array}{l}\text { Nonresponse } \\
\text { Adjustment }\end{array}$ \\
\hline Low-density Stratum & $2,124,426$ & $6,240,957$ & Base Weight *3.94 \\
High-density Stratum & 845,877 & $2,007,654$ & Base Weight*3.37 \\
\hline
\end{tabular}




\section{Poststratification of Weights}

The final step in the calculation of the weights involves their modification in a way that the sample distributions of some important demographic characteristics are adjusted so that they are equal to the known distributions of the corresponding characteristics in the population. This is referred to as poststratification, and is used to reduce the variance of the estimates and to correct for under coverage in the survey of some types of units.

Poststratification adjustments were calculated by a two-dimensional raking procedure. Raking allows for the poststratification to marginal population totals of several variables simultaneously. This is one way used to ensure consistency between complete (population) count and sample data. Raking is used in situations where the interior cells of the cross tabulation are either unknown or sample sizes in some cells are too small for efficient estimation in poststratification to the whole cross-tabulation.

Four demographic characteristics, in two "raking dimensions", were used to post-stratify: Gender and Age (Raking Dimension 1), and Race/Ethnicity and Education (Raking Dimension 2). The population totals for these two cross-classifications for November of 2004 were obtained from the Current Population Survey (CPS).

\begin{tabular}{l|l|l} 
November 2004 CPS for Raking Dimension 1 \\
\hline GENDER & AGE & CPS Total \\
\hline Male & $\mathbf{1 6}$ & $2,273,208$ \\
Male & $\mathbf{1 7}$ & $2,183,652$ \\
Male & $\mathbf{1 8}$ & $1,901,588$ \\
Male & $\mathbf{1 9}$ & $1,882,455$ \\
Male & $\mathbf{2 0}$ & $1,951,693$ \\
Male & $\mathbf{2 1}$ & $1,990,817$ \\
Female & $\mathbf{1 6}$ & $2,143,767$ \\
Female & $\mathbf{1 7}$ & $2,120,402$ \\
Female & $\mathbf{1 8}$ & $1,862,944$ \\
Female & $\mathbf{1 9}$ & $1,889,076$ \\
Female & $\mathbf{2 0}$ & $1,933,791$ \\
Female & $\mathbf{2 1}$ & $1,971,901$ \\
\hline \multicolumn{3}{c}{$24,105,294$}
\end{tabular}


November 2004 CPS for Raking Dimension 2 (RACE/ETH by EDUCATION)

\begin{tabular}{llr}
\hline RACE/ETHNICITY & EDUCATION & CPS Total \\
\hline White, Non-Hispanic & Less than high school & $6,945,001$ \\
White, Non-Hispanic & High school, no college & $3,332,400$ \\
White, Non-Hispanic & Some college, but no bachelors degree & $4,684,831$ \\
White, Non-Hispanic & Bachelors degree or more & 76,055 \\
Black, Non-Hispanic & Less than high school & $1,831,316$ \\
Black, Non-Hispanic & High school, no college & 862,438 \\
Black, Non-Hispanic & Some college, but no bachelors degree & 756,149 \\
Black, Non-Hispanic & Bachelors degree or more & 18,184 \\
Hispanic & Less than high school & $2,359,608$ \\
Hispanic & High school, no college & 929,261 \\
Hispanic & Some college, but no bachelors degree & 731,149 \\
Hispanic & Bachelors degree or more & 6,378 \\
Other, Non-Hispanic & Less than high school & 774,967 \\
Other, Non-Hispanic & High school, no college & 312,920 \\
Other, Non-Hispanic & Some college, but no bachelors degree & 468,440 \\
Other, Non-Hispanic & Bachelors degree or more & 16,187 \\
\hline & & $24,105,294$
\end{tabular}

\section{$\underline{\text { Variance Estimation }}$}

The most straightforward types of samples, from a statistical standpoint at least, are simple random samples. In such samples the confidence limits for a proportion are influenced by the sample size of the sample, or particular subsample under consideration, and also by the value of the proportion.

The standard error ${ }^{3}$ of a proportion $p$ from a simple random sample of $n$ cases is equal to:

$$
\sqrt{p(1.0-p) / n}
$$

With a large number of cases, a symmetrical confidence interval around $p$ would be approximated by:

$$
p \pm z \sqrt{p(1.0-p) / n}
$$

where $z$ is the appropriate value from the $\mathrm{z}$-distribution. For a $95 \%$ confidence interval, for example, $z=1.96$.

\footnotetext{
${ }^{3}$ The standard error of an estimate is a measure of sampling error; it is defined as the standard deviation of the sampling distribution of the statistic. It is used to construct the confidence interval around the estimate.
} 


\section{Significance of Difference between Two Proportions}

In addition to estimating the sampling error around a single proportion, we often wish to test the significance of a difference between two proportions, such as the difference between the proportions of males interested in joining the military versus females. The following formula produces a statistic that can be referred to a standard normal distribution, assuming a reasonably large number of cases:

$$
z=\frac{p_{1}-p_{2}}{\sqrt{p_{e}\left(1-p_{e}\right) \frac{n_{1}+n_{2}}{n_{1} n_{2}}}}
$$

where:

$$
p_{e}=\frac{n_{1} p_{1}+n_{2} p_{2}}{n_{1}+n_{2}}
$$

and $p_{e}$ is the estimated population proportion, $p_{1}$ is the observed proportion (of male in our example) in the first group, $p_{2}$ is the observed proportion in the second group (of females in our example), $n_{1}$ is the number of cases in the first group, and $n_{2}$ is the number of cases in the second group.

\section{Variance Estimation with more Complex Designs}

The above variance estimation formulas however, are only appropriate for simple random samples. In complex samples, such as those used in the Youth Polls, that involve stratification and weighting, it is also necessary to take into account the effect that the sampling design has on the size of the standard errors.

Methods exist for correcting for this underestimation of the standard errors. Kish $(1965)^{4}$ defines a correction term called the design effect (DEFF) where:

$$
D E F F=\frac{\text { actual sampling variance }}{\text { Variance expected from a random sample }}
$$

Thus, if the actual sampling variance in a complex sample is four times as large as the sample variance from a simple random sample with the same number of cases, the DEFF is 4.0. Because confidence intervals are proportionate to the square root of the variance, the confidence interval for such a sample would be twice as large (because the square root of 4 is 2 ) as the confidence interval for a simple random sample with the same number of cases. If an estimate of design effect is available, one of the simplest correction procedures to follow is to divide the actual number of cases by the design effect (thereby depreciating the actual number to its equivalent

\footnotetext{
${ }^{4}$ Kish, L. (1965). Survey Sampling. New York: John Wiley \& Sons.
} 
value in simple random sample terms) and then employ the standard statistical procedures that are available for application to simple random samples.

\section{Significance testing for differences between fieldings of the Youth Poll}

A trend over two fieldings of the Youth Poll is basically a comparison between estimates from two independent samples. Therefore, the design effects for a single estimated proportion are appropriate. For the majority of situations in the Youth Poll, tests for changes over time were done by estimating design effects as calculated from STATA ${ }^{\circledR}$ or another of the similar data analysis software programs and dividing $n$ by the design effect to obtain an effective $n$. This effective $n$ was then used in place of the actual $n$ and the formulas appropriate for simple random sampling were conducted. Design effects for proportions and means, although not provided in this technical report, can be calculated by individual users or can be provided upon request from interested users of the data.

\section{Variance estimation procedures for November 2004 Youth Poll estimates}

To find confidence intervals and test hypotheses using the November 2004 Youth Poll data, it is necessary to find estimates of the variance for the estimated statistics, whether the statistics are means, proportions, correlations, or regression weights. Alternative approaches to finding effective $n$ sizes based on design effects, as outlined above, may be required in certain situations for certain types of statistical testing. There are a number of different approaches to estimate the variability of (complex) parameters in complex surveys; two of the more common approaches are referred to as Linearization by Taylor series expansion and Replication, both of which take into account design effects but rely on readily available computer software to remove tedious hand calculations and adjustments.

Users are cautioned not to ignore the design feature (i.e., stratification and weighting) of the data collection for this survey in their significance test. Stratification, as done in the data collection for this survey, effectively allows the calculation of variance for a statistics that is based solely on within stratum variance. This variance estimate is almost universally smaller than the one that would be obtained if the data were treated as being collected using only simple random sampling. Ignoring the stratification will typically result in an over-estimation of the variance whereby the hypothesis testing conducted is biased.

In the majority of estimations done for the November 2004 Youth Poll, the technique used by the Joint Advertising, Market Research Studies (JAMRS) to find variance estimates for the statistics reported is the Taylor-series linearization method, as implemented using the software program STATA $^{\circledR}$.

For those familiar with data analysis programs such as WESVAR ${ }^{\mathrm{TM}}, \mathrm{STATA}^{\circledR}$, SUDAAN $^{\circledR}$, or $\mathrm{SAS}^{\circledR}$, appropriate variance estimation formulas can be obtained using some relatively straightforward programming. However, the above software programs do not handle variance estimation in identical ways so users should be aware of and comfortable with the assumptions of their chosen software program. 
For users who require hypothesis testing but prefer not to use the formulas provided for hand calculation or are not familiar with one of the above listed software programs, a third option exists. JAMRS is available to handle any hypothesis testing requests that users of this data have. Service requests will be given top priority, however, all users may feel free to submit requests. All that is required is an email to either Stacy Emanuel (emanuesn@ osd.pentagon.mil) or Sean Marsh (marshsm@osd.pentagon.mil) that contains the analysis you would like to have completed. In your email please be as specific as possible so that JAMRS can ensure that the correct analysis is conducted. 
OVERVIEW REPORT



Appendix C 


\section{PAGE LEFT BLANK INTENTIONALLY}


NOVEMBER 2004 DOD YOUTH POLLING

\section{FALL 2004 [DRAFT] \\ EXPECTED FIELDING DATE 10/06/04}

\section{PROJECTED TIME: 20 minutes}

Objective:

Target Audience/Screening:

Target Field Dates:

Length:

Geography:

Sample Size:

Target:
The objective of this research is to conduct regular quantitative polling among the youth audience. Each poll will assess and track propensity, employment and education status. The poll will also be tailored to include questions on current events or topical areas of interest. Wirthlin Worldwide will conduct telephone interviews with youth two times per year -- in April and October.

Each household will be screened for youth who meet the following criteria:

- $\quad$ Are at least 16 years old, and less than 22 years old

- Have never served in the US Armed Forces and are not, at the time of the interview, accepted for such Service (Service includes the active and Reserve components of the US Army, Navy, Air Force, Marine Corps and Coast Guard).

- Are not enrolled in postsecondary reserve officer's training corps (ROTC) programs

If there is an individual in the household who meets the criteria but is away at college (living in a dormitory, fraternity house or student housing) will ask for the telephone number.

If there is more than one person in the household who meets those criteria, we will select the respondent in the household between the ages of 16 and 21 with the most recent birthday prior to the interview date. If that individual is away at college (living in a dormitory, fraternity house or temporary housing), we will ask for the telephone number and name of the youth and place that number in the callback queue. There will be no within household substitution of the designated respondent, even if the designated respondent does not qualify for the interview (e.g., is currently in the military, etc.).

Pre-test October 06, 2004

Launch study on October 09, 2004

This interview should last approximately 20 minutes.

$100 \%$ United States - including Alaska, Hawaii and the District of Columbia

$\mathrm{N}=3,300$ (approximately)

REGION: WirthlinWorldwide is now using a 9-point Geocode (see attached)

New England (5.06\%)

Connecticut, Maine, Massachusetts, New Hampshire, Rhode Island, Vermont

Mid-Atlantic (14.33\%) New Jersey, New York, Pennsylvania

South Atlantic (18.73\%) Delaware, Maryland, West Virginia, Virginia, North Carolina, South Carolina, Georgia, Florida, District of Columbia

East South Central (6.09\%) Mississippi, Alabama, Tennessee, Kentucky

East North Central (16.01\%)Illinois, Indiana, Michigan, Ohio, Wisconsin

West North Central (6.82\%) Iowa, Kansas, Missouri, Nebraska, North Dakota, South Dakota, Minnesota

West South Central (10.89\%) Texas, Louisiana, Arkansas, Oklahoma

Mountain (6.33\%) Arizona, Colorado, Idaho, Montana, Nevada, New Mexico, Utah, Wyoming

Pacific (15.75\%) California, Oregon, Washington, Hawaii and Alaska 
Sample:

Dialing Procedures:

Callback Procedures:

Refusal Conversion:

Pre-test:

Sample Mgt \& Replicates:
Random A sample with minimum of two working blocks. All sample will be screened for business numbers. Additionally, a stratified random sampling will be used. The exchanges will be stratified by concentration of the rare population, and oversample the strata with high concentrations. After classifying the exchanges into strata, the telephone numbers in the exchanges with the higher proportion of members will be sampled at a higher rate than the numbers in the other strata. This procedure is being used to improve the precision of estimates of African Americans and Hispanics.

Interviews will be conducted Monday through Thursday during the evening hours and Saturday and Sunday during the daytime and evening hours. No dialing will occur on Fridays. The fieldwork will take place from WirthlinWorldwide's telephone center located in Orem, Utah and at DIR's telephone center located in Los Angeles, CA. Both phone centers will utilize computer-assisted telephone interviewing (CATI).

Plan an initial call and maximum of nine callbacks. If a household is not reached after ten calls, we will substitute another randomly selected household. Callbacks will be scheduled on different days, different times of the day and in different weeks.

All initial refusals will be put into a queue to be worked by a group of interviewer specialists, trained and experienced in refusal conversion. Up to an additional three callbacks, conducted at different times and days, will be made. If a household is not reached after three calls or if a second refusal occurs, a "hard" refusal will be recorded on the final disposition. Experience shows that between $10 \%$ and $14 \%$ of the competed interviews will come from refusal conversions.

We will conduct a pretest of the survey instrument on October 06, 2004 in Orem, Utah telephone facility. We will conduct 30 interviews. If the pretest interviews go smoothly and no revisions are made to the questionnaire, they are included in the final data set. No more than 5 interviewers should work on the pre-test, this will ensure that the pre-test does not conclude too rapidly.

We will release sample in replicates. All replicates will be dialed until exhausted and then closed out. Once a replicate has been loaded, it must be dialed all the way through before the study can finish. A sample record is considered exhausted once it has obtained a final disposition. This means that the interviewers must continue to dial and conduct interviews even if 3,300 complete interviews have been completed - interviewers must dial through the entire replicate. To eliminate having too many extra completes, smaller replicates will be loaded toward the end of the interview cycle. NO NEW REPLICATE IS TO BE LOADED WITHOUT THE APPROVAL OF COURTNEY ZEGARSKI . Courtney can be reached during work hours at (703) 480- 1900 and during non-work hours at (202) 321-3913 (home/ cell). 
RESPONDENT INFLUENCERS AGED $\geq 22$ AND $\leq 85$

\section{GENERAL INSTRUCTIONS}

Target Audience:

Screening:

Field Dates:

Length:

Geography:

Sample Size:

Soft Quotas:
Each household will be screened for adults between the ages 22 and 85 who influence youth between the ages of 12 and 21 .

Each household will be screened for adults who meet the following criteria:

- Are at least 22 , but less than 85 years old

- Influencers of youth ages 12 to 21 .

- Includes coaches, clergy, scout leaders, employers, teachers, church lay people, volunteers, guidance counselors and mentors.

Pre-test October 6, 2004

Launch study on October 9, 2004

This interview should last approximately 20 minutes.

100\% United States - including Alaska, Hawaii and the District of Columbia

$\mathrm{N}=900$ adult influencers aged 22 to 85 (40\% incidence).

GENDER: $52 \%$ Female, $48 \%$ Male within each region

REGION: WirthlinWorldwide uses a 9-point Geocode

New England (5.06\%)

Mid-Atlantic (14.33\%)

South Atlantic (18.73\%)

East South Central (6.09\%)

East North Central (16.01\%)

West North Central (6.82\%)

West South Central (10.89\%)

Mountain (6.33\%)

Pacific $(15.75 \%)$
Connecticut, Maine, Massachusetts, New Hampshire, Rhode Island, Vermont

New Jersey, New York, Pennsylvania

Delaware, Maryland, West Virginia, Virginia, North Carolina, South Carolina, Georgia, Florida, District of Columbia

Mississippi, Alabama, Tennessee, Kentucky Illinois, Indiana, Michigan, Ohio, Wisconsin

Iowa, Kansas, Missouri, Nebraska, North Dakota, South Dakota, Minnesota

Texas, Louisiana, Arkansas, Oklahoma

Arizona, Colorado, Idaho, Montana, Nevada, New

Mexico, Utah, Wyoming

California, Oregon, Washington, Hawaii and Alaska
Sample:

Dialing Procedures:

Callback Procedures:

Pre-test:
Random A sample, with minimum of two working blocks. All samples will be screened for business numbers.

Interviews will be conducted during the evening and weekend hours. The fieldwork will take place from WirthlinWorldwide's telephone center located in Orem, Utah and at DIR's telephone center located in Los Angeles, CA. Both phone centers will utilize computer assisted telephone interviewing (CATI).

Plan an initial call and maximum of nine callbacks. If a household is not reached after ten calls, we will substitute another randomly selected household. Callbacks will be scheduled on different days, different times of the day and in different weeks.

We will conduct a pre-test of the survey instrument on October 6, 2004 in our Orem, Utah telephone facility. We will conduct thirty interviews. If the pretest interviews go smoothly and no revisions are made to the questionnaire, they will be included in the final data set. 
SCREENER AND INTRODUCTION

[NOTE TO INTERVIEWER: BE PREPARED FOR PARENTS TO ASK YOU (WHEN YOU ARE SCREENING OR DURING THE INTERVIEW) WHO YOU ARE AND WHAT YOU ARE ASKING THEIR KIDS. WE WILL HAVE A PRINTED SHEET WITH A SCRIPTED ANSWER - YOU SHOULD KEEP THIS AT YOUR STATION]

\section{SCRIPT IF PARENT WANTS TO KNOW MORE INFORMATION OR INTERRUPTS DURING THE INTERVIEW.}

My name is of Wirthlin Worldwide, a national independent research firm. I am calling for a study that is being conducted for the United States Government and am interested in speaking with your [son/daughter] about [his/her] opinions about being a young adult today and thoughts about potential careers. This study is very important, and results from it will be used by government officials, including congress, to develop important policy decisions. We are not trying to sell anything - we are only interested in [his/her] opinions. We also will hold [his/her] answers in the strictest of confidence - in no way will [he/she] ever be identified as a participant in this study. Furthermore, all information provided is protected under the Privacy Act of 1974. Would it be okay to talk to [him/her] about these issues?

\section{IF PARENT WANTS TO KNOW MORE:}

The survey contains questions about current education and employment status. There are questions dealing with their future plans - in particular after high school or college. The survey continues with questions related to the impressions that they have regarding various post-high school opportunities and ends with some basic demographic questions.

IF PARENT WANTS TO STAY ON THE PHONE WHILE THE SURVEY IS BEING CONDUCTED: I am more than happy to have you listen in on this interview, but I need to stress that the answers have to be directly from the designated respondent and not you. If you have questions along the way I will be more than happy to answer them, but please refrain from answering my questions for your child.

\section{IF THE PARENT WANTS TO CONTACT SOMEONE:}

If you have any questions about the questionnaire, the confidentiality issue, or about the validity of the study and the government's involvement, please email Courtney Zegarski of Wirthlin Worldwide, at czegarski@wirthlin.com. (If respondent does not have access to email give out phone number (703)4801900 . 
INTRO1 Hello, I'm of Wirthlin Worldwide, a national, independent research firm and I am calling for a study that is being conducted for the United States Government. We are interested in speaking with people between the ages of 16 and 21. Does your household include individuals between the ages of 16 and 21 who either live in the household or are away temporarily or living at school in a dormitory, fraternity or sorority house?
0. No
1. Yes
99. DK/REF

\section{IF INTRO1=1, ASK S11, ELSE GO TO INFLUENCER INTRO (INFINTRO1).}

S11. How many individuals are there in your household between the ages of 16 and 21 who either live in the household or are away temporarily or living at school in a dormitory, fraternity or sorority house?

RECORD ANSWER

99. DK/REF [THANK AND TERMINATE]

\section{\{TALLY QUOTA ‘B1’AT THIS POINT\}}

\section{IF S11 = 0, GO TO INFLUENCER INTRO (INFINTRO1)}

\section{IF S11 > 0, ASK GPA}

GPA. We are conducting this study to find out the opinions and career paths of young adults and we would like to have the responses of the person between the ages of 16 and 21 who has had the most recent birthday. Could I please speak with that person? [INTERVIEWER: IF THE ANSWER IS NO, CLARIFY WHY]

1. Yes

2. No, respondent isn't available but resides in the household (i.e., not home)

3. No, respondent isn't available because they are temporarily away or living at school in a dormitory, fraternity or sorority house

4. No, respondent won't allow you to talk with them

\section{IF GPA=1, WAIT UNTIL RESPONDENT GETS ON THE PHONE AND READ PRIV1.}

IF GPA $=2$, ARRANGE CALLBACK

IF GPA=3, ASK S8

IF GPA=4, THANK AND TERMINATE

S8. We are conducting this study to find out the opinions and career paths of young adults and we would like to have the responses of the person who is away. Could I please have his/her first name and telephone number with area code?

0 . No

1. Yes

99.DK/REF

IF S8=1, RECORD NAME AND NUMBER AND THEN THANK. PLACE NEW NAME AND NUMBER IN CALLBACK QUEUE. 


\section{WHEN RESPONDENT BETWEEN THE AGES OF 16 AND 21 WITH THE MOST RECENT BIRTHDAY IS ON THE PHONE, READ PRIV1}

PRIV1. Hello, I'm of Wirthlin Worldwide, a national, independent research firm. We are conducting a study to find out more about the opinions and career plans of young adults. The study is being conducted for the Department of Defense. Results of this study will be used in reports to Congress, and in the development of important policy decisions. For quality purposes, my supervisor may monitor this call. (DO NOT PAUSE)

All information you provide is protected under the Privacy Act of 1974. Your identity will not be released for any reason and your participation is voluntary. You are entitled to a copy of the Privacy Act Statement. Would you like a copy of this statement?

0 . No

1. Yes, record mailing address

99.DK/REF

S2. Just to confirm, what is your gender? [IF RESPONDENT REFUSES, ENTER GENDER BY OBSERVATION]

1. Male

2. Female

\section{[ASK EVERYONE]}

S10. Are you a United States Citizen?

0 . No

1. Yes

99.DK/REF

S1. What is your date of birth? [ENTER IN SIX DIGIT FORMAT MM/DD/YY]

RECORD MONTH/DAY/YEAR

99. DK/REF

\section{IF AGE IS NOT BETWEEN 16-21 VERIFY BIRTH DATE ASK GPA IF AGE IS BETWEEN 16 AND 21, ASK DEM2C}

DEM2C. Have you ever been in the military, or are you in a delayed entry program (DEP), college ROTC, or one of the service academies? [MILITARY SERVICE INCLUDES ALL BRANCHES (FULLTIME OR AS RESERVIST, NATIONAL GUARD), SERVICE ACADEMIES OR COLLEGE (NOT H.S.) ROTC. ALSO ENTER 'YES' IF ACCEPTED INTO SERVICE AND WAITING TO BEGIN.]

0. No

1. Yes

99.DK/REF 
IF DEM2C=0, ASK DEM10, ELSE THANK AND TERMINATE

DEM10. Do you consider yourself to be of Hispanic, Latino or Spanish origin?

0. No

1.Yes, Mexican, Mexican American, Chicano, Puerto Rican, Cuban, or other Spanish/Hispanic/Latino origin.

99. DK/REF

DEM11 I'm going to read a list of racial categories. Please select one or more to describe your race. Are you...[READ PUNCHES 1-5.] [NOTE: IF RESPONDENT SAYS 'DON'T KNOW” OR DOESN'T MENTION A PUNCH BELOW, SAY: "WHICH OF THE FOLLOWING RACE CATEGORIES DO YOU MOST CLOSELY IDENTIFY WITH?”] [CODE UP TO 5 RESPONSES]

1. White

2. Black or African-American

3. American Indian or Alaskan Native

4. Asian (e.g., Asian Indian, Chinese, Filipino, Japanese, Korean, Vietnamese)

5. Native Hawaiian or Other Pacific Islander (e.g., Samoan, Guamanian or Chamorro)

6. [DO NOT READ] Other HISPANIC ONLY (Mexican, Mexican American, Chicano, Puerto Rican, Cuban, or other Spanish/Hispanic/Latino origin.)

99. DK/REF [THANK AND TERMINATE]

[IF DEM11=6 ONLY, ASK DEM11A]

DEM11A. In addition to being Hispanic, do you consider yourself to be [READ PUNCHES 1-5] [CODE UP TO 5 RESPONSES]

1. White

2. Black or African-American

3. American Indian or Alaskan Native

4. Asian (e.g., Asian Indian, Chinese, Filipino, Japanese, Korean, Vietnamese)

5. Native Hawaiian or Other Pacific Islander (e.g., Samoan, Guamanian or Chamorro)

6. Not Applicable

99. DK/REF

\section{EDUCATION}

\section{[RESPONDENTS INCLUDE NON-CITIZENS]}

EDU1. I'd like to ask you about your schooling. Are you currently enrolled in school or a training program?

0. No

1. Yes

99. DK/REF 
IF EDU1=1, ASK EDU2 [IF RESPONDENT IS CURRENTLY ENROLLED IN SCHOOL]

EDU2. What grade or year of school are you in? [DO NOT READ, ACCEPT SINGLE RESPONSE] [IF RESPONDENT ANSWERS IN A GENERAL SENSE, FOR INSTANCE “COLLEGE” MAKE SURE YOU CLARIFY WHICH TYPE OF COLLEGE AND WHICH YEAR]

1. Less than 8th Grade

2. 8th Grade

3. 9th Grade - High School

4. 10th Grade - High School

5. 11th Grade - High School

6. 12th Grade - High School

7. 1st Year College or University (Freshman)

8. 2nd Year College or University (Sophomore)

9. 3rd Year College or University (Junior)

10. 4th Year College or University (Senior)

11. 5th Year College or University

12. 1st Year Graduate or Professional School

13. 2nd Year Graduate or Professional School (MA/MS)

14. 3rd Year Graduate or Professional School

15. More than 3 Years Graduate or Professional (Ph.D.)

16. 1st Year Junior or Community College

17. 2nd Year Junior or Community College (AA/AS)

18. 1st Year Vocational, Business or Trade School

19. 2nd Year Vocational, Business or Trade School

20. More than 2 Years Vocational, Business or Trade School

99. DK/REF

IF EDU1=0 or 99, ASK EDU3 [IF RESPONDENT IS NOT CURRENTLY ENROLLED IN SCHOOL]

EDU3. What is the highest grade you have completed and received credit for? [IF RESPONDENT ANSWERS IN A GENERAL SENSE, FOR INSTANCE "I GRADUATED FROM COLLEGE" MAKE SURE YOU CLARIFY HOW MANY YEARS THEY WERE THERE AND WHAT TYPE OF COLLEGE THEY ATTENDED - FOUR YEAR, TWO YEAR, GRADUATE, ETC.]

1. Less than 8th Grade

2. 8th Grade

3. 9th Grade - High School

4. 10th Grade - High School

5. 11th Grade - High School

6. 12th Grade - High School

7. 1st Year College or University (Freshman)

8. 2nd Year College or University (Sophomore)

9. 3rd Year College or University (Junior)

10. 4th Year College or University (Senior)

11. 5th Year College or University

12. 1st Year Graduate or Professional School

13. 2nd Year Graduate or Professional School (MA/MS)

14. 3rd Year Graduate or Professional School

15. More than 3 Years Graduate or Professional (Ph.D.)

16. 1st Year Junior or Community College

17. 2nd Year Junior or Community College (AA/AS) 
18. 1st Year Vocational, Business or Trade School

19. 2nd Year Vocational, Business or Trade School

20. More than 2 Years Vocational, Business or Trade School

99. DK/REF

\section{ASK IF EDU2 = 3-20 OR EDU3=3-20}

EDU5. What grades do you or did you usually get in high school? [READ RESPONSE CATEGORIES

1-7]. [IF RESPONDENT NEEDS CLARIFICATION, READ THEM THE NUMERICAL AVERAGES, OTHERWISE JUST READ THE LETTER GRADES]

1. Mostly A's (Numerical average of 90-100)

2. Mostly A's and B's (85-89)

3. Mostly B's (80-84)

4. Mostly B's and C's (75-79)

5. Mostly C's (70-74)

6. Mostly C's and D's (65-69)

7. Mostly D's and lower (64 and below)

8. Never in high school

99. DK/REF

\section{DEMOGRAPHIC - EMPLOYMENT STATUS}

EMP1. Now, I'd like to ask you about your employment status. Are you currently employed either fullor part-time?

0 . No

1. Yes

99. DK/REF

IF QEMP1=1 THEN ASK QEMP2 [IF RESPONDENT IS CURRENTLY EMPLOYED]

EMP2. How many hours per week in total do you work at your job?

RECORD RESPONSE

99. DK/REF

EMP5. How difficult is it for someone your age to get a full-time job in your community? Is it...[READ 1-4]

1. Almost Impossible

2. Very Difficult

3. Somewhat Difficult

4. Not Difficult at All

99. DK/REF 
FUTURE PLANS AND PROPENSITY

FPP1. Next, I'd like to ask you about your plans for the future. What do you think you might be doing [INSERT BASED ON RESPONSE TO EDU1 [CURRENTLY ENROLLED IN SCHOOL OR TRAINING PROGRAM] AND EDU2 [WHAT GRADE OR YEAR OF SCHOOL ARE YOU IN] AS FOLLOWS: [DO NOT READ LIST] [ACCEPT MULTIPLE RESPONSES] [PROBE UNTIL UNPRODUCTIVE] [PUNCH 7, 8 \& 99 MUST BE SINGLE PUNCH]

IF EDU2 = 3, 4, 5 OR 6 [RESPONDENT IS CURRENTLY ENROLLED IN SCHOOL AND IS IN HIGH SCHOOL] INSERT “once you finish high school?"

IF EDU2 $=7,8,9,10,11,12,13,14,15,16,17,18,19$ OR 20 [RESPONDENT IS CURRENTLY ENROLLED IN SCHOOL AND IS IN COLLEGE, GRADUATE, JUNIOR/COMMUNITY OR VOCATIONAL SCHOOL] INSERT "once you finish college?"

IF EDU2 = 1 OR 2 OR IF EDU1 = 0 OR 99 [RESPONDENT IS NOT CURRENTLY ENROLLED IN SCHOOL OR IS IN $\boldsymbol{8}^{\text {TH }}$ GRADE OR LESS] INSERT “in the next few years?" [WHEN PROBING EMPHASIZE “IN THE NEXT FEW YEARS”]

1. Going to school full-time

2. Going to school part-time

3. Working full-time

4. Working part-time

5. Joining the Military/Service

6. Staying at Home

7. Doing nothing

8. Undecided / Have not decided yet

9. Community Service

10. Other, Specify

99. DK/REF

IF FPP1=5 ASK FPP2 [IF RESPONDENT SAYS THEY ARE GOING TO MILITARY]

FPP2. You said you might be joining the military. Which branch of the service would that be? [DO NOT READ ANSWER CATEGORIES - FIT RESPONSE TO PRE-CODED ANSWERS.] [IF RESPONDENT MENTIONS MORE THAN ONE BRANCH,: Which branch are you most likely to join?

IF RESPONDENT MENTIONS NATIONAL GUARD, CLARIFY WHETHER THAT IS ARMY NATIONAL GUARD OR AIR NATIONAL GUARD IF ARMY NATIONAL GUARD, CODE AS ARMY, IF AIR NATIONAL GUARD, CODE AS AIR FORCE.

IF RESPONDENT MENTIONS THUNDERBIRD OR STEALTH FORCE, CODE AS AIR FORCE. IF THEY MENTION GOLDEN KNIGHTS OR GREEN BERET, CODE AS ARMY.

IF THEY MENTION SAILORS, SEALS, BLUE ANGELS OR SUBMARINERS, CODE AS NAVY.]

1. Air Force

2. Army

3. Coast Guard

4. Marine Corps

5. Navy

99. DK/REF 
IF FPP2 = 1 OR 2 [IF RESPONDENT SAYS THEY ARE INTERESTED IN JOINING THE AIR FORCE OR ARMY] FPP3A. Which type of service would that be? Would it be... [READ 1-3]?
1. Active Duty
2. The Reserves
3. The National Guard
99. DK/REF

IF FPP2 = 3, 4 OR 5 [IF RESPONDENT SAYS THEY ARE INTERESTED IN JOINING THE COAST GUARD, MARINE CORPS OR NAVY]

FPP3B. Which type of service would that be? Would it be... [READ 1-2]?
1. Active Duty
2. The Reserves
99. DK/REF

\section{IF FPP1=3 OR 4 ASK FPP4 [IF RESPONDENT SAYS THEY MIGHT BE WORKING]}

FPP4. You said you might be working. What type of job would you have? Would it be a temporary job while you finish school or training, any job you can get to support yourself, or a job that could begin a long-term career?

1. Temporary job while you finish school or training

2. Any job you can get to support yourself

3. Job that could begin a long-term career

99. DK/REF

IF FPP1=1 OR 2 ASK FPP5 [IF RESPONDENT SAYS THEY ARE GOING TO SCHOOL]

FPP5. What kind of school or college would you like to attend? [READ 1-5]

$1 \quad$ High School

2 Vocational, Business or Trade School

3 2-Year Junior or Community College

4 4-Year College or University

5 Graduate or Professional School

99 DK/REF 


\section{[ASK EVERYONE]}

FPP8. What is the highest grade or year of school or college that you would eventually like to complete? [If Respondent answers in a general sense, such as "finish college" then clarify TYPE and YEAR of school.] [DO NOT READ LIST]



FPP9. Now, I'd like to ask you how likely it is that you will be serving in the military in the next few years? Would you say...[ROTATE TOP TO BOTTOM, BOTTOM TO TOP AND READ 1-4]

$1 \quad$ Definitely

2 Probably

3 Probably Not

4 Definitely Not

99 DK/REF 
FPP10. How likely is it that you will be serving on active duty in the [RANDOMIZE AND READ A-E]? Would you say... [ROTATE TOP TO BOTTOM, BOTTOM TO TOP AND READ 1-4]?
A Coast Guard
B Army
C Air Force
D Marine Corps
E Navy

$1 \quad$ Definitely

2 Probably

3 Probably Not

4 Definitely Not

99 DK/REF

NOTE TO CATI TECH: ROTATE FIRST/SECOND FPP11/11A AND FPP12/12A

FPP11. How likely is it that you will be serving in the National Guard? [ROTATE TOP TO BOTTOM, BOTTOM TO TOP AND READ 1-4]

$\begin{array}{ll}1 & \text { Definitely } \\ 2 & \text { Probably } \\ 3 & \text { Probably Not } \\ 4 & \text { Definitely Not } \\ 99 & \text { DK/REF }\end{array}$

IF FPP11 = 1 OR 2, ASK FPP11A

FPP11A. Would that be the... [RANDOMIZE AND READ 1-2]?

$1 \quad$ Air National Guard

2 Army National Guard

99 DK/REF

FPP12. How likely is it that you will be serving in the Reserves? [ROTATE TOP TO BOTTOM, BOTTOM TO TOP AND READ 1-4]

$1 \quad$ Definitely

2 Probably

3 Probably Not

4 Definitely Not

99 DK/REF 
IF FPP12 = 1 OR 2, ASK FPP12A

FPP12A. Would that be the... [RANDOMIZE AND READ 1-5]?

1 Air Force Reserve

2 The Army Reserve

3 The Coast Guard Reserve

4 The Marine Corps Reserve

5 The Naval Reserve

99 DK/REF

IF TWO OR MORE OF ANY ACTIVE, RESERVE, GUARD SERVICES ARE ANSWERED "DEFINITELY" OR "PROBABLY” IN QUESTIONS FPP10, FPP11 OR FPP12, ASK FPP14

FPP14. You mentioned you might serve in more than one military service. Which service are you most likely to serve in? [DO NOT READ ANSWER CATEGORIES, FIT RESPONSE TO PRECODE - ACCEPT SINGLE RESPONSE] [INTERVIEWER NOTE: IF ANSWER IS GENERAL, PLEASE CLARIFY IF ACTIVE DUTY, RESERVES OR GUARD.]

$\begin{array}{ll}1 & \text { Air Force } \\ 2 & \text { Army } \\ 3 & \text { Coast Guard } \\ 4 & \text { Marine Corps } \\ 5 & \text { Navy } \\ 6 & \text { Air National Guard } \\ 7 & \text { Army National Guard } \\ 8 & \text { Air Force Reserve } \\ 9 & \text { Army Reserve } \\ 10 & \text { Coast Guard Reserve } \\ 11 & \text { Marine Corps Reserve } \\ 12 & \text { Naval Reserve } \\ 99 & \text { DK/REF }\end{array}$

[ASK ALL]

FPP15. Before we talked today, had you ever considered the possibility of joining the military? Would you say you...[ROTATE TOP TO BOTTOM, BOTTOM TO TOP AND READ ANSWERS 1-3]

$1 \quad$ Never Thought About It

2 Gave It Some Consideration

3 Gave It Serious Consideration

99 DK/REF

ASK IF QFPP9=1,2 or 3 OR QFPP10=1,2 or 3 OR IF FPP11=1, 2, or 3 OR FPP12=1, 2, or 3

ADVNEWA. When thinking about military service, when would you be more likely to consider the military as an option... [READ LIST]

1. Immediately after high-school, as a post-high school graduation option, or

2. A few years after high-school, possibly after a few years of college or vocational training

99. DK/ REF 


\section{FAVORABILITY}

FAV1. Using all that you know or have heard about the US military, please rate the US military using a 10 point scale where 1 means VERY UNFAVORABLE and 10 means VERY FAVORABLE. How would you rate the US Military?

\section{RECORD RATING}

99 DK/REF

FAV2. Using all that you know or have heard about the various branches of the US military, please rate each branch using a 10 point scale where 1 means VERY UNFAVORABLE and 10 means VERY FAVORABLE. How would you rate the [RANDOMIZE AND READ A-E]?

RECORD RATING

99 DK/REF
A. Air Force
B. Army
C. Coast Guard
D. Marine Corps
E. Navy

FAV3. Now, using all that you know or have heard, please rate the US National Guard and Reserves using a 10 point scale where 1 means VERY UNFAVORABLE and 10 means VERY FAVORABLE. How would you rate the [RANDOMIZE AND READ A-B]?

RECORD RATING

$99 \mathrm{DK} / \mathrm{REF}$

1. Reserves

2. National Guard

\section{KNOWLEDGE OF MILITARY}

KW2. Let's talk about your knowledge of the U.S. military. Please use a scale from 1 to 10 where 1 means NOT AT ALL KNOWLEDGEABLE and 10 means EXTREMELY

KNOWLEDGEABLE. Please tell me how knowledgeable you are about the U.S. Military.

RECORD ANSWER

99. DK/REF 
ADV4. Sometime during the past 12 months have you spoken with either of your parents about what you are going to do with your future?
0. No
1. Yes
99. DK/REF

[IF ADV4 = 1 ASK ADV10]

ADV10. Did you initiate that conversation?
0. No
1. Yes
99. DK/REF

[IF ADV4 = 1, ASK ADV8]

ADV8. In this discussion, did you discuss the possibility of you joining the military with them?
0. No
1. Yes
99. DK/REF

[IF ADV8 = 1, ASK ADV12]

ADV12. When discussing the military, were they... [READ LIST AND ROTATE TOP TO BOTTOM, BOTTOM TO TOP]?
1. Completely Positive
2. Mostly Positive
3. Neutral
4. Mostly Negative
5. Completely Negative
99. DK/REF

\section{ASK ALL}

ADV4A. Sometime during the past 12 months have you spoken with one of your teachers, guidance counselors or coaches about what you are going to do with your future?

0 . No

1. Yes

98. Not Applicable

99. DK/REF 
[IF ADV4A = 1 ASK ADV10A]

ADV10A. Did you initiate that conversation?
0. No
1. Yes
99. DK/REF

[IF ADV4A = 1, ASK ADV8A]

ADV8A. Did you discuss the possibility of you joining the military with them?
0 . No
1. Yes
99. DK/REF

[IF ADV8A = 1, ASK ADV12A]

ADV12A. When discussing the military, were they... [READ LIST AND ROTATE TOP TO BOTTOM, BOTTOM TO TOP]?
1. Completely Positive
2. Mostly Positive
3. Neutral
4. Mostly Negative
5. Completely Negative
99. DK/REF 
REC1. I now would like to ask you a few questions regarding military recruiters. First, have you ever spoken with a military recruiter? [1 QP]

0. No

1. Yes

99. DK/REF

REC2. Now I am going to read you a couple of statements. After each one, please tell me if you agree or disagree with that statement. The [FIRST/NEXT] is [ROTATE AND READ LIST A-E]. Do you [ROTATE BOTTOM TO TOP, TOP TO BOTTOM AND READ RESPONSE OPTIONS]?

A. Military recruiters usually present a truthful picture of military service

B. The information that military recruiters provide is trustworthy

C. People entering the military often don't get the benefits promised by military recruiters

D. Military recruiters care about the well-being of people interested in military service

E. Military recruiters use "high pressure" sales tactics to get people to join the military

1. Completely agree

2. Mostly agree

3. Neither agree nor disagree

4. Mostly disagree

5. Completely disagree

99. DK/REF 
REC3. If a military recruiter called your home to speak with you, how likely would you be to do each of the following? Please use a scale where 1 means not at all likely and 5 means very likely. The [FIRST/NEXT] is [RANDOMIZE AND READ A AND C] ...

A. Speak with the military recruiter

B. Have a conversation with your parents about the military

1. Not at all likely

2.

3.

4.

5. Very likely

99. DK/REF

REC4. If you received military recruiting materials in the mail, how likely would you be to do each of the following? Please use a scale where 1 means not at all likely and 5 means very likely. The [FIRST/NEXT] is [RANDOMIZE AND READ A-D]...

A. Throw the mail away without looking at it

B. Read the information

C. Submit request to sender asking them to stop sending recruiting materials to you

D. Have a conversation with your parents about the military

1. Not at all likely

2.

3.

4.

5. Very Likely

99. DK/REF 


\section{MEDIA SOURCES}

MEDNEW. Assume that you wanted to get information about the military and what it has to offer its members. I am going to read you a list of places that you can get information about the military. Using a scale of 1 to 5 where 1 means NOT AT ALL LIKELY and 5 means VERY LIKELY, I would like you to tell me how likely you would be to use each source if you were looking for information about the military. The [FIRST/NEXT] is [RANDOMIZE AND READ A-U]. INTERVIEWER INSTRUCTION: REREAD SCALE EVERY 5 ITEMS.

SPLIT SAMPLE : 1/2 SAMPLE SHOULD RECEIVE A-I AND 1/2 SAMPLE SHOULD RECEIVE JR. EVERYONE SHOULD GET S,T AND U. NOTICE REORDERING OF SOURCES
A. Family members
B. Teachers, guidance counselors and coaches
C. Advertisements in the newspaper
D. Information you receive in the mail
E. TV News
F. Radio Commercials
G. Magazine Articles
H. Fiction Books
I. The Internet
J. Friends
K. Military Recruiters
L. Newspaper Articles
M. TV Commercials
N. TV entertainment programs
O. Radio Programs
P. Advertisements in magazines
Q. Non-fiction Books
R. Movies
S. Military Websites
T. Military Advertisements (print, radio, TV)

1. Not at all likely

2.

3.

4.

5. Very likely

99. DK/REF 
MED5. Now I am going to read you the same list of places that you can get information about the military from. This time I want you to use a scale of 1 to 5 where 1 means DON'T TRUST AT ALL and 5 means TRUST COMPLETELY, I would like you to tell me how much you trust the information that you get from each person or place if you were looking for information about the military. The [FIRST/NEXT] is [RANDOMIZE AND READ A-U].

INTERVIEWER INSTRUCTION: REREAD SCALE EVERY 5 ITEMS.

SPLIT SAMPLE : 1/2 SAMPLE SHOULD RECEIVE A-I AND 1/2 SAMPLE SHOULD RECEIVE JR. EVERYONE SHOULD GET S,T AND U. SHOULD RECEIVE SAME LIST THAT THEY GOT IN MEDNEW. NOTICE REORDERING OF SOURCES
A. Family members
B. Teachers, guidance counselors and coaches
C. Advertisements in the newspaper
D. Information you receive in the mail
E. TV News
F. Radio Commercials
G. Magazine Articles
H. Fiction Books
I. The Internet
J. Friends
K. Military Recruiters
L. Newspaper Articles
M. TV Commercials
N. TV entertainment programs
O. Radio Programs
P. Advertisements in magazines
Q. Non-fiction Books
R. Movies
S. Military Websites
T. Military Advertisements (print, radio, TV)
1. Don't Trust at all
2.
3.
4.
5. Trust Completely
99. DK/REF 


\section{ECONOMIC INDICATORS}

IND2. Are individuals more likely to have a good paying job in the military, in a civilian job or equally in both?
1. Military
2. Civilian job
3. Equally in both
99. DK/REF

IND3. Four years from now, do you think the economy will be better than, worse than, or about the same as it is today?
1. Better
2. Worse
3. About the Same
99. DK/REF

RD/WT. Generally speaking, would you say that things in the COUNTRY are going in the RIGHT DIRECTION, or have they pretty seriously gotten off on the WRONG TRACK?

[INTERVIEWERS: DO NOT ACCEPT “NO OPINION” OR “DK/REF” AS A FIRST ANSWER. PROBE AT LEAST ONCE SAYING: “WHAT’S YOUR FIRST IMPRESSION?”]

1. Right Direction

2. Wrong Track

3. (DO NOT READ) No Opinion

99. (DO NOT READ) DK/Refused

\section{CURRENT EVENTS}

CUR7. Do you support or oppose U.S. Military troops being in Iraq?

1. Support Troops

2. Oppose Troops

3. Neither [Do Not Read]

99. DK/REF

CUR9. Does the current situation with the war on terrorism make you more likely or does it make you less likely to join the military?

1. More likely

2. Doesn't change the likelihood (DO NOT READ)

3. Less likely

99. DK/REF 
CUR10. Do you approve or disapprove of the way the Bush administration is -- [RANDOM ORDER].

Would that be strongly (approve/disapprove) or just somewhat (approve/disapprove)?
A. Handling Foreign Affairs
B. Using the U.S. Military Forces
1. Strongly Approve
2. Somewhat Approve
3. No opinion (DO NOT READ)
4. Somewhat Disapprove
5. Strongly Disapprove
99. DK/REF

Now I want you to think about the Federal government overall and the jobs it has available.

ADV14. Once they finish school, some people go to work for the Federal Government. I am going to read you a list and I would like to know how you think working for the U.S. Federal Government compares with other places you could work in regards to each item I read to you. The [FIRST/NEXT] is [RANDOMIZE AND READ A - L]. Does the U.S. Federal Government have 'BETTER', 'ABOUT THE SAME' OR WORSE' [READ AND ROTATE] [RANDOMIZE AND READ A-L] than most other places you could work?
A. Management
B. Pay
C. Health care benefits
D. Promotion opportunities
E. Work hours
F. Work environment
G. Job Security
H. Job Opportunities
I. Employees
J. Opportunities for minorities
K. Retirement Benefits
L. Reputation

1. Better than most

2. About the same as most

3. Worse than most

99. DK/REF 


\section{DEMOGRAPHICS}

THE LAST SET OF QUESTIONS ASK FOR SOME BACKGROUND INFORMATION ABOUT YOURSELF

DEM3. Please tell me whether you are currently...[READ LIST] [NOTE TO INTERVIEWER: IF RESPONDENT SAYS THEY ARE DATING, IN A RELATIONSHIP WITH A SIGNIFICANT OTHER, HAVE A BOY/GIRLFRIEND - YOU MUST CODE THEM AS SINGLE]

1. Single and have never been married

2. Widowed

3. Separated

4. Divorced

5. Married

6. Something else, specify

99. DK/REF

DEM20. Has your [INSERT A-I] ever served in the U.S. military?
A. Father
B. Mother
C. Brother
D. Sister
E. Uncle
F. Aunt
G. Grandparent
H. Cousin
I. Spouse $\{$ NOT ASKED IF DEM3 $=1\}$
0. No
1. Yes
99. DK/REF

DEM100. Are there any telephone numbers in your household in addition to... [INSERT TELEPHONE NUMBER]

0 . No

1. Yes

99. DK/REF

\section{ASK DEM101 IF DEM100=1}

DEM101. Is this/ Are these numbers for... [READ LIST]

1. Home use

2. Business and home use

3. Business use only

99. DK/ REF 


\section{ASK DEM12 IF QPRIV1=2 or 99}

DEM12. For research purposes only, please tell me your street address and zip code? Do you know your ZIP plus four? [9-digit ZIP code is preferred]

[RECORD STREET ADDRESS]

[RECORD ZIP CODE]

\section{ASK DEM13 IF QPRIV1=1}

DEM13. So that we may send you the copy of the Privacy Act of 1974 and for research purposes please tell me your address.

[RECORD STREET ADDRESS]

[RECORD CITY]

[RECORD STATE]

[RECORD ZIP CODE]

99. DK/REF

DEM17. Finally, I would like to ask you for your first and last name. Defense Department social scientists match youth's names to enlistment data to find out how the plans and opinions of American youth relate to enlistment rates. Your name, along with any other information you have provided, is protected under the Privacy Act of 1974. [NOTE TO INTERVIEWER: THIS IS VOLUNTARY INFORMATION SO IF THE RESPONDENT DOES NOT WANT TO PROVIDE THEIR FULL NAME, MOVE ONTO THE NEXT QUESTION] $\{$ ALLOW DK/REF

DEM14. FIPS CODE

DEM15. ZIP CODE [FROM SAMPLE]

\section{\{PROGRAMMER: DUMMY OUT DEM16\}}

DEM16. May I please have your name in case my supervisor needs to verify that this interview actually took place?

PRNT1. [NEED TO ADD PUNCH FOR INTERVIEWER TO INDICATE WHETHER OR NOT RESPONDENT'S PARENT WAS ON THE PHONE.]

INTERVIEWER: WAS THE RESPONDENT'S PARENT ON THE PHONE?

0 . No

1. Yes

99. DK/REF

Thank you very much for your time. 


\section{PAGE LEFT BLANK INTENTIONALLY}


